\title{
Estimation of 1945 to 1957 Food Consumption
}

Hanford Environmental

D. M. Anderson

D. J. Bates

T. L. Marsh

March 1993

Prepared for review and approval by the Technical Steering Panel and the Centers for Disease Control under Contract 200-92-0503(CDC)/18620(BNW)

Battelle, Pacific Northwest Laboratories

Richland, Washington 99352 


\section{LEGAL NOTICE}

This report was prepared by Battelle as an account of sponsored research activities. Neither Sponsor nor Battelle nor any person acting on behalf of either:

MAKES ANY WARRANTY OR REPRESENTTATION, EXPRESS OR IMPLIED, with respect to the accuracy, completeness, or usefulness of the information contained in this report, or that the use of any information, apparatus, process, or composition disclosed in this report may not infringe privately owned rights; or

Assumes any liabilities with respect to the use of, or damages resulting from the use of, any information, apparatus, process, or composition disclosed in this report.

Reference herein to any specific commercial product, process, or service by trade name, trademark, manufacturer, or otherwise, does not necessarily constitute or imply endorsement, recommendation, or favoring by Sponsor or Battelle.

Printed in the United States of America

Available to DOE and DOE contractors from the

Office of Scientific and Technical Information, P.O. Box 62, Oak Ridge, TN 37831. prices available from (615) 576-8401. FTS 626-8401.

Available to the public from the National Technical Information Service, U.S. Department of Commerce, 5285 Port Royal Rd., Springfield, VA 22161. 


\section{Preface}

In 1987, the U.S. Department of Energy (DOE) directed the Pacific Northwest Laboratory, which is operated by Battelle Memorial Institute, to conduct the Hanford Environmental Dose Reconstruction (HEDR) Project. The DOE directive to begin project work followed a recommendation by the Hanford Health Effects Review (HHER) Panel in 1986. The HHER Panel was formed to consider the potential health implications of past Hanford-Site releases of radioactive materials.

A December 1990 Memorandum of Understanding between the Sectetaries of the DOE and the U.S. Department of Health and Human Services (DHHS) transferred responsibility for managing the DOE's dose reconstruction and exposure assessment studies to the DHHS. This transfer resulted in the current contract between Battelle, Pacific Northwest Laboratories and the Centers for Disease Control and Prevention, an agency of the DHHS, to continue the project.

An 18-member Technical Steering Panel (TSP) was selected by the Vice Presidents for Research at major universities in Washington and Oregon to direct the project work. The TSP consists of experts in the various technical fields relevant to HEDR Project work and representatives from the states of Washington, Oregon, and Idaho; Native American Tribes; and the public.

The purpose of the HEDR Project is to estimate the radiation dose that individuals could have received as a result of emissions since 1944 from DOE's Hanford Site near Richland, Washington. The HEDR Project work is conducted under several technical and administrative tasks, among which is the Demography, Food Consumption, and Agriculture Task. The staff on this task provide the demographic, food consumption, food production, and distribution information necessary to estimate doses from radiation. That information is necessary because food is one pathway by which individuals may have ingested iodine-131, the largest contributor to the historical radiation dose from Hanford (Napier 1992a). To develop such information, sources and quantities of food and water consumed by individuals must be estimated. In particular, milk, eggs, and leafy vegetables represent potentially significant food pathways for iodine-131 (Napier 1992b). Therefore, the food consumption habits of the population in the HEDR study area are being examined to estimate the likely ingestion of potentially contaminated foods that contribute significantly to radiation dose as defined by the TSP dose decision level (Shleien 1992).

A previous study, Estimation of Food Consumption (Callaway 1992), estimated the 1945 and 1965 food-consumption habits of those people living in the 10 counties nearest to the Hanford Site. The purpose of that initial study was to demonstrate the feasibility of such a project. This study builds on the findings of the initial study with improved detail and reliability. The primary purpose of this study is to provide food consumption data to be used in dose calculations. This report fulfills HEDR Project Milestone 0602C. 


\section{Summary}

\section{Scope of Work}

This report details the methods used and the results of the study on the estimated historic levels of food consumption by individuals in the Hanford Environmental Dose Reconstruction (HEDR) study area from 1945-1957. This period includes the time of highest releases from Hanford and is the period for which data are being collected in the Hanford Thyroid Disease Study. These estimates provide the food-consumption inputs for the HEDR database of individual diets. This database will be an input file in the Hanford Environmental Dose Reconstruction Integrated Code (HEDRIC) computer model that will be used to calculate the radiation dose.

The report focuses on fresh milk, eggs, lettuce, and spinach. These foods were chosen because they have been found to be significant contributors to radiation dose based on the Technical Steering Panel dose decision level (Shleien 1992).

\section{Technical Approach}

The technicial approach used to estimate the historical levels of food consumption was presented in Anderson (1992) and reviewed by the TSP. The 1977-1978 Nationwide Food Consumption Survey (NFCS) (USDA 1983) of individual food intake was used to perform the analysis. The 1977-1978 data were used because the individual intake data that exist for 1945-1957 do not provide the necessary detail. The 3,735 coded food types of the NFCS were collapsed into 65 recognizable food types. This aggregation still allows enough variation in food types to adequately discern specific diet characteristics, and it allows historic food-consumption trends to be represented. These 65 food types will be further collapsed into the 9 groups used by the computer dose model.

Estimates of consumption for each type of food were derived for specific age/sex and urban/rural groups according to season. These estimates were converted (backcasted) from the 1977-1978 period to the years of interest (1945-1954) using a set of estimated conversion factors derived from national per capita food-disappearance (retail sales quantities) data maintained by the U. S. Department of Agriculture (USDA 1965, 1981). The report provides the full analytical methodology used to project consumption estimates back to the years in question.

This report also describes the aggregation of food groups to the level required for input to the dose model. The intent in this report is to show consumption estimates for specific foods that are dose relevant. 


\section{Results}

The data used in this analysis will be compiled into a database to be used as an input file to the HEDRIC dose model. This input file provides the dose model with the actual observations from the 1977-1978 NFCS and the backcasting ratios necessary to convert those estimates to any year in the 1945-1957 time span. These observations will be used within the dose model to reconstruct a potential sequence of daily diets over time to be used in reconstructing a person's potential ingestion pathways (Snyder et al. 1992). The diet data will be used as reference for individual dose calculations and will not be used when data are available for specific individuals. 


\section{Contents}

Preface $\ldots \ldots \ldots \ldots \ldots \ldots \ldots \ldots \ldots \ldots \ldots \ldots \ldots \ldots \ldots \ldots \ldots \ldots \ldots$ iii

Summary $\ldots \ldots \ldots \ldots \ldots \ldots \ldots \ldots \ldots \ldots \ldots \ldots \ldots$

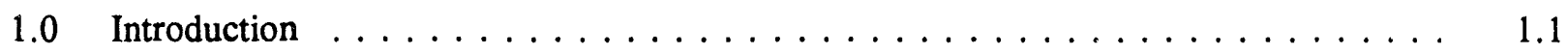

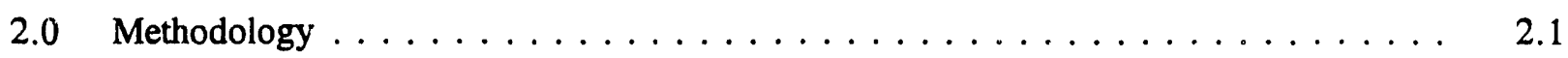

2.1 Foods Important to Dose $\ldots \ldots \ldots \ldots \ldots \ldots \ldots \ldots \ldots \ldots \ldots \ldots$

2.2 Local Consumption Data $\ldots \ldots \ldots \ldots \ldots \ldots \ldots \ldots \ldots \ldots \ldots \ldots$

2.3 National Consumption Data $\ldots \ldots \ldots \ldots \ldots \ldots \ldots \ldots \ldots$

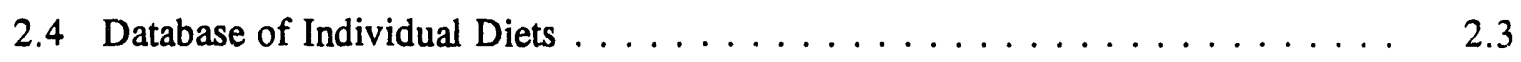

2.5 Aggregating Types of Food $\ldots \ldots \ldots \ldots \ldots \ldots \ldots \ldots$

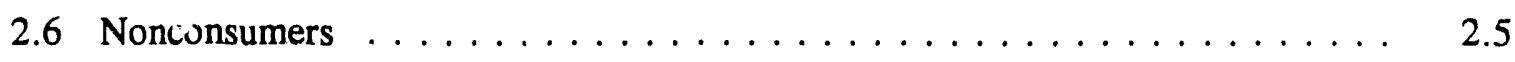

2.7 Backcasting Ratios $\ldots \ldots \ldots \ldots \ldots \ldots \ldots \ldots \ldots \ldots \ldots \ldots$

2.7.1 Disappearance Data . . . . . . . . . . . . . 2.5

2.7.2 Ratio Calculations . . . . . . . . . . . . . . 2.6

2.8 Database Backcasting Ratios $\ldots \ldots \ldots \ldots \ldots \ldots \ldots \ldots \ldots \ldots$

2.9 Reliability of Backcasting $\ldots \ldots \ldots \ldots \ldots \ldots \ldots$

2.10 Classification and Subsetting of the 1977-1978 NFCS Individual Data . . . . . 2.9

$2.10 .1 \mathrm{Sex} /$ Age/Nursing Classification $\ldots \ldots \ldots \ldots \ldots \ldots \ldots$

2.10 .2 Geographic Classification $\ldots \ldots \ldots \ldots \ldots \ldots \ldots \ldots$

2.10 .3 Urbanization Classification . . . . . . . . . . . . . 2.11

2.10 .4 Seasonal Classification $\ldots \ldots \ldots \ldots \ldots \ldots \ldots \ldots \ldots \ldots$

2.11 Statistical Analysis of the Classifications $\ldots \ldots \ldots \ldots \ldots \ldots \ldots \ldots$ 


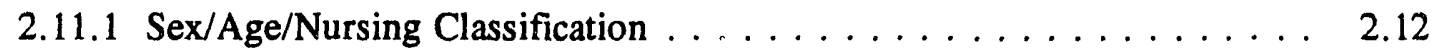

2.11 .2 Geographic Classification $\ldots \ldots \ldots \ldots \ldots \ldots \ldots \ldots$

2.11.3 Urbanization Classification . . . . . . . . . . . . . . 2.13

2.11 .4 Seasonal Classification $\ldots \ldots \ldots \ldots \ldots \ldots \ldots \ldots$

2.12 Average Daily Consumption . . . . . . . . . . . . . . 2.13

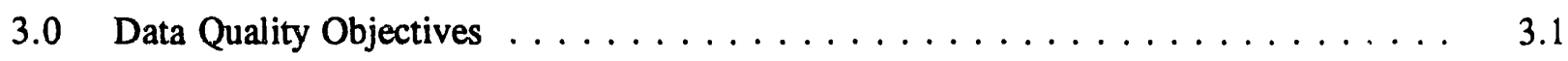

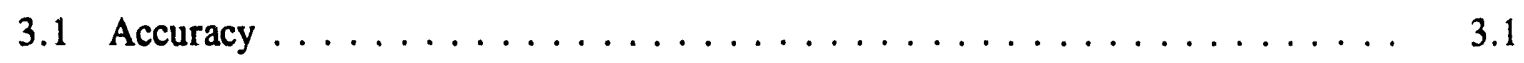

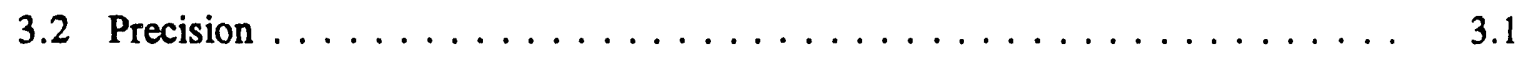

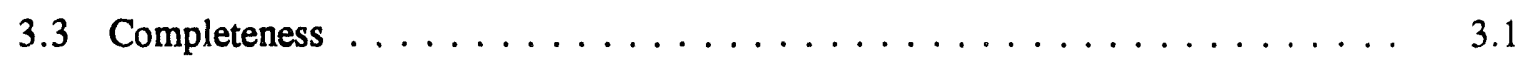

3.4 Representativeness . . . . . . . . . . . . . . . . . 3.1

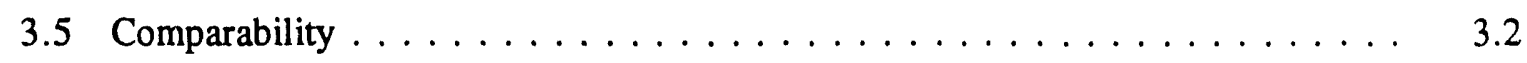

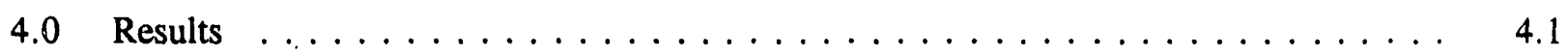

4.1 Human Milk $\ldots \ldots \ldots \ldots \ldots \ldots \ldots \ldots \ldots \ldots \ldots \ldots$

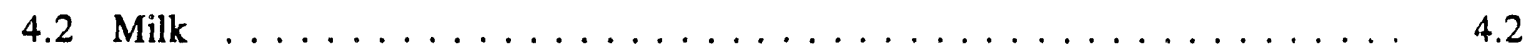

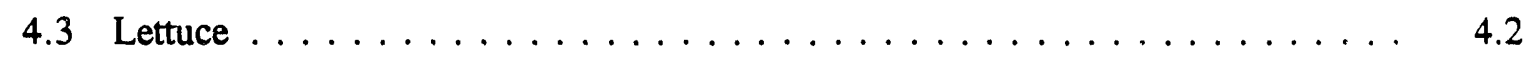

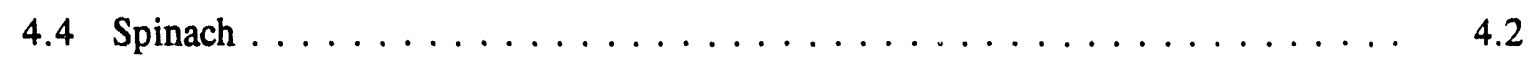

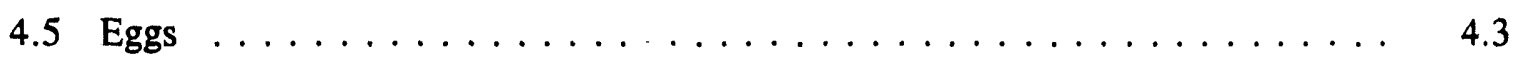

4.6 General Note on Distribution Shapes $\ldots \ldots \ldots \ldots \ldots \ldots \ldots . \ldots \ldots$

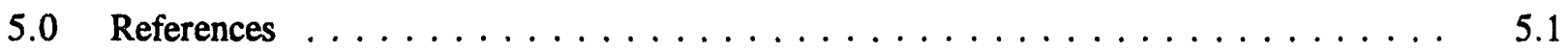

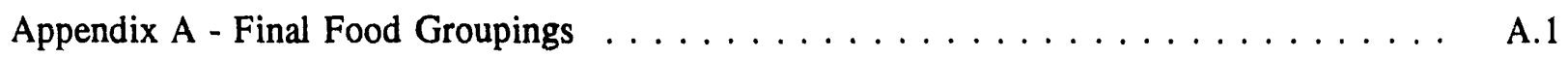

Appendix B - Backcasting 1977-1978 Consumption Estimates to 1945-1957 . . . . . . . B.1

Appendix C - Summary Consumption Statistics $\ldots \ldots \ldots \ldots \ldots \ldots \ldots$ 


\section{Figures}

1.1 The Hanford Environmental Dose Reconstruction Project Study Area . . . . . . . . . 1.2

4.1 Individuals Not Consuming Milk During a 3-Day Period, from 1977-1978

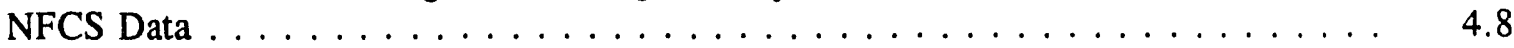

4.2 Distribution of Fresh Milk Consumption for Ages 20-34, from 1977-1978

NFCS Data . . . . . . . . . . . . . . . . . . . . . . . . .

4.3 Distribution of Fresh Milk Consumption for Ages 10-14, from 1977-1978

NFCS Data . . . . . . . . . . . . . . . . . . . . . . . . 4.9

4.4 Seasonal Distribution of Fresh Milk Consumption for Females, Ages 20-34, from 1977-1978 NFCS Data . . . . . . . . . . . . . . . . . . . . . . . . . . . . 4.10

4.5 Seasonal Distribution of Fresh Milk Consumption for Males, Ages 20-34, from

4.6 Seasonal Distribution of Fresh Milk Consumption for Females, Ages 10-14, from $1977-1978$ NFCS Data . . . . . . . . . . . . . . . . . . . . . . . . 4.12

4.7 Seasonal Distribution of Fresh Milk Consumption for Males, Ages 10-14, from

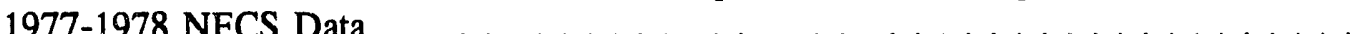

4.8 Distribution of Fresh Milk Consumption by Urbanization for Ages 20-34, from 1977-1978 NFCS Data . . . . . . . . . . . . . . . . . . . . . . . . . .

4.9 Distribution of Fresh Milk Consumption by Urbanization for Ages 10-14, from 1977-1978 NFCS Data.

4.10 Individuals Not Consuming Lettuce During a 3-Day Period, from 1977-1978 NFCS Data

4.11 Distribution of Lettuce Consumption for Ages 20-34, from 1977-1978 NFCS

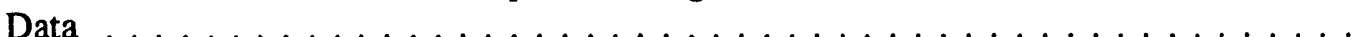

4.12 Distribution of Lettuce Consumption for Ages 10-14, from 1977-1978

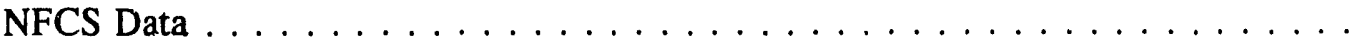

4.13 Seasonal Distribution of Lettuce Consumption for Males, Ages 20-34, from 1977-1978 NFCS Data 
4.14 Seasonal Distribution of Lettuce Consumption for Females, Ages 20-34, from 1977-1978 NFCS Data . . . . . . . . . . . . . . . . . . . . . 4.23

4.15 Seasonal Distribution of Lettuce Consumption for Males, Ages 10-14, from 1977-1978 NFCS Data . . . . . . . . . . . . . . . . . . . . . . . 4.24

4.16 Seasonal Distribution of Lettuce Consumption for Females Ages 10-14, from 1977-1978 NFCS Data . . . . . . . . . . . . . . . . . . . . . . 4.25

4.17 Distribution of Lettuce Consumption by Urbanization for Ages 20-34, from 1977-1978 NFCS Data . . . . . . . . . . . . . . . . . . . . . 4.26

4.18 Distribution of Lettuce Consumption by Urbanization for Ages 10-14, from 1977-1978 NFCS Data . . . . . . . . . . . . . . . . . . . . . 4.27

4.19 Individuals Not Consuming Eggs During a 3-Day Period, from 1977-1978 NFCS Data . . . . . . . . . . . . . . . . . . . . . . 4.36

4.20 Distribution of Egg Consumption for Ages 20-34, from 1977-1978 NFCS Data . . . 4.37

4.21 Distribution of Egg Consumption for Ages 10-14, from 1977-1978 NFCS Data . . . 4.37

4.22 Seasonal Distribution of Egg Consumption for Males, Ages 20-34, from 1977-1978 NFCS Data . . . . . . . . . . . . . . . . . . . . . 4.38

4.23 Seasonal Distribution of Egg Consumption for Females, Ages 20-34, from 1977-1978 NFCS Data . . . . . . . . . . . . . . . . . . . . . . . .

4.24 Seasonal Distribution of Egg Consumption for Males, Ages 10-14, from 1977-1978 NFCS Data

4.25 Seasonal Distribution of Egg Consumption for Females, Ages 10-14, from 1977-1978 NFCS Data

4.26 Distribution of Egg Consumption by Urbanization for Ages 20-34, from 1977-1978 NFCS Data . . . . . . . . . . . . . . . . . . . .

4.27 Distribution of Egg Consumption by Urbanization for Ages 10-14, from 1977-1978 NFCS Data 


\section{Tables}

2.1 Average Daily Milk Consumption Estimates for Children 5-14 Years of Age,

from Local and National Sources for 1965-1969 . . . . . . . . . . . . . . . . . . .

2.2 Theoretical Example - 1945 Database of Individual Diets Providing Information on

Demographics and Food Consumption in the 3-Day NFCS Survey Period . . . . . . .

2.3 1954 Per Capita Food Consumption by Source, Based on Data Presented in USDA

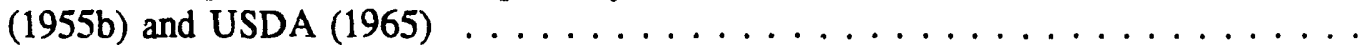

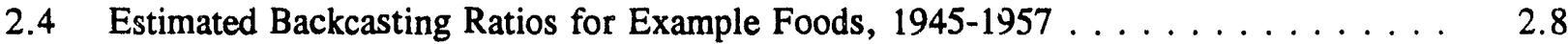

2.5 Backcasting Ratios Used to Convert the Values in the Database of Individual

Diets (DID) to the Year of Interest $(1945-1957) \ldots \ldots \ldots \ldots \ldots . \ldots \ldots$

2.6 Sex, Age, and Nursing Status Classifications $\ldots \ldots \ldots \ldots \ldots \ldots \ldots \ldots$

2.7 Geographic Divisions According to the $1977-1978$ NFCS $\ldots \ldots \ldots \ldots \ldots \ldots \ldots$

4.1 1957-1962 Human Milk Consumption in the First Six Months of Life, from

Data Presented in Durbin et al. (1970) . . . . . . . . . . . . . . . . 4.2

4.2 1977-1978 NFCS Distribution Statistics for Milk Consumption in the Spring $\ldots \ldots .4$

4.3 1977-1978 NFCS Distribution Statistics for Milk Consumption in the Summer . . . 4.5

4.4 1977-1978 NFCS Distribution Statistics for Milk Consumption in the Fall $\ldots \ldots$. . 4.6

4.5 1977-1978 NFCS Distribution Statistics for Milk Consumption in the Winter . . . . 4.7

4.6 1977-1978 NFCS Distribution Statistics for Lettuce Consumption in the Spring . . . . 4.16

4.7 1977-1978 NFCS Distribution Statistics for Lettuce Consumption in the Summer _ . 4.17

4.8 1977-1978 NFCS Distribution Statistics for Lettuce Consumption in the Fall $\ldots \ldots$. . 4.18

4.9 1977-1978 NFCS Distribution Statistics for Lettuce Consumption in the Winter _ . . 4.19

4.10 1977-1978 NFCS Distribution Statistics for Spinach Consumption in the Spring $\ldots \quad \ldots \quad 4.28$

4.11 1977-1978 NFCS Distribution Statistics for Spinach Consumption in the Summer _ . 4.29

$4.121977-1978$ NFCS Distribution Statistics for Spinach Consumption in the Fall $\ldots . .40$

4.13 1977-1978 NFCS Distribution Statistics for Spinach Consumption in the Winter $\ldots . \quad 4.31$ 
4.14 1977-1978 NFCS Distribution Statistics for Egg Consumption in the Spring $\ldots \ldots$. . 4.32

4.15 1977-1978 NFCS Distribution Statistics for Egg Consumption in the Summer $\ldots \ldots .33$

$4.161977-1978$ NFCS Distribution Statistics for Egg Consumption in the Fall $\ldots \ldots$. . . 4.34

4.17 1977-1978 NFCS Distribution Statistics for Egg Consumption in the Winter $\ldots \ldots .35$

4.18 1977-1978 NFCS Annual Daily Average Food Consumption of Foods in the Database of Individual Diets . . . . . . . . . . . . . . . . . 4.36 


\subsection{Introduction}

Part of the dose estimation process in the Hanford Environmental Dose Reconstruction (HEDR) Project involves identifying pathways by which people could have been exposed to radionuclides. One of these exposure pathways is consumption of food that may have contained radionuclides released from Hanford-Site facilities. Thus, estimates are required of the dietary patterns of individuals during the 1940s and 1950s for the area shown in Figure 1.1. These estimates provide the food-consumption information for the HEDR database of individual diets. This database will be an input file in the Hanford Environmental Dose Reconstruction Integrated Code (HEDRIC) computer model that will be used to calculate the radiation dose individuals may have received.

Initial estimates of food consumption for the space and time of interest were provided by Callaway (1992). The purpose of that first study was to demonstrate the feasibility of searching for, evaluating, processing, and/or reconstructing the data needed. The purpose of this final study is to develop the food consumption data necessary to estimate the dose of radiation received from food consumption.

The methodology used in the study was designed to obtain reliable estimates of the diets of individuals living in the HEDR study region with resolution by age, sex, geographic region, and other factors relevant to dose estimation in the computerized model. In general, the methods used build on the work of Callaway and are outlined in Anderson (1992).

Since the initial study, more information has been gathered about which foods are the greatest contributors to dose. The foods of known contribution to dose from iodine-131 are fresh milk and leafy vegetables (PNL 1991). "Leafy" vegetables are defined as any vegetables that have large, exposed, edible surfaces, not just those vegetables with_leaves. Further examination indicated that lettuce and spinach were potentially greater contributors to dose from iodine-131 than all other vegetables and fruits (Marsh et al. 1992). Eggs from free-ranging chickens were also shown to be potentially significant contributors to radiation dose from iodine-131 (Napier 1992b). The level of detail presented by Callaway (1992) was not adequate to reveal consumption estimates for specific foods like spinach or lettuce. Therefore, this study expanded the number of food groups to 65 in order to provide detailed information for the most significant foods.

Although the dose model is not capable of accepting input in detail greater than that of the initial study, this report will show that the reliability of the food aggregation for dose calculation has improved. In the early food consumption analysis described by Callaway (1992), the 1977-1978 Nationwide Food Consumption Survey (NFCS) (USDA 1983) data were aggregaied to 10 food groups, and aggregated trend information was used to convert the consumption values to 1945 . However, in this study, the values to be used as dose model input are derived by first applying specific trend information to 65 individual food groups before performing the aggregation to the level required by the dose model.

The initial study provided consumption estimates for 1945 and 1965 only. This report provides estimates specifically for $1945-1957$. These years were chosen because they were the years of highest 


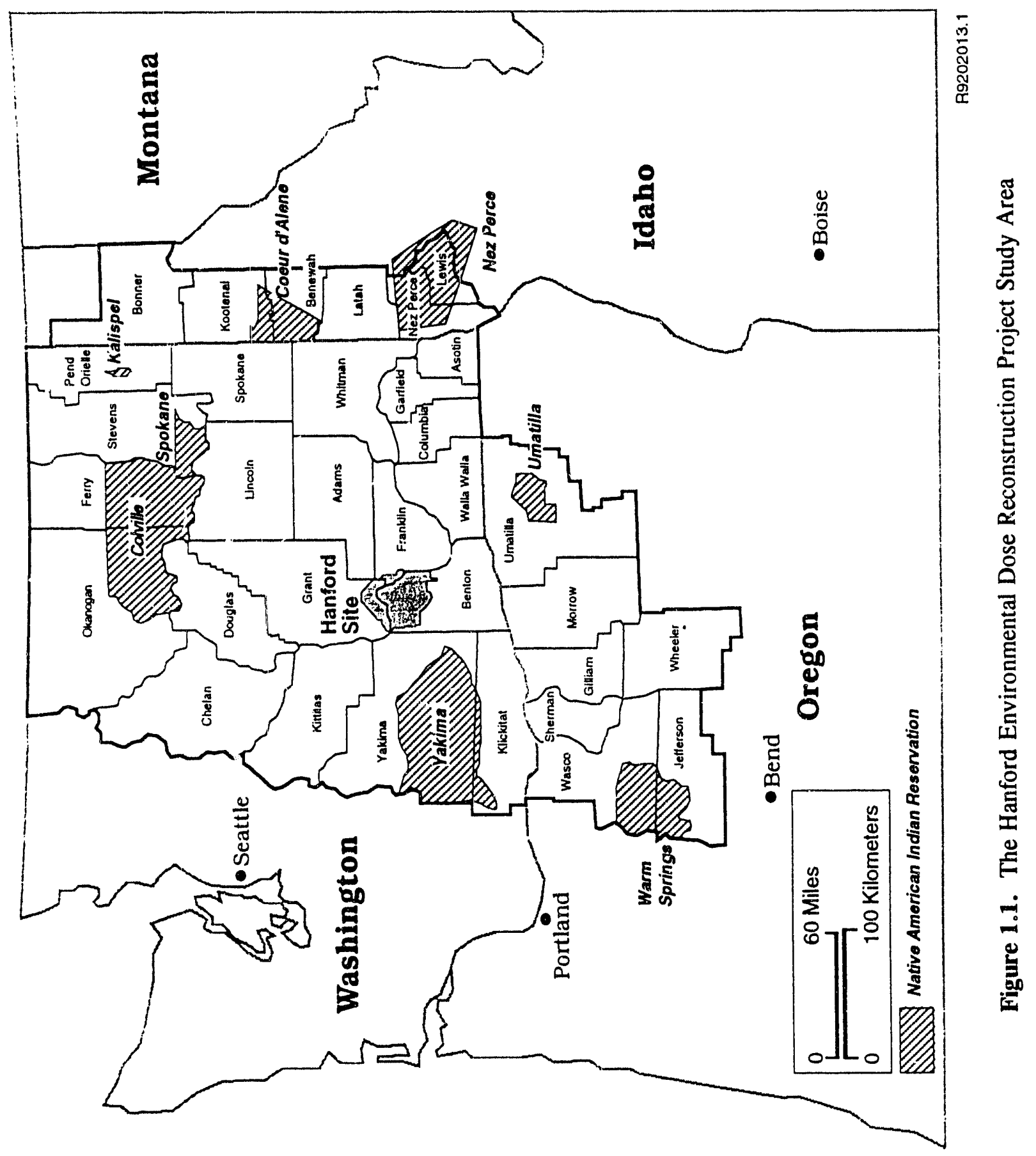


radionuclide releases from Hanford-Site operations and, therefore, the years for which local food production information is being compiled. However, adequately detailed individual raw data on food consumption are not available for the 1945-1957 period. To estimate the food consumption for those years, the 1977-1978 NFCS data were used because they were the most sufficiently detailed individual intake data available. Fictors were developed to backcast the 1977-1978 data based on food-specific consumption trends from the 1940s and 1950s. Information on trends from the 1940s and 1950s was derived from the U. S. Department of Agriculture data (USDA 1965, 1981) on national per capita retail sales quantities. These data series are intact for nearly all of the 65 food groups analyzed. This report also details the backcasting methods used to convert the 1977-1978 values to $1945-1957$ estimates.

The previous study did not include any analysis of inherent uncertainty in the food consumption estimates, nor was any thorough statistical testing performed to improve the subsamples used in the analysis. Such statistical testing and analysis of uncertainty was performed in this study. 


\subsection{Methodology}

The Technical Steering Panel (TSP) methods used in this work generally follow those outlined in Anderson (1992). The analysis consisted of three stages. First, the 3,735 coded food types used in the 1977-1978 NFCS were aggregated to form 75 potential groups for the HEDR analysis. Next, national per capita gross consumption data for the 75 groups were compiled. Finally, 1977-1978 NFCS data were statistically analyzed to produce consumption estimates, which were converted to the years of interest using the estimated conversion factors. Initial analysis of the data revealed there was an insufficient number of observations to merit analyzing 15 of the $\mathbf{7 5}$ food groups. Those 15 groups were apportioned into 5 groups. The result is the 65 food groups as outlined in Appendix A.

\subsection{Foods Important to Dose}

Four specific foods have been identified as potentially significant contributors to radiation dose: milk (PNL 1991), eggs (Napier 1992b), lettuce, and spinach (Marsh et al. 1992). This report provides detailed consumption information for these four foods with summary detail for all other foods.

\subsection{Local Consumption Data}

Available local food-consumption information was reviewed as part of this study but was determined to be not as detailed as national data for the purposes of calculating individual doses. Some of the local consumption data, however, is offered for purposes of comparison with estimates from the analysis.

Bustad and Terry (1956) blended data from several national sources to develop food consumption estimates for 1950 to be used in estimating radiation doses from Hanford releases. That work provided estimates of consumption of milk, flour, fats, eggs, sweets, fresh and processed vegetables, fresh and processed fruits, beef, pork, poultry, and fish. That study adapted farm family survey data on consumption collected by Clark and LeBovit (1955) and can provide a check of the consumption estimates generated in this report. Local (Tri-Cities) fish consumption information (unpublished) collected by Battelle scientists in the late 1960s was used to estimate historic fish consumption in the initial report (Callaway 1992). Battelle collected and published other local (Tri-Cities) food consumption data from that time period. Honstead (1966 and 1967) reported the results of dietary intake data collection that took place during the Hanford-Site whole body radiation counts in 1965 and 1966. Consumption distributions were presented for Tri-Cities residents' consumption of water, milk, coffee, tea, seafood, game iirds, fresh meat, and Columbia River fish. However, these data are of limited precision and accuracy because respondents were asked to provide data in terms of estimated "glasses" of liquid or estimated "meals" of solids. No age or sex distribution of the sample is provided. Soldat and Honstead (1968) studied the diets of elementary school children in the TriCities area of Washington. The information they reported was based on a self-administered 7-day survey taken by 2,973 children, 6-14 years of age. Consumption information was collected on drinking water, milk, other liquids, bread, Columbia River fish, game birds, beef, pork, and seafood. 
The precision and accuracy of these data are suspect because children collected the data and were asked to estimate 8-ounce-equivalent "cups" in the case of liquids and "meals" in the case of solids. Dietary information was gathereat from a total of 5,219 Tri-Cities elementary school children from 1965 through 1968, and the raw data are maintained as an appendix in Endres et al. (1972). Shipler et al. (1972) conducted a study of 341 members of farm families in the Riverview area, west of Pasco, Washington, in 1969. Consumpticin information is provided for milk, fresh vegetables, fresh fruit, game birds, poultry, fish, eggs, leafy vegetables, and water. The data from that report also have been used to check the resulits in this report.

To use the available local consumption information directly would have required additional assumptions about age/sex distributions, glass equivalents, meal equivalents, season of consumption, and representativeness of Tri-Citians compared to the rest of the HEDR region. The raw data on individual consurr.ption from the survey of Tri-Cities household fish consumption, the elementary school children study, and the Riverview study still exist in printed form, but the information is not entirely legible. Although not done for this report, such data could be entered into a computer for analysis with some interpretation of the illegible characters required. Data from these studies were used to check estimates generated using the backcasting approach.

Honstead (1966) pointed out that the local consumption data referred to in his report were collected in conjunction with the earliest efforts to identify potential ingestion pathways and were considered quite preliminary at the time. There are no equivalent local time series data available to identify the changing local trends in consumption. Such information would be required to reliably estimate 1945-1957 consumption from 1965-1969 local data. Lack of reliable and detailed local consumption data resulted in the use of national consumption data.

\subsection{National Consumption Data}

The USDA conducted food consumption surveys pertaining to the period of interest (USDA 1941,1944 , 1955a, 1955b), but these samples were taken during one season of the particular year, were based on household income, not on geographic region, and, therefore, were only comparatively small samples (USDA 1944). In addition, the number and detail of the food types surveyed were not extensive enough to provide the detailed data required to calculate dose.

The USDA conducted the only decennial comprehensive surveys of food consumption on a national level in 1954-1955, 1965-1966, 1977-1978, and 1985-1986. The 1955 NFCS data provide only household-level information. The data are not a useful source for deriving intake estimates of consumption by individuals for the 1945-1955 period because no individual consumption information is provided. However, the 1955 Household Food Consumption Surveys (HFCS) also produced a study on home production of food for home use (USDA 1955b). Although presented at the household level, some of the information has been adapted for application in this study in the cases of milk, leafy vegetables, and eggs, to provide an estimation of "backyard" food consumption of dose-relevant foods. Individual food intake data from one day in spring 1965 form the only data set in existence from the 1965-1966 HFCS (USDA 1972). The 1977-1978 NFCS effort provided an increase in detail and volume of individual intake data collected above that of the 1965-1966 HFCS and was readily available to researchers. Although the 1985-1986 NFCS was also readily available, it was targeted to 
measure food corsumption of low-income households and single mothers in particular and was further removed in time from the period of interest. Therefore, the 1977-1978 NFCS data set was chosen to develop the baseline estimates of consumption. The 1965 HFCS data set was used to perform a sensitivity analysis (see Appendix B).

Table 2.1 provides a comparison of local and national consumption estimates for milk. The 5-14 age group is the only age/sex group common to several studies and has been used for comparison here. This comparison shows that backcasting NFCS 1977-1978 estimates of consumption to the 1965-1969 period for the population groups shown yields a resuit closer to values from local data than does the 1965 HFCS for milk. Because there is no comparable method to convert local consumption estimates to the 1945-1957 period, the comparison to the 1965-1969 period was the only viable check of the backcast estimates against local data.

Table 2.1. Average Daily Milk Consumption Estimates for Children 5-14 Years of Age, from Local and National Sources for 1965-1969

\begin{tabular}{|l|l|c|c|c|c|}
\hline Year & \multicolumn{1}{|c|}{ Data Source } & $\begin{array}{c}\text { Males 5-9 } \\
\text { (grams/day) }\end{array}$ & $\begin{array}{c}\text { Females 5-9 } \\
\text { (grams/day) }\end{array}$ & $\begin{array}{c}\text { Males 10-14 } \\
\text { (grams/day) }\end{array}$ & $\begin{array}{c}\text { Females 10-14 } \\
\text { (grams/day) }\end{array}$ \\
\hline 1967 & Local Data & & & & \\
1969 & Soldat and Honstead (1968) & 700 & 630 & 700 & 640 \\
& Shipler et al. (1972) & 786 & 773 & 858 & 821 \\
& National Data & & & & \\
1965 & HFCS 1965-1966 (USDA 1972) & 620 & 570 & 660 & 560 \\
$1967^{(a)}$ & NFCS 1977-1978 & 770 & 850 & 710 & 700 \\
\hline (a) 1977-1978 NFCS values backcasted to 1967.
\end{tabular}

\subsection{Database of Individual Diets}

The database of individual diets (DID) will be created to provide a source of seasonal dietary information for the HEDR Project food groups from the individual intake data of the 1977-1978 NFCS. This database will be used to reconstruct the diets of reference individuals required for input into the dose calculations for reference individuals. The DID will be comprised of one file of the actual observations from the 1977-I978 NFCS including the 1945-1957 backcasting ratios to convert NFCS values to any year in the 1945-1957 period. The theoretical structure of the DID is shown in Table 2.2. The final format of the DID will be established prior to the completion of the dose code. 
Table 2.2. Theoretical Example--1945 Database of Individual Diets Providing Information on Demographics and Food Consumption in the 3-Day NFCS Survey Period

\begin{tabular}{|c|c|c|c|c|c|c|}
\hline Sex & Age & Season & $\begin{array}{l}\text { Urban/ } \\
\text { Rural }\end{array}$ & $\begin{array}{l}\text { Fresh Milk } \\
\text { (grams) }\end{array}$ & $\begin{array}{c}\cdots \\
\text { (grams) }\end{array}$ & $\begin{array}{l}\text { Poultry } \\
\text { (grams) }\end{array}$ \\
\hline $\bar{M}$ & 21 & $\mathbf{F}$ & $\mathbf{U}$ & 465 & $\ldots$ & 699 \\
\hline $\mathbf{M}$ & 21 & $\mathbf{F}$ & $\mathbf{U}$ & 400 & $\ldots$ & 700 \\
\hline $\mathbf{M}$ & 21 & $\mathbf{F}$ & $\mathbf{U}$ & 444 & $\ldots$ & 688 \\
\hline $\mathbf{M}$ & 55 & W & $\mathbf{R}$ & 401 & $\ldots$ & 688 \\
\hline $\mathbf{M}$ & 55 & W & $\mathbf{R}$ & 333 & $\ldots$ & 707 \\
\hline $\mathbf{M}$ & 55 & W & $\mathbf{R}$ & 350 & $\ldots$ & 655 \\
\hline F & 12 & Sp & $\mathbf{R}$ & 355 & $\ldots$ & 551 \\
\hline F & 12 & Sp & $\mathbf{R}$ & 0 & $\ldots$ & 505 \\
\hline $\mathbf{F}$ & 12 & Sp & $\mathbf{R}$ & 0 & $\ldots$ & 602 \\
\hline F & 34 & Su & $\mathbf{R}$ & 0 & $\ldots$ & 566 \\
\hline$F$ & 34 & Su & $\mathbf{R}$ & 222 & $\ldots$ & 577 \\
\hline$F$ & 34 & $\mathrm{Su}$ & $\mathbf{R}$ & 304 & $\ldots$ & 540 \\
\hline $\mathbf{M}$ & 5 & $\mathrm{Su}$ & $\mathbf{R}$ & 111 & $\ldots$ & 55 \\
\hline $\mathbf{M}$ & 5 & $\mathrm{Su}$ & $\mathbf{R}$ & 233 & $\ldots$ & 110 \\
\hline $\mathbf{M}$ & 5 & $\mathrm{Su}$ & $\mathbf{R}$ & 400 & $\ldots$ & 77 \\
\hline$F$ & 1 & F & $\mathbf{U}$ & 600 & $\ldots$ & 3 \\
\hline F & 1 & F & U & 454 & $\ldots$ & 11 \\
\hline F & 1 & $\mathbf{F}$ & U & 467 & $\ldots$ & 8 \\
\hline$F$ & 46 & W & $\mathrm{U}$ & 225 & $\ldots$ & 680 \\
\hline$F$ & 46 & W & U & 0 & $\ldots$ & 655 \\
\hline F & 46 & W & $\mathrm{U}$ & 225 & $\ldots$ & 634 \\
\hline $\mathbf{F}$ & 27 & W & $\mathrm{U}$ & 231 & $\ldots$ & 577 \\
\hline F & 27 & W & U & 330 & $\ldots$ & 524 \\
\hline F & 27 & W & $\mathbf{U}$ & 304 & $\ldots$ & 666 \\
\hline
\end{tabular}

\subsection{Aggregating Types of Food}

Food consumption data will be provided to the Environmental Pathways and Dose Estimates Task staff for input into the HEDRIC dose model. The 65 food groups will be aggregated in the DID to the 9 food groups used by HEDRIC. The principal food groups which the HEDRIC dose model uses (Eslinger et al. 1992, p. 23) are as follows:

Fresh cow milk

Stored cow milk

Leafy vegetables

Other vegetables 
Fruit

Grain

Eggs

Beef

Poultry

In the case of mixed foods such as cream pies, creamed vegetables, sandwiches with lettuce, etc., the question was to which of the groups should the food be allocated. This problem was handled using an approach similar to that of Nelson and Yang (1984). The mixed foods with dose-important ingredients were allocated to one of the 65 food groups using an approximation of the proportion of the components in the mixed foods. This exercise was carried out for NFCS mixed foods containing (or likely to contain) fresh milk, cream, lettuce, spinach, and eggs. Special attention was given to these foods because they are known to be the most dose relevant from the food pathway.

\subsection{Nonconsumers}

Information in the DID is provided for each 3-day sequence of consumption information from the 1977-1978 NFCS data and converted to 1945 in the theoretical example shown in Table 2.2. Because the individuals represented in the DID provided three days of consumption data, selecting the entire three-day sequence of consumption from any single sample observation controls the betweenday correlation effect. However, because there are only 3 days of data per individual, it cannot be reliably determined. from NFCS data whether specific individuals are nonconsumers of a specific food. For example, an individual, who did not report consuming milk during the 3-day period of the survey, would be given an average consumption of 0 grams per day in the database, but, in fact, may have consumed milk over a longer time frame than the 3 days covered by the survey.

\subsection{Backcasting Ratios}

Because the 1977-1978 NFCS data were used in this analysis, the estimates generated from the 1977-1978 NFCS data had to be converted to 1945-1957 terms. A food-specific conversion factor called a backcasting ratio was used. Use of these ratios is based on the assumption that national average changes in consumption patterns over time would adequately reflect the dietary changes over time for the 1977-1978 subsample that included Oregon and Washington.

\subsubsection{Disappearance Data}

It is important to note that data denoting national per capita consumption are measured in terms of estimated "disappearance" of retail quantities into the marketing and distribution system. By definition, home-produced food is not measured in retail disappearance data. It can be reasonably concluded that in the 1940s and 1950s in the HEDR study region individuals consumed significant quantities of home-produced food. These foods could include fresh milk and cream from the 
backyard cow, fresh produce such as lettuce and spinach from the garden, and eggs from the backyard chicken, among many other foods. Although home-produced food is not measured in disappearance data, it is assumed that trends reflected in retail sales quantities are trends for all food, whether the source is retail or a home product.

However, it was possible, using USDA (1955b), to evaluate whether those eating homeproduced foods were likely to consume more of those foods than individuals who consumed from retail sources only. Table 2.3 compares per capita consumption by source, whether retail or home produced. It appears that individuals consuming home-produced supplies of milk and leafy vegetables consumed nearly 20 percent more of those foods in 1954 than individuals consuming milk and leafy vegetables from retail sources. Retail egg consumption was higher than home-produced egg consumption. This may reflect that households producing eggs at home supplemented their egg consumption from retail sources.

Table 2.3. 1954 Per Capita Food Consumption by Source, Based on Data Presented in USDA (1955b) and USDA (1965)

\begin{tabular}{|l|c|c|c|}
\hline $\begin{array}{c}\text { 1954 Household } \\
\text { Food Source }\end{array}$ & Milk (lbs/yr) & Eggs (lbs/yr) & $\begin{array}{c}\text { Leafy Vegetables } \\
\text { (lbs/yr) }\end{array}$ \\
\hline Home produced & 378.4 & 37.2 & 55.1 \\
Retail & 317.9 & 47.4 & 45.9 \\
Home surplus & 60.5 & -10.2 & 9.2 \\
Home/retail ratio & 1.19 & 0.78 & 1.20 \\
\hline
\end{tabular}

\subsubsection{Ratio Calculations}

Backcasting ratios were derived using the per capita retail sales quantities data series maintained by USDA $(1965,1981)$ to apply changing trends in consumption over time to the 1977-1978 estimates. These data series are consistent and intact for the analysis period being considered.

However, Manchester and Farrell (1981) describe what potential improvements in the series data would enhance their data reliability for use in food consumption analysis. They point out that, while the time series data are plentiful and comprehensive at the farm (producer) level, data on end-use consumption are compiled from sources with purposes other than measuring food consumption. Appendix B provides a characterization of the uncertainty inherent in the per capita consumption data. 
The backcasting ratio for a specific food type is given by the formula:

$$
R_{i_{x}}=\frac{D_{i_{x}}}{D_{i_{77}}}
$$

where

$$
\begin{aligned}
& R_{i} \quad=\text { the backcascing ratio for food } \mathrm{i} \text { for year } \mathrm{x} \\
& D_{i} \quad=\text { per capita consumption (retail disappearance) of food } \mathrm{i} \\
& x \quad=\text { year of interest in the 1945-1957 time period. }
\end{aligned}
$$

The conversion factor was applied using the formula:

$$
\hat{C}_{i_{77}} \cdot R_{i_{x}}=\hat{C}_{i_{x}}
$$

where

$$
\begin{array}{ll}
\hat{C}_{i_{77}} & =\text { estimated consumption of fond } \mathrm{i} \text { from NFCS data } \\
R_{i_{x}} & =\text { backcasting ratio for food } \mathrm{i} \text { for year } \mathrm{x}(1945-1957) \\
\hat{C}_{i_{x}} & =\text { estimated consumption of food } \mathrm{i} \text { in year } \mathrm{x}(1945-1957) .
\end{array}
$$

The backcasting ratios used for fresh milk, eggs, lettuce, and spinach are displayed in Table 2.4. Multiply the values in Table 2.4 by 1977-1978 NFCS consumption estimates to obtain estimated consumption in the reference year. This procedure was used to derive the mean consumption values shown in Tables C.61-C.63 of Appendix C. This procedure can be illustrated using a hypothetical example of 1945 lettuce consumption. If the NFCS data reveal that a specific individual consumed a daily average of $20 \mathrm{~g}$ of lettuce in 1977 , the estimated 1945 daily lettuce consumption for that individual would be calculated:

$$
20 g \cdot 0.6=12 g
$$

\subsection{Database Backcasting Ratios}

In the case of the diet database to be used for dose calculation, backcasting ratios were estimated for the aggregated food groupings required in HEDRIC. This was accomplished using the formula shown below. Table 2.5 presents the backcasting ratios to be used in the HEDRIC dose model. The ratios of Table 2.4 differ slightly from those in Table 2.5 because of the aggregation process. 
Table 2.4. Estimated Backcasting Ratios for Example Foods (1945-1957)

\begin{tabular}{|c|c|c|c|c|}
\hline Year & Milk & Eggs & Lettuce & Spinach \\
\hline 1945 & 1.79 & 1.40 & 0.60 & 1.69 \\
1946 & 1.73 & 1.32 & 0.67 & 1.76 \\
1947 & 1.64 & 1.36 & 0.68 & 1.46 \\
1948 & 1.58 & 1.39 & 0.65 & 1.40 \\
1949 & 1.58 & 1.38 & 0.62 & 1.55 \\
1950 & 1.59 & 1.41 & 0.64 & 1.39 \\
1951 & 1.62 & 1.43 & 0.64 & 1.55 \\
1952 & 1.64 & 1.43 & 0.69 & 1.40 \\
1953 & 1.62 & 1.39 & 0.69 & 1.35 \\
1954 & 1.63 & 1.38 & 0.70 & 1.09 \\
1955 & 1.64 & 1.36 & 0.75 & 1.18 \\
1956 & 1.65 & 1.35 & 0.79 & 1.28 \\
1957 & 1.63 & 1.33 & 0.77 & 1.16 \\
\hline
\end{tabular}

$$
R_{A x}=\frac{\sum_{i=1}^{n} D_{i_{x}}}{\sum_{i=1}^{n} D_{i_{77}}}
$$

where

$$
\begin{aligned}
& \mathrm{R}_{A}=\text { backcasting ratio for any database aggregate } \\
& \mathrm{D}_{i}=\text { national per capita consumption of food } \mathrm{i} \text { in year } \mathrm{x} \\
& \mathrm{n}=\text { number of foods to be aggregated in a single database aggregate food } \\
& \mathrm{x}=\text { the year of interest in the } 1945-1957 \text { period. }
\end{aligned}
$$

\subsection{Reliability of Backcasting}

Appendix B provides a characterization of the inherent uncertainty that the backcasting method introduces into the food consumption estimates. In general, measurement and statistical error generated in the conversion from farm production quantities to retail sales quantities is the source of the most uncertainty passed on to any particular backcasting ratio. The calculation used to determine retail disappearance is described in Appendix B. 
Table 2.5. Backcasting Ratios Used to Convert the Values in the Database of Individual Diets (DID) to the Year of Interest (1945-1957)

\begin{tabular}{|l|c|c|c|c|c|c|c|c|c|c|c|c|c|}
\hline DID Food Type & 1945 & 1946 & 1947 & 1948 & 1949 & 1950 & 1951 & 1952 & 1953 & 1954 & 1955 & 1956 & 1957 \\
\hline Fresh milk & 1.77 & 1.72 & 1.63 & 1.57 & 1.57 & 1.58 & 1.61 & 1.62 & 1.60 & 1.61 & 1.62 & 1.63 & 1.61 \\
Stored milk & 0.92 & 1.12 & 1.05 & 0.98 & 0.98 & 1.00 & 0.96 & 0.98 & 0.97 & 0.97 & 0.99 & 0.98 & 0.97 \\
Leafy vegetables & 1.15 & 1.13 & 1.04 & 1.04 & 0.99 & 1.00 & 0.98 & 0.97 & 0.97 & 0.95 & 0.95 & 0.99 & 0.97 \\
Other vegetables & 1.13 & 1.13 & 1.06 & 0.99 & 0.98 & 1.00 & 0.99 & 0.96 & 0.98 & 0.98 & 0.98 & 0.98 & 1.01 \\
Fruit & 1.39 & 1.49 & 1.50 & 1.40 & 1.41 & 1.24 & 1.33 & 1.26 & 1.22 & 1.19 & 1.10 & 1.14 & 1.11 \\
Grain & 1.35 & 1.29 & 1.16 & 1.14 & 1.13 & 1.12 & 1.11 & 1.09 & 1.06 & 1.04 & 1.02 & 1.01 & 0.99 \\
Eggs & 1.40 & 1.32 & 1.36 & 1.39 & 1.37 & 1.41 & 1.43 & 1.43 & 1.39 & 1.37 & 1.36 & 1.35 & 1.33 \\
Beef and pork & 0.71 & 0.74 & 0.75 & 0.70 & 0.70 & 0.70 & 0.66 & 0.70 & 0.76 & 0.76 & 0.79 & 0.81 & 0.77 \\
Poultry & 0.49 & 0.45 & 0.42 & 0.42 & 0.45 & 0.48 & 0.51 & 0.52 & 0.52 & 0.54 & 0.51 & 0.57 & 0.60 \\
\hline
\end{tabular}

\subsection{Classification and Subsetting of the 1977-1978 NFCS Individual Data}

The 1977-1978 NFCS individual data were analyzed to determine if all of the data or only a selected subset should be kept and whether or not certain subpopulations should be defined. This analysis assured that only the most pertinent data to the HEDR Project were used for a particular individual in the reconstruction of food consumption for dose estimation purposes.

The 1977-1978 NFCS data contain records for each food item consumed over a 3-day period by approximately 30,000 individuals as well as relevant information that can be used to provide different groupings or subpopulations of these individuals. The 1977-1978 NFCS did not collect consumption data for different ethnic groups. Information that was expected to have the greatest potential effect on the usability of the data for reconstructing consumption is listed below:

- Sex of the individual

- Age of the individual

- Nursing status of children and mothers

- Region of the country

- Time of the year

- Degree of urbanization.

\subsubsection{Sex/Age/Nursing Classification}

The first three items listed above were used to create one classification scheme for the individuals in the survey. It was generally expected that these three items would not only lead to very different consumption patterns but also contribute to an individual's physiological response once food was ingested. Accordingly, Table 2.6 depicts how the individuals were grouped into the sub populations which were selected to agree with the groupings defined by Napier et al. (1992, Table 2.1). 


\subsubsection{Geographic Classification}

The most detailed geographic information available for individuals in the 1977-1978 NFCS data were the geographic divisions shown in Table 2.7.

The Pacific division, even though it includes California, would be expected to be the most representative for the HEDR study region. The data were analyzed to see if consumption in other regions of the country was sufficiently similar to consumption in the Pacific region to be included in the HEDR subset as either representative or sufficiently different so that it should be excluded.

Table 2.6. Sex, Age, and Nursing Status Classifications

\begin{tabular}{|c|c|c|}
\hline Sex & Age & Nursing Status \\
\hline Male \& Female & NA ${ }^{\mathrm{a}}$ & Suckling children \\
$"$ & 0 to 6 months & NA \\
$"$ & 7 to 11 months & NA \\
Male & 1 to 4 years & NA \\
$"$ & 5 to 9 years & NA \\
$"$ & 10 to 14 years & NA \\
$"$ & 15 to 19 years & NA \\
$"$ & 20 to 34 years & NA \\
Female & $>34$ years & NA \\
$"$ & 5 to 9 years & NA \\
$"$ & 10 to 14 years & NA \\
$"$ & 15 to 19 years & NA \\
$"$ & 20 to 34 years & NA \\
$"$ & $>34$ years & NA \\
& NA & Pregnant/Nursing \\
\hline (a) NA = Not Applicable. & \\
\hline
\end{tabular}


Table 2.7. Geographic Divisions According to the 1977-1978 NFCS

\begin{tabular}{|l|l|}
\hline \multicolumn{1}{|c|}{$\begin{array}{c}\text { Geographic } \\
\text { Division }\end{array}$} & \multicolumn{1}{c|}{ States Included } \\
\hline New England & Maine, New Hampshire, Vermont, Connecticut, Rhode Island \\
Middle Atlantic & New York, New Jersey, Pennsylvania \\
East North Central & Ohio, Illinois, Indiana, Wisconsin, Michigan \\
West North Central & Minnesota, Iowa, Missouri, North Dakota, South Dakota, Nebraska, Kansas \\
South Atlantic & $\begin{array}{l}\text { Maryland, Delaware, District of Columbia, Virginia, West Virginia, North Carolina, } \\
\text { South Carolina, Georgia, Florida }\end{array}$ \\
East South Central & Kentucky, Tennessee, Alabama, Mississippi \\
West South Central & Arkansas, Louisiana, Texas, Oklahoma \\
Mountain & Montana, Idaho, Wyoming, Utah, Colorado, New Mexico, Arizona, Nevada \\
Pacific & Washington, Oregon, California \\
\hline
\end{tabular}

\subsubsection{Urbanization Classification}

The individuals in the 1977-1978 NFCS data were identified as to the urbanization of the area in which they lived urbanization was defined using a Standard Metropolitan Statistical Area (SMSA) as the point of reference:

Urbanization Level
Central City
Suburban
Nonmetropolitan

\section{SMSA Relationship}

Central city or cities of an SMSA

Within an SMSA but not the central city Not within an SMSA

There was some concern that one or more of these urbanization levels, especially the central city, might not be representative of the populations in the HEDR study area and that urbanization level would have an influence on consumption patterns.

\subsubsection{Seasonal Classification}

Among other areas of investigation, the 1977-1978 NFCS was specifically designed to identify seasonal patterns in food consumption. This classification was analyzed to verify that it was important to consider potential differences in consumption patterns as a result of the time of the year. Although the identical houses were not revisited every quarter (season) during the survey, small neighborhoods, called area segments, were resampled each quarter. The following seasons are defined in the 1977-1978 NFCS: 


\begin{tabular}{ll} 
Season & \multicolumn{1}{c}{ Nominal Period } \\
Spring & Apr, May, Jun 1977 \\
Summer & Jul, Aug, Sep 1977 \\
Fall & Oct, Nov, Dec 1977 \\
Winter & Jan, Feb, Mar 1978
\end{tabular}

\subsection{Statistical Analysis of the Classifications}

A statistical analysis of the classifications described above for the 1977-1978 NFCS individual data was conducted in a manner somewhat similar to the statistical analysis presented in Nelson and Yang (1984). Each of the 65 HEDR food types was analyzed individually. The basic data used as input to the statistical model were average daily total intake for each individual; i.e., the total amount in grams of each HEDR food type an individual consumed divided by the number of days the individual had participated in the survey.

The statistical analysis used was a generalized linear model procedure (SAS 1989). Each of the four classifications listed above was included as a factor in the statistical model as well as each twoand three-factor interaction among the classifications. The full four-factor interaction was not included due to the inability of the analytical software to handle such a large model. The same sampling error term used in Nelson and Yang (1984) was used in testing the hypotheses of this statistical model.

Although each HEDR food type was analyzed separately, it was necessary to reach conclusions about the classifications that would apply in general to all the HEDR food types. In the review of the output from the statistical analysis, most of the conclusions were based on the results for the HEDR fresh milk food type, although the results for all food types were reviewed to arrive at a 'general sense of consistency.

The first conclusion reached from the statistical analysis was that a main-effects analysis-ofvariance model was appropriate; that is, interactions among the main classifications were usually nonsignificant. Accordingly, the 1977-1978 NFCS data were re-analyzed as a main-effects model. Means separation tests (SAS 1989) were used to identify which levels of a classification led to statistically significant differences. The general conclusions reached from these analyses for each of the classifications are summarized below.

\subsubsection{Sex/Age/Nursing Classification}

In general, this classification was by far the most statistically significant. This means that the consumption patterns of individuals are strongly influenced by their age and sex. Therefore, it is important to maintain this classification to form subpopulations in the HEDR food database that will be used to reconstruct consumption. There was no consistent pattern between HEDR food groups as to which of the defined subpopulations of this classification were different from each other so, that there was no obvious utility in collapsing the classification to fewer subpopulations. 


\subsubsection{Geographic Classification}

This classification aıd not appear to be as important as the sex/age/nursing classification but was still statistically significant for many of the HEDR food types. A test was used to compare each of the other geographic divisions to the Pacific division. All divisions were different from the Pacific division for at least one HEDR food type. However, the geographic divisions clearly fell into two groups, those that were diffe ent from the Pacific division on a regular basis and those that were different from the Pacific division only for a small number of the HEDR food types. Those divisions that were distinguishable from the Pacific division were the Scuth Atlantic, East South Central, and West South Cintral divisions. The southeastern part of the country showed consumption patterns that were decidedly cifferent from the Pacific division. Therefore, the southeastern data were not considered representative of the HEDR study region, and they were not included in the HEDR subset of the 1977-1978 NFCS data. All other geographic divisions were included.

\section{2.:1.3 Trbanization Classification}

This classification led to the least definite conclusions of the four classifications. In general, it was the classification that least frequently showed statistically significant differences, alth ugh there viere some. In addition, there was not a consisteni pattern about which of the three levels of urbarization differed from each other. Based on the analyses, the Central City data were removed from the HEDR subset of the 1977-1978 NFCS data. The subpopulation classification of suburban and nonmetropolitan were maintained for the remaining data.

\subsubsection{Seasonal Classification}

The conclusions regarding this classification generally fell into two groups: those where there appeared to be little or no seasonal effect and those where the seasonal effect was quite signi:icant. As expected, the majority of the food types where the seasonal effect was quite significant fell into the general category of fresh produce. Because fresh produce as a group is second only to fresh milk in its iniportance to dose, it is necessary to leave the seasonal classification scheme in the HEDR subset of the 1977-1978 NFCS siata.

\subsection{Averags Daily Consumption}

The concept of average daily consumption for a particular food typically takes two forms:

1. Average daily consumption for all days is calculated by taking an average of all days recorded, including those days (denoted as zero) when that food type was not consumed. This represents the amount consumed on the average.

2. Average daily consumption for those days in which consumption occurred is calculated by taking an average of only those days when consumption was recorded. 
It is relatively simple to convert from one concept to the other if the proportion of days the food was consumed is known.

Let

$$
\begin{aligned}
& \mathrm{ADC}=\text { Average daily consumption over all days } \\
& \mathrm{ADCC}=\text { Average daily consumption on days consumed } \\
& \mathrm{PC}=\text { Proportion of days the food was consumed. }
\end{aligned}
$$

Then $\mathrm{ADC}=\mathrm{PC} * \mathrm{ADCC}$ and $\mathrm{ADCC}=\mathrm{ADC} / \mathrm{PC}$

Example:

$$
\begin{aligned}
& \mathrm{ADC}=4 \\
& \mathrm{ADCC}=10 \\
& \mathrm{PC}=.4
\end{aligned}
$$

$$
\begin{aligned}
& \mathrm{ADC}=\mathrm{PC} * \mathrm{ADCC}=.4 * 10=4 \\
& \mathrm{ADCC}=\mathrm{ADC} / \mathrm{PC}=4 / .4=10
\end{aligned}
$$




\subsection{Data Quality Objectives}

The analysis performed conforms to the data quality objectives outlined in Shipler (1992) and Anderson (1992).

\subsection{Accuracy}

The objective was to develop food consumption estimates that can be used by the HEDRIC dose model to estimate doses for reference individuals in the HEDR study region. The construction of the food group aggregations was overseen by staff of the Environmental Pathways and Dose Estimates and Statistics Tasks to ensure that the foods with the most significant contribution to dose were accurately represented and measured. The Statistics Task staff were involved at various key stages of the analysis to ensure that the statistical procedures used were being employed correctly. Foods included in the database of individual diets were aggregated to the most effective level that the dose model will currently accept.

\subsection{Precision}

The objective was to provide food consumption estimates and a diet database that were estimated and developed using accepted statistical techniques, and that have a quantified level of uncertainty associated. Statistics on the distributions of consumption data were calculated by food group and population group. Associated means, medians, and percentiles were reported for each food group. The conversion factors used to convert 1977-1978 data to the years of interest were calibrated using the most reliable consumption estimates available from raw data. The uncertainty introduced by using backcasting ratios is characterized in Appendix B.

\subsection{Completeness}

The objective was to provide food consumntion estimates and daily diets that apply to representative individuals in the population of the 1945-1957 HEDR study region. Such estimates were developed for all dose-relevant food and population groups. Detailed results are presented for fresh milk, lettuce, spinach, and eggs. Analytical results for other food types, not found to be relevant to dose, are not reported in detail in this report but will be turned over in the DID to the staff of the Environmental Pathways and Dose Estimates Task.

\subsection{Representativeness}

The objective was to develop food consumption estimates for foods likely to be produced in the HEDR study region and most relevant to dose calculation. Based on research to date for the HEDR 
Project, the food groups selected for analysis represent the likely types of food produced and consumed in the HEDR Project region during the study period and are believed to be the most relevant to dose. The foods included in the DID fit this description.

\subsection{Comparability}

The objective was to provide more detailed food consumption information than was presented in the HEDR Phase I effort. Results are presented in greater detail than those reported earlier (Callaway 1992). Resolution has been enhanced by expanding the population groups and food categories to better distinguish types of food and types of people. More descriptive statistics are included. 


\subsection{Results}

The distribution characteristics of dose relevant foods are provided in Tables 4.1-4.17 and Figures 4.1-4.27. Specific information, including plots of the distribution, is presented for milk, lettuce, spinach, and eggs. The number of people surveyed ("No. of Persons") are given in relation to those of that group ("Consumers") who actually consumed the particular food. All consumption numbers reported are in grams per day when food was consumed. Graphic information depicts the characteristics of the consumption distribution for children ages 10-14 and adults ages 20-34. These two population groups were chosen for graphical display because they represent different stages of physiological development. Sample sizes attributable to the information displayed graphically are found by referring to the associated table for the food type being considered. Appendix $C$ provides summary statistics of the other food types analyzed.

The data used in this analysis will be compiled into a database and turned over to the Environmental Pathways and Dose Estimates Task staff to be used as an input file to the dose model. This input file will provide the dose model with the actual observations from the 1977-1978 NFCS and the conversion factors needed to estimate 1945-1957 values. These observations will be used within the dose model to reconstruct a potential sequence of daily diets over time to be used in reconstructing a person's potential ingestion pathways.

The number of true nonconsumers (those who never consume a given food) of any of the foods in the NFCS data cannot be determined from a 3-day record as was collected for the NFCS. The number of nonconsumers presented throughout the results refers only to those individuals who did not consume the given food during the 3-day period of the survey. Medians and averages were calculated based on the days when food was consumed.

The annual daily average consumption of foods as they are listed in the DID appears in Table 4.18. Because the averages are annual, they include all seasons of the year. Days with no consumption are included.

\subsection{Human Milk}

None of the data published by USDA provided human milk intake. The NFCS data provided a classification indicating whether a child was a "suckling child," but no quantities of human milk consumption were given. To provide an estimate of human milk consumption, published estimates for the 1957-1962 period by Durbin et al. (1970) were used. Table 4.1 provides gram/day consumption for each of the first 6 months of life and a weighted average for the 0 to 6 -month period provided in the database of individual diets. 
Table 4.1. 1957-1962 Human Milk Consumption in the First Six Months of Life, from Data Presented in Durbin et al. (1970)

\begin{tabular}{|r|c|c|c|}
\hline $\begin{array}{c}\text { Age } \\
\text { (days) }\end{array}$ & $\begin{array}{c}\text { Number of } \\
\text { Observations }\end{array}$ & $\begin{array}{c}\text { Mean Human Milk } \\
\text { Intake (g/day) }\end{array}$ & $\begin{array}{c}\text { Standard Deviation } \\
\text { (g/day) }\end{array}$ \\
\hline $0-3$ & 30 & 396 & 73 \\
$31-60$ & 42 & 469 & 42 \\
$61-90$ & 33 & 464 & 52 \\
$91-120$ & 42 & 497 & 51 \\
$121-150$ & 39 & 505 & 41 \\
$151-180$ & 36 & 529 & 31 \\
$0-180$ & 222 & 480 & 47 \\
\hline
\end{tabular}

\subsection{Milk}

Analytical results for the consumption of fresh milk are provided in Tables 4.2-4.5 and Figures 4.2-4.5. Nonconsumption of milk is shown in Figure 4.1. Although sample sizes for children in the first year of life are low, the expected shift from breast milk or formula to cow's milk after the first 6 months of life can be inferred. The diminished frequency of milk consumption with increasing age also seems apparent. The differences in milk consumption distributions by sex are demonstrated in Figures 4.2 and 4.3. Differences in milk consumption distributions by season are displayed in Figures 4.4-4.7 for the four age/sex groups being considered. Figures 4.8 and 4.9 denote the differences in milk consumption by urbanization.

\subsection{Lettuce}

Analytical results for the lettuce consumption distribution are provided in Tables 4.6-4.9 and Figures 4.10-4.18. Note that just one individual under the age of 1 year reported consuming lettuce, and approximately 75 percent of those from 1 through 4 years of age did not report any lettuce consumption for the 3-day survey period.

\subsection{Spinach}

Analytical results for the distribution of fresh spinach consumption are provided in Tables 4.10-4.13. In general, 95 percent of the respondents in all population groups did not report consuming any fresh spinach during the 3-day survey period. Because there were so few responses for the population groups being compared graphically, no such graphical display has been presented for spinach consumption. 


\subsection{Eggs}

Information about the consumption distribution for eggs is displayed in Tables 4.14-4.17 and Figures 4.19-4.27. Of the dose-relevant foods considered in this report, eggs are the second most frequently consumed food after milk. However, those reporting no consumption of eggs during the 3-day period generally number in excess of 50 percent.

\subsection{General Note on Distribution Shapes}

In referring to the distribution plots for the foods presented, the reader may notice the somewhat "sawtoothed" shapes of the curves. This shape results from the effect of portion size on the distribution.

For example, consider the distribution of egg consumption. In Figures 4.19-4.27, spikes occur in the distribution plots generally around each 25 - or 50-gram increment along the X-axis. One egg is given a consumed weight of 50 grams (USDA 1979). The distribution plot in this case is picking up the distribution of portions or weight-units within any day in addition to the simple gramconsumption pattern. 


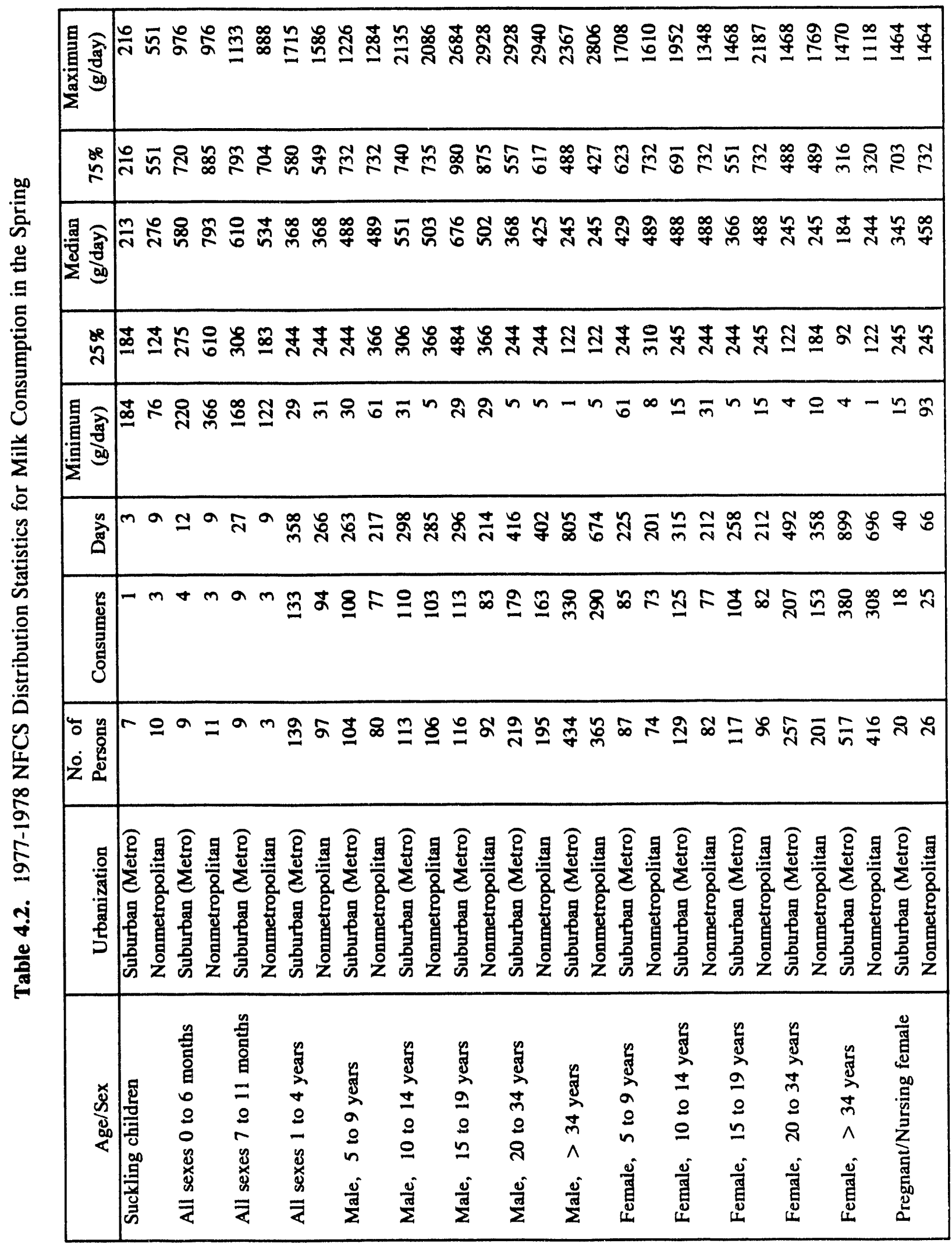

$$
4.4
$$




\begin{tabular}{|c|c|}
\hline 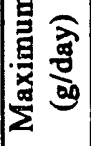 & 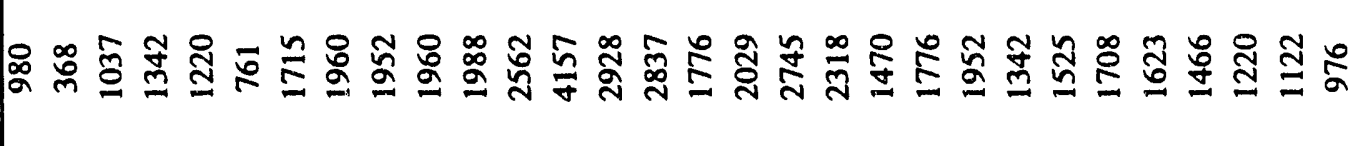 \\
\hline$\stackrel{8}{i}$ & 约 ఓ \\
\hline 迩 & ঢ \\
\hline 果 & 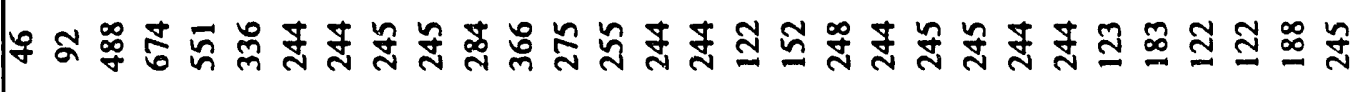 \\
\hline 要 & 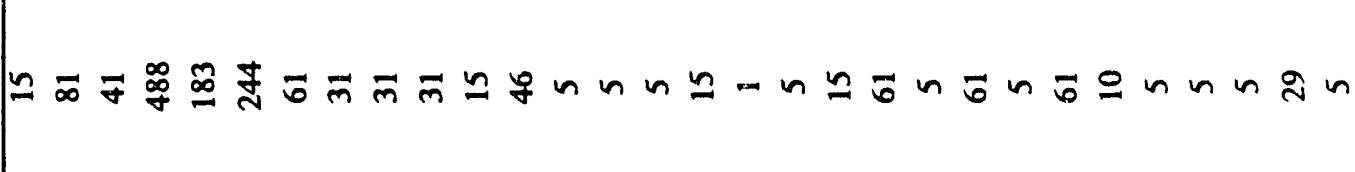 \\
\hline$\stackrel{\text { 离 }}{a}$ & 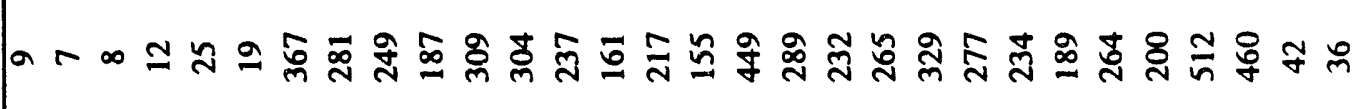 \\
\hline 恶 & 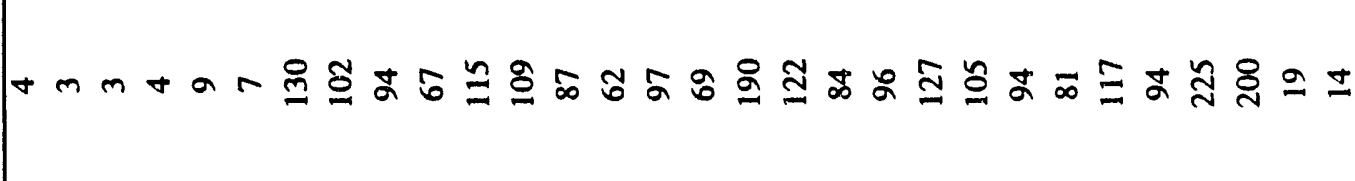 \\
\hline 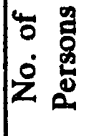 & 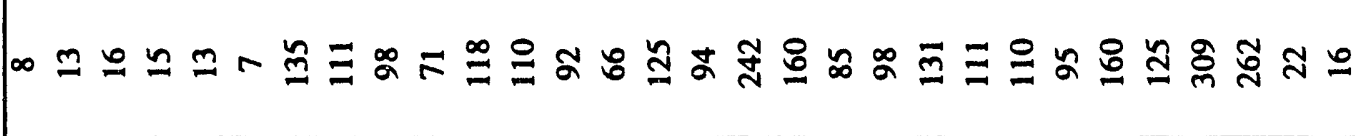 \\
\hline $\begin{array}{l}\text { 売 } \\
\text { 兑 } \\
\end{array}$ & 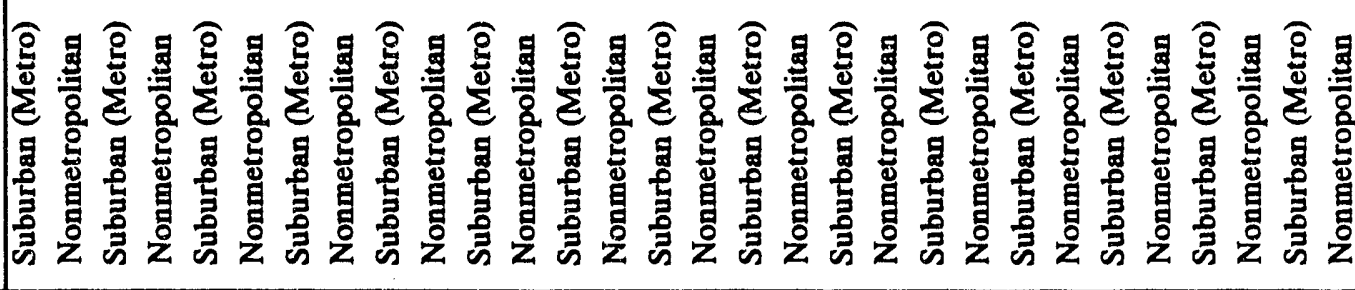 \\
\hline & 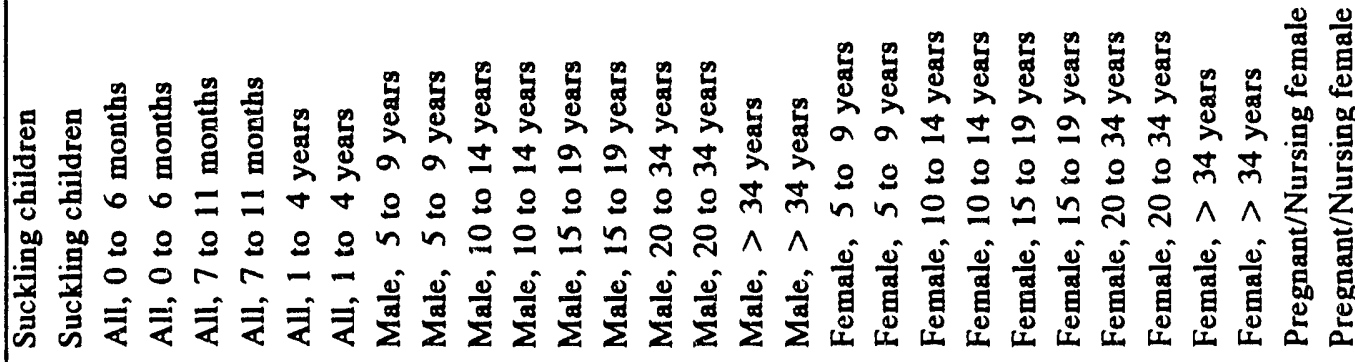 \\
\hline
\end{tabular}




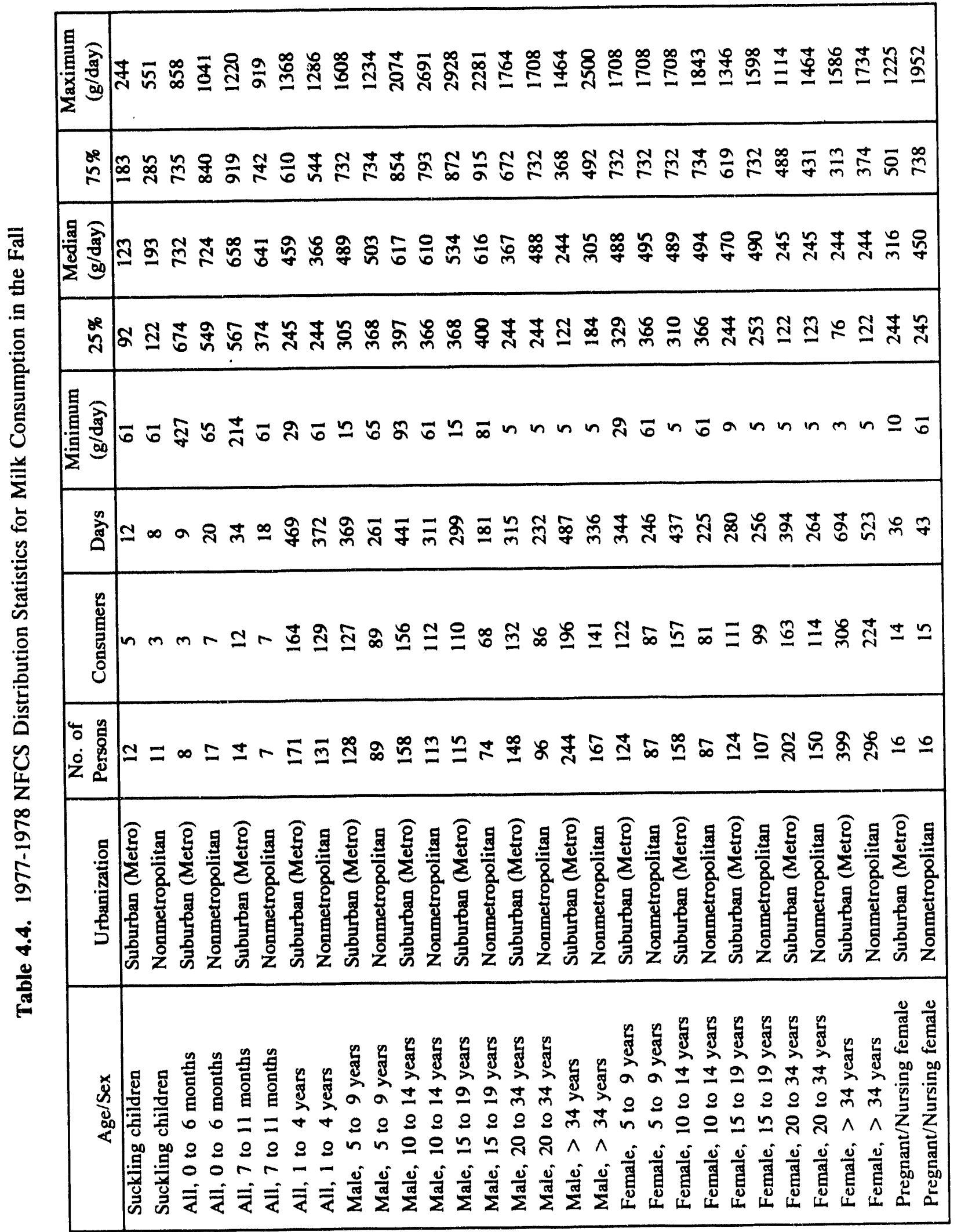




\begin{tabular}{|c|c|}
\hline 要要 & 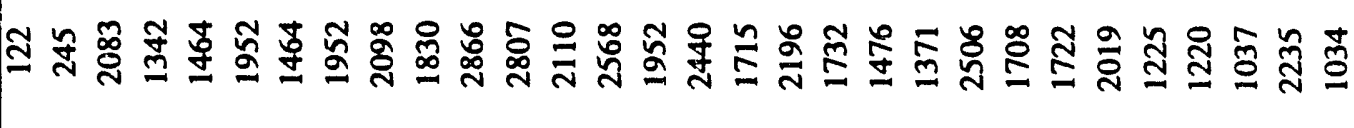 \\
\hline 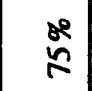 & ఏి \& \\
\hline 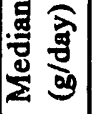 & 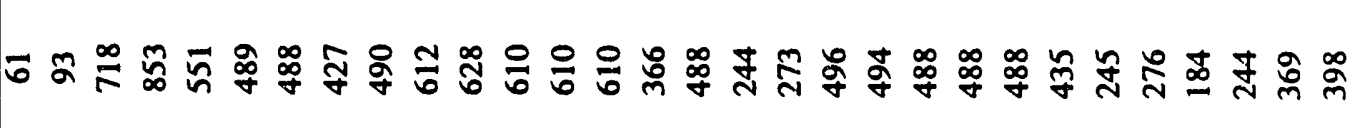 \\
\hline 象 & 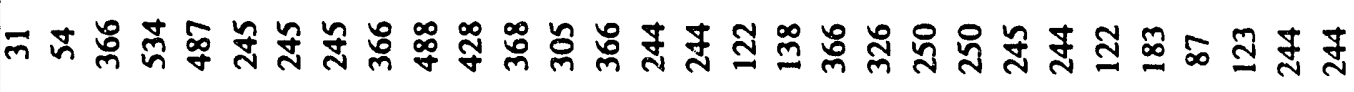 \\
\hline 量 & 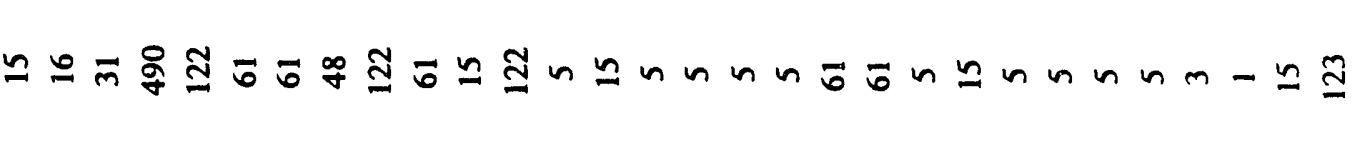 \\
\hline 吾 & 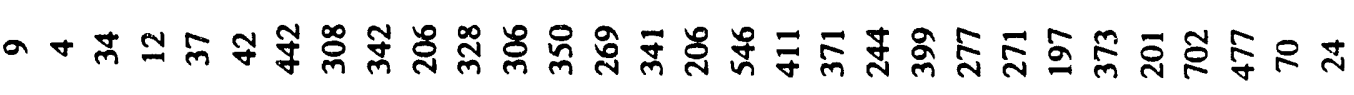 \\
\hline 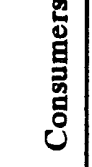 & 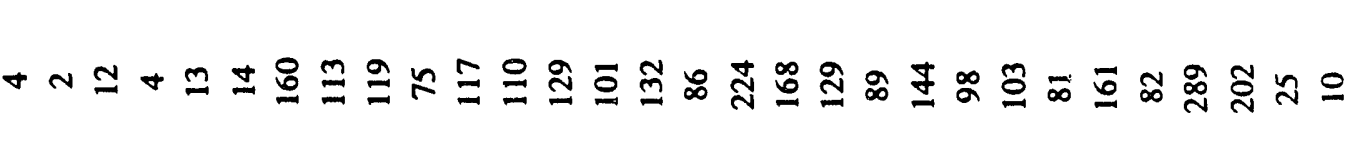 \\
\hline 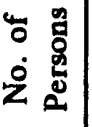 & 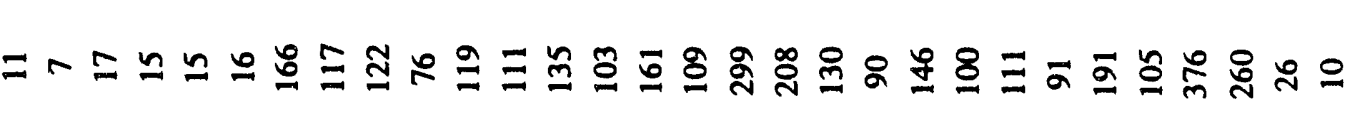 \\
\hline | & 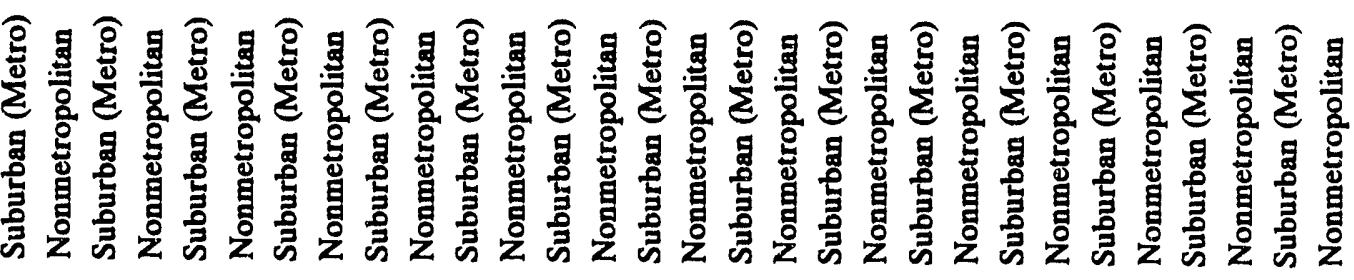 \\
\hline | & 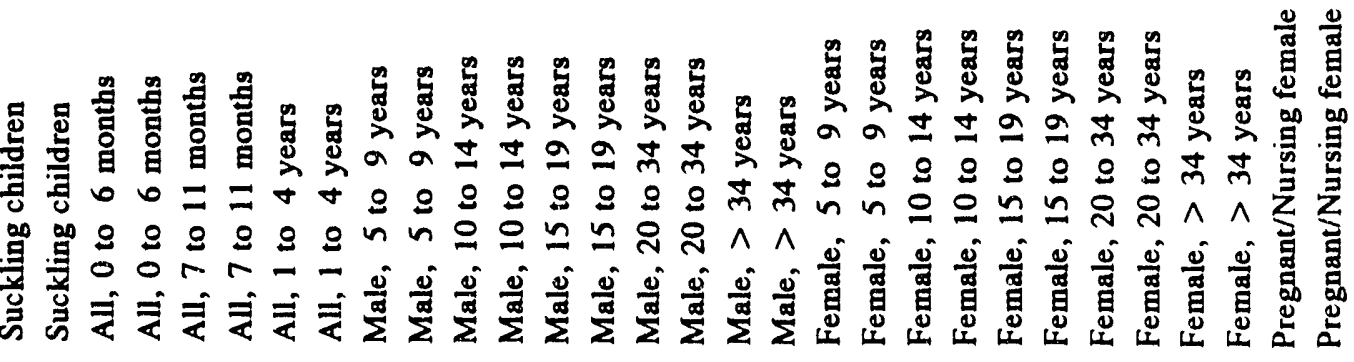 \\
\hline
\end{tabular}




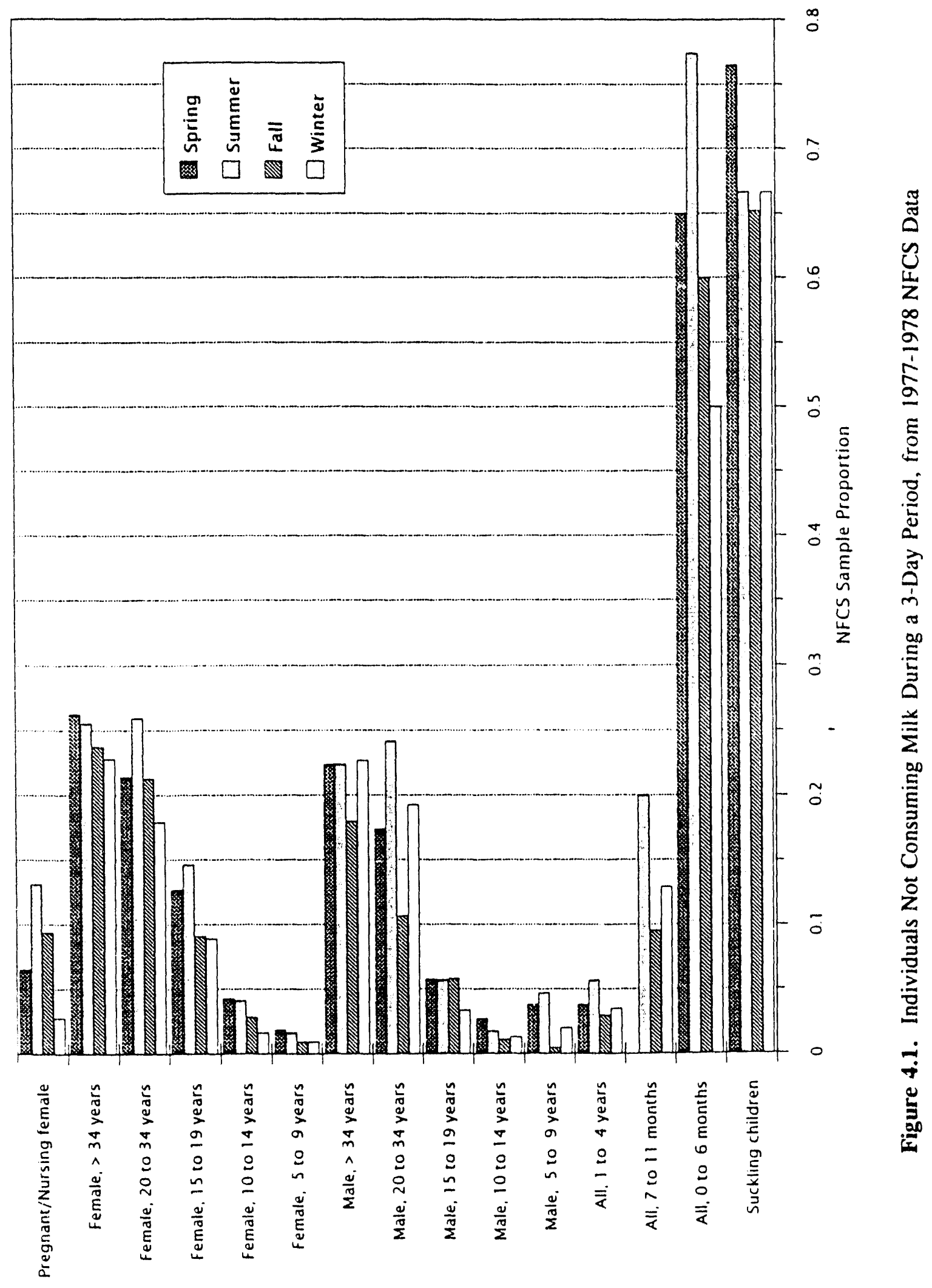




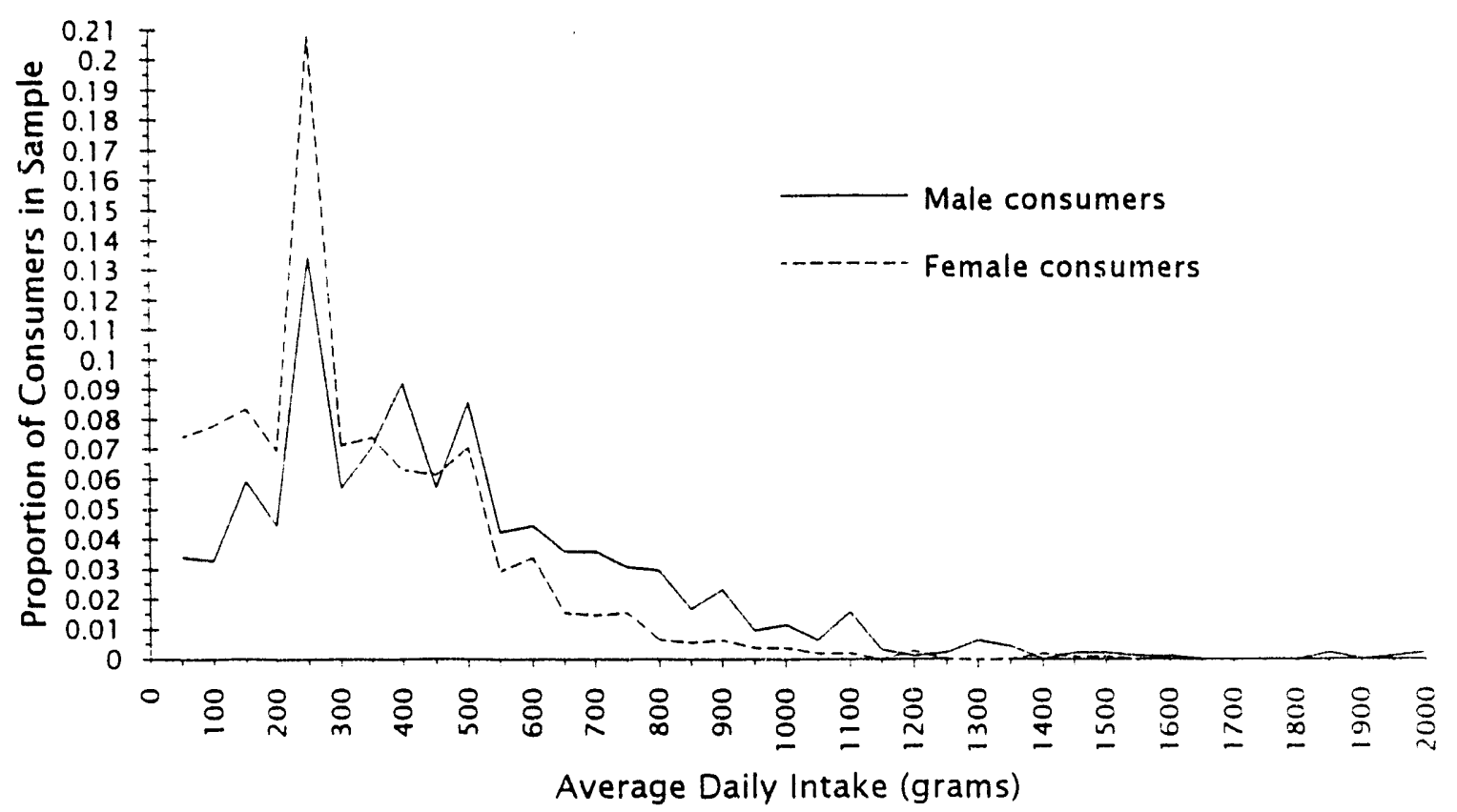

Figure 4.2. Distribution of Fresh Milk Consumption for Ages 20-34, from 1977-1978 NFCS Data

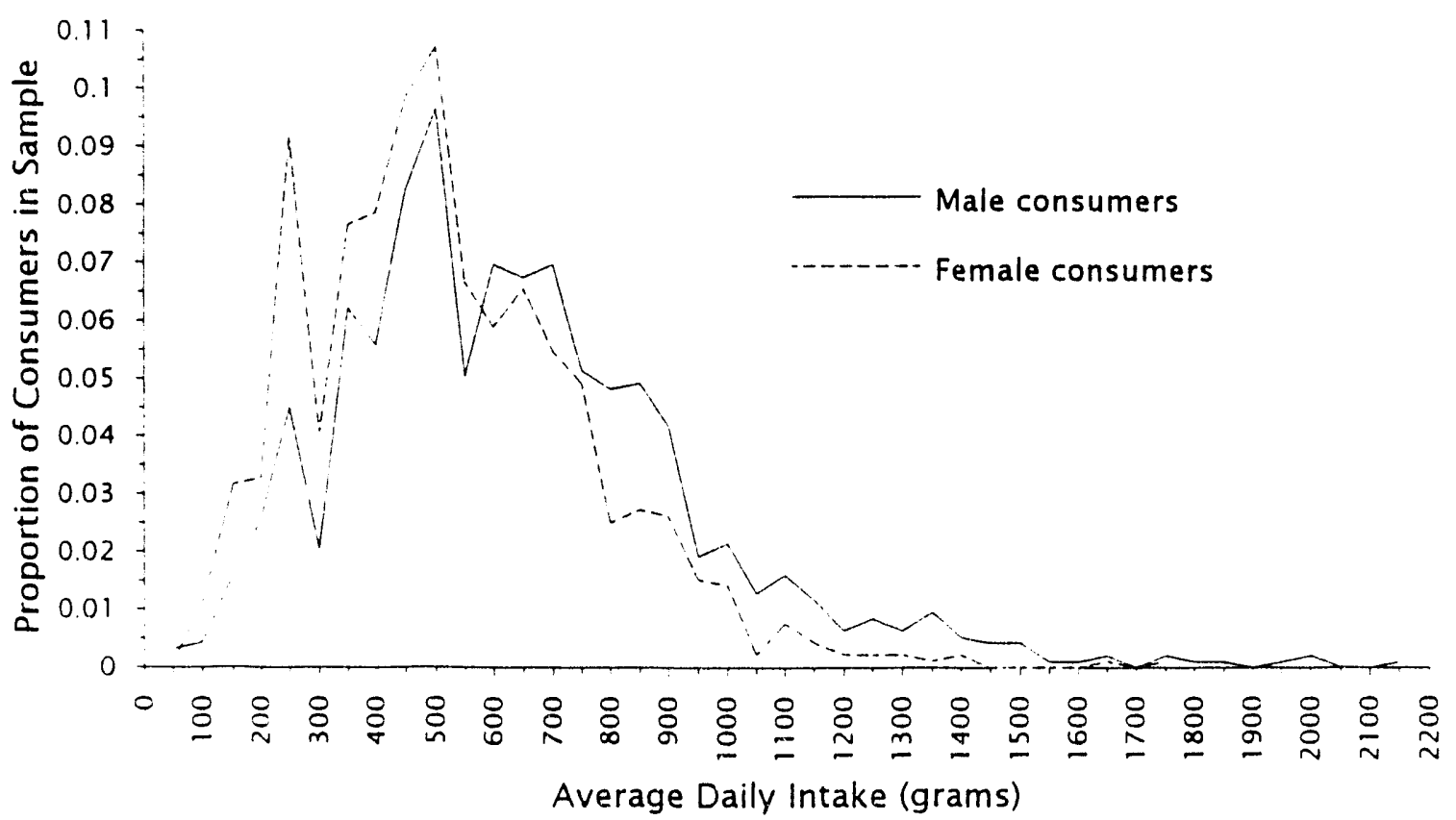

Figure 4.3. Distribution of Fresh Milk Consumption for Ages 10-14, from 1977-1978 NFCS Data 


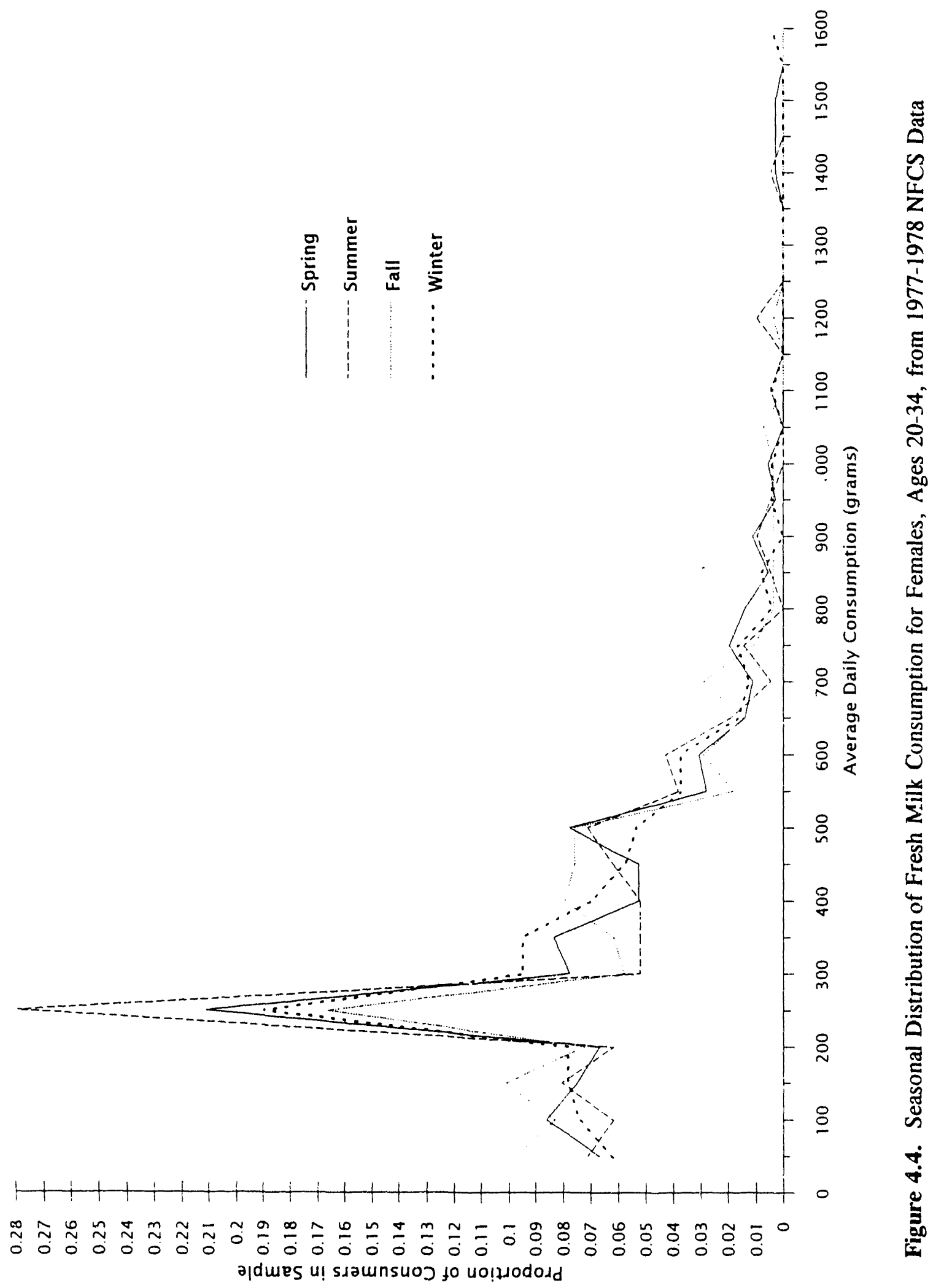




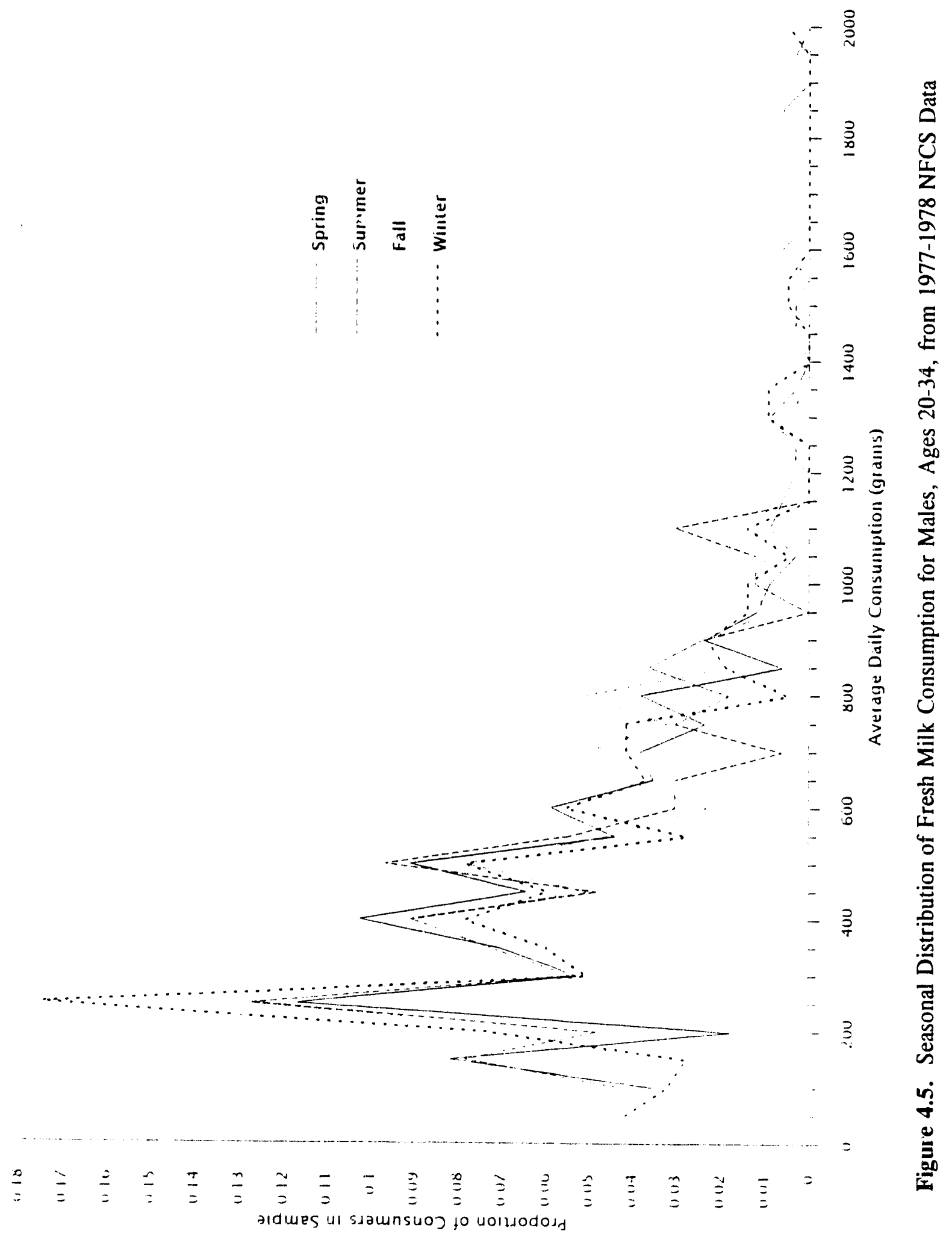

4.11 


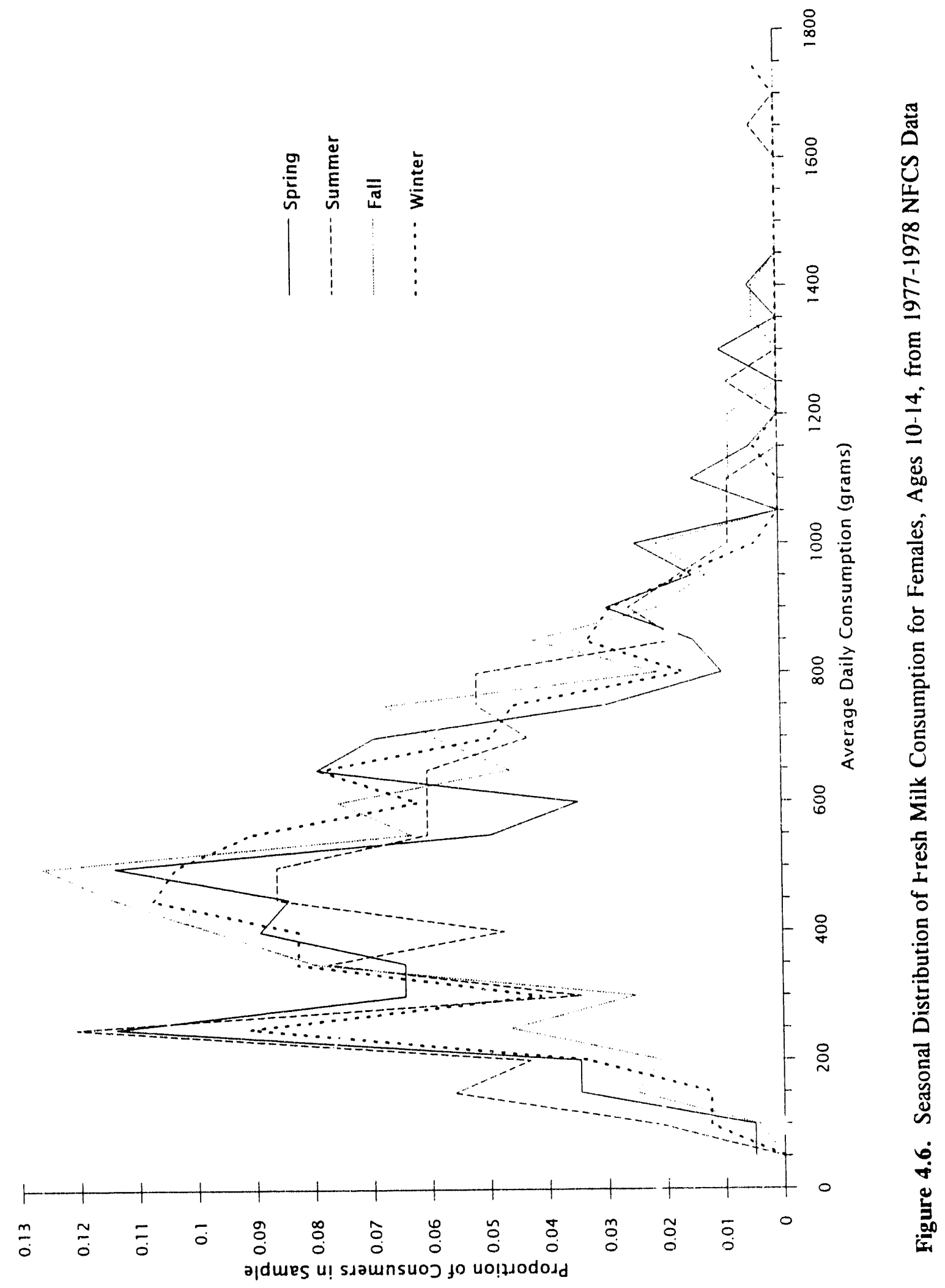




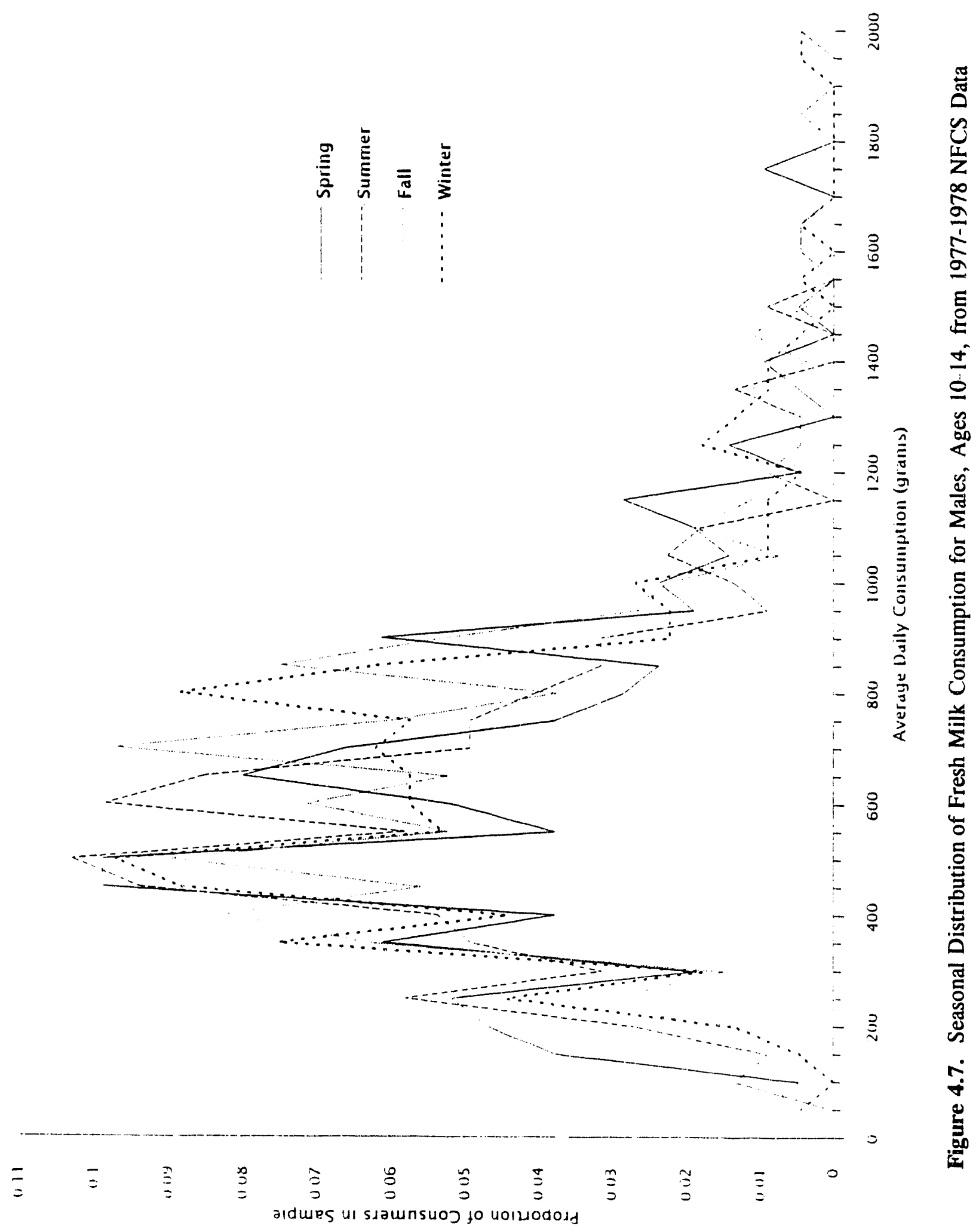

4.13 


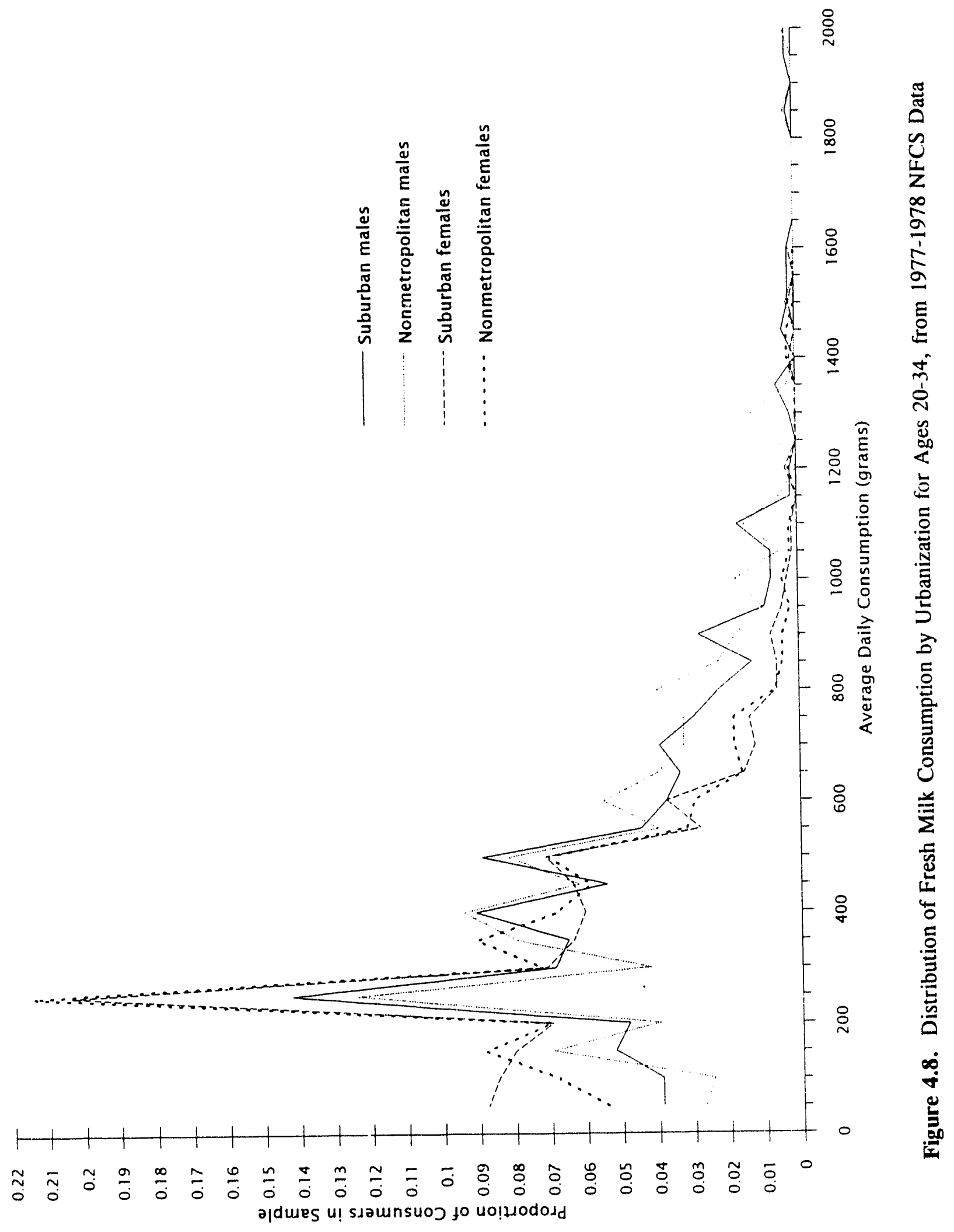

4.14 


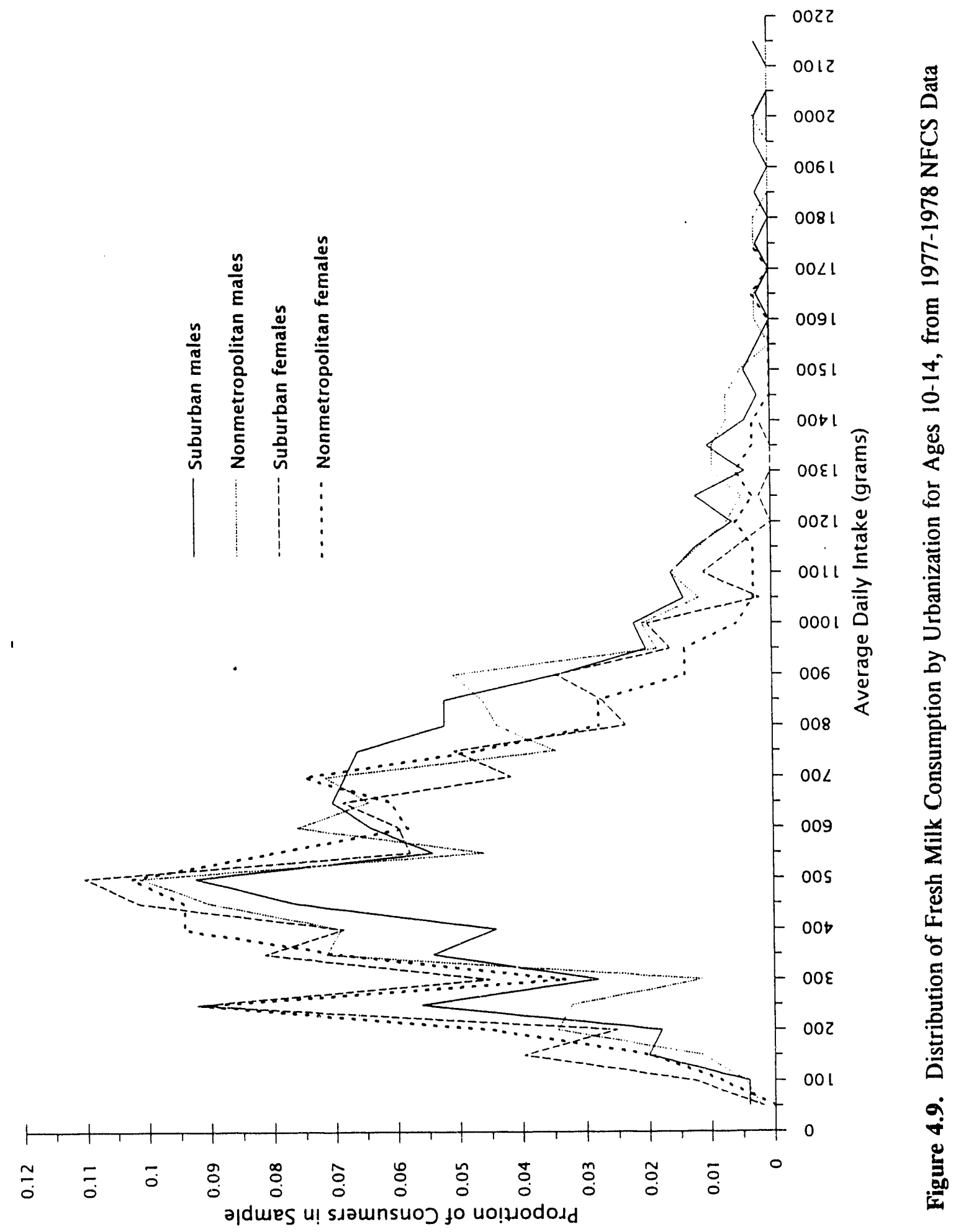




\begin{tabular}{|c|c|}
\hline 悬 & 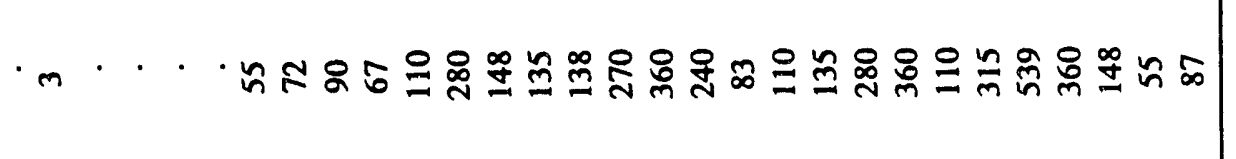 \\
\hline$\stackrel{80}{\approx}$ & 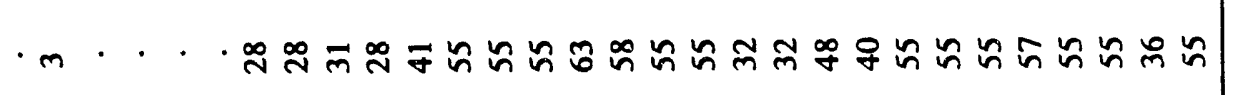 \\
\hline 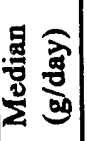 & · ·. \\
\hline$y^{8}$ & 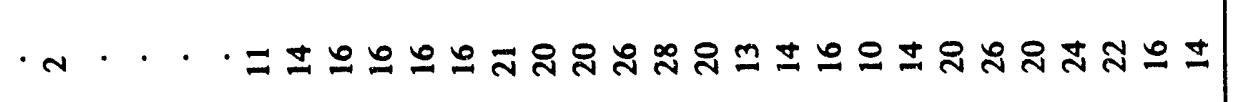 \\
\hline 悬 & 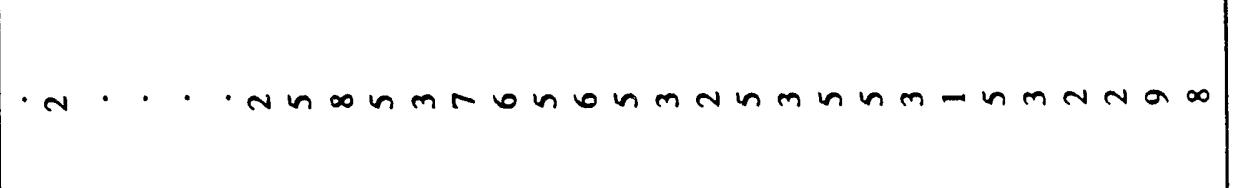 \\
\hline$\stackrel{\infty}{\stackrel{\infty}{\Delta}}$ & 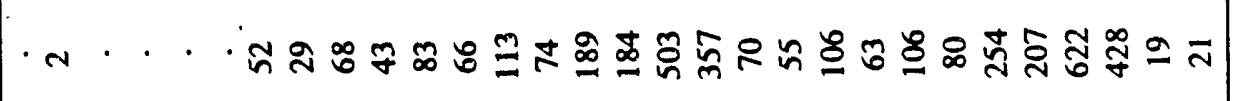 \\
\hline 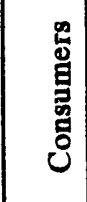 & 0 - \\
\hline 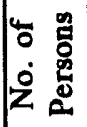 & 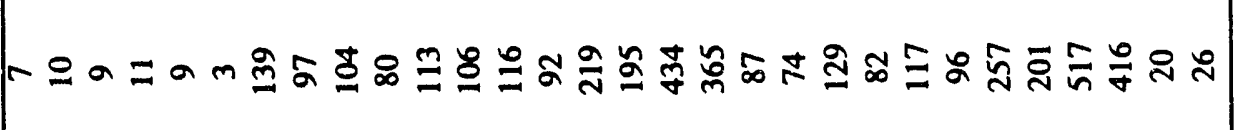 \\
\hline 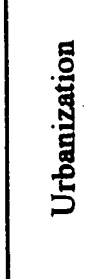 & 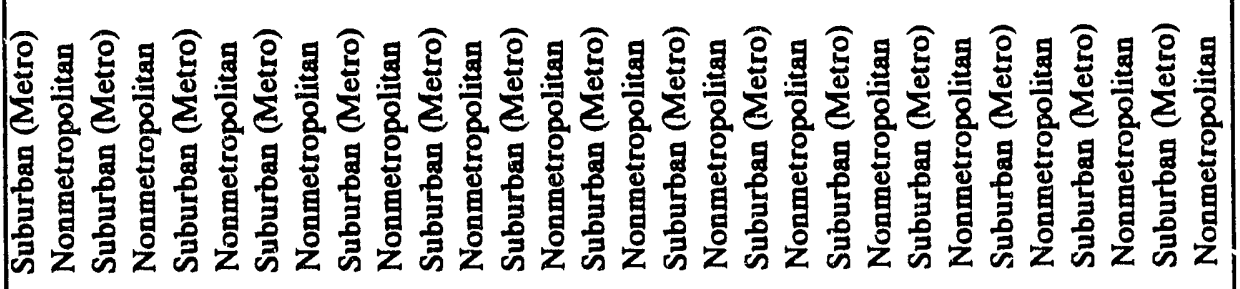 \\
\hline 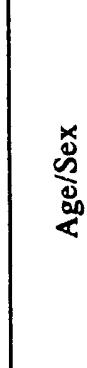 & 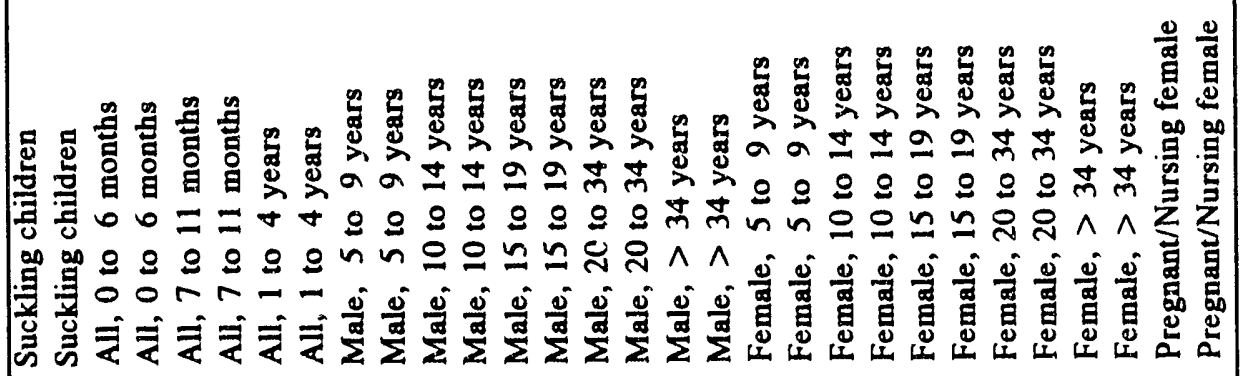 \\
\hline
\end{tabular}




\begin{tabular}{|c|c|}
\hline 道 & 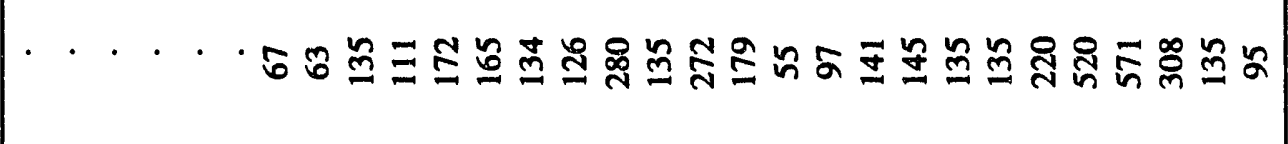 \\
\hline 电 & 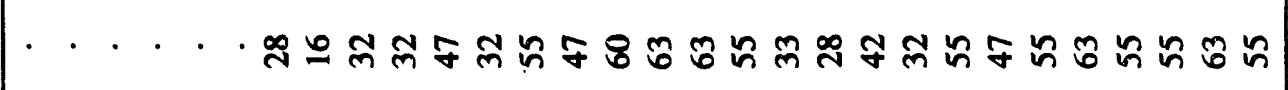 \\
\hline$\frac{1}{2}$ & 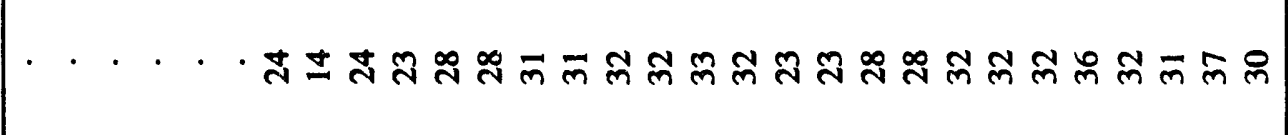 \\
\hline 常 & ·. . . · \\
\hline 离 & 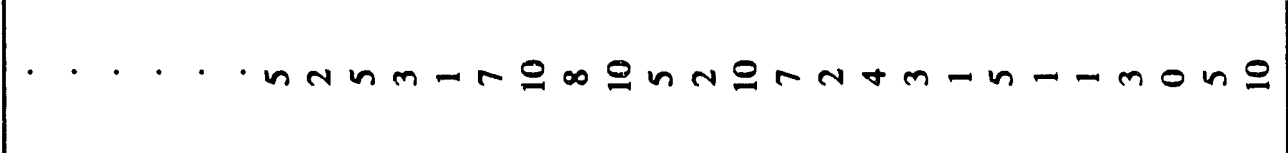 \\
\hline 窅 & 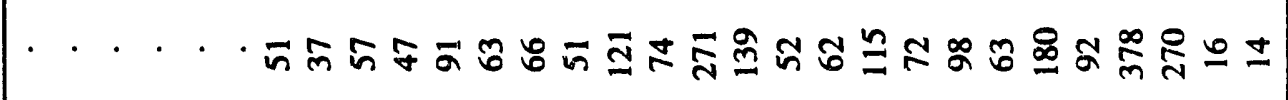 \\
\hline 兽 & 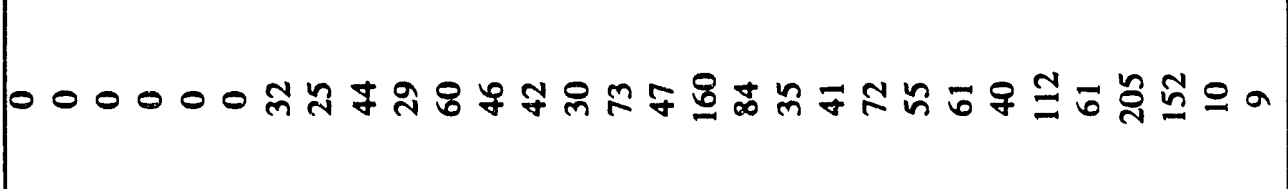 \\
\hline $\begin{array}{l}0 \\
0 \\
0 \\
\dot{0} \\
2 \\
2\end{array}$ & 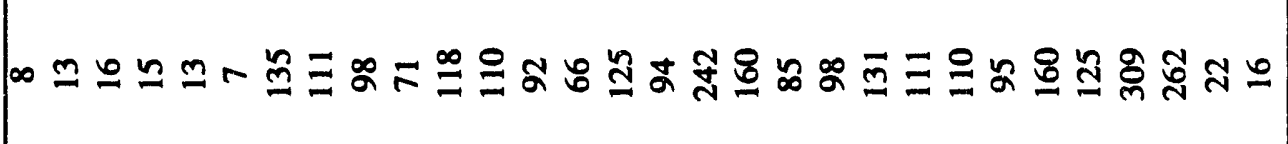 \\
\hline 宽 & 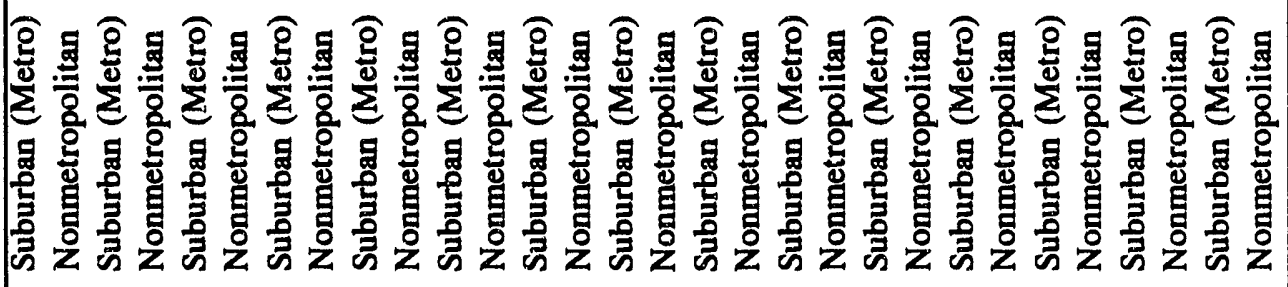 \\
\hline 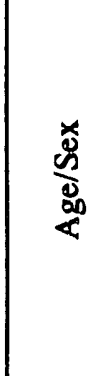 & 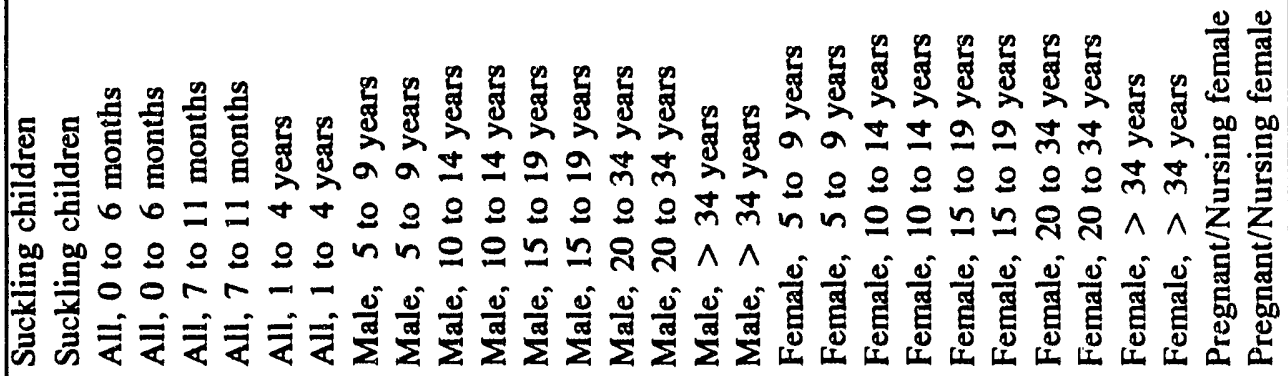 \\
\hline
\end{tabular}




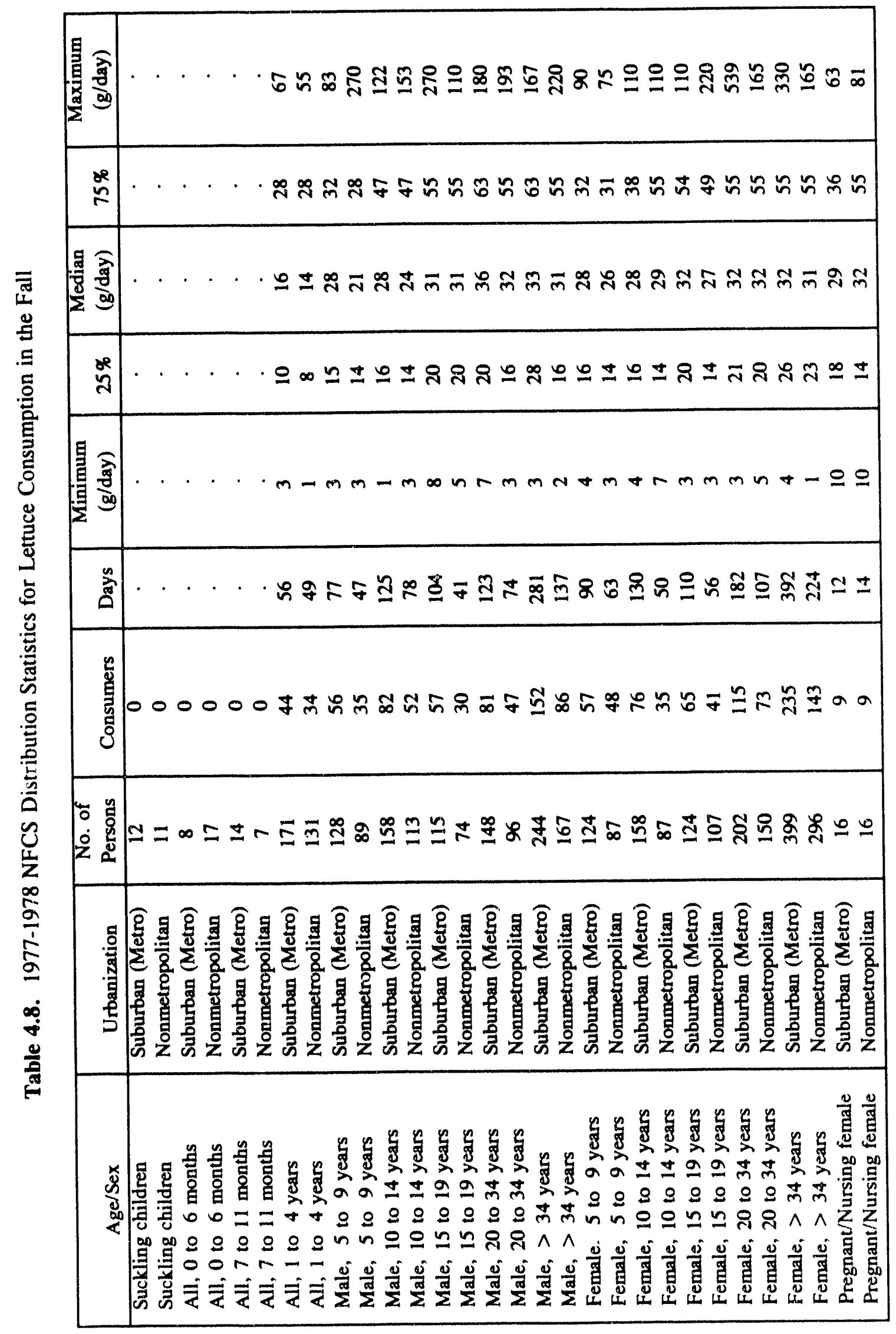




\begin{tabular}{|c|c|}
\hline 焉 & 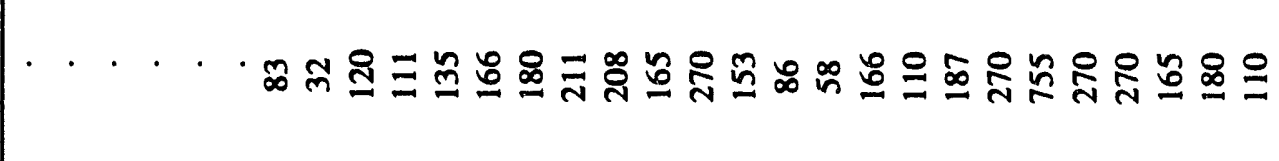 \\
\hline 赵 & 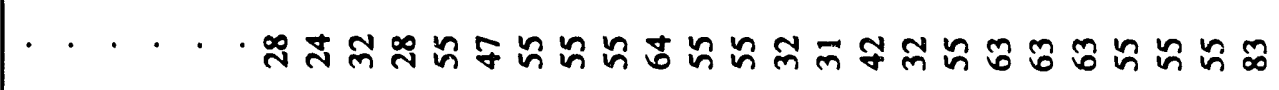 \\
\hline 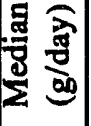 & 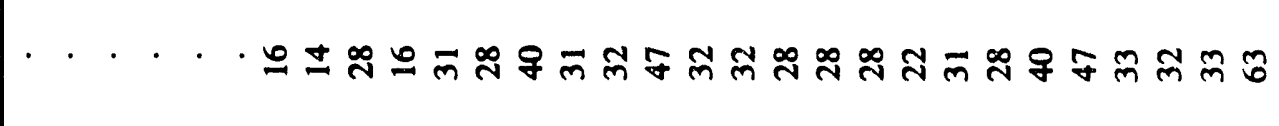 \\
\hline$\underset{n}{*}$ & 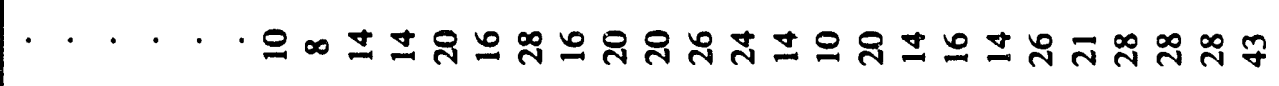 \\
\hline 票 & 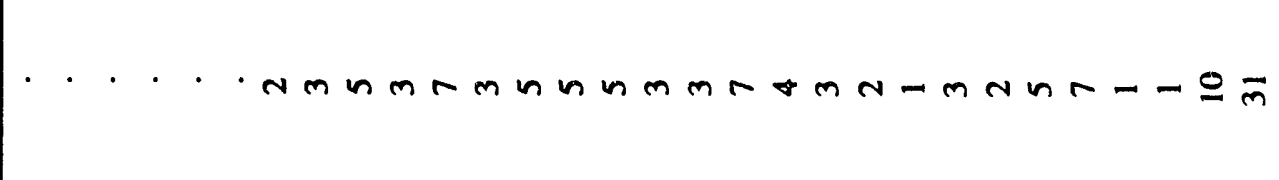 \\
\hline$\stackrel{n}{a}$ & 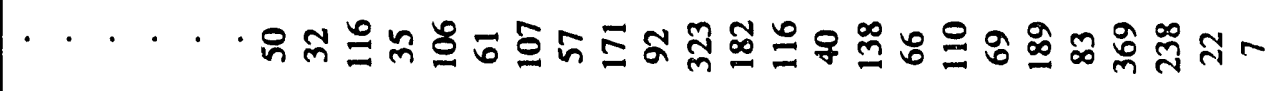 \\
\hline 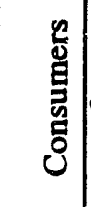 & 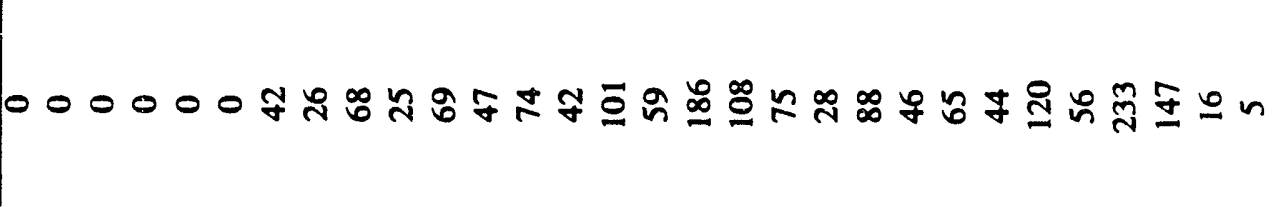 \\
\hline 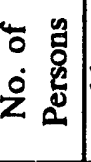 & 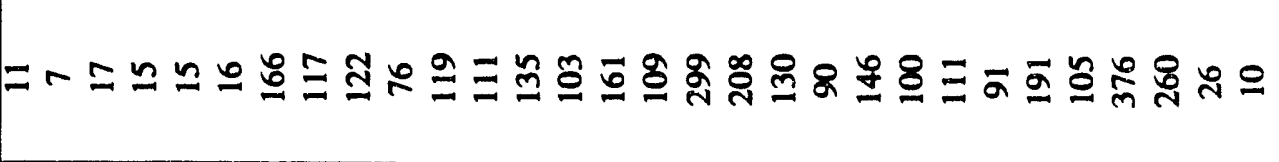 \\
\hline 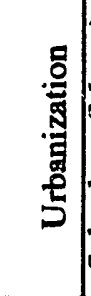 & 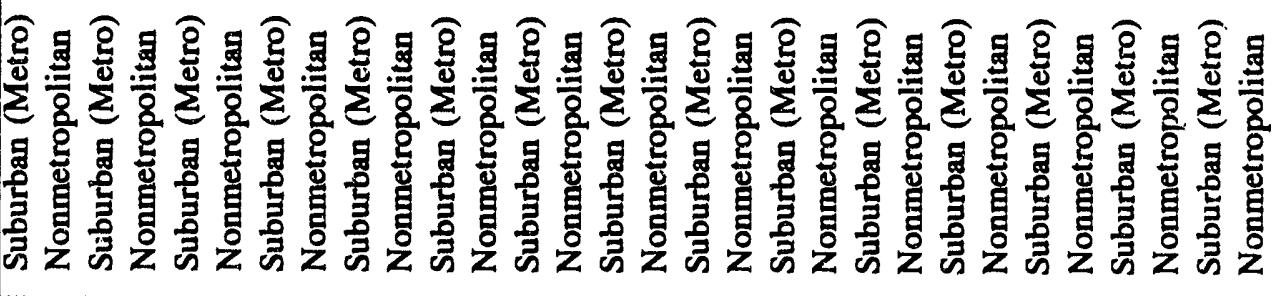 \\
\hline : & 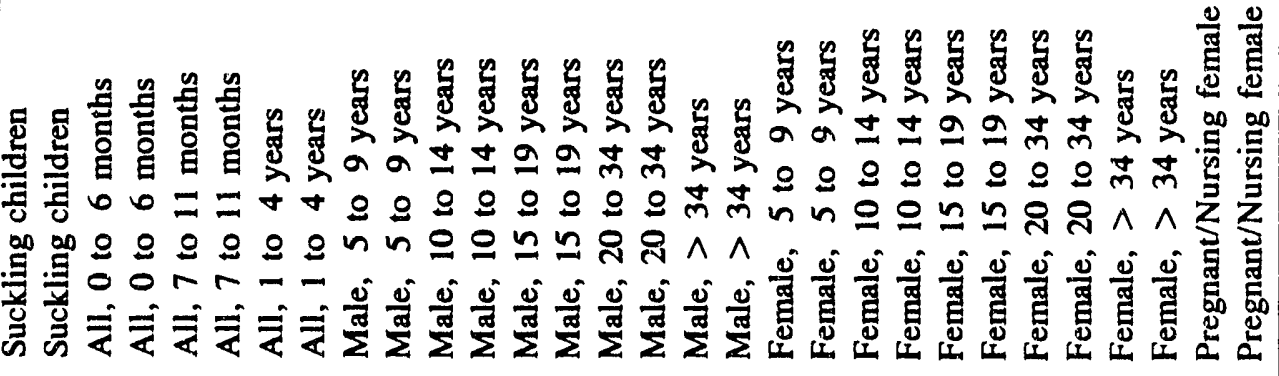 \\
\hline
\end{tabular}




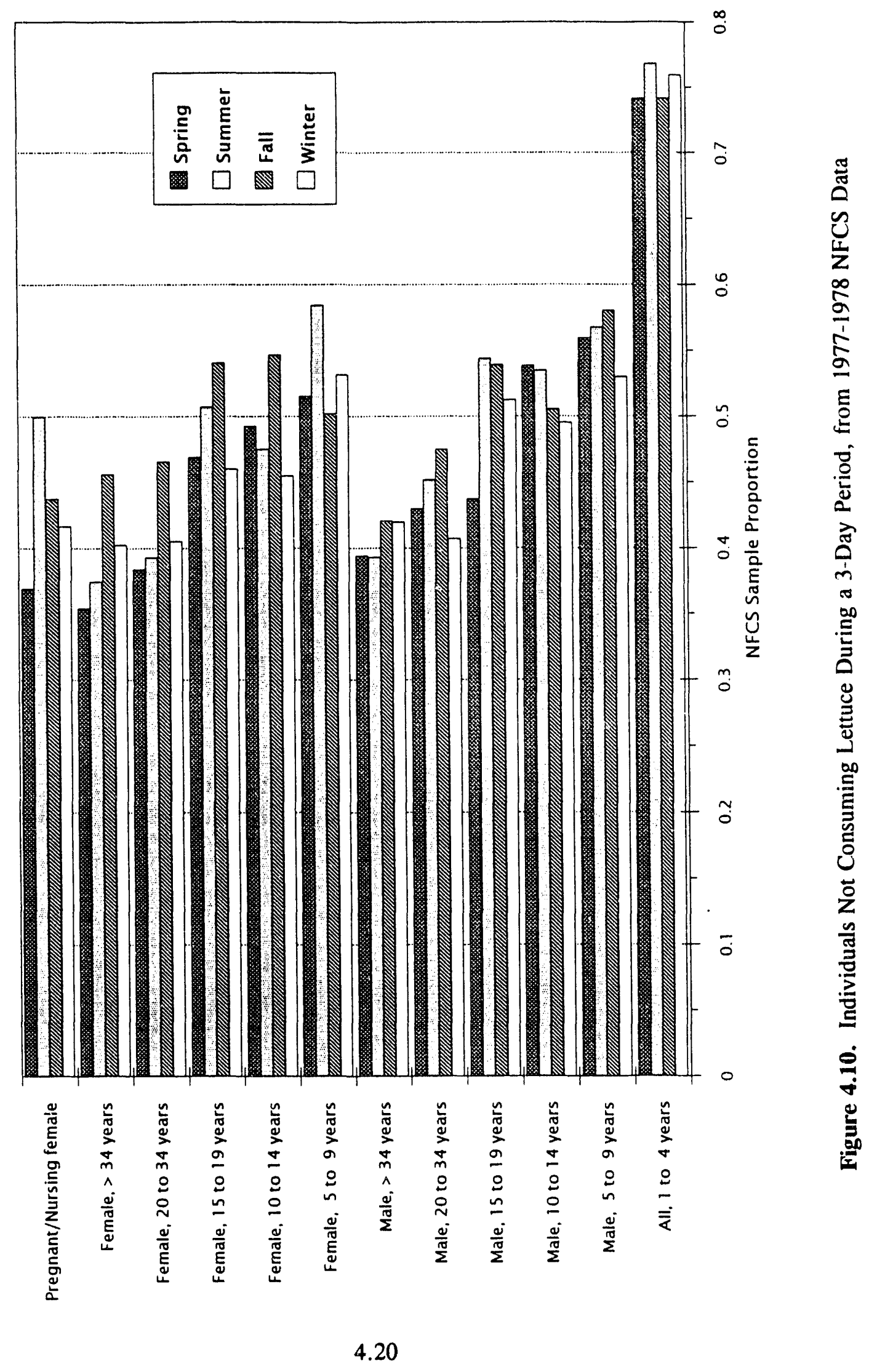




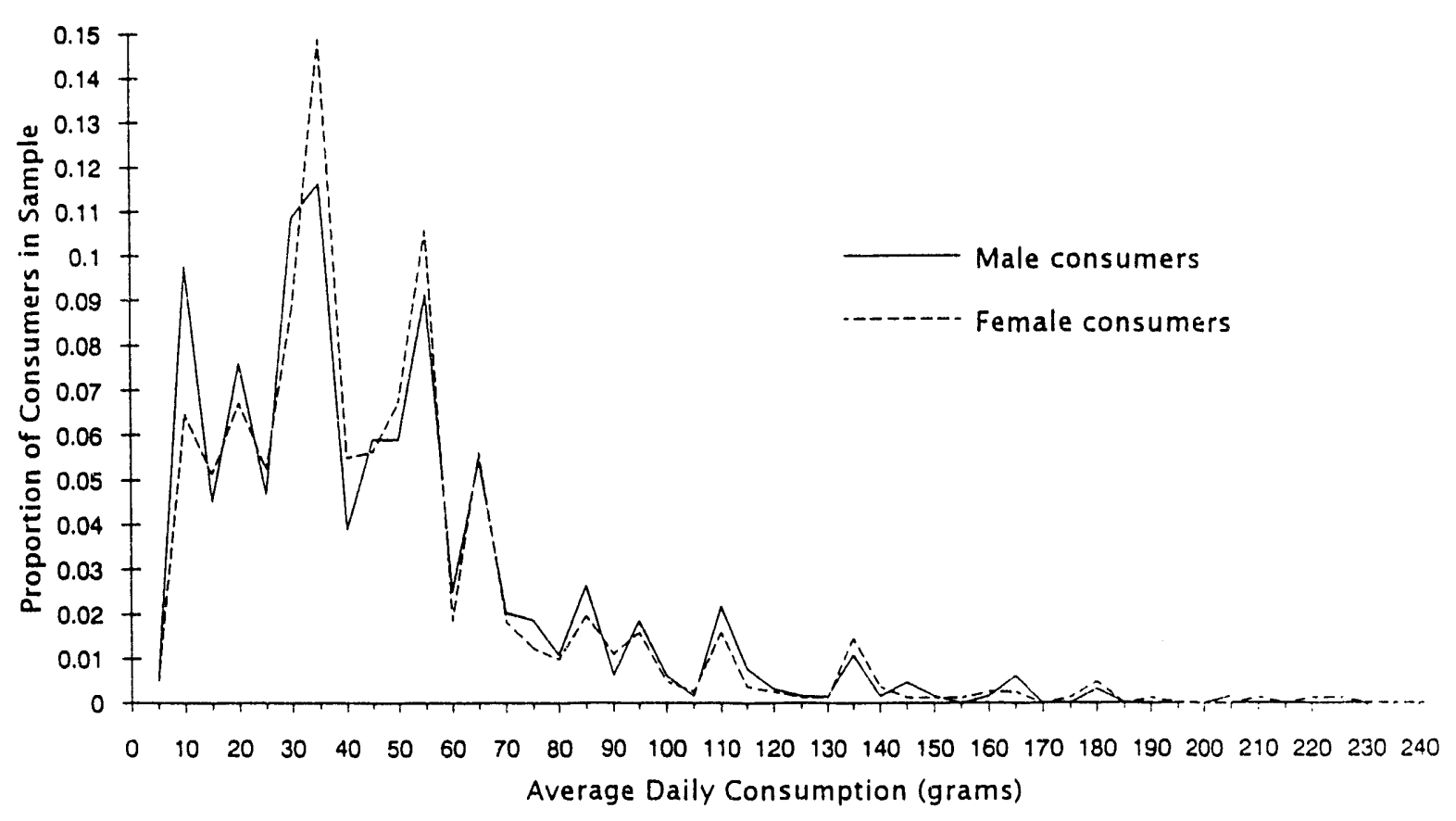

Figure 4.11. Distribution of Lettuce Consumption for Ages 20-34, from 1977-1978 NFCS Data

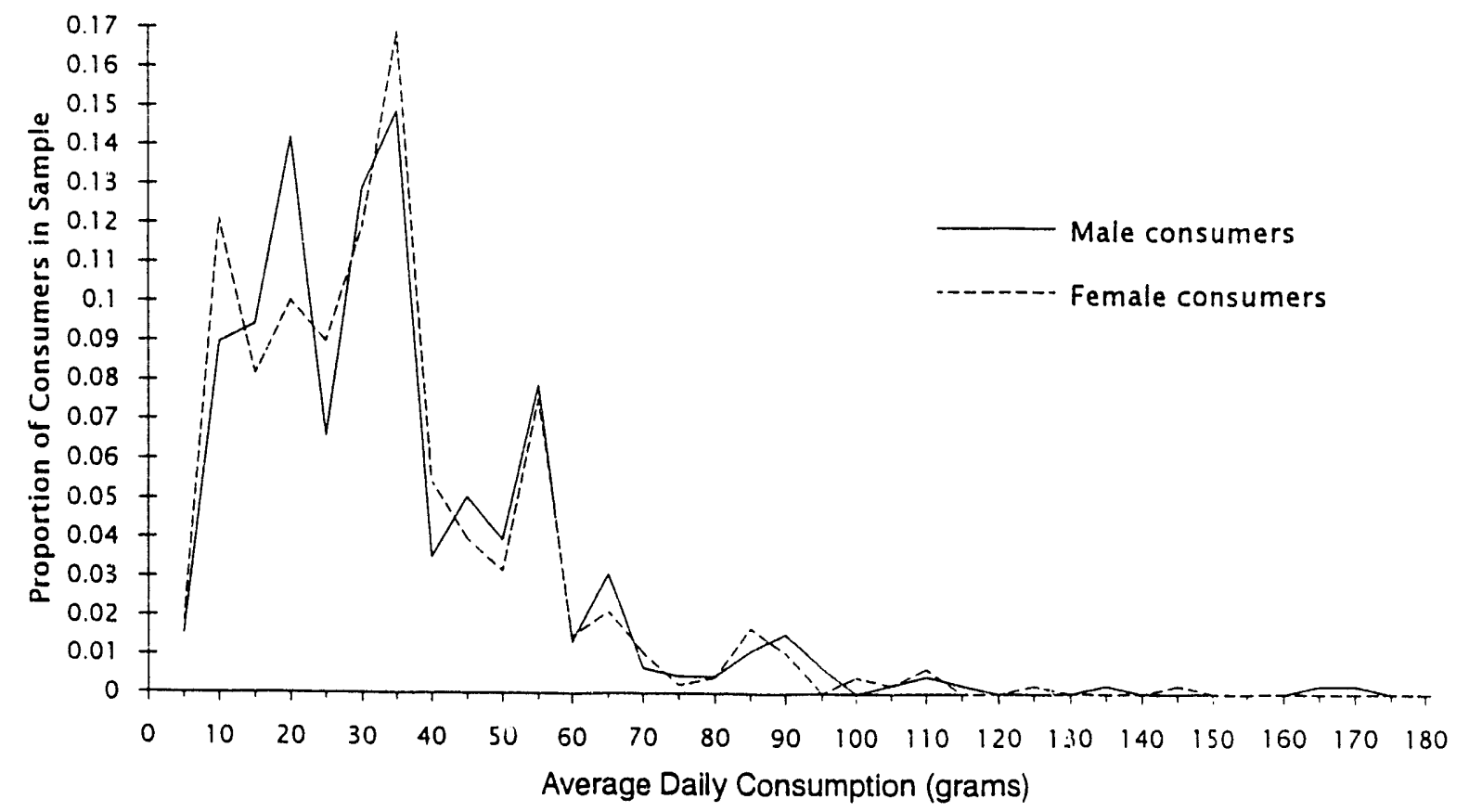

Figure 4.12. Distribution of Lettuce Consumption for Ages 10-14, from 1977-1978 NFCS Data 


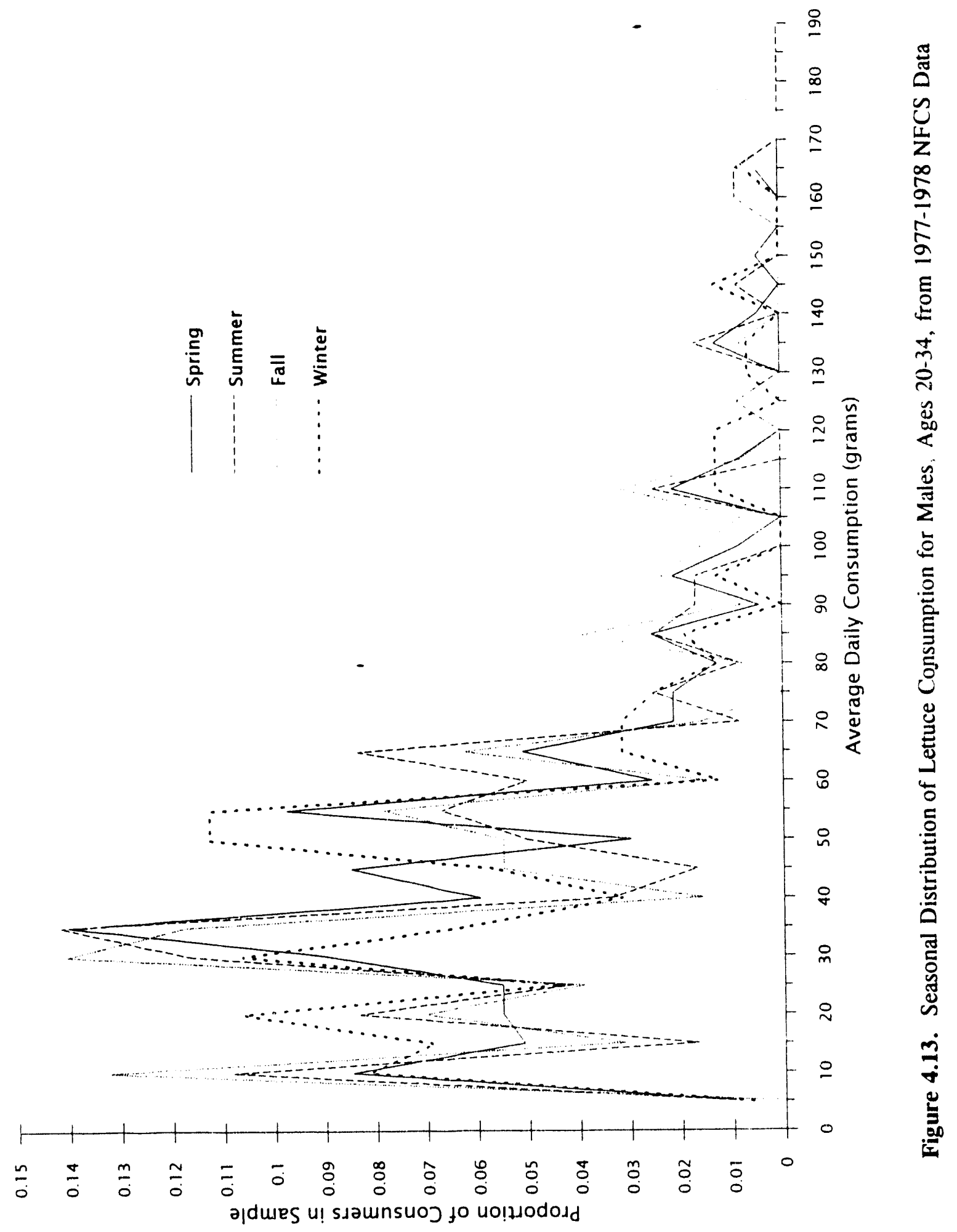




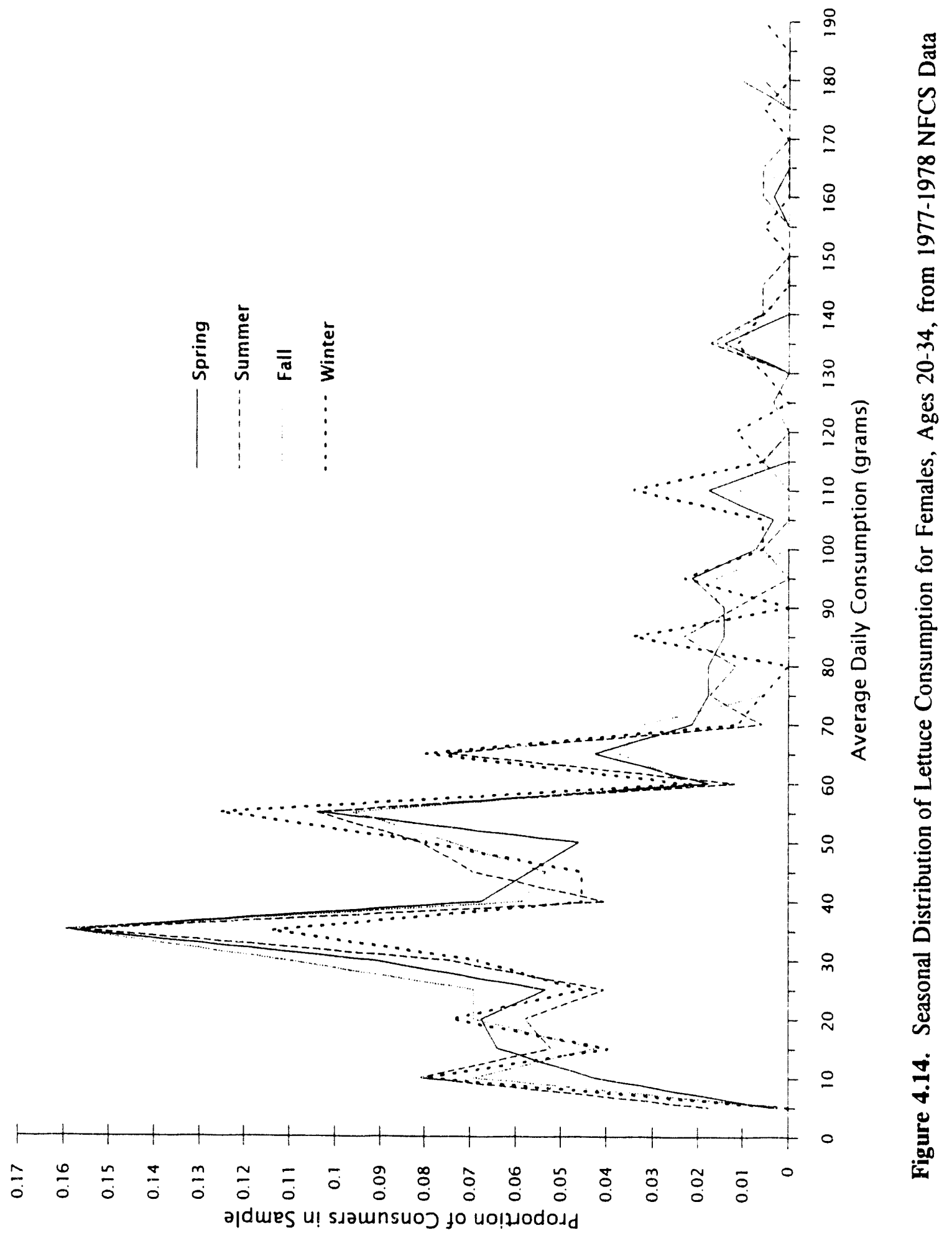




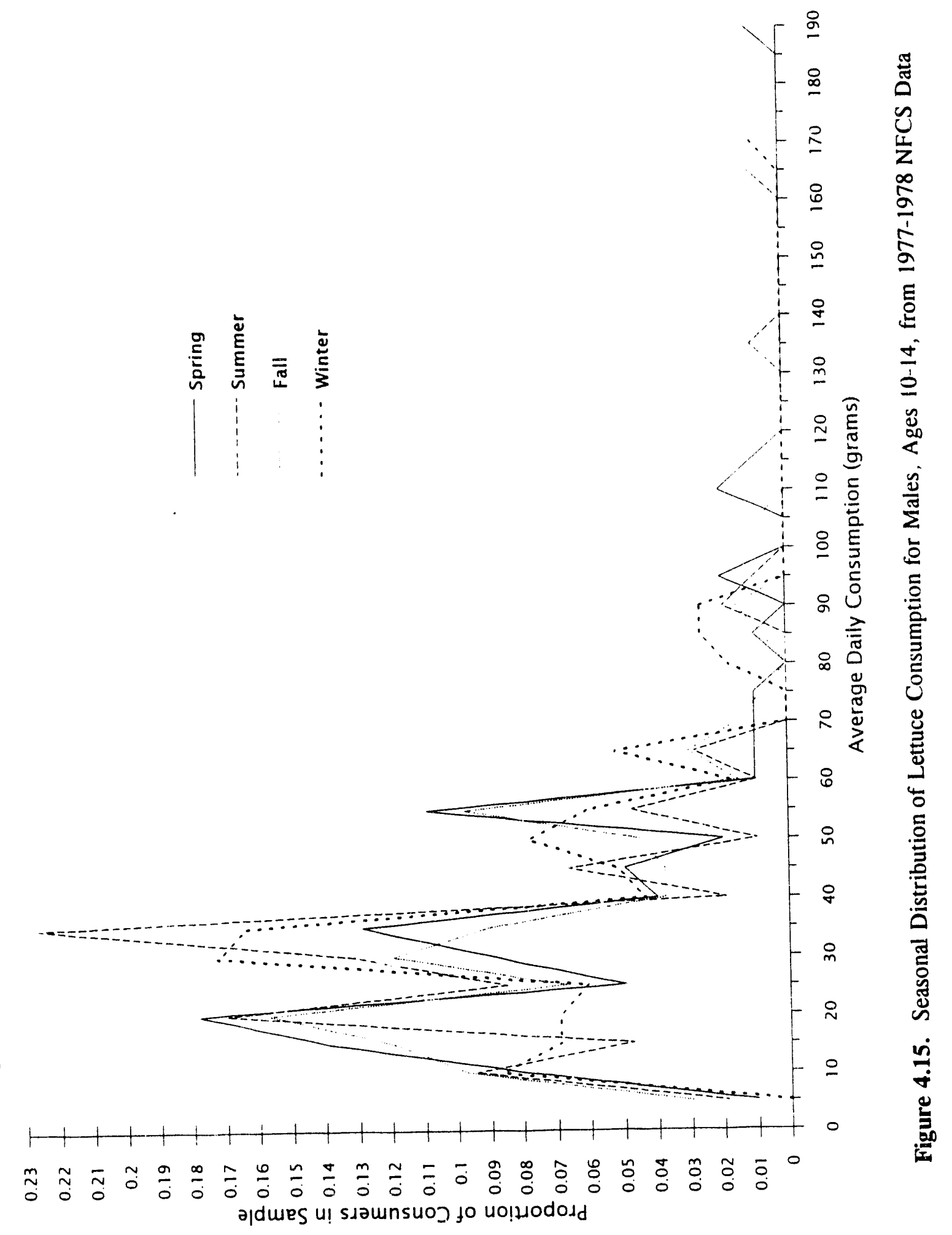




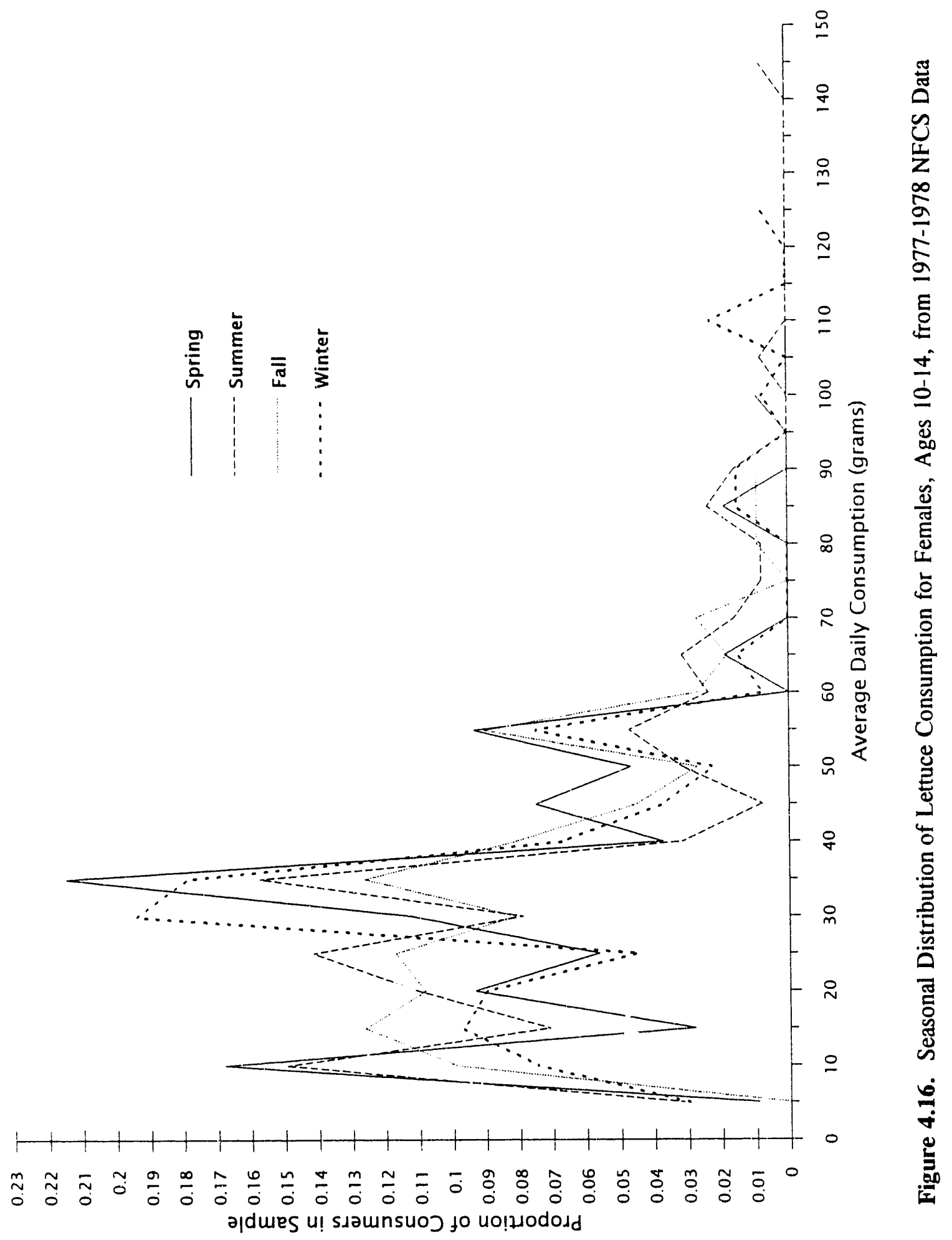




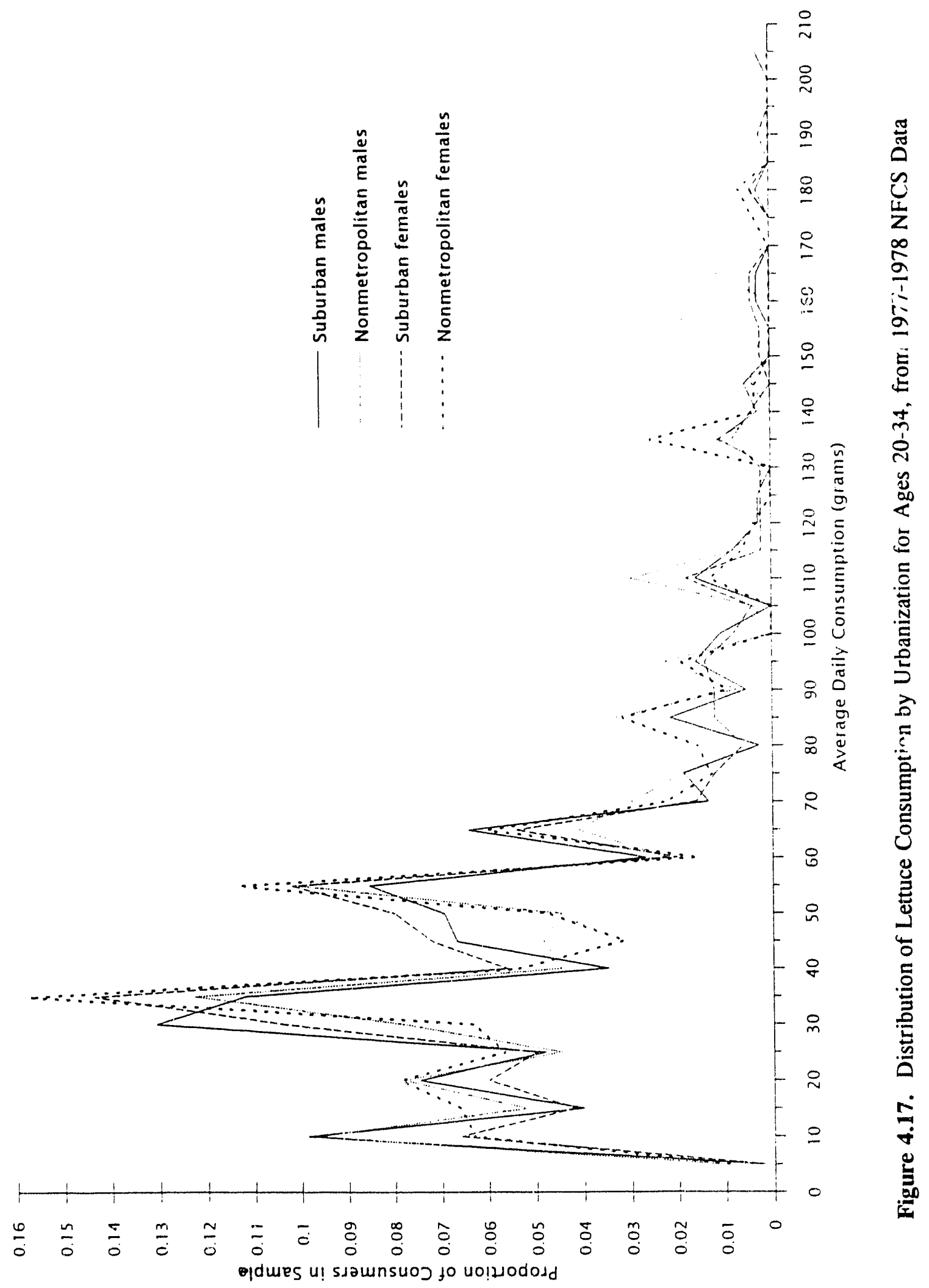




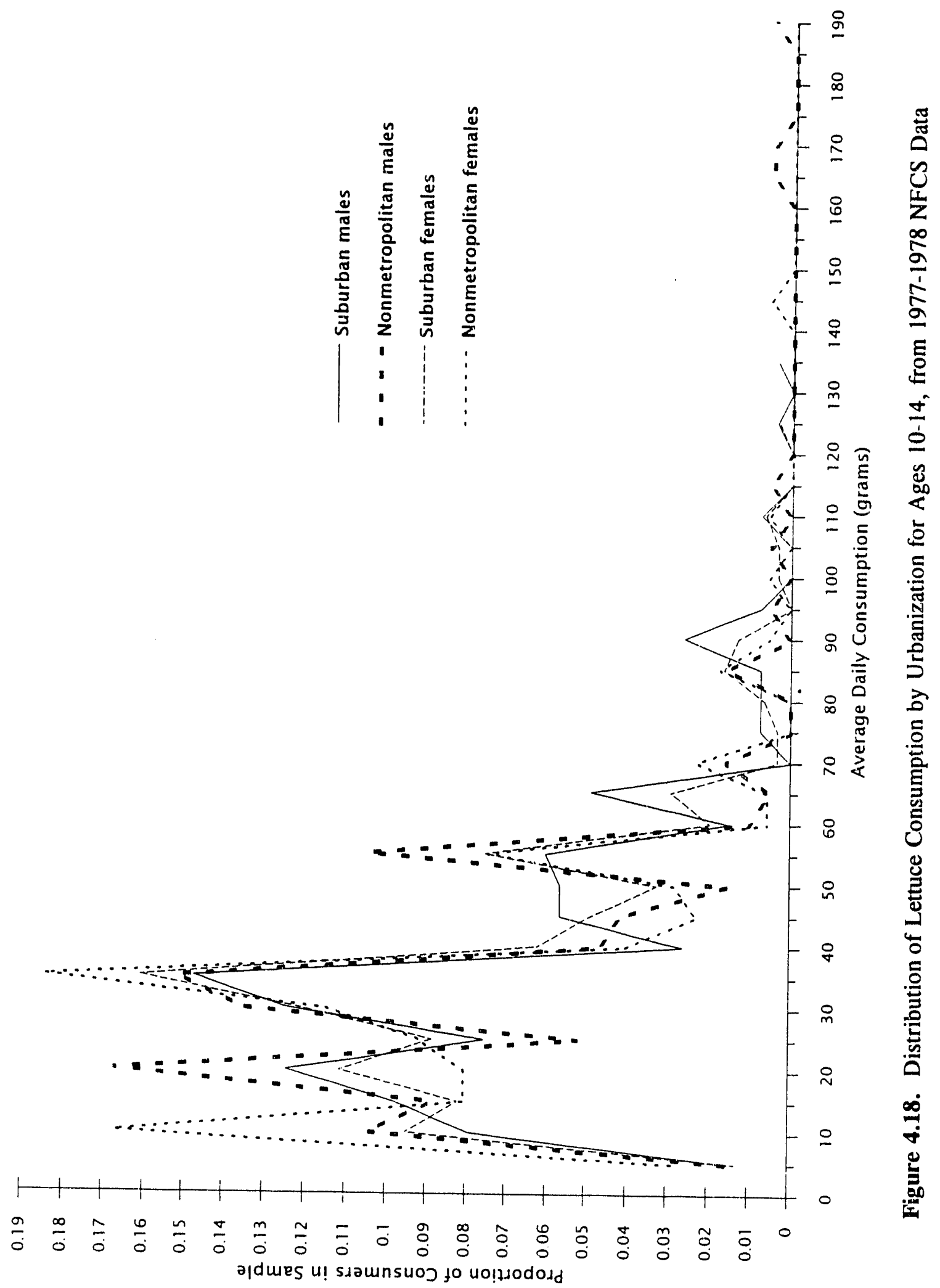




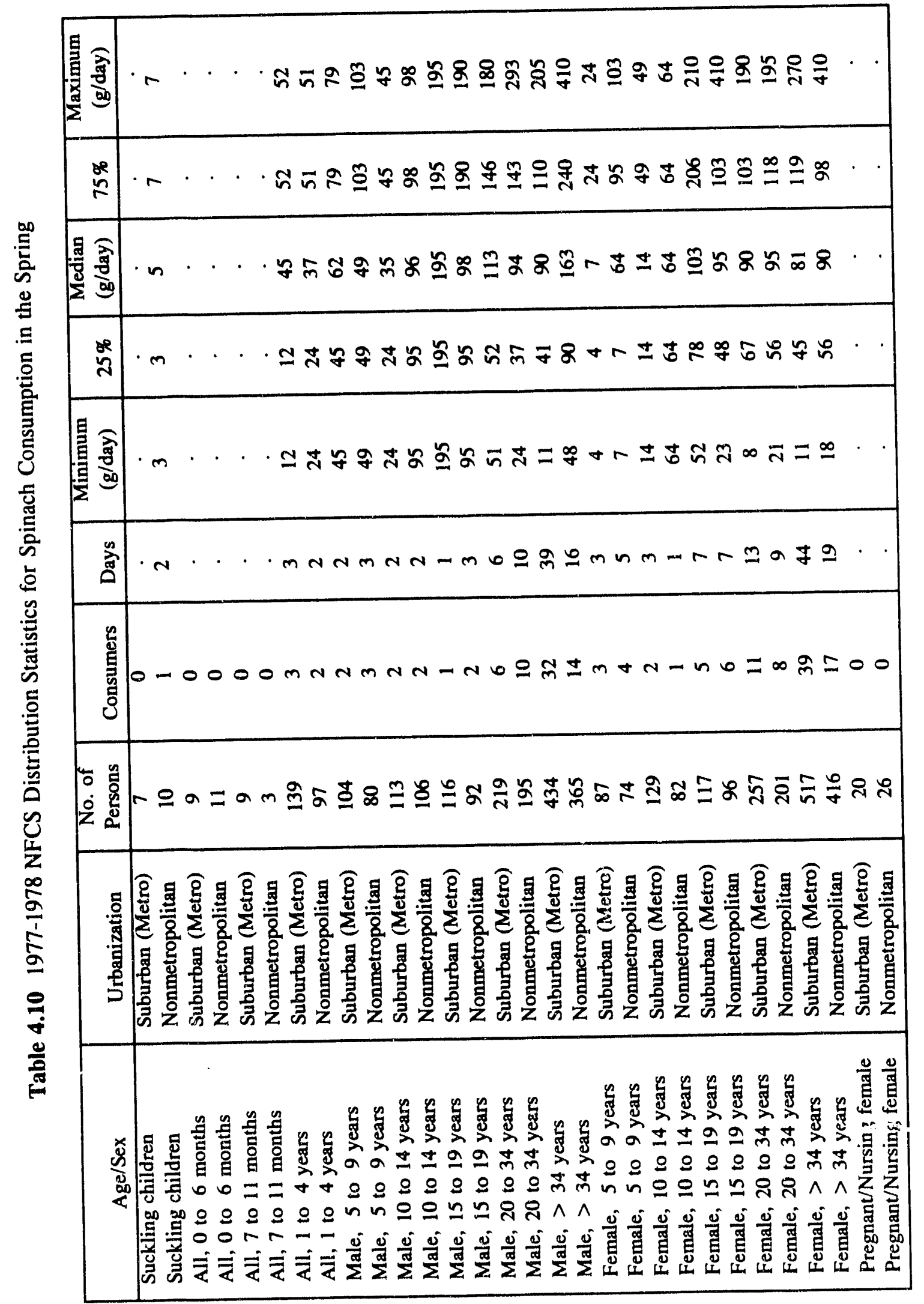




\begin{tabular}{|c|c|}
\hline 屋 & 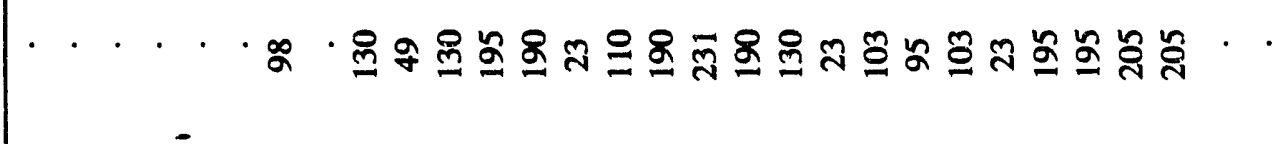 \\
\hline$\stackrel{80}{2}$ & 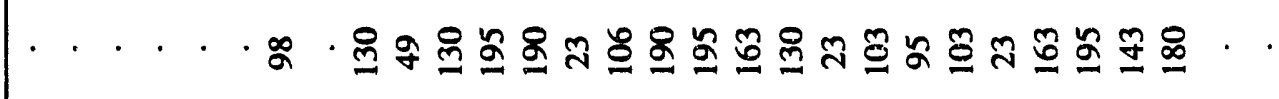 \\
\hline 焉勇 & 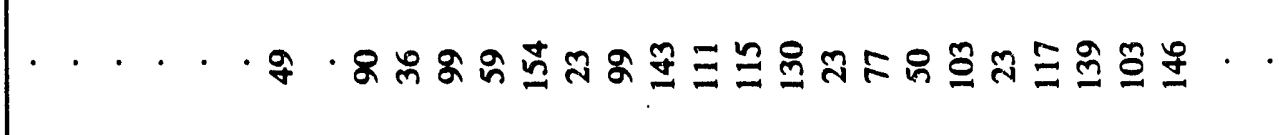 \\
\hline se & 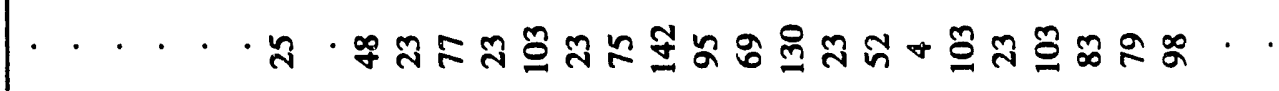 \\
\hline 通 & 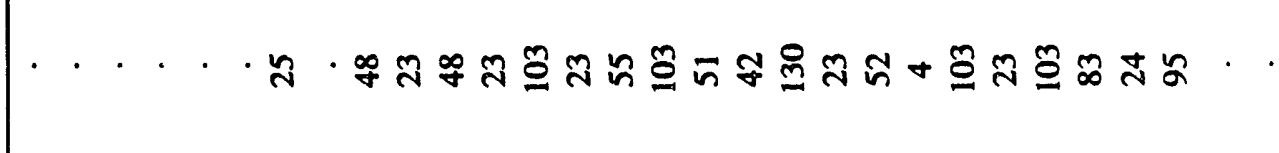 \\
\hline 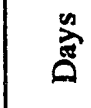 & 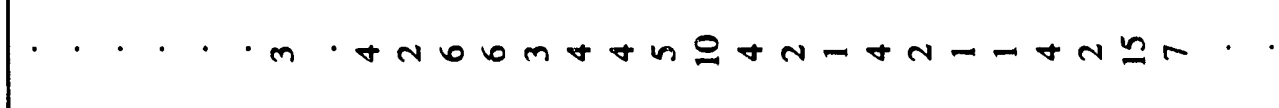 \\
\hline 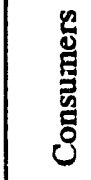 & poojoomomninontanas \\
\hline 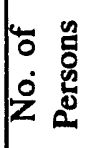 & 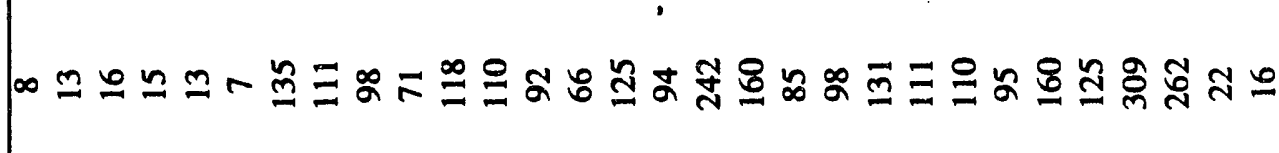 \\
\hline & 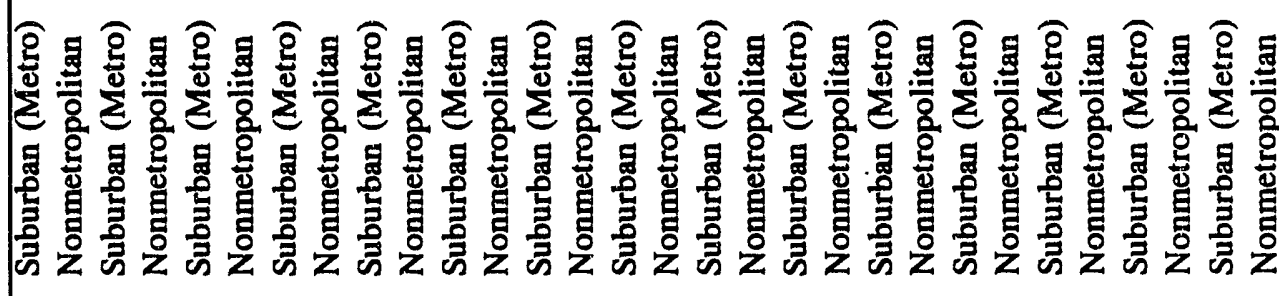 \\
\hline 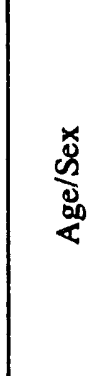 & 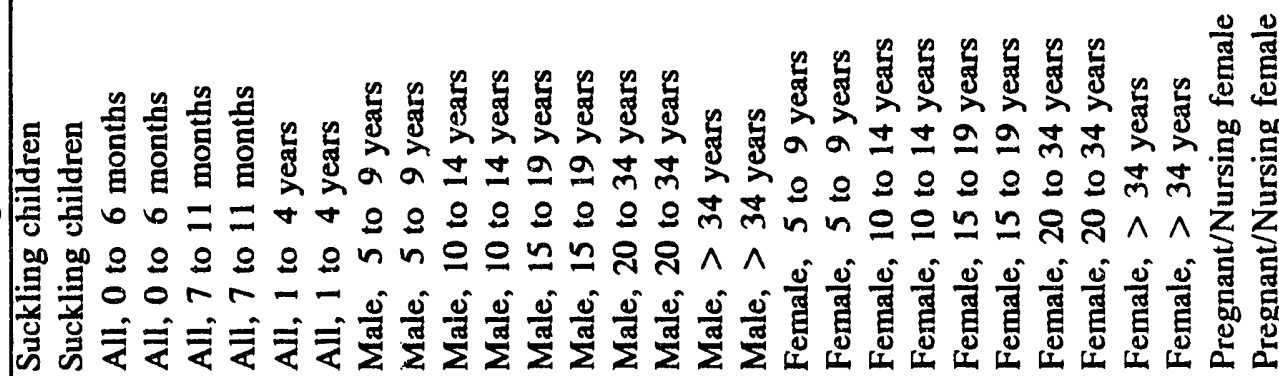 \\
\hline
\end{tabular}




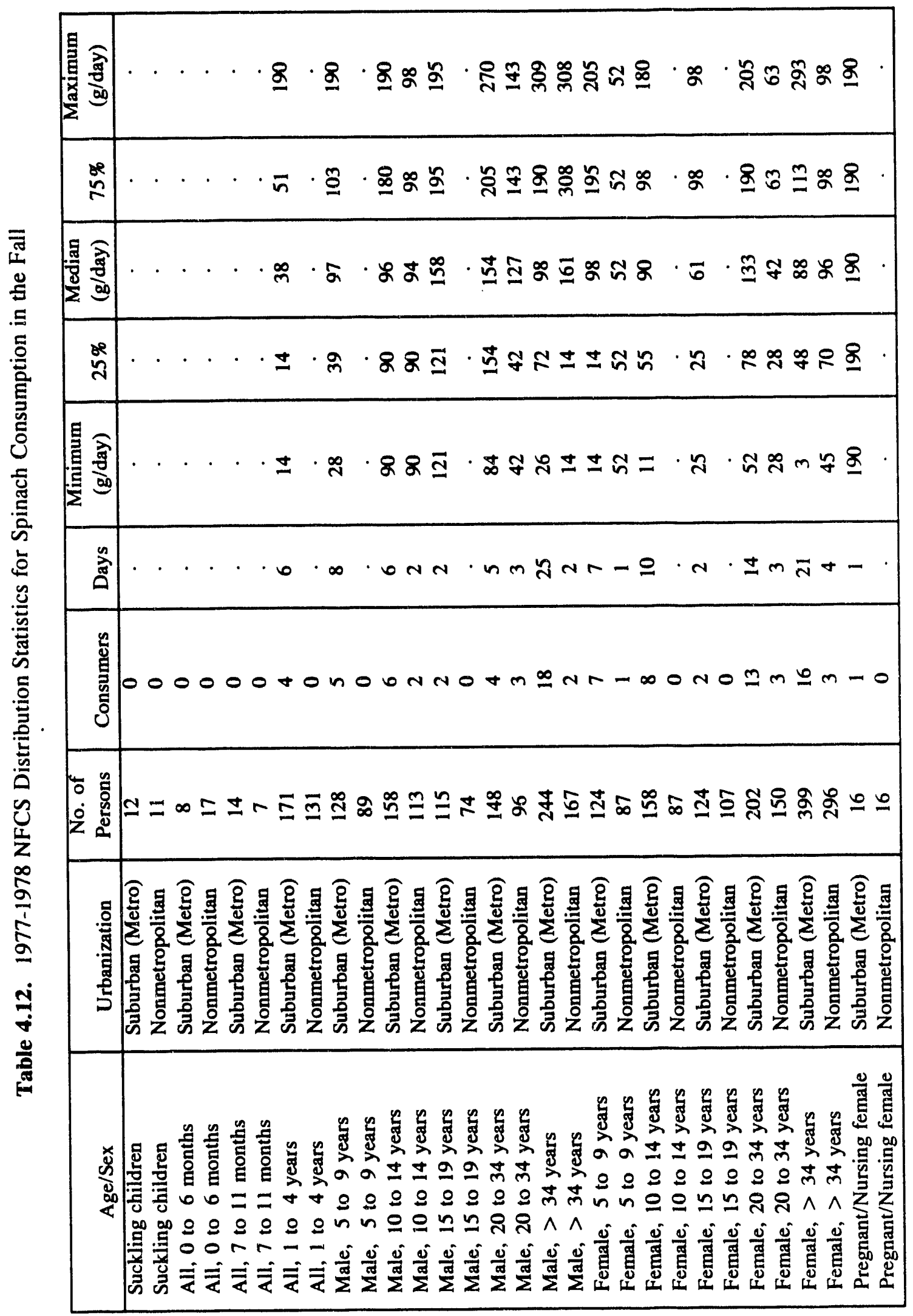




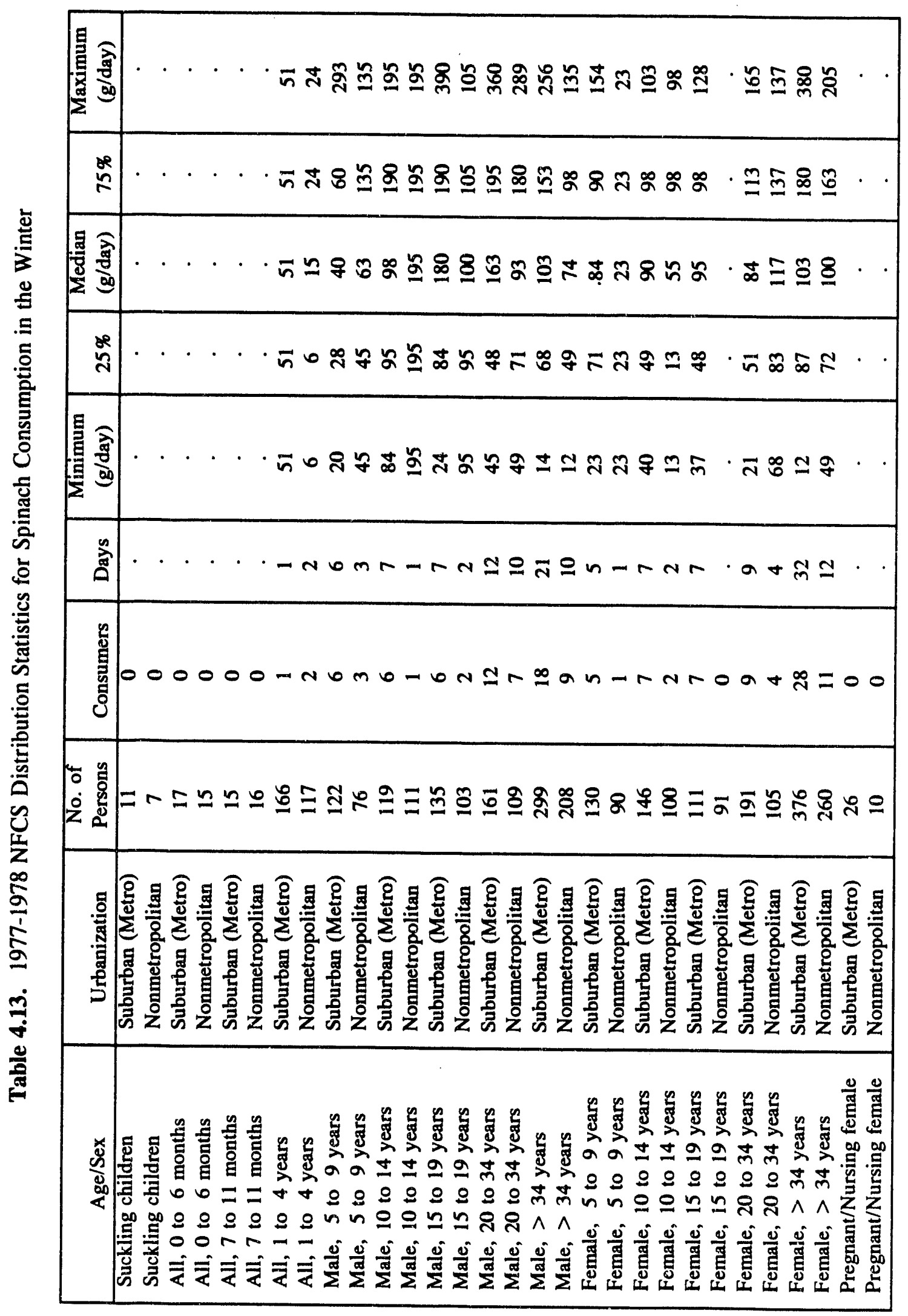




\begin{tabular}{|c|c|}
\hline 言 & 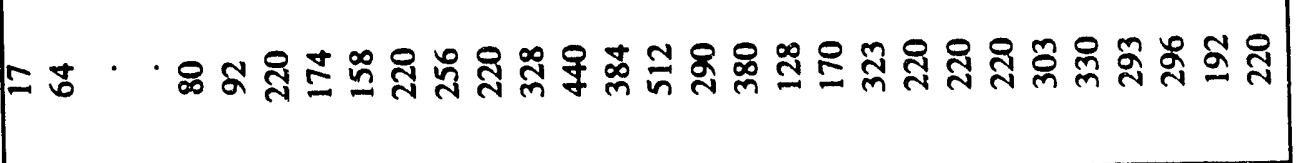 \\
\hline$\stackrel{8}{2}$ & \\
\hline 通拿 & 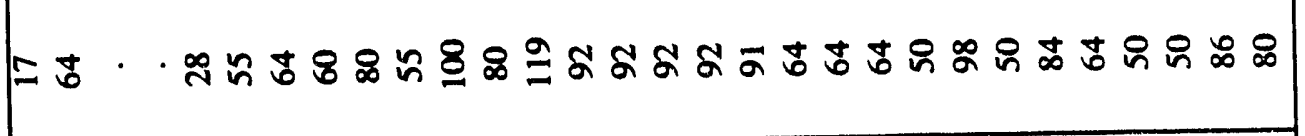 \\
\hline$\ddot{y}$ & 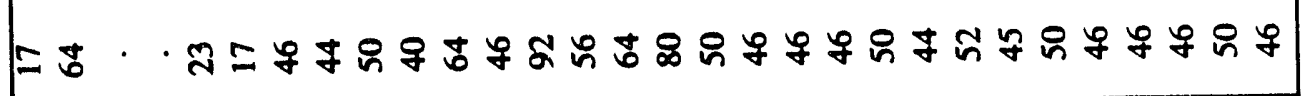 \\
\hline 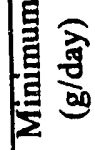 & 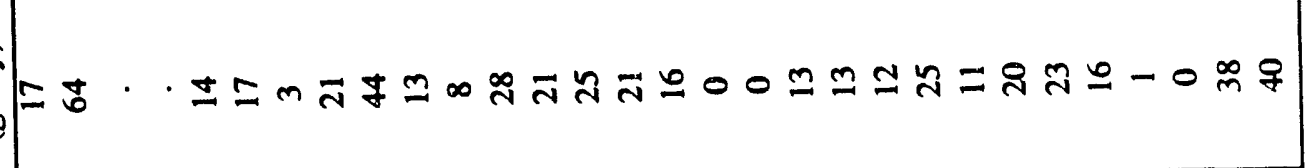 \\
\hline$\overbrace{\mathrm{A}}^{n}$ & 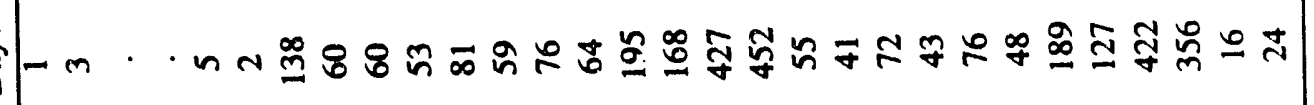 \\
\hline 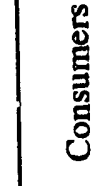 & - - \\
\hline 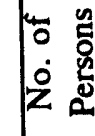 & - \\
\hline . & 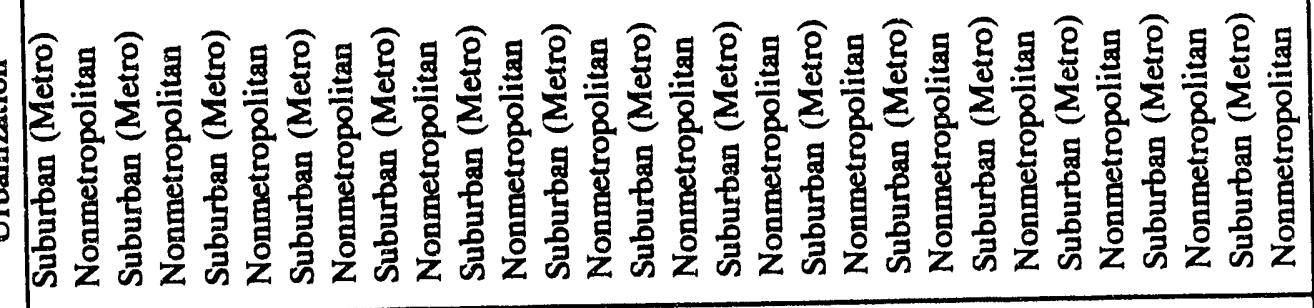 \\
\hline & 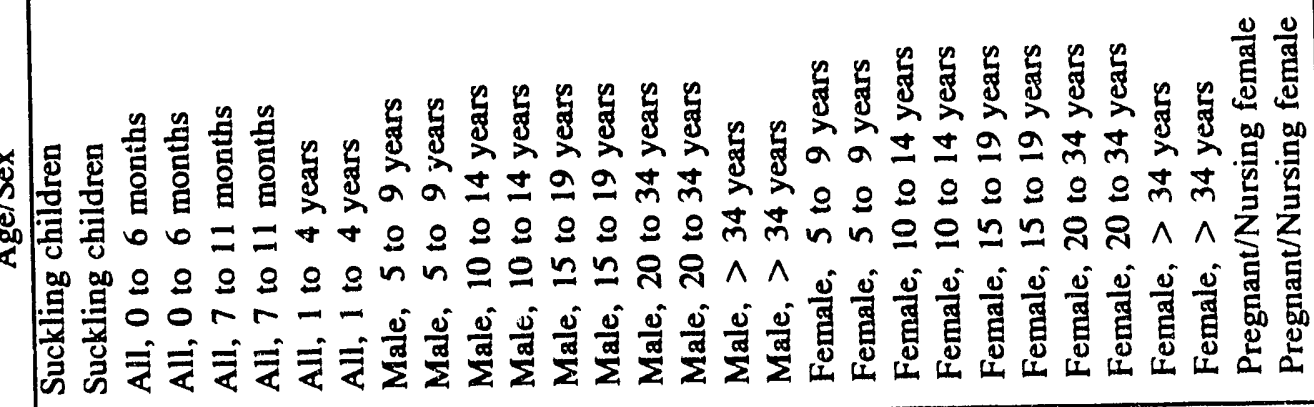 \\
\hline
\end{tabular}




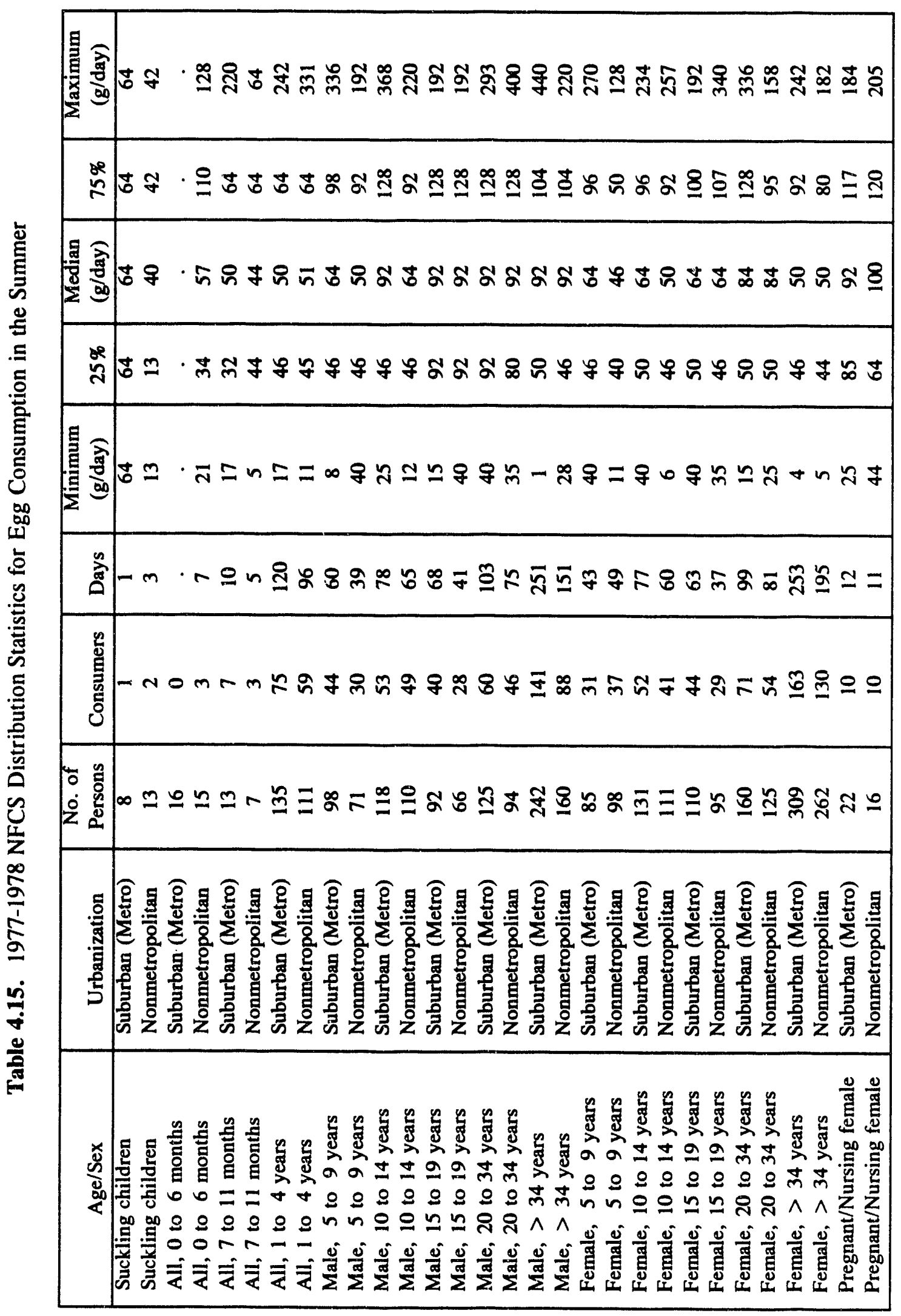




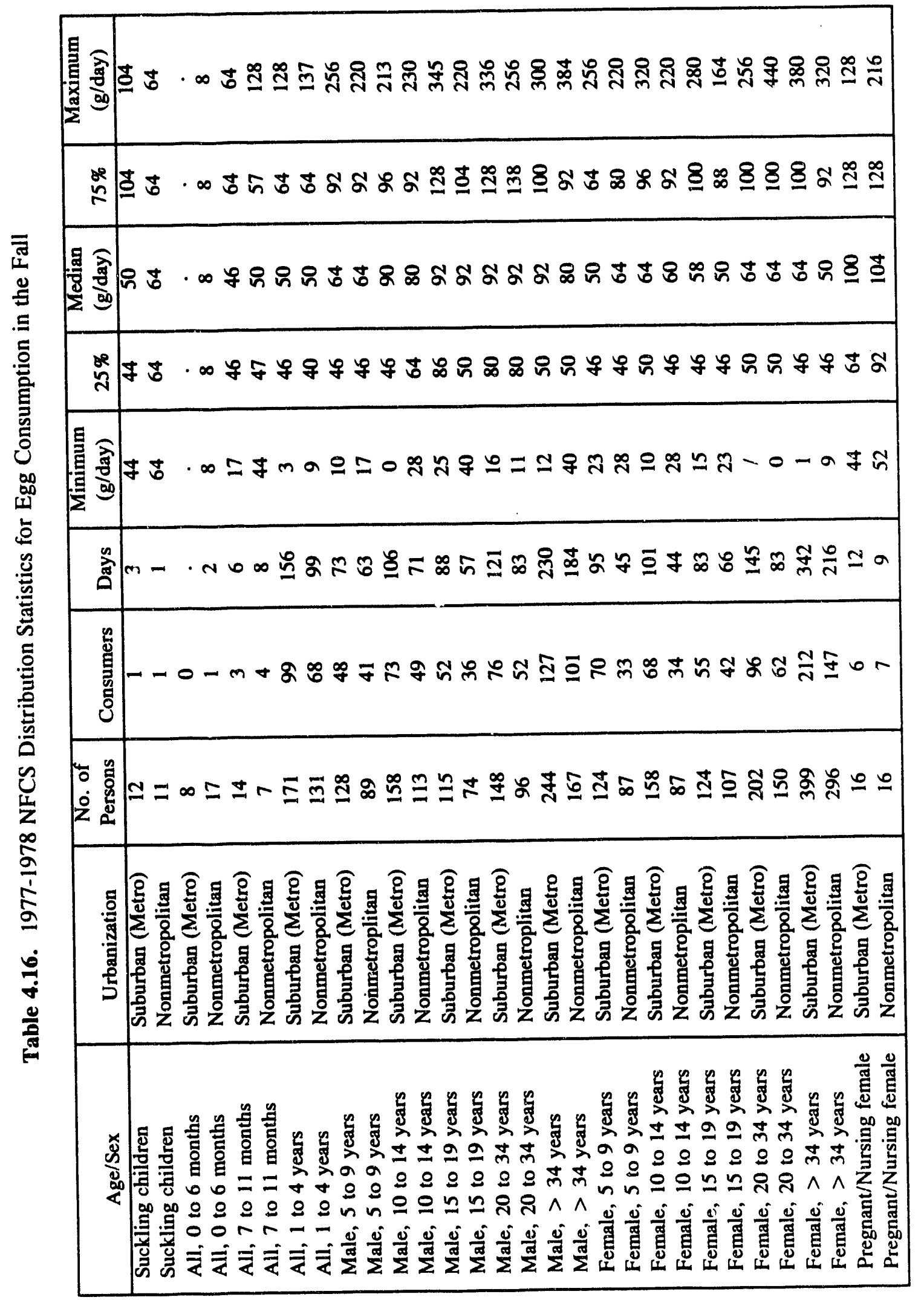




\begin{tabular}{|c|c|}
\hline 绻 & 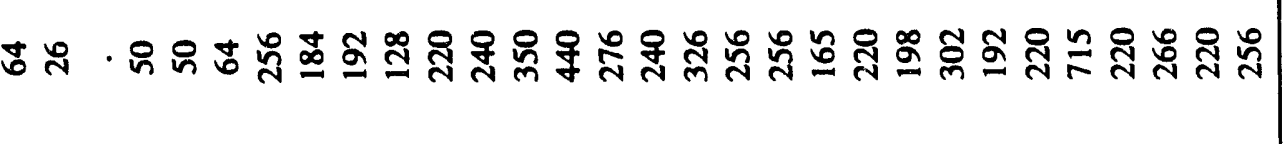 \\
\hline$\stackrel{8}{20}$ & 士 \\
\hline 焉 & 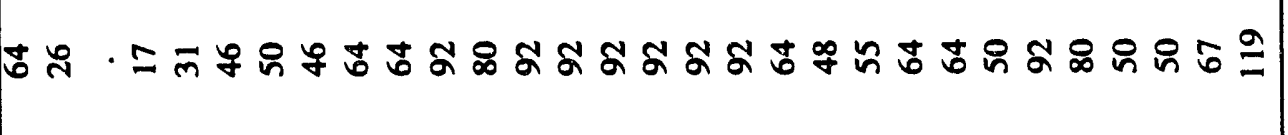 \\
\hline 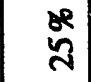 & 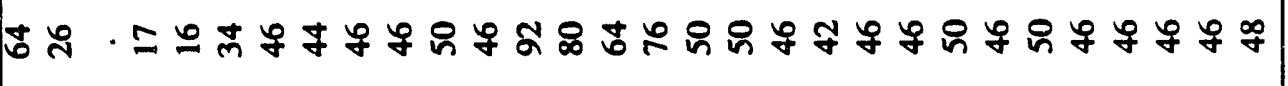 \\
\hline 鸽 & J্ \\
\hline ڤิ & ーー - \\
\hline 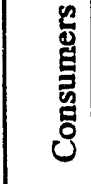 & 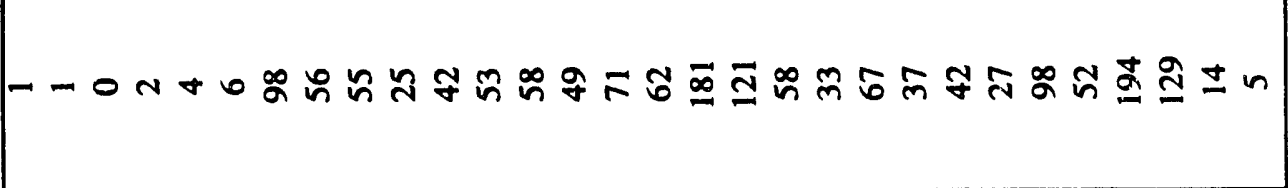 \\
\hline 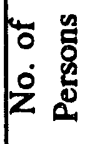 & 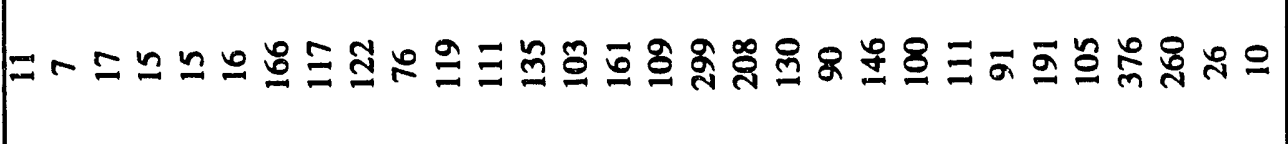 \\
\hline & 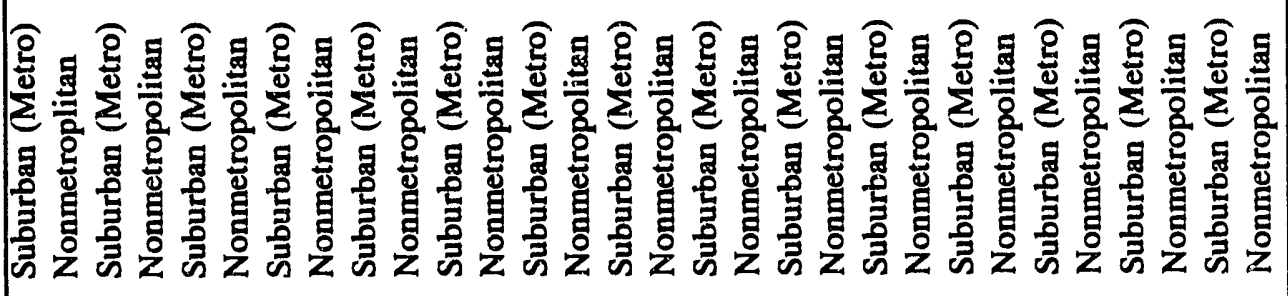 \\
\hline 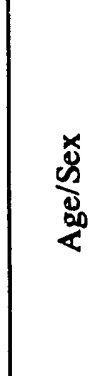 & 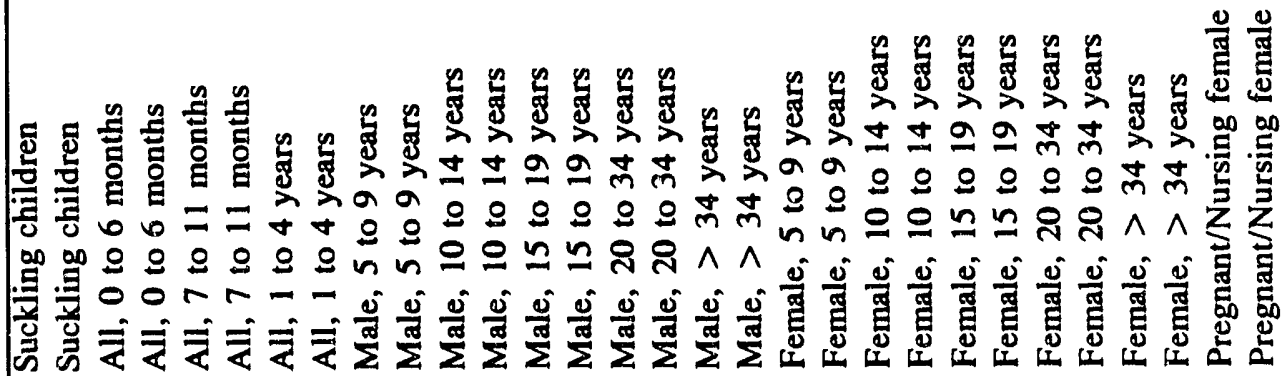 \\
\hline
\end{tabular}




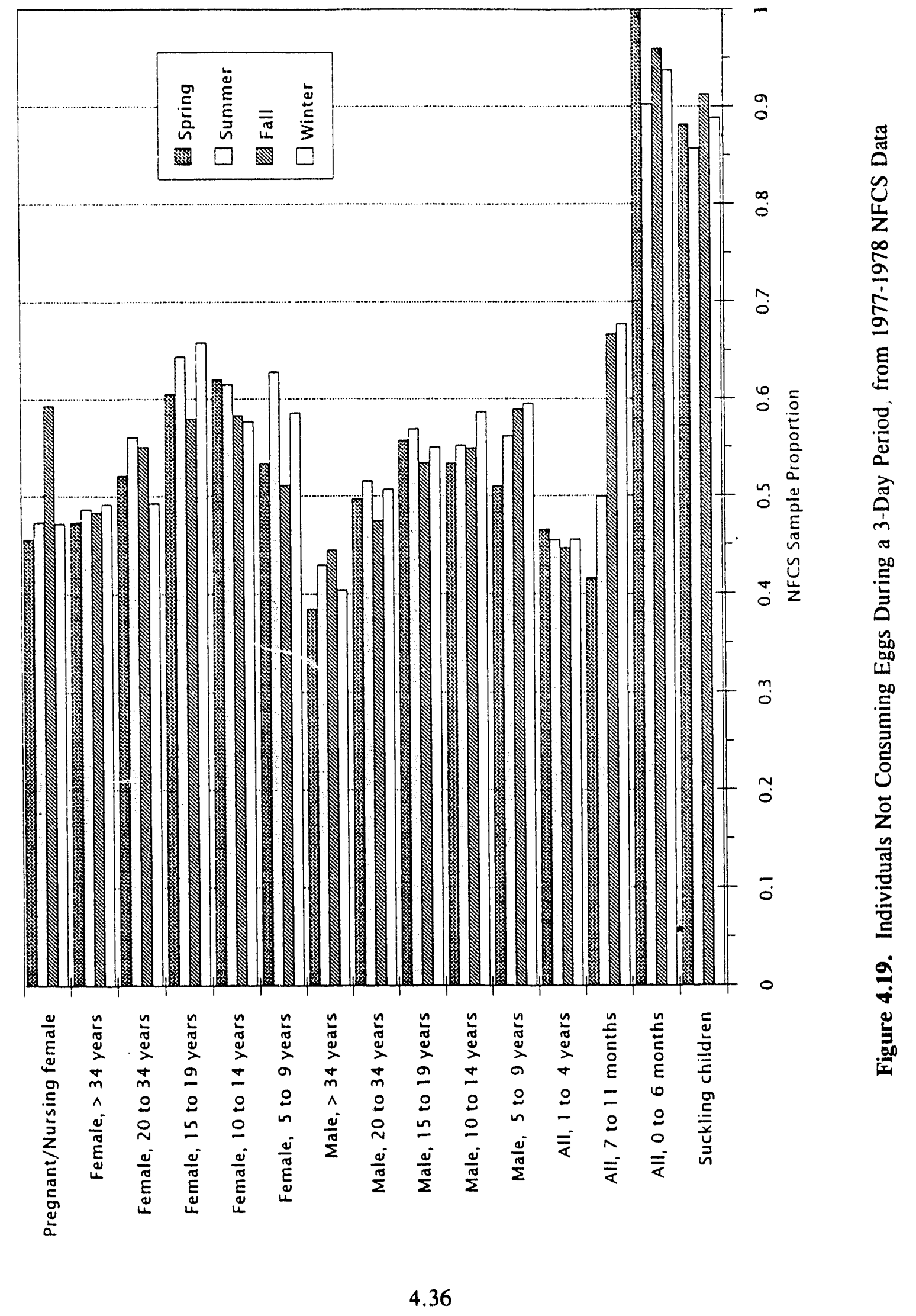




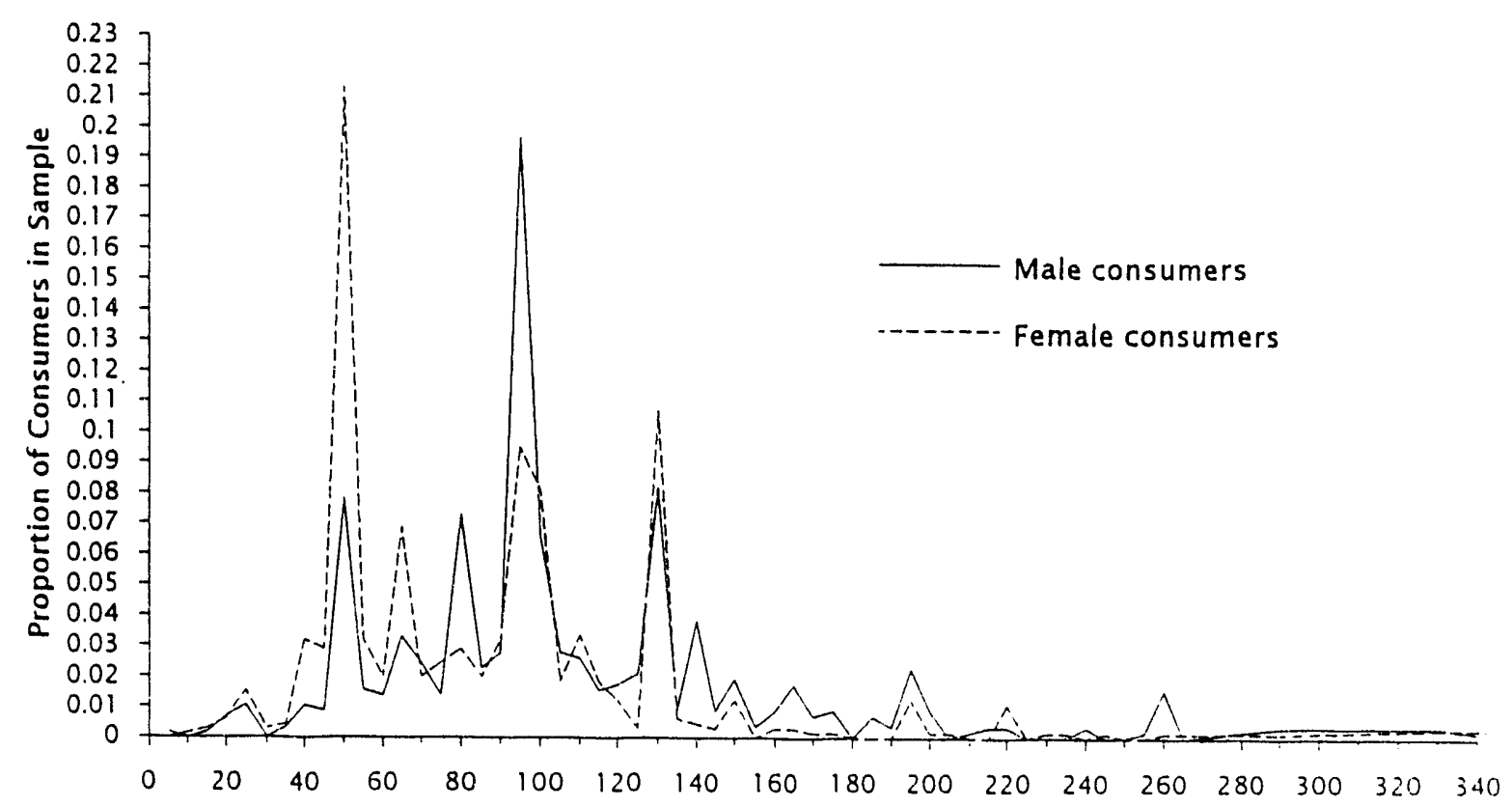

Figure 4.20. Distribution of Egg Consumption for Ages 20-34, from 1977-1978 NFCS Data

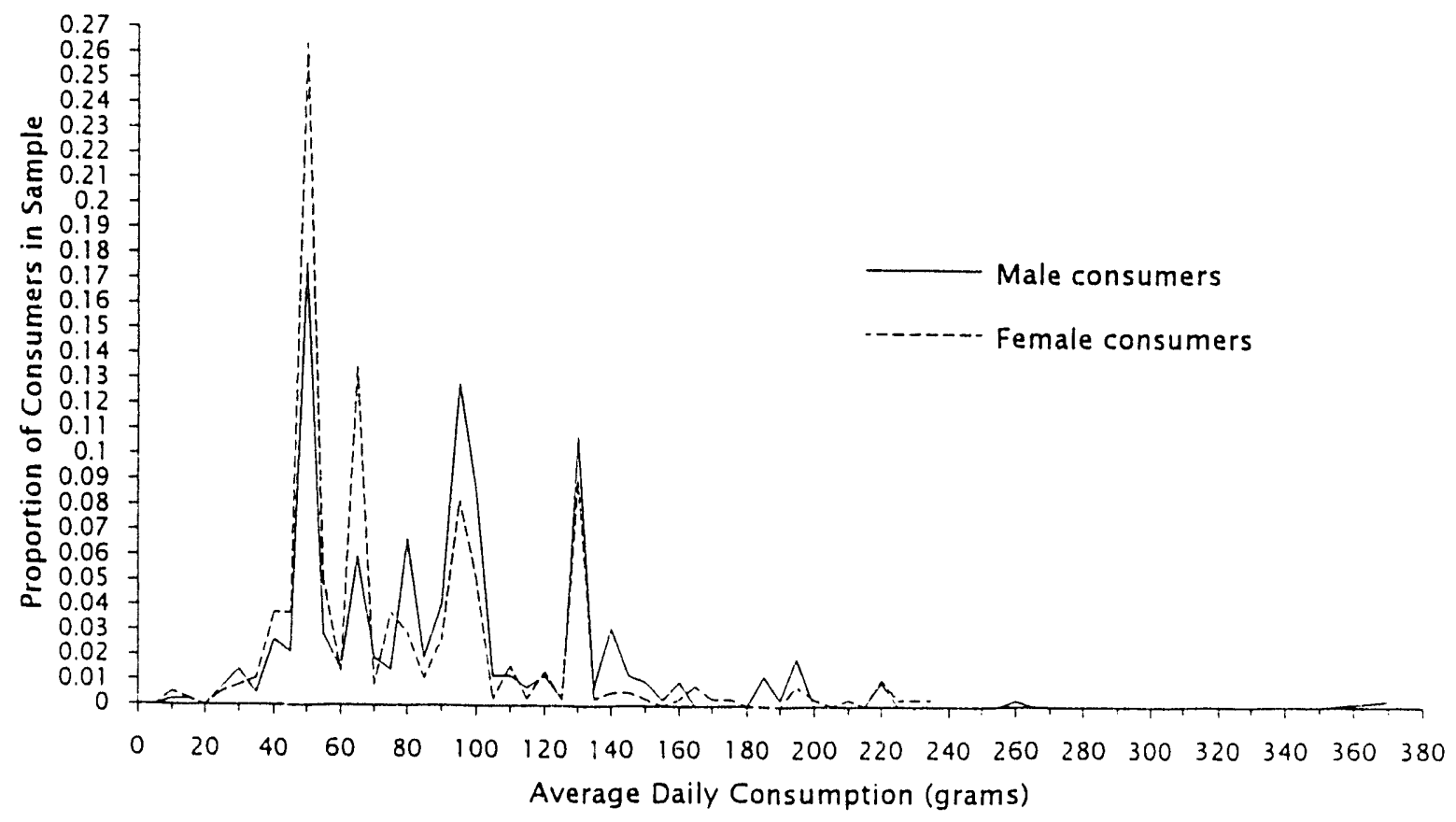

Figure 4.21. Distribution of Egg Consumption for Ages 10-14, from 1977-1978 NFCS Data 


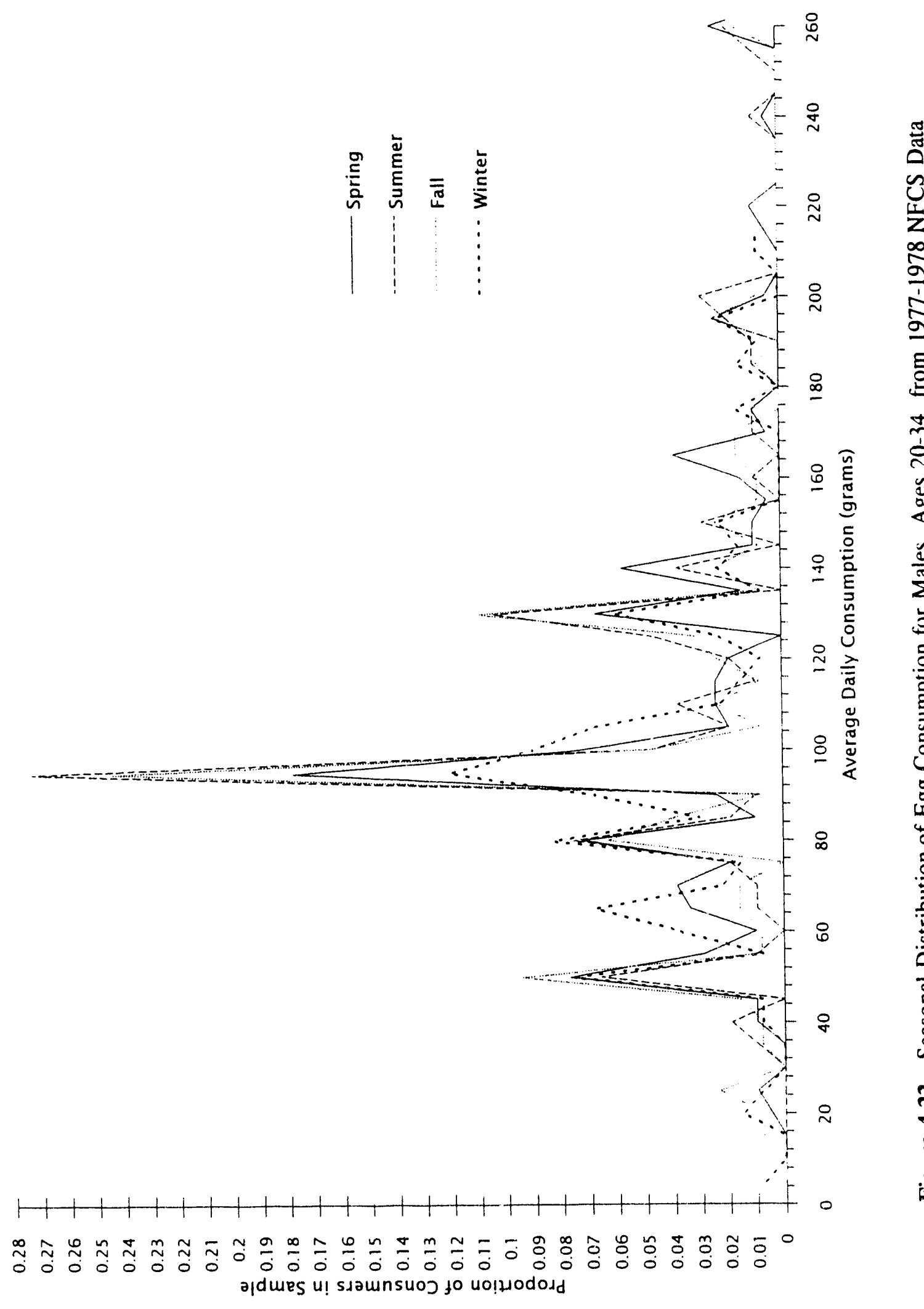

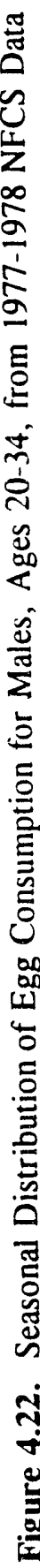




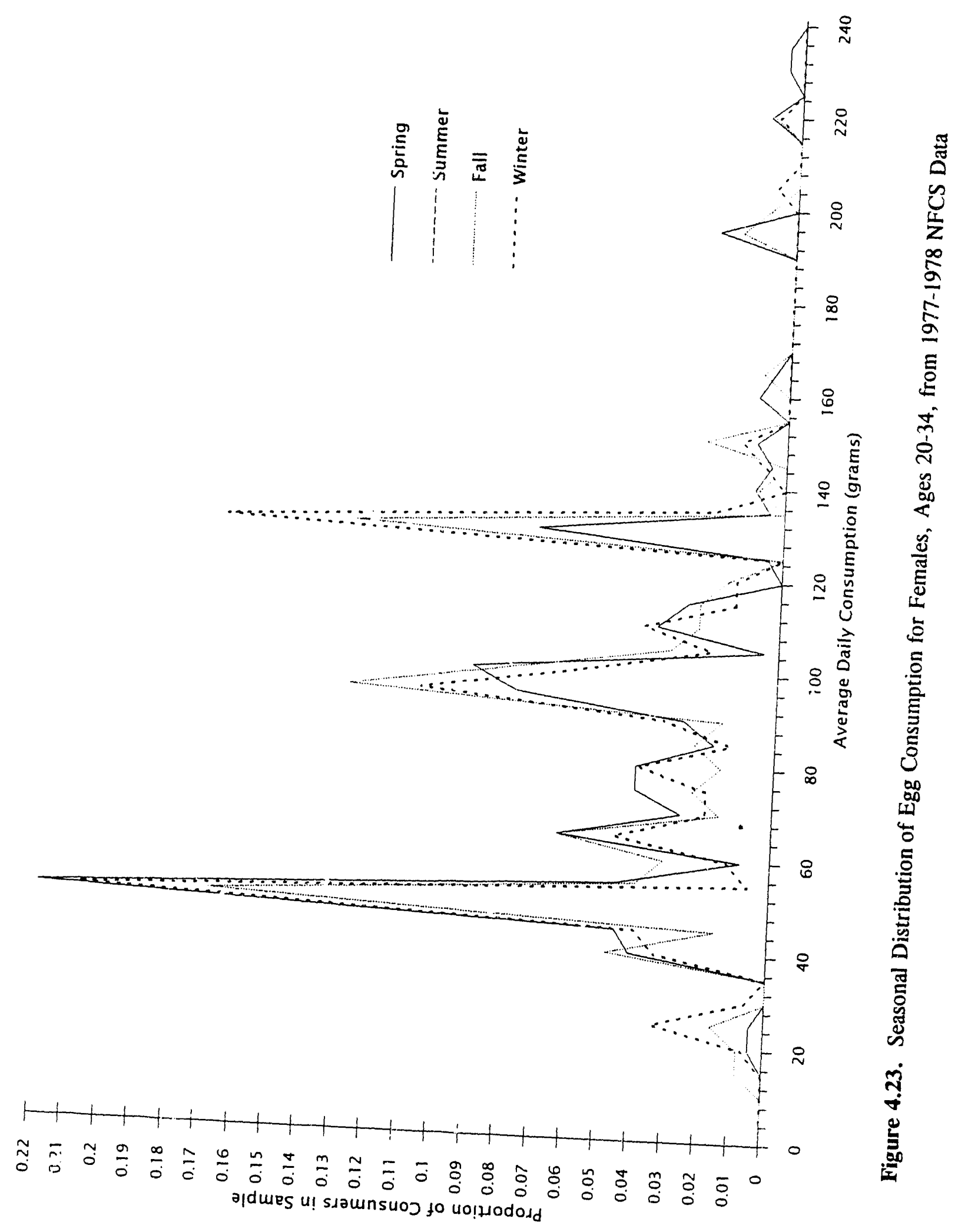




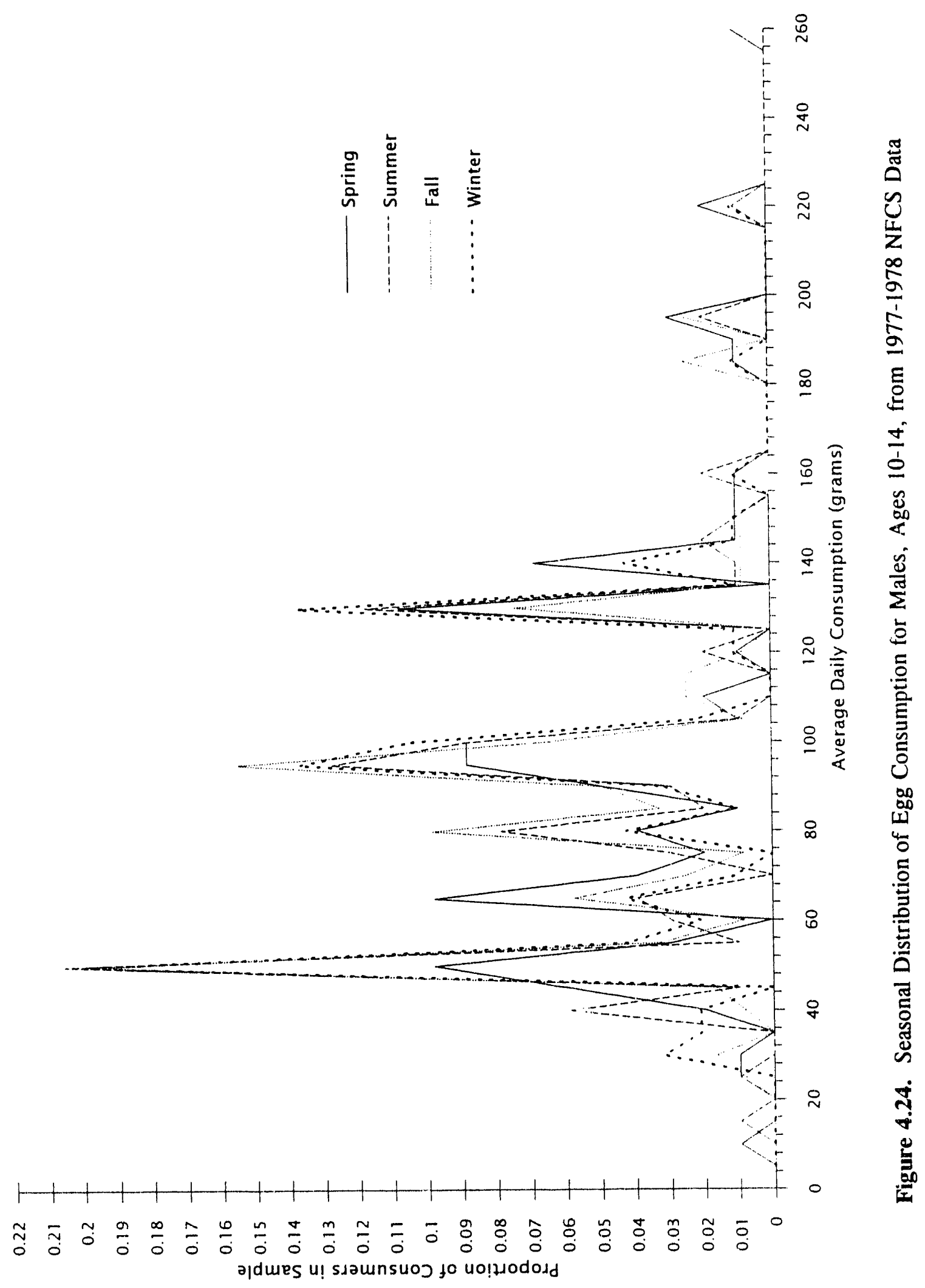




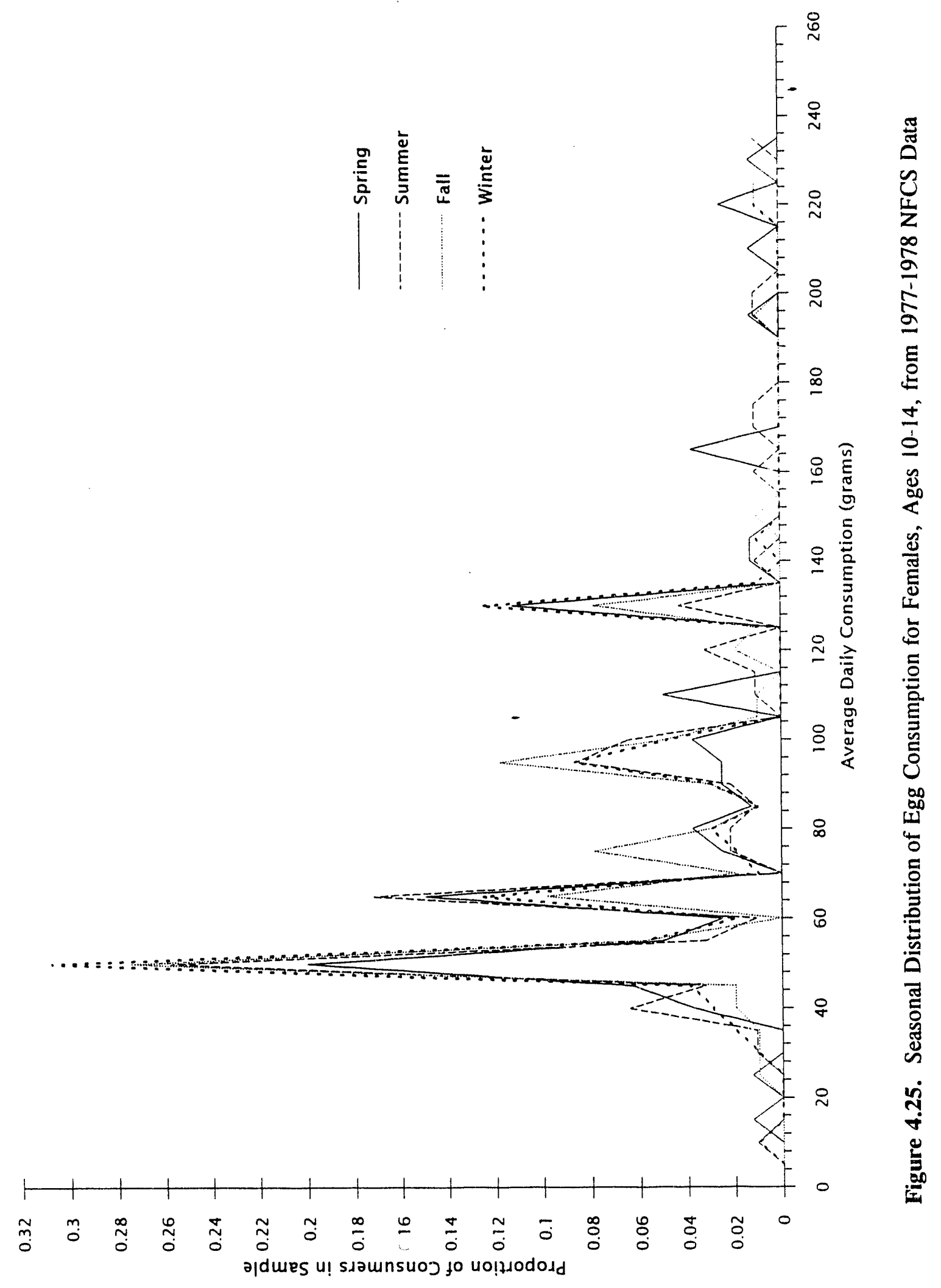




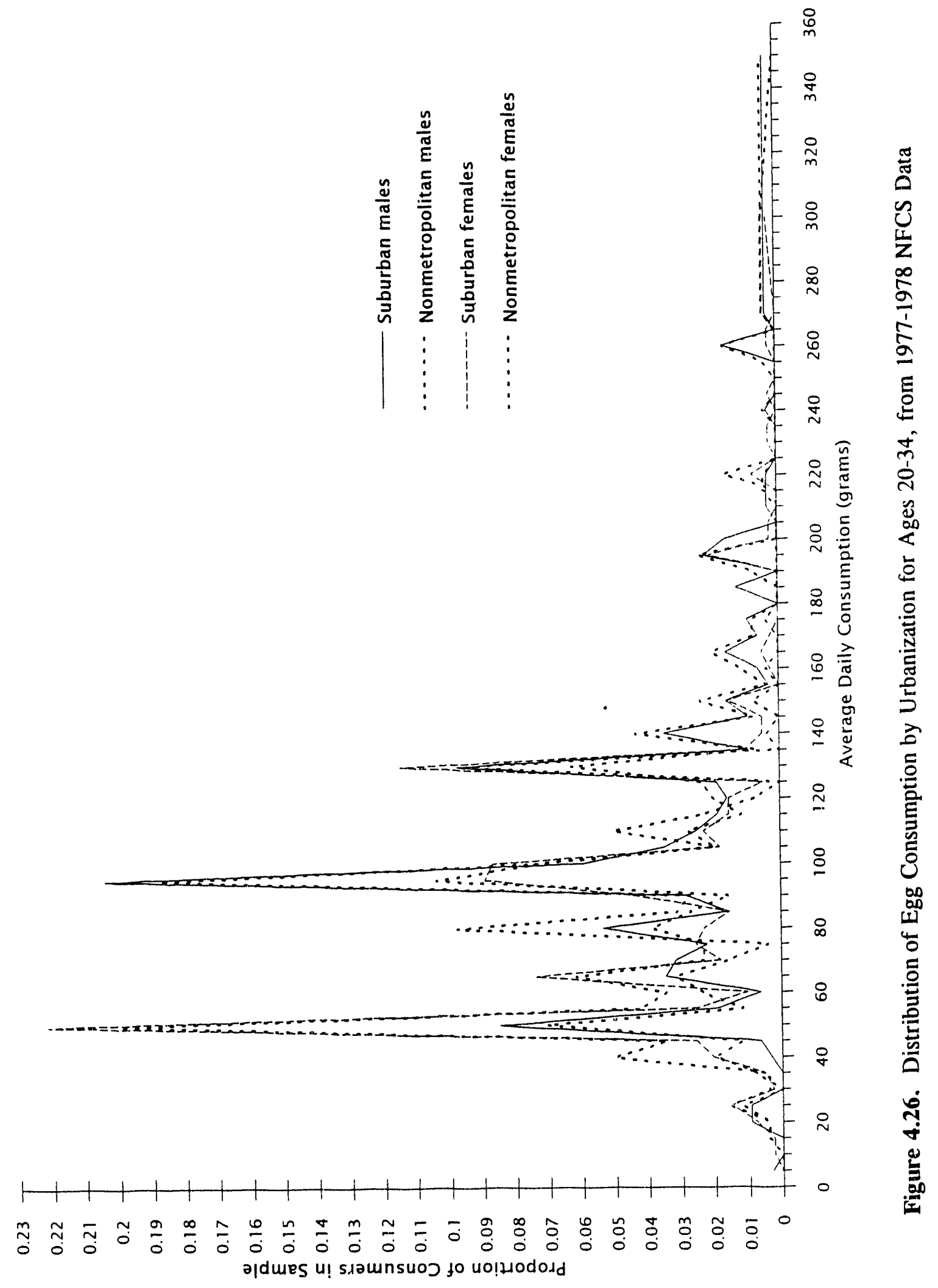




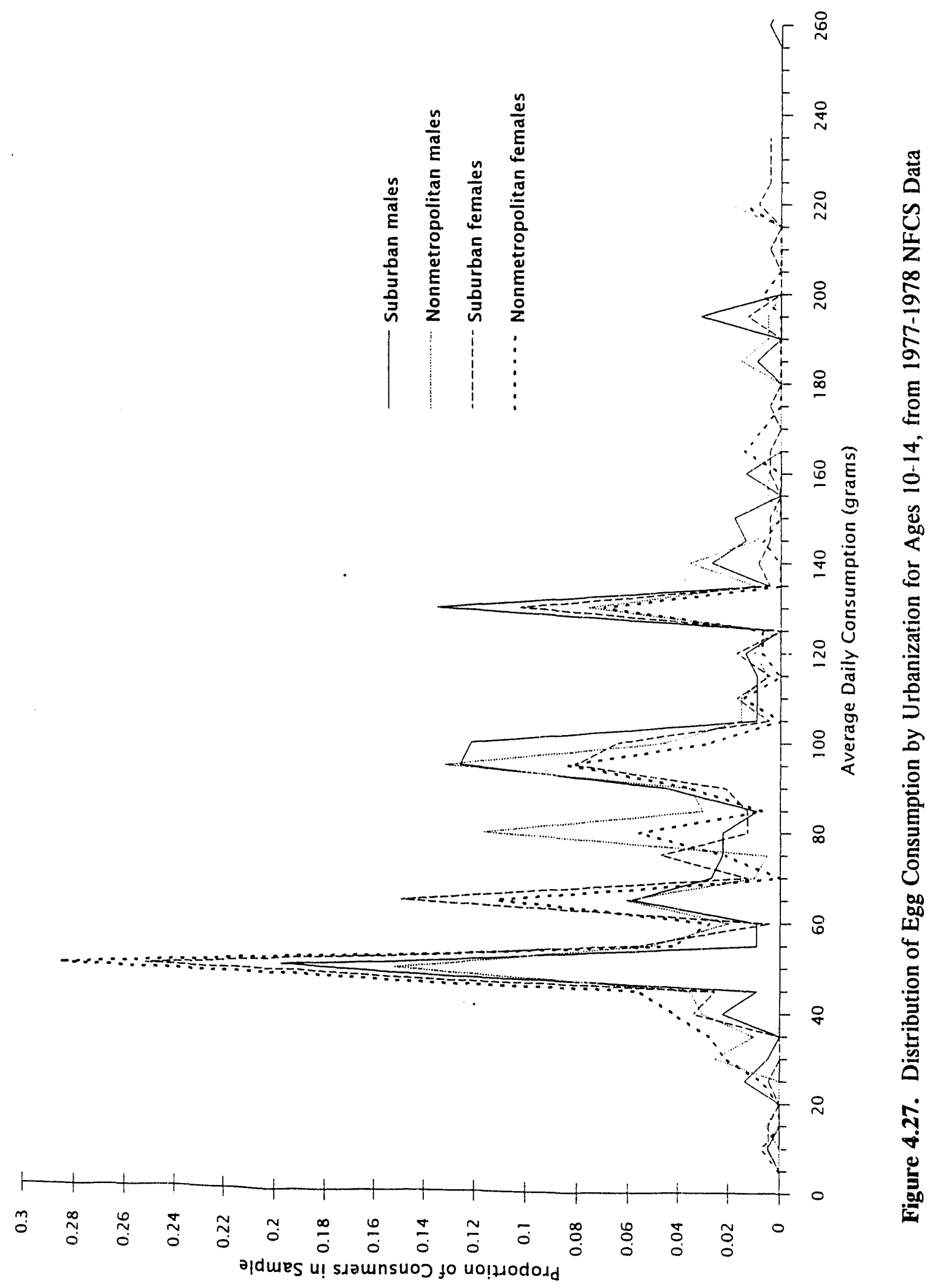




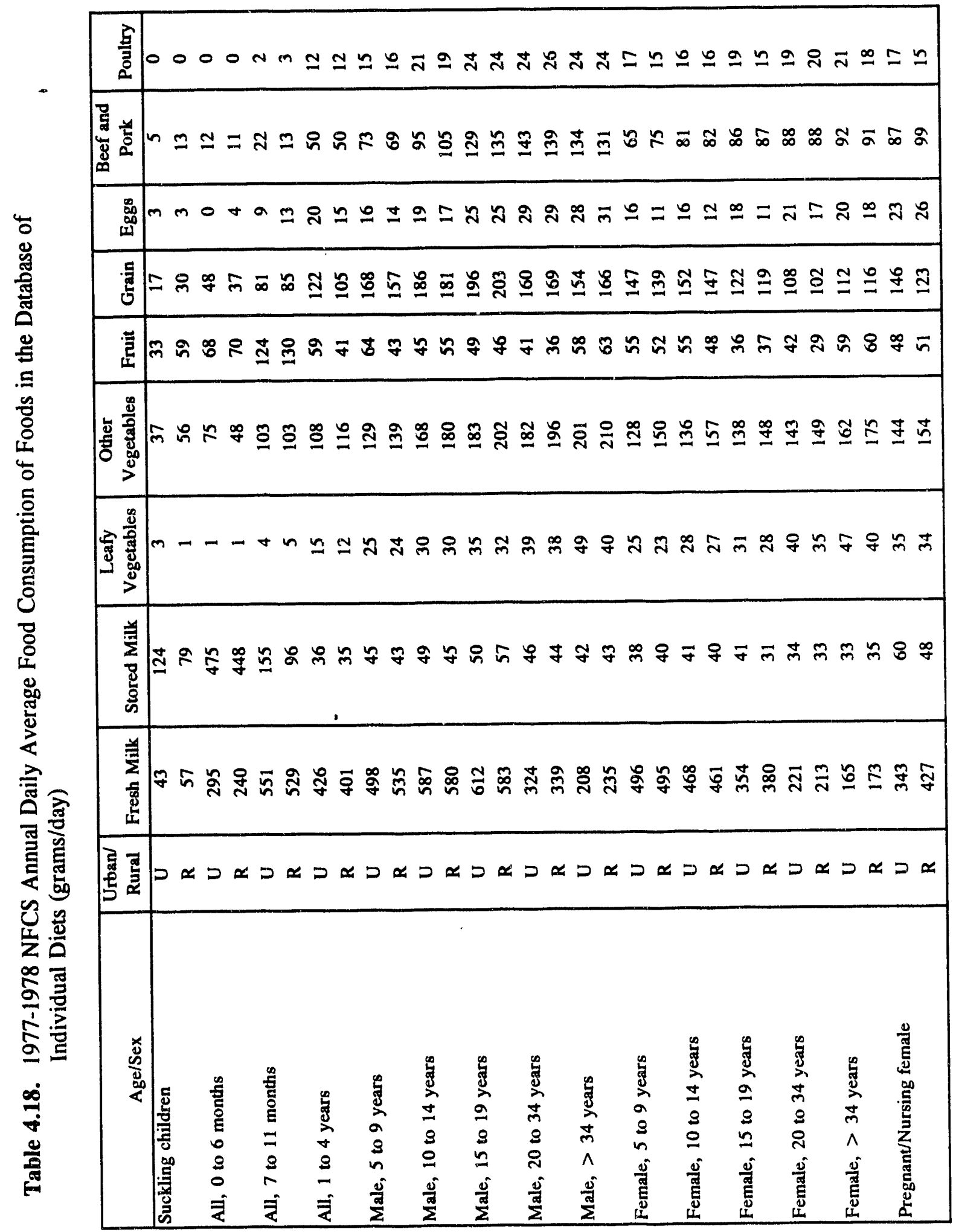




\subsection{References}

Anderson, D. M. 1992. Methodology for Reconstruction of Historical Food Consumption Estimates. PNL-8123 HEDR, Pacific Northwest Laboratory, Richland, Washington.

Bustad, L. K., and J. L. Terry. 1956. Basic Anatomical, Dietary, and Physiological Data for Radiological Calculations. HW-41638, General Electric Company, Hanford Atomic Products Operation, Richland, Washington.

Callaway, J. M. 1992. Estimation of Food Consumption. PNL-7260 HEDR, Pacific Northwest Laboratory, Richland, Washington.

Clark, F. and C. B. LeBovit. 1955. Food Consumption of Farm Families, Meeker and Wright Counties, Minnesota, 1950. USDA Agriculture Information Bulletin 127, Washington, D.C.

Durbin, P. W., J. Lynch, and S. Murray. 1970. "Average Milk and Mineral Intakes (Cailcium, Phosphorus, Sodium and Potassium) of Infants in the United States from 1954 to 1968; Implications for Estimating Annual Intakes of Radionuclides." Health Physics. 19 (2): 187-222. Pergamon Press Ltd., New York, New York.

Endres, G. W. R., J. K. Soldat, D. B. Shipler, N. M. Robinson, F. N. Eichner, and J. F. Honstead. 1972. Dietary and Body Burden Data and Dose Estimates for Local School Children and Teenagers. Research report for the Environmental Protection Agency, Battelle Pacific Northwest Laboratories, Richland, Washington.

Eslinger, P. W., T. B. Miley, S. J. Ouderkirk, and W. E. Nichols. 1992. Preliminary Design Studies for the Descartes and Cider Codes. BN-SA-368 HEDR, Battelle Pacific Northwest Laboratories, Richland, Washington.

Honstead, J. F. 1966. Dietary Sources of Radioactivity for Richland Residents. BNWL-CC-926, Battelle Northwest, Richland, Washington.

Honstead, J. F. 1967. Radionuclide Burden-Diet Relationships Near a Nuclear Facility. BNWL-SA1251, Battelle Northwest, Richland, Washington.

Ikenberry, T. A., R. A. Burnett, B. A. Napier, N. A. Reitz, and D. B. Shipler. 1992. Integrated Codes for Estimating Environmental Accumulation and Individual Dose from Past Hanford Atmospheric Releases. PNL-7993 HEDR, Pacific Northwest Laboratory, Richland, Washington.

Manchester, A. C., K. R. Farrell. 1981. Measurement and Forecasting of Food Consumption by USDA. In: Assessing Changing Food Consumption Patterns, Committee on Food Consumption Patterns, pp. 51-71. National Academy Press, Washington, D.C. 
Marsh, T. L., D. M. Anderson, W. T. Farris, T. A. Ikenberry, B. A. Napier, and G. L. Wilfert. 1992. Commercial Production and Distribution of Fresh Fruits and Vegetables: A Scoping Study on the Importance of Produce Pathways to Dose. PNWD-2022 HEDR, Battelle Pacific Northwest Laboratories, Richland, Washington.

Napier, B. A. 1992a. Determination of Radionuclides and Pathways Contributing to Cumulative Dose. BN-SA-3673 HEDR, Battelle Pacific Northwest Division, Richland, Washington.

Napier, B.A. 1992b. Determination of Radionuclides and Pathways Contributing to Dose in 1945. BN-SA-3674 HEDR, Battelle Pacific Northwest Division, Richland, Washington.

Napier, B. A., J. C. Simpson, T. A. Ikenberry, R. A. Burnett, T. B. Miley. 1992. Final Design Specification for the Environmental Pathways and Dose Model. PNL-7844 HEDR, Pacific Northwest Laboratory, Richland, Washington.

Nelson, C. B., and Y. Yang. 1984. An Estimation of the Daily Food Intake Based on Data From the 1977-1978 USDA Nationwide Food Consumption Survey. EPA 520/1-84-015, U.S. Environmental Protection Agency, Office of Radiation Programs, Washington, D.C.

Pacific Northwest Laboratory (PNL). 1991. Air Pathway Report: Phase I of the HEDR Project. PNL-7412 HEDR Rev. 1, Richland, Washington.

SAS 1989, SAS/STAT User's Guide, Version 6, Fourth Edition, Volumes 1 and 2, SAS Institute Inc., Cary, North Carolina.

Shipler, D. B. 1992. Integrated Task Plans for the Hanford Environmental Dose Reconstruction Project, FY 1992 Through May 1994. PNWD-2020 HEDR, Pacific Northwest Laboratory, Richland, Washington.

Shipler, D. B., G. W. R. Endres, N. M. Robinson, T. H. Essig, and J. F. Honstead. 1972. Results of $a$ Whole Body Counting Study of Families Living on Irrigated Farms Downstream of the Hanford Project. Research report for the Environmental Protection Agency, Battelle Pacific Northwest Laboratories, Richland, Washington.

Shleien, B. 1992. Scoping Document for Determination of Temporal and Geographic Domains for the HEDR Project. Washington State Department of Ecology, Olympia, Washington.

Snyder, S. F., W. T. Farris, B. A. Napier, T. A. Ikenberry, and R. O. Gilbert. 1992. Parameters Used in the Environmental Pathways (DESCARTES) and Radiological (CIDER) Modules of the Hanford Environmental Dose Reconstruction Integrated Code (HEDRIC) Dose for the Air Pathway. PNWD-2023 HEDR, Battelle Pacific Northwest Division, Richland, Washington.

Soldat, J. K., and J. F. Honstead 1968. Dietary Levels for Tri-City Elementary School Children. BNWL-CC-1565, Battelle Pacific Northwest Laboratories, Richland, Washington. 
U.S. Department of Agriculture (USDA). 1941. Family Food Consumption and Dietary Levels: Five Regions. Miscellaneous Publication 405, Bureau of Home Economics, Washington, D.C.

U.S. Department of Agriculture (USDA). 1944. Family Food Consumption in the United States: Spring 1942. Miscellaneous Publication 550, Bureau of Human Nutrition and Home Economics, Agricultural Research Administration, Washington, D.C.

U.S. Department of Agriculture (USDA). 1955a. Food Consumption of Households in the West. Household Food Consumption Survey 1955, Report \#5, Washington, D.C.

U.S. Department of Agriculture (USDA). 1955b. Food Production for Home Use in the United States by Region. Household Food Consumption Survey 1955, Report \#12, Washington, D.C.

U.S. Department of Agriculture (USDA). 1965. U.S. Food Consumption: Sources of Data and Trends 1909 - 1963. Statistical Bulletin 364, Economic Research Service, Washington, D.C.

U.S. Department of Agriculture (USDA). 1972. Individual Intake Data on Magnetic Tape for Spring 1965. Household Food Consumption Survey 1965-1966, Health and Nutrition Information Service, Washington, D.C.

U.S. Department of Agriculture (USDA). 1979. Coding Manual to Handle Data from Nationwide Survey of Individuals. USDA/DF-80/004a, Consumer Food Economics Institute, Hyattsville, Maryland.

U.S. Department of Agriculture (USDA). 1981. Food Consumption, Prices, and Expenditures. Statistical Bulletin 656, Economic Research Service, Washington, D.C.

U.S. Department of Agriculture (USDA). 1983. Food Intakes: Individuals in 48 States, Year $1977-$ 78. Nationwide Food Consumption Survey 1977-78, Report I-1 and associated raw survey data on magnetic tape, Human Nutrition Information Service, Hyattsville, Maryland. 
Appendix A

\section{Final Food Groupings}




\section{Appendix A}

\section{Final Food Groupings}

This appendix contains the 65 potential foods that were analyzed to obtain consumption estimates. A brief description of the specific foods in each group is provided. These groups were formed by aggregating 3,735 coded foods from the 1977-1978 NFCS that are described in USDA (1979). The description of the resulting 65 food groups analyzed appears in Table A.1. Initially 75 groups were identified for analysis, but lack of a sufficient number of survey observations caused the reclassification of 15 groups into 5 groups, reducing the number of groups to 65 . This reclassification is shown in Table A.2. Table A.3 depicts the aggregation to the level used in the database of individual diets for input to the dose-calculation model. Most of the food group titles for the dose-calculation model are self explanatory. However, it should be noted that vegetables such as beans, cauliflower, and peas are classified as "leafy vegetables" because they, like most leafy vegetables, have large, exposed, edible surfaces.

Table A.1. Sixty-Five Food Groups Analyzed to Obtain Consumption Estimates

\begin{tabular}{|c|c|}
\hline \multicolumn{2}{|c|}{ Dairy Products } \\
\hline 1 & $\begin{array}{c}\text { Fresh milk } \\
\text { Fluid milk, fluid milk used as a major ingredient, puddings and } \\
\text { other fresh milk desserts, milk-reconstituted dry milk products }\end{array}$ \\
2 & $\begin{array}{l}\text { Fresh cream } \\
\text { Fluid cream, whipping cream, butter, buttermilk, fluid cream } \\
\text { used as a major ingredient (salad dresings, pies) }\end{array}$ \\
4 & $\begin{array}{l}\text { Cottage cheese } \\
\text { Other cheese } \\
\text { All noncottage types of cheese }\end{array}$ \\
6 & $\begin{array}{l}\text { Ice cream and other frozen desserts } \\
\text { All ice cream and yogurt } \\
7\end{array}$ \\
$\begin{array}{l}\text { Baby formula and canned milk products } \\
\text { Fluid baby formula (nonmilk), evaporated milk, condensed milk } \\
\text { Dried milk products } \\
\text { All dry milk products and water-reconstituted dry milk products }\end{array}$ \\
Goat milk
\end{tabular}

A. 1 
Table A.1. (contd)

Fresh Vegetables Including Fresh-Cooked

\begin{tabular}{|c|c|}
\hline 9 & Green peas \\
\hline 10 & Corn \\
\hline 11 & Asparagus \\
\hline 12 & Tomatoes \\
\hline 13 & Snap beans \\
\hline 14 & Cabbage \\
\hline 15 & Lettuce \\
\hline 16 & Spinach \\
\hline 17 & Cauliflower \\
\hline 18 & Celery \\
\hline 19 & Broccoli \\
\hline 20 & Carrots \\
\hline 21 & Onions \\
\hline 22 & Beets \\
\hline 23 & Turnips, rutabagas \\
\hline 24 & Squash, pumpkin \\
\hline 25 & Cucumbers \\
\hline 26 & Green peppers \\
\hline 27 & Lima beans \\
\hline 28 & Leafy vegetables not classified elsewhere \\
\hline & $\begin{array}{l}\text { Beet greens, chard, kale, collards, cress, endive, dandelion } \\
\text { greens, escarole, etc. }\end{array}$ \\
\hline 29 & Other fresh vegetables not classified elsewhere \\
\hline & Chives, eggplant, mushrooms, radishes, etc. \\
\hline \multicolumn{2}{|c|}{ Canned and Frozen Vegetables } \\
\hline 30 & Tomatoes \\
\hline 31 & Stored vegetables \\
\hline 32 & Vegetable mixes, soups \\
\hline & Mixed vegetables, vegetable soups, vegetable dishes, etc. \\
\hline \multicolumn{2}{|c|}{ Other Vegetables } \\
\hline 33 & Fresh white potatoes \\
\hline 34 & Fresh sweet potatoes \\
\hline 35 & Processed potatoes \\
\hline & Canned and frozen potatoes \\
\hline 36 & Vegetable juices \\
\hline
\end{tabular}

\section{A. 2}


Table A.1. (contd)

\section{Fruits}

Pears

Strawberries

40 Other berries

Raspberries, blackberries, blueberries, loganberries, etc.

Sweet cherries

42 Melons

Watermelons, cantaloupe, etc.

43 Grapes

44 Apricots

45 Peaches

46 Plums (Italian prunes)

Other noncitrus (excluding banana and pineapple)

All other locally grown fruits, fruit salads or mixes, etc.

48 Noncitrus fruit juices (excluding banana and pineapple)

Fresh Meat and Eggs

49 Beef

50 Pork

51 Chicken

52 Other poultry

Turkey, cornish hen, etc.

54

Venison, rabbit, duck, goose, quail, etc.

Cured pork

Smoked ham, bacon, sausage, etc.

Lunchmeat

Prepared sandwich meats like bologna, salami, etc. 
Table A.1. (contd)

\begin{tabular}{|r|r|}
\hline Fresh Seafood \\
\hline 58 & $\begin{array}{c}\text { Local fish } \\
\text { Salmon, sturgeon, trout, bass, catfish, crappie, etc. } \\
\text { Other fish } \\
\text { Shark, pike, cod, halibut, eel, frog legs, squid, etc. } \\
60\end{array}$ \\
\hline Shell fish \\
\hline 61 & $\begin{array}{c}\text { Bread and rolls } \\
\text { Other baked products } \\
\text { Pie shells, pasta, cake desserts, etc. }\end{array}$ \\
\hline 63 & $\begin{array}{c}\text { Mixtures } \\
\text { Mixed dishes such as frozen plate meals (TV dinners), } \\
\text { casseroles, meat and vegetable mixtures, soups, etc. }\end{array}$ \\
\hline 64 & $\begin{array}{c}\text { All other foods } \\
\text { Fats, sugars, sweeteners, coffee, prepared drinks, soft drinks, } \\
\text { alcoholic beverages, exotic fruits, breakfast cereals, candy, etc. }\end{array}$ \\
\hline 65 & Human milk \\
\hline
\end{tabular}

A. 4 
Table A.2. Food Types Regrouped Based on Insufficient Number of Observations to Permit Meaningful Statistical Analysis

\begin{tabular}{|c|l|}
\hline New Food Type & \multicolumn{1}{|c|}{ Previous Food Types } \\
\hline Fresh onions & $\begin{array}{l}\text { Fresh green onions } \\
\text { Fresh other onions }\end{array}$ \\
$\begin{array}{r}\text { Stored leafy } \\
\text { vegetables }\end{array}$ & $\begin{array}{l}\text { Spinach, canned/frozen } \\
\text { Leafy vegetables, } \\
\text { canned/frozen } \\
\text { Peas, canned/frozen } \\
\text { Snap beans, canned/frozen } \\
\text { Snap beans, canned/frozen }\end{array}$ \\
Stored other \\
vegetables \\
Corn, canned/frozen \\
Other vegetables \\
Dried vegetables \\
Other fish & $\begin{array}{l}\text { Game meat } \\
\text { Game poultry }\end{array}$ \\
Bread and rolls & $\begin{array}{l}\text { Other fish } \\
\text { Other seafood }\end{array}$ \\
Flour and prepared mixes \\
Bread and rolls
\end{tabular}


Table A.3. Translation of Foods Reported in this Study to Their Equivalent Database Aggregation for Use in Dose Calculation

\begin{tabular}{|l|l|}
\hline \multicolumn{1}{|c|}{ Group of 65} & Database Aggregate \\
\hline Fresh milk and as ingredient & Fresh milk \\
Cream and as ingredient & Fresh milk \\
Cottage cheese & Fresh milk \\
Other cheese & Stored milk \\
Ice cream \& other frozen deserts & Stored milk \\
Baby formula and canned milk products & Stored milk \\
Dried milk products & Stored milk \\
Goat milk & Fresh milk \\
Fresh green peas & Leafy vegetables \\
Fresh corn & Other vegetables \\
Fresh asparagus & Other vegetables \\
Fresh tomatoes & Other vegetables \\
Fresh snap beans & Leafy vegetables \\
Fresh cabbage & Leafy vegetables \\
Fresh lettuce & Leafy vegetables \\
Fresh spinach & Leafy vegetables \\
Fresh cauliflower & Leafy vegetables \\
Fresh celery & Leafy vegetables \\
Fresh broccoli & Leafy vegetables \\
Fresh carrots & Other vegetables \\
Fresh onions & Other vegetables \\
Fresh beets & Other vegetables \\
Fresh turnips, rutabagas & Other vegetables \\
Squash, pumpkin & Other vegetables \\
Frssh cucumbers & Other vegetables \\
Fresh green peppers & Other vegetables \\
Fresh lima beans & Other vegetables \\
Other fresh leafy vegetables & Leafy vegetables \\
Other fresh vegetables & Other vegetables \\
Tomatoes, canned/frozen & Other vegetables \\
Stored vegetables & Leafy Vegetables/ \\
Vegetable mixes, soups & other vegetables \\
Fresh white potatoes & Other vegetables \\
& Other vegetables \\
\hline
\end{tabular}


Table A.3. (contd)

\begin{tabular}{|l|l|}
\hline \multicolumn{1}{|c|}{ Group of 65 } & Database Aggregate \\
\hline Processed potatoes & Other vegetables \\
Vegetable juices & Other vegetables \\
Apples & Other vegetables \\
Pears & Fruit \\
Strawberries & Fruit \\
Other berries & Fruit \\
Sweet cherries & Fruit \\
Melons & Fruit \\
Grapes & Fruit \\
Apricots & Fruit \\
Peaches & Fruit \\
Plums/prunes & Fruit \\
Other noncitrus fruits & Fruit \\
Noncitrus fruit juices & Fruit \\
Beef & Beef/Pork \\
Pork & Beef/Pork \\
Chicken & Poultry \\
Other poultry & Poultry \\
Game; deer, elk, rabbit, goose, duck & Becf/Pork \\
Cured pork & Not included \\
Lunch meat & Not included \\
Other fresh meat & Beef/Pork \\
Eggs & Eggs \\
Local fish & Not included \\
Other fish & Not included \\
Shellfish & Not included \\
Bre:d and rolls & Grain \\
Other bakery products & Grain \\
Food mixtures & Not included \\
Other food & Not included \\
Human milk & Not included \\
\hline & \\
\hline
\end{tabular}


Appendix B

Backcasting 1977-1978 Consumption Estimates to 1945-1957 


\section{Appendix B}

\section{Backcasting 1977-1978 Consumption Estimates to 1945-1957}

\section{Introduction}

Under ideal circumstances, survey data for individual food intake reported for population subgroups would have been collected in 1945-1957 for the counties of interest. Because average daily ingestion rates for specific food types that distinguish among socioeconomic characteristics, seasonal consumption differences, and urban/rural attributes of populations were not available for this period, backcasting ratios were constructed earlier (Callaway 1992) to convert (backcast) average daily ingestion rates from 1977 to 1945 . Average daily ingestion rate estimates (based on age, sex, season, and urban/rural status) constructed from the raw data of the 1977-1978 NFCS (USDA 1983) were used as the baseline consumption estimates. The backcasting ratios for individual food types were constructed from a consistent annual time series of per capita gross disappearance estimates maintained by the USDA for most of this century (USDA 1965 and 1981). The annual gross disappearance estimates for a given food type are the consumption of foud per capita in weights at the retail level. The use of backcasting ratios in the calculation of food consumption estimates assumes that the percentage of change in the average daily consumption of food from 1945-1957 in the HEDR region is proportional to the percentage of change in the U.S. average per capita daily gross disappearance estimates.

The objective of this analysis is to consider the application of the backcasting ratio as a way to project food ingestion estimates into the past. As indicated by Anderson (1992, pp.3-4) such an analysis should include a discussion of the ratio backcasting methodology to estimate historical levels of food consumption, validate the calculated estimates using other secondary sources, and address the question of the uncertainty that the backcasting ratios introduce into food consumption estimates.

Of particular interest are the average daily ingestion rates for the years 1945-1957, the selected reference years in the study period of this report. Because the estimated average ingestion rates are backcasted from the 1977-1978 NFCS data to 1945-1957, the reliability of these estimates needs to be examined. The estimates of fresh milk consumption are of greatest interest because milk has been found to be potentially the most significant contributor to dose from iodine-131 (Napier 1992b). This appendix will focus on the ability of the backcasting ratios to reliably estimate food consumption in the 1945-1957 period. 


\section{Data on Retail Disappearance of Food}

Annual per capita civilian consumption data are estimated in USDA (1965 and 1981). These annual estimates are compiled from balance sheet tables that attempt to equate weights of farm-level production of food (supply) with the weights of food that "disappear" into the marketing and distribution system (utilization). Food supply includes stocks at the beginning of the year, annual production, and imports. Food utilization includes exports and the various domestic end-uses such as non-food uses, military consumption, and civilian consumption. Civilian food consumption is the residual supply after subtracting all other known uses and provides the basis for the annual average per capita consumption estimate for a given food. The per capita calculation is based on the estimated July 1 population of the United States. The civilian consumption of a given food is derived by calculating a retail equivalent of the food's primary weight or weight at which the food enters the distribution system from farm production. The total retail weight of the civilian supply of the food is divided by the U.S. population on July 1 to obtain national average consumption per capita of the given food in a given year.

This means all statistical and measurement error that accumulates through the calculations from farm production to per capita consumption is passed to these estimates (USDA 1965). Because the backcasting ratios are calculated directly from the national per capita consumption estimates, they also receive the sum of all error incurred. However, the same level of error is incurred each year, which gives the estimates from 1 year relative to another usefulness in determining national trends for specific foods. This relative comparison of food-specific per capita consumption in any 2 years, $x$ and $y$, yields a factor that, when multiplied by consumption in year $x$, yields consumption in year $y$. This factor is the backcasting ratio and is calculated for any food as follows:

$$
R_{i_{x}}=\frac{D_{i_{x}}}{D_{i_{y}}}
$$

where

$$
\begin{aligned}
& R_{i_{x}}=\text { backcasting ratio for food } \mathrm{i} \text { for converting consumption in year } \mathrm{y} \text { to year } \mathrm{x} \\
& D_{i_{x}}=\text { national per capita consumption (retail disappearance) of food } \mathrm{i} \text { in year } \mathrm{x} \\
& D_{i_{y}}=\text { national per capita consumption (retail disappearance) of food } \mathrm{i} \text { in year } \mathrm{y} \text { (the } \\
& \text { reference year). }
\end{aligned}
$$

\section{Sources of Introduced Error in Backcasting Ratios}

Uncertainty exists in the backcasting ratios because it is inherent in the underlying per capita consumption values used to construct the ratios. No quantification of statistical or measurement error is available for the national per capita consumption data. However, it is possible to characterize what type of error occurs at the calculation steps leading from farm production to civilian consumption. 
Data are required for the years of interest (1945-1957) and the reference year for which NFCS data were collected (1977). Measurement errors occur when any source of consumption is omitted from calculations or when consumption reported in official documents or receipts differs from what actually took place. Some military consumption data include amounts that were consumed by civilians partaking of military supplies, but the incidence or error value is not given. Additional measurement error occurs when home production of foods, the food use of wild fish and game, and manufacture of secondary and retail products are not adequately measured in food consumption terms (Manchester and Farrell 1981).

Surveys have also been used extensively to collect consumption data at various stages of food utilization. Statistical error is introduced through sampling error any time surveys are employed. Statistical error also enters the utilization calculations that carry rounded values through different calculation stages. Additional error occurs when census data are used to estimate annual averages for the inter-census period. Because civilian consumption is the net of supply after all other end-uses have been deducted, total food available for civilian consumption is dependent on the error passed through all the calculations leading up to that point. Deriving per capita estimates using the estimated July 1 population incurs additional error from the error in the population census and subsequent estimations of non-census-year and mid-year population.

There is no quantitative measurement available of the cumulative uncertainty inherent in the backcasting ratios. It should be noted that, given the numerous sources of error attributable to any national average per capita consumption estimate, the error is consistent. The same statistical and measurement errors are carried through the calculations of civilian consumption year after year.

Evaluating this food-specific time series of per capita consumption in relative terms is useful for estimating consumption trends. Table B.1 provides the backcasting ratios developed for the analysis in this report and the source of the data used to estimate them. Figures B.1-B.4 present backcasted estimates of consumption for the four dose-relevant foods analyzed in the report. These estimates are compared with estimates of per capita consumption from local and other national data. They compare backcasted NFCS estimates with estimates from the 1965 HFCS data (USDA 1972) for fresh milk, eggs, lettuce, and spinach for specific age/sex classifications. The backcasting method comes closest in estimating the actual HFCS values for the fresh milk group and is less reliable for estimating fresh spinach consumption. 


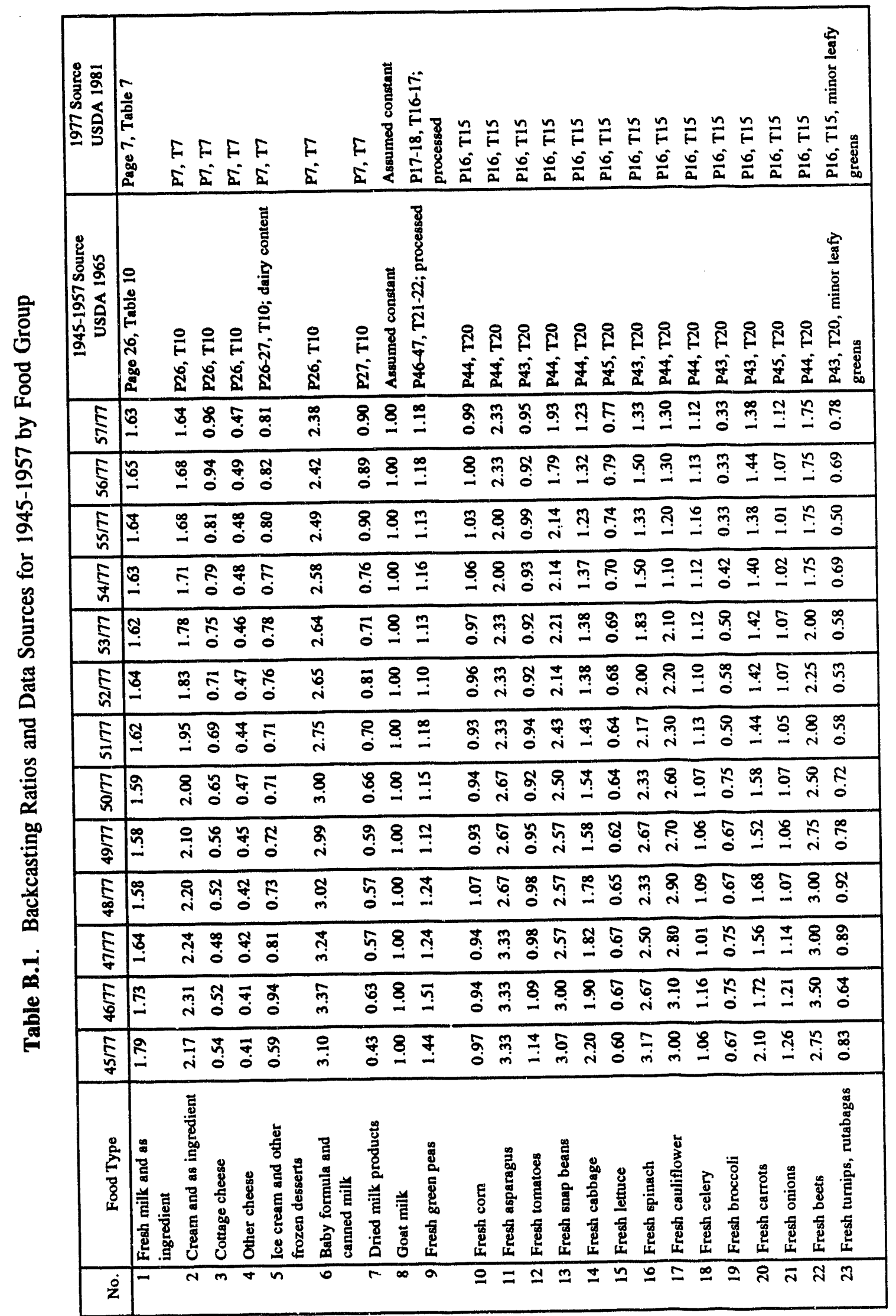




\begin{tabular}{|c|c|c|c|c|c|c|c|}
\hline 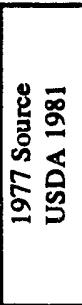 & 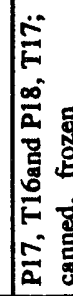 & 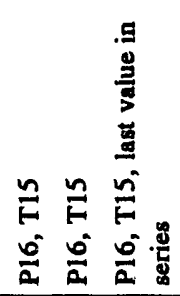 & & $\begin{array}{l}\frac{n}{a} \\
\stackrel{s}{a}\end{array}$ & 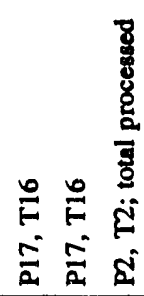 & 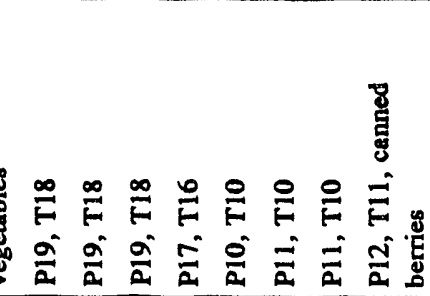 & 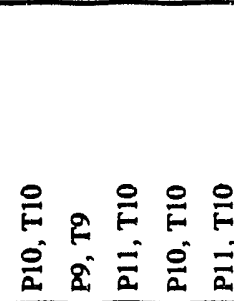 \\
\hline 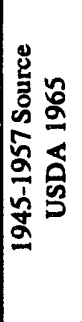 & 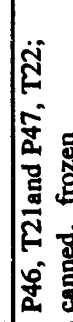 & 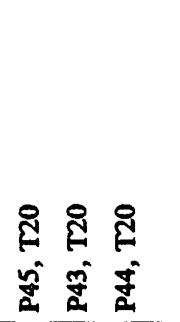 & 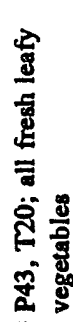 & 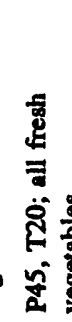 & 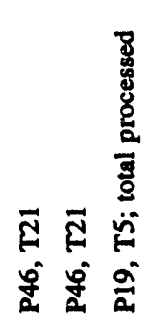 & 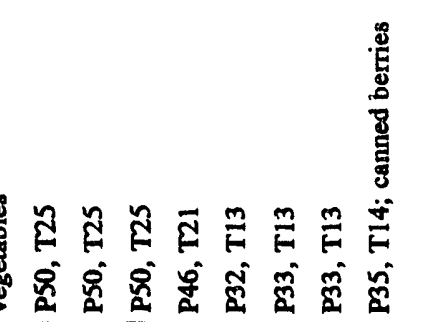 & 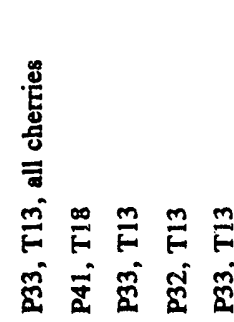 \\
\hline 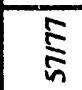 & דa & $\stackrel{0}{0} 8$ & : & $\stackrel{t}{g}$ & : & వ్ & 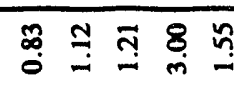 \\
\hline 趈 & 8 & : & 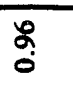 & ִֻ & $\begin{array}{lll}m & 0 & 0 \\
0 & 0 & 0 \\
0 & 0 & 0\end{array}$ & నิ & 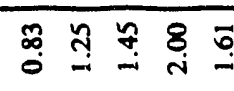 \\
\hline 点 & 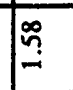 & 竞: & $\stackrel{\substack{a \\
0}}{0}$ & 8 & $\begin{array}{lll}0 & 0 \\
& 0 & 0 \\
0 & 0 \\
0\end{array}$ & 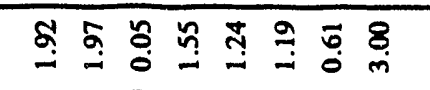 & 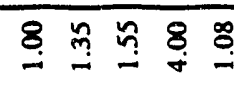 \\
\hline 甭 & 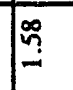 & 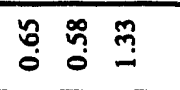 & $\stackrel{\circ}{\circ}$ & 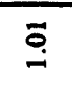 & 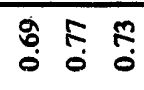 & 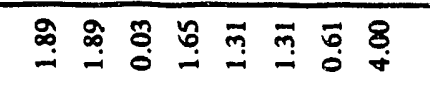 & $8 \bar{m} \stackrel{0}{9} \stackrel{9}{9}$ \\
\hline F & 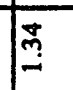 & 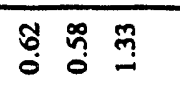 & $\stackrel{\text { ă }}{o}$ & ș & c: & 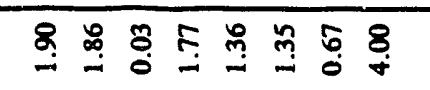 & 8 용 \\
\hline$\underset{\text { F్ }}{\mathbb{N}}$ & $\tilde{n}$ & 芯点 & $\stackrel{2}{\circ}$ & $\stackrel{t}{\sigma}$ & $\begin{array}{lll}0 & 0 \\
0 & 0 & 0 \\
0 & 0 & 0\end{array}$ & 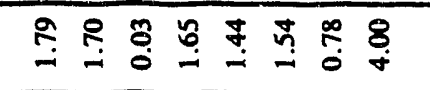 & $\fallingdotseq \stackrel{9}{9} 8 \stackrel{\circ}{9}$ \\
\hline$\frac{5}{n}$ & ?ָ- & ڤิ & ă & ț & 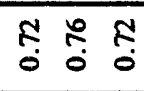 & 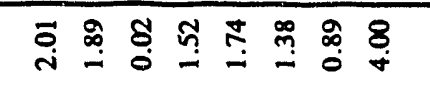 & $8 \fallingdotseq \stackrel{000}{9}$ \\
\hline F & 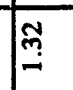 & $\overline{5}$ & $\stackrel{9}{9}$ & 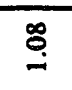 & $\begin{array}{lll}R & ? \\
0 & ? \\
0 & 0 \\
0\end{array}$ & 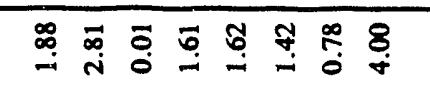 & $\exists \equiv \underset{9}{8} \stackrel{n}{m}$ \\
\hline$\frac{F}{9}$ & 8 & ڤิ̂ & 8 & 品 & $\begin{array}{lll}t & 0 & n \\
0 & 0 & 0 \\
0\end{array}$ & ๖ำ & 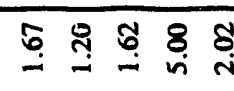 \\
\hline$\underset{\substack{F \\
\text { F }}}{F}$ & $\stackrel{p}{?}$ & 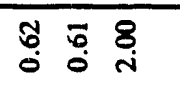 & $\stackrel{2}{\longrightarrow}$ & $\dddot{n}$ & $\begin{array}{lll}8 & 8 & 0 \\
0 & 0 & 0 \\
0\end{array}$ & 市 & $\Xi$ I $98 \%$ \\
\hline 点 & 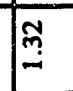 & ڤ్̂े & $\Xi$ & 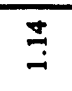 & $\begin{array}{lll}0 & 0 & 0 \\
0 & 1 & 5 \\
0\end{array}$ & స్ํ & कृ \\
\hline $\bar{E}$ & דִיְ: & 范 & $\stackrel{m}{=}$ & $\bar{ָ}$ & $\begin{array}{lll}F & 0 & 0 \\
0 & 0 & 0 \\
0 & 0\end{array}$ & 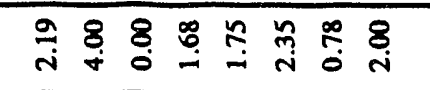 & $\stackrel{0}{6} \stackrel{9}{9} \stackrel{8}{9}$ \\
\hline$\frac{F}{9}$ & : & in & $\stackrel{\widetilde{m}}{2}$ & $\stackrel{\dddot{n}}{\dddot{n}}$ & \begin{tabular}{lll}
$\overline{0}$ & $n$ & \multirow{2}{n}{} \\
0 & 0 & 0 \\
0 & 0
\end{tabular} & 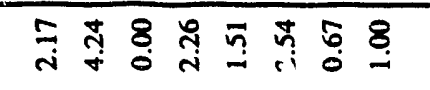 & 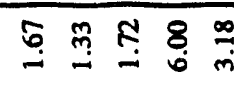 \\
\hline 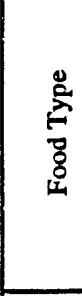 & 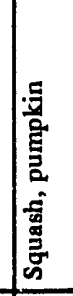 & 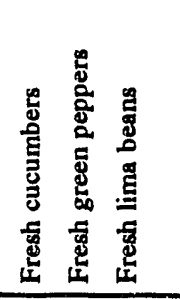 & 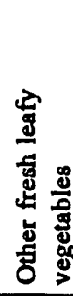 & 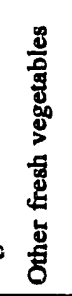 & 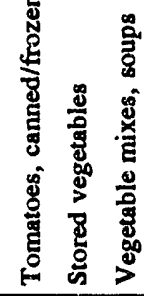 & 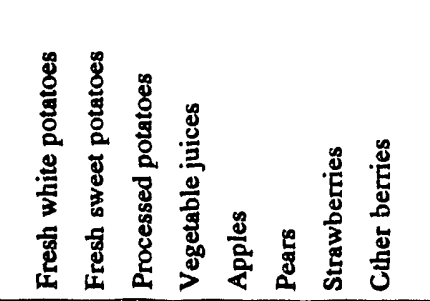 & 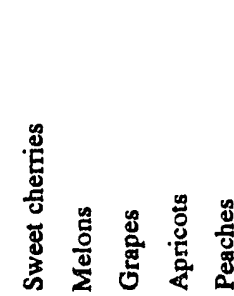 \\
\hline$\dot{s}$ & $\frac{4}{4}$ & $\frac{4}{4} \sqrt{4}$ & iి & สิ & $\vec{\rho} \bar{m} \tilde{m}$ & 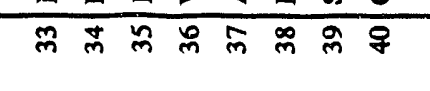 & 79878 \\
\hline
\end{tabular}




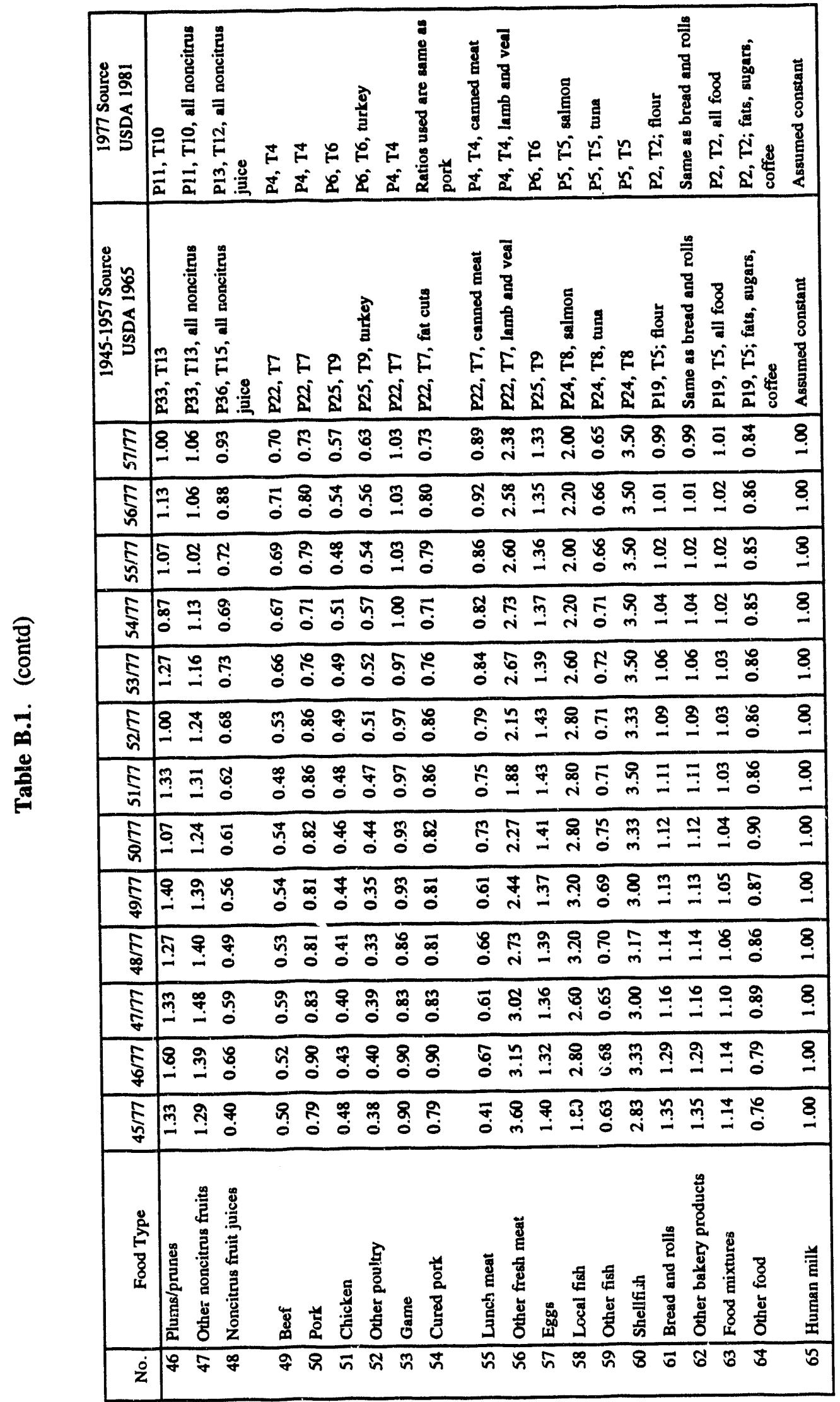

B. 6 


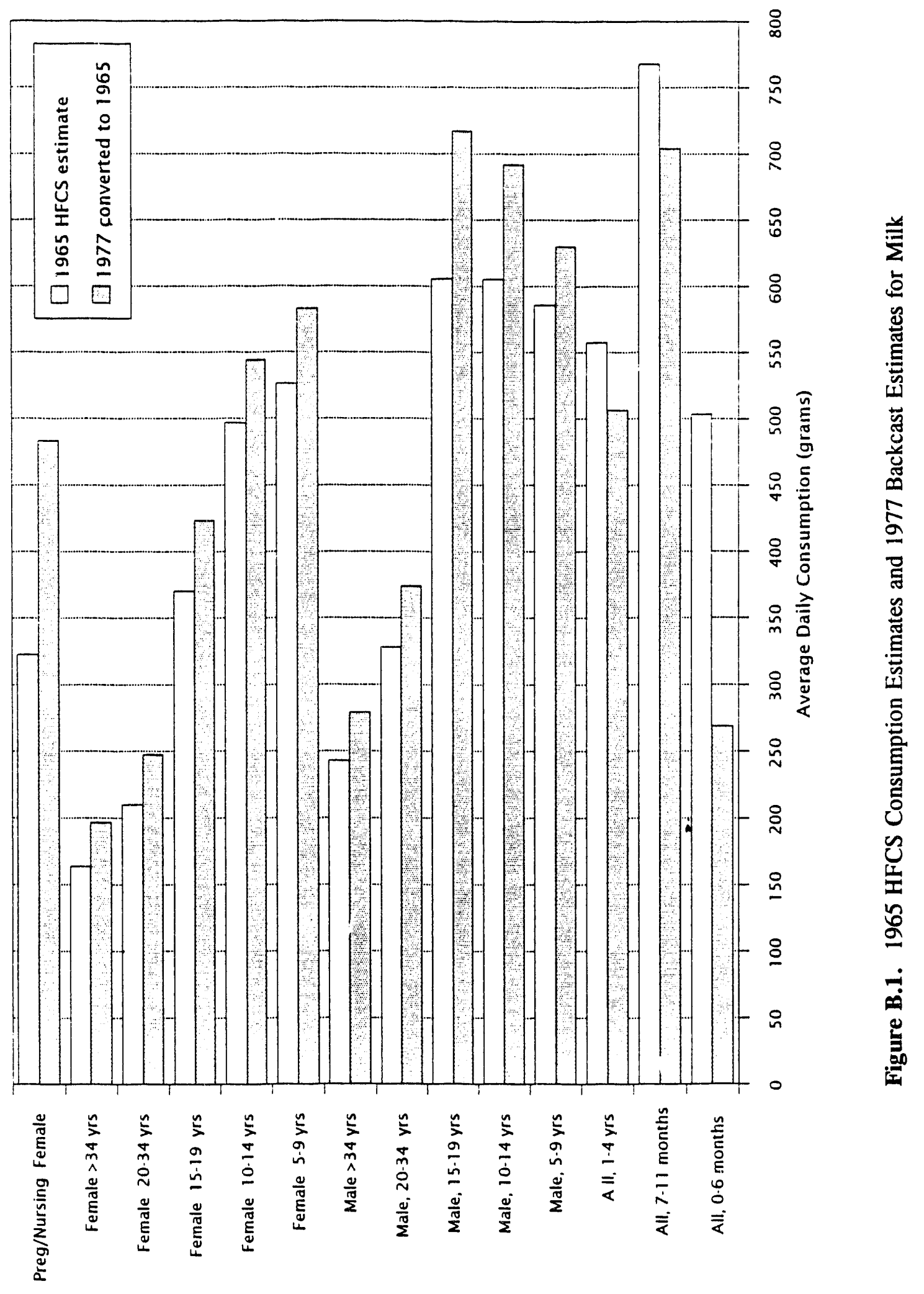

B. 7 


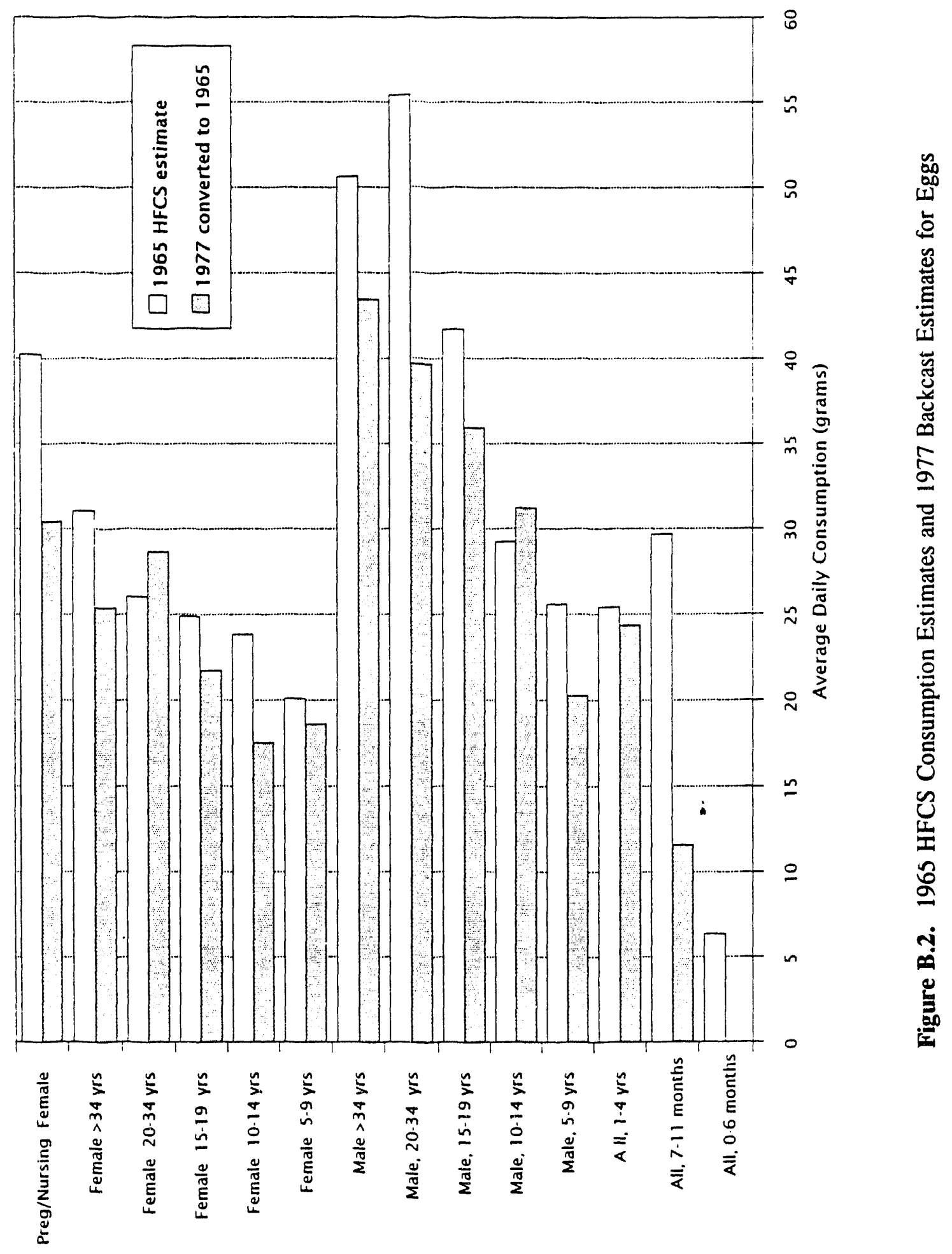

B. 8 


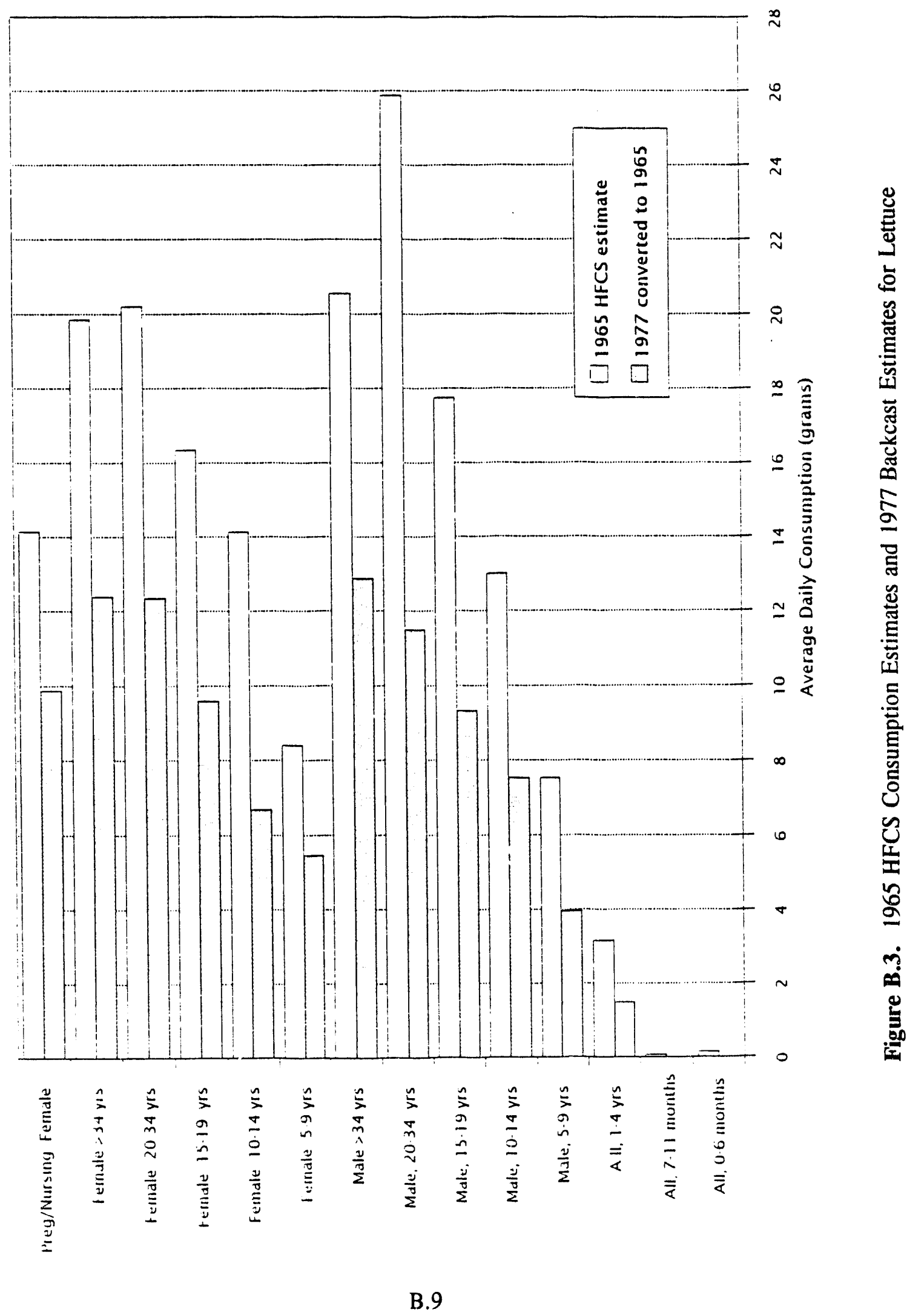




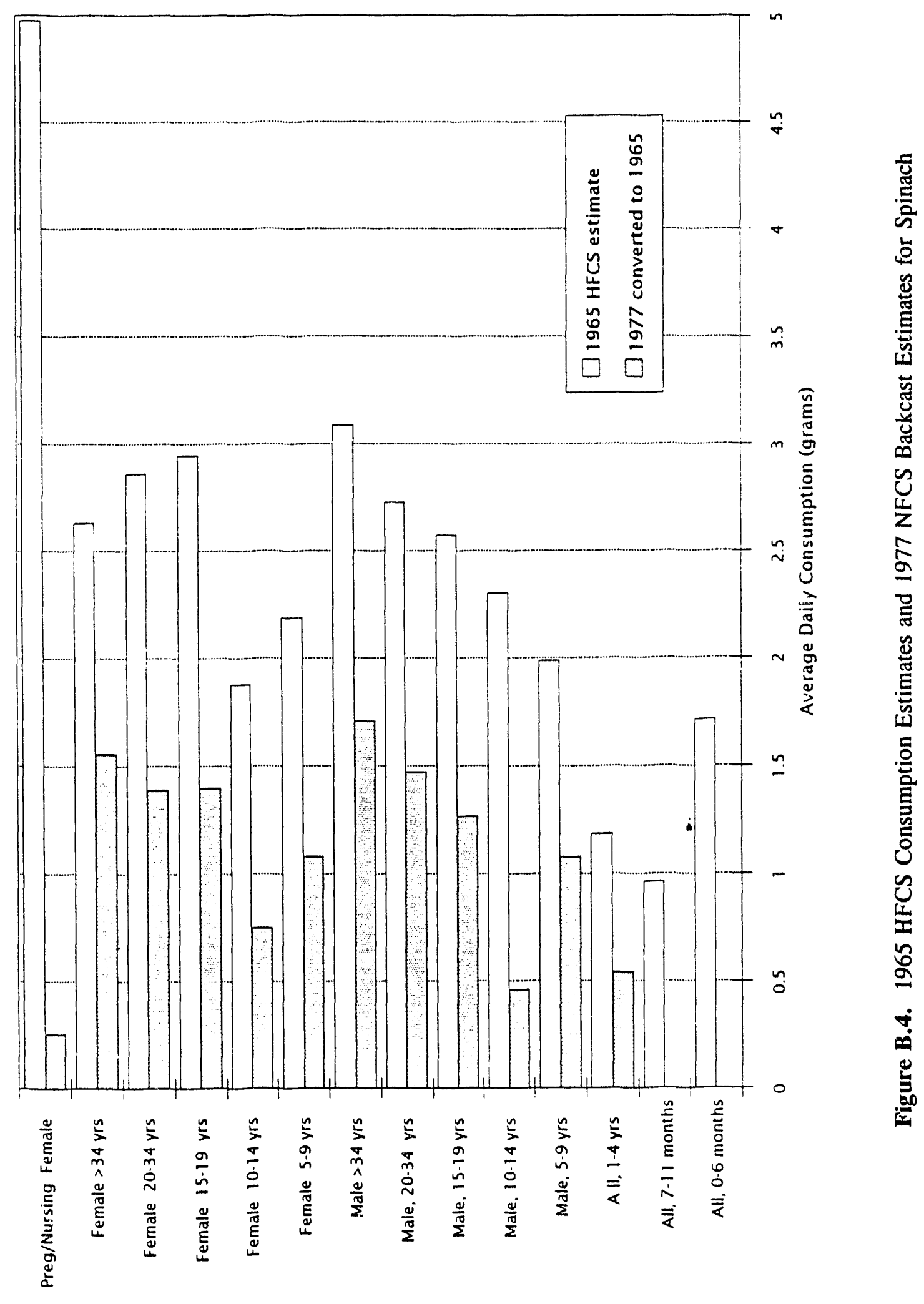

B. 10 


\section{Comparison With Other Estimates}

Table B. 2 shows a comparison of per capita consumption derived from the consumption values of the several studies listed. Milk and eggs were the only foods defined well enough in all studies to use for comparison. Daily per capita consumption values were the only compatible units of measure to use for comparison and still be able to compare backcasts to representative rural consumption data. The backcasting method seems to consistently underestimate rural consumption. The 1950, 1954, and 1969 rural studies (Bustad and Terry 1956; USDA 1955b; Shipler et al. 1972) consistently estimated per capita egg consumption over 50 percent higher on average than the backcasting method. On average, per capita milk consumption was backcast over 15 percent lower than the rural studies. Backcasting performed well when compared with per capita estimates from the 1965 HFCS (USDA 1972), which included urban and rural classifications.

The backcasting method's inability to predict rural consumption could be offset by applying a rural adjustment factor, especially to obtain a more reliable estimate for milk. That factor should probably be at least 15 percent to fuily offset underestimation of rural milk consumption. There is not enough information to determine the implications of underestimating rural consumption within specific age/sex groups. There are no compatible local data for any group other than children ages 5-14. That comparison is shown in Table 2.1 of this report and indicates that backcasting performed better when the projection period was shorter and specific age groups were compared. It was not unexpected that the backcasting method's ability to predict consumption would decrease as the projection horizon lengthens.

Table B.2. Comparison of Local and National Average Daily Per Capita Consumption of Milk and Eggs to Estimates Backcasted from NFCS Data.

\begin{tabular}{|l|l|c|c|c|c|c|c|}
\hline & Milk & Eggs & $\begin{array}{c}\text { Backcast } \\
\text { Estirnate } \\
\text { for Milk } \\
\text { Year }\end{array}$ & \multicolumn{1}{|c|}{ Data Source } & $\begin{array}{c}\text { Backcast } \\
\text { Estimate } \\
\text { for Eggs } \\
\text { (g/day) }\end{array}$ & $\begin{array}{c}\text { Difference } \\
\text { in Milk } \\
\text { Backcast } \\
\text { (percent) }\end{array}$ & $\begin{array}{c}\text { Difference } \\
\text { in Eggs } \\
\text { Backcast } \\
\text { (percent) }\end{array}$ \\
\hline 1950 & Bustad and Terry (1956) & 870 & 81.5 & 536 & 29.0 & -38.4 & -64.4 \\
1954 & USDA (1955b) & 470 & 46.2 & 550 & 28.2 & +17.0 & -40.0 \\
& (Rural households) & & & & & & -25.0 \\
1969 & Shipler et al. (1972) & 580 & 52.5 & 435 & 23.4 & -55.4 \\
1965 & 1965 HFCS (USDA 1972) & 495 & 23.4 & 485 & 23.7 & -0.02 & +0.01 \\
\hline
\end{tabular}




\section{Appendix C}

Summary Consumption Statistics 


\section{Appendix C}

\section{Summary Consumption Statistics}

This appendix contains the summary consumption distribution statistics for the food groups analyzed for this report (Tables C.1-C.59), except those presented in the body of the report. The statistics were calculated from the raw responses to the 1977-1978 NFCS and do not account for differences in consumption by season. These tables were derived from the same NFCS subsample as those in the body of the report. All "central city" responses were excluded ac were those from the regions located in the South.

Table C.60 provides the food groups associated with the column headings in Tables C.61-C.63. The estimated average daily consumption (grams) in 1945, 1951, and 1957 for each food has been included in Tables C.61-C.63. The means presented are calculated for days when consumption occurred and represent mean consumption per day. These means are higher than overall means (means that are calculated to include days with no consumption). Tables C.64-C.66 provide the estimated average daily consumption for those foods that are in the DID. 


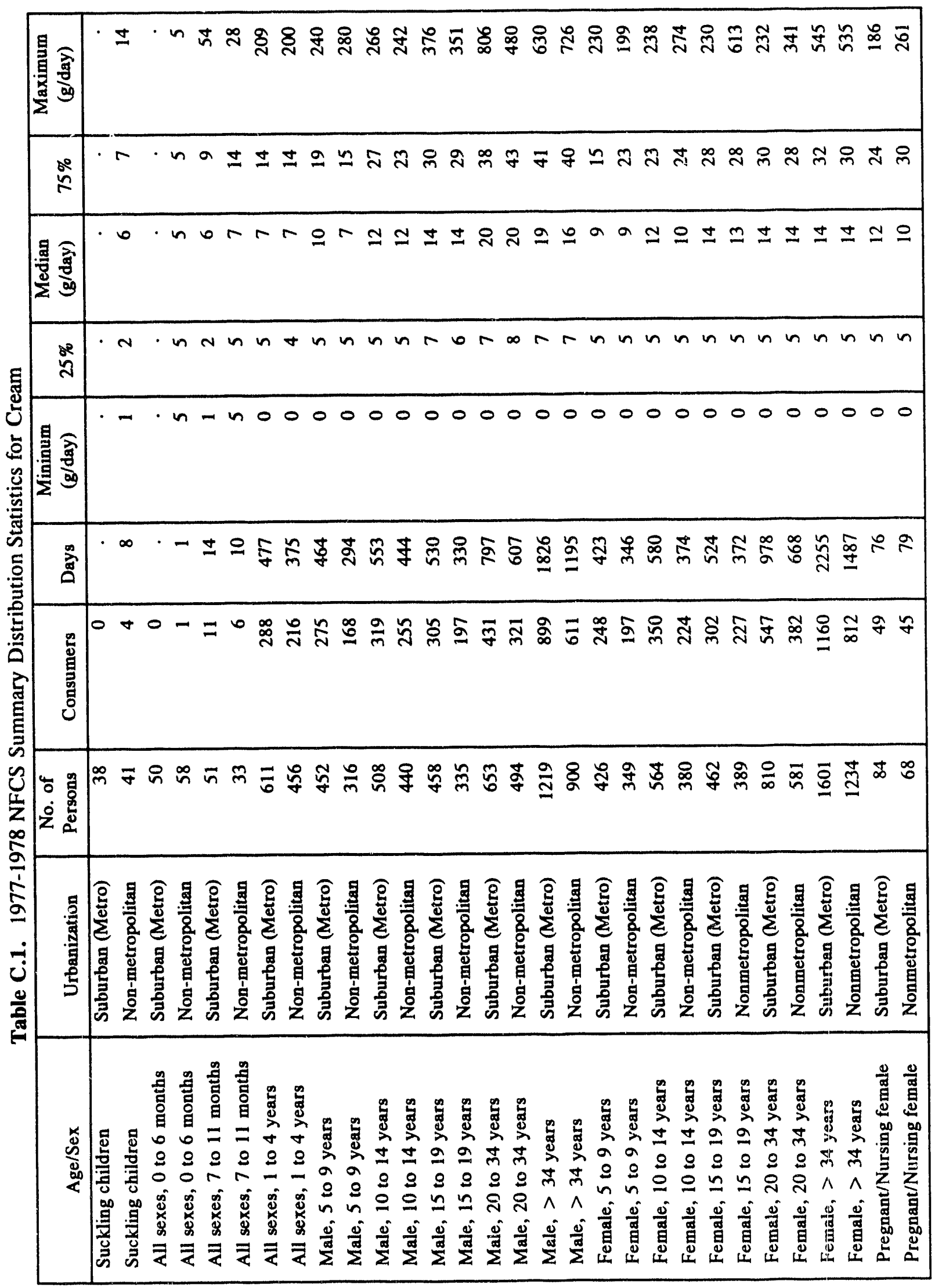

C. 2 


\begin{tabular}{|c|c|}
\hline 量 & 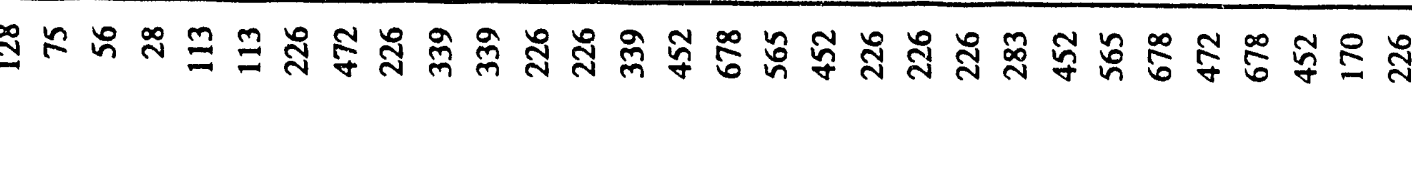 \\
\hline مُ & 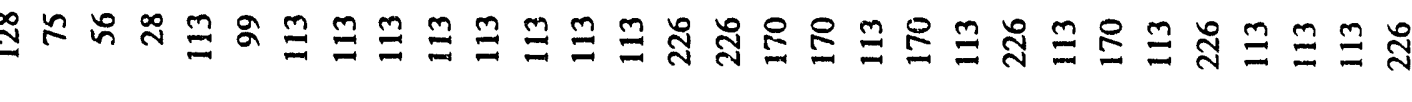 \\
\hline $\mid$ & 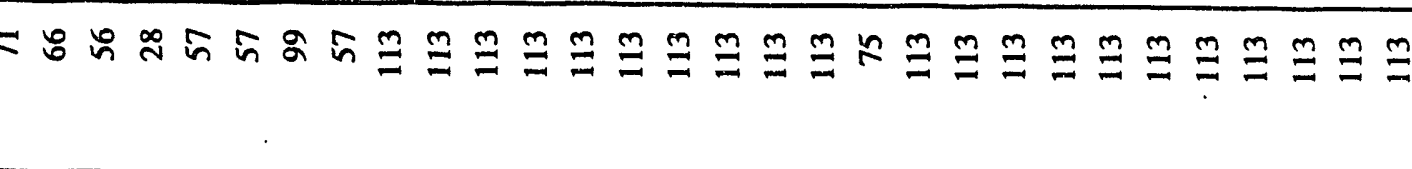 \\
\hline 然 & 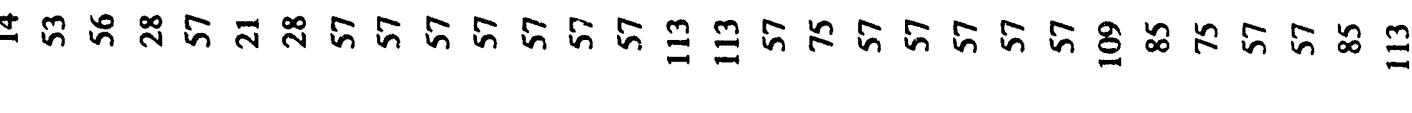 \\
\hline 量 & 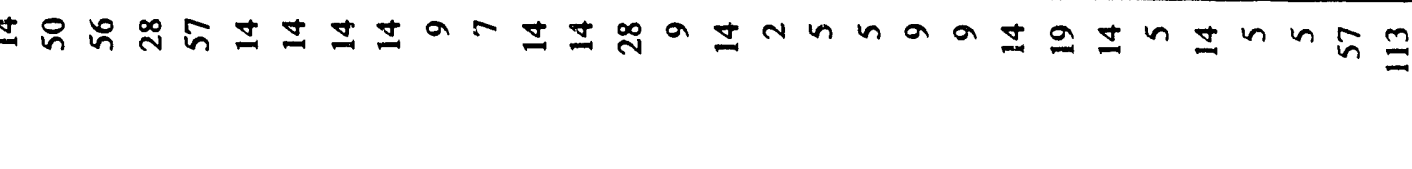 \\
\hline 咅 & 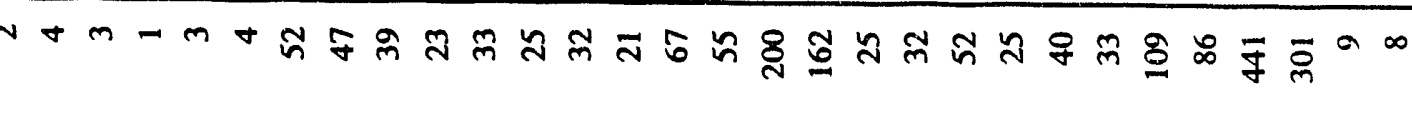 \\
\hline | & 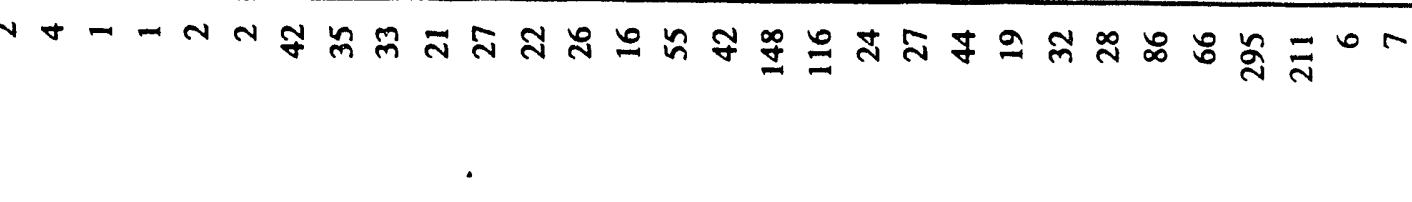 \\
\hline 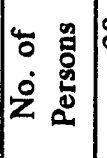 & 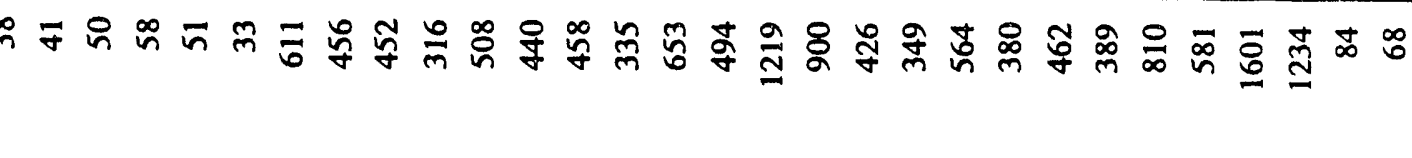 \\
\hline & 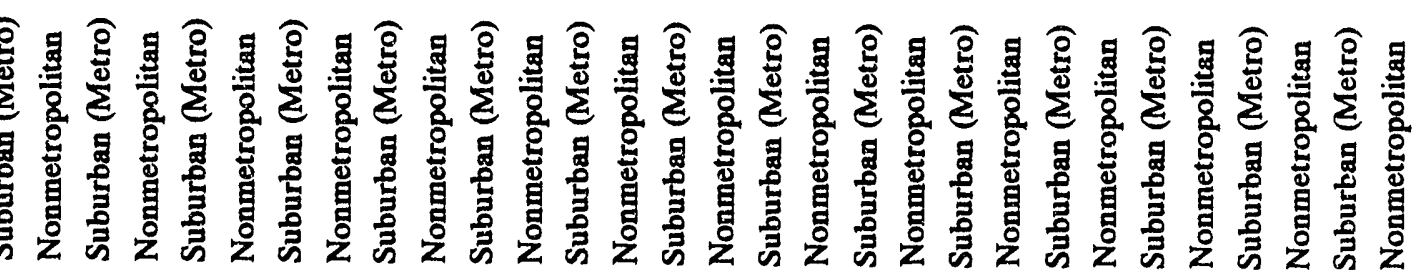 \\
\hline & 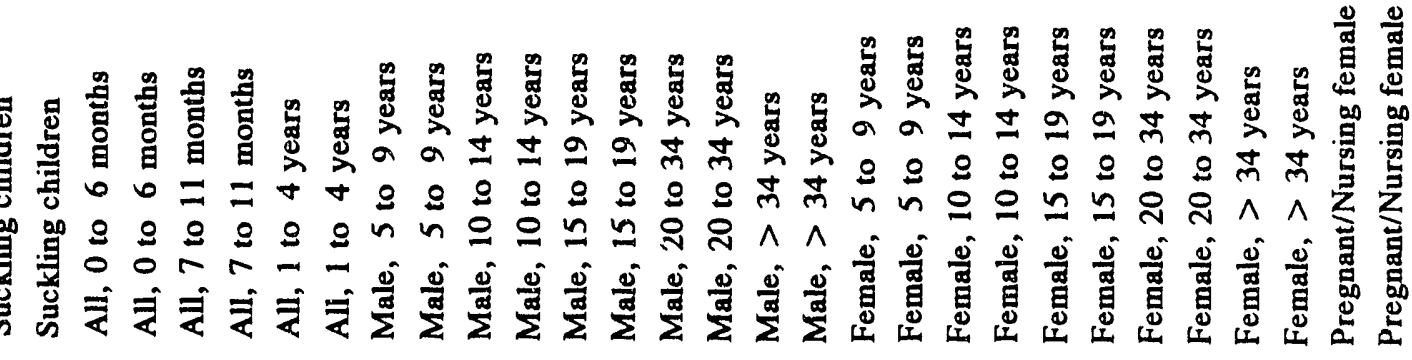 \\
\hline
\end{tabular}

C. 3 


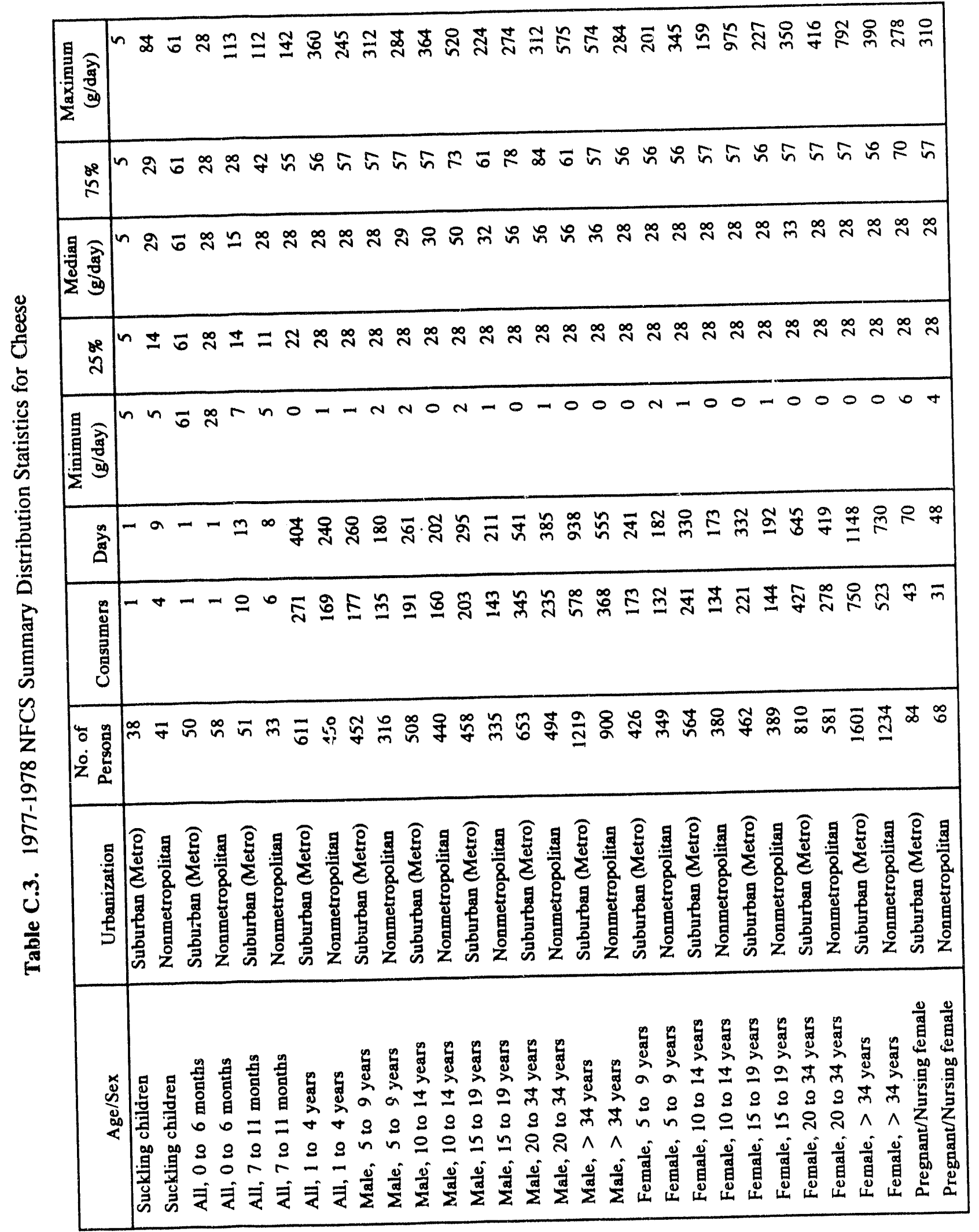

C. 4 


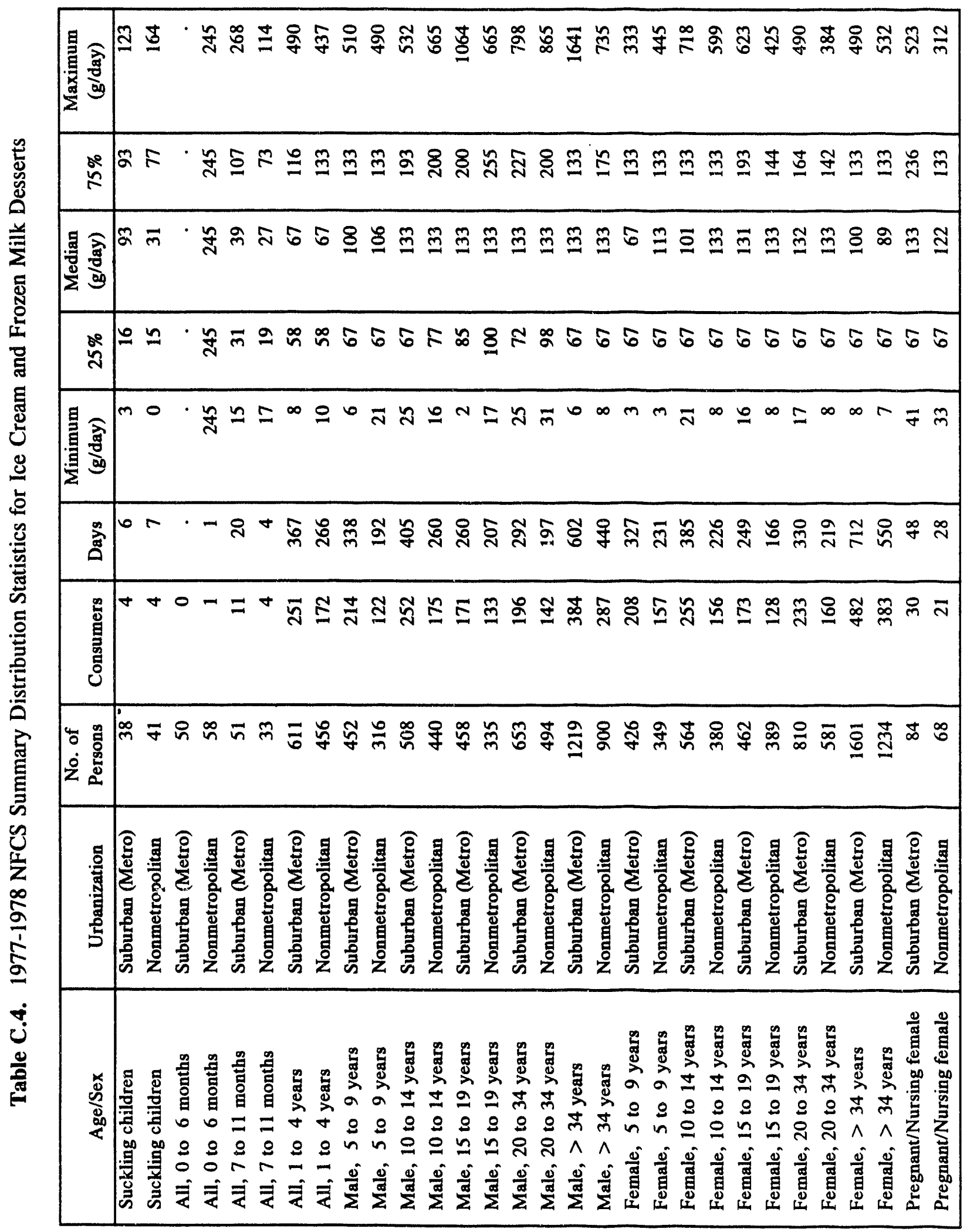




\begin{tabular}{|c|c|}
\hline 量 & 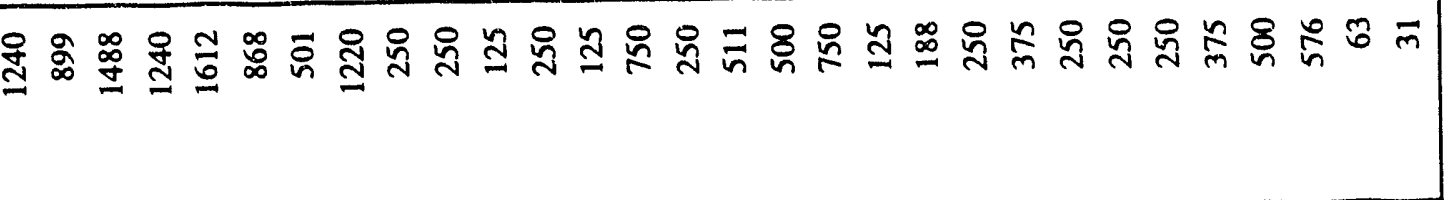 \\
\hline$\stackrel{8}{8}$ & 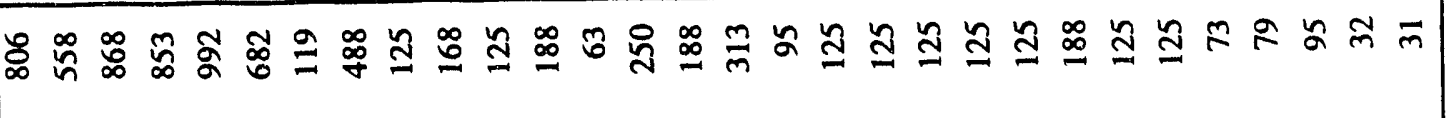 \\
\hline 蛋 & 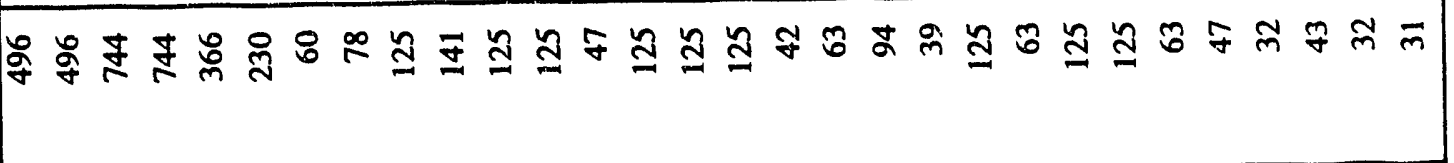 \\
\hline ڤe & 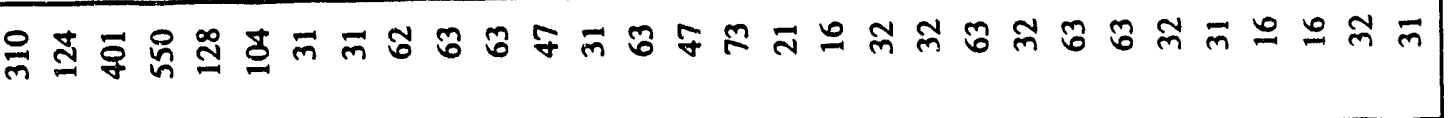 \\
\hline$\frac{1}{2}$ & 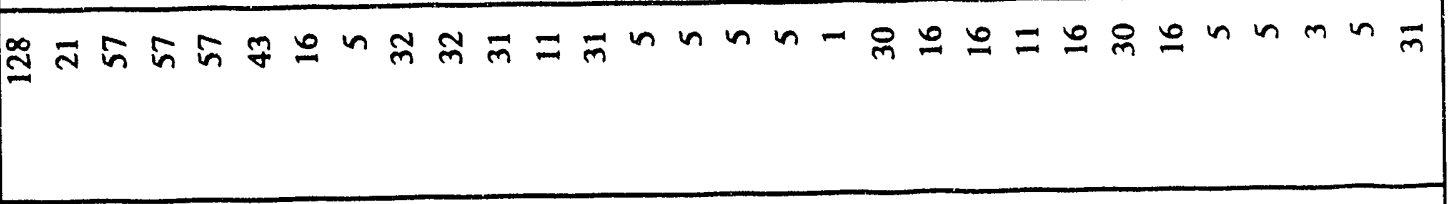 \\
\hline 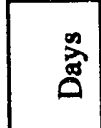 & 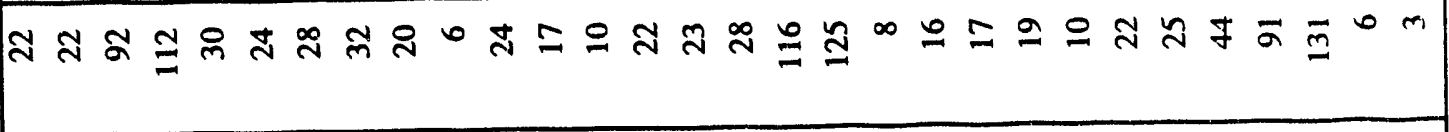 \\
\hline 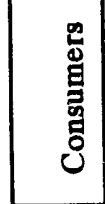 & 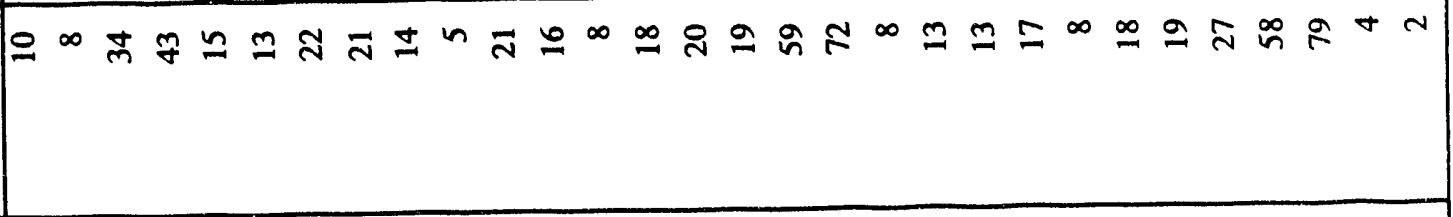 \\
\hline 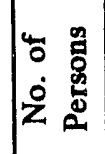 & 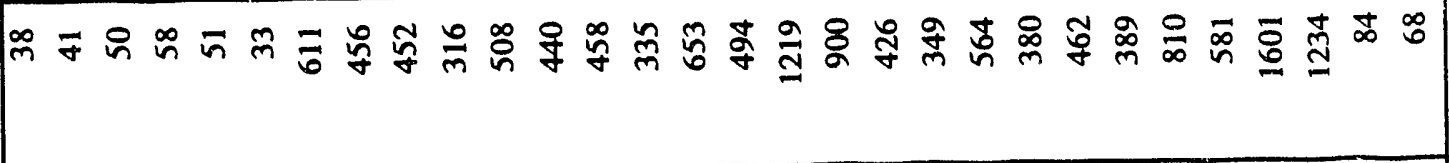 \\
\hline 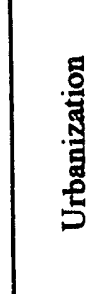 & 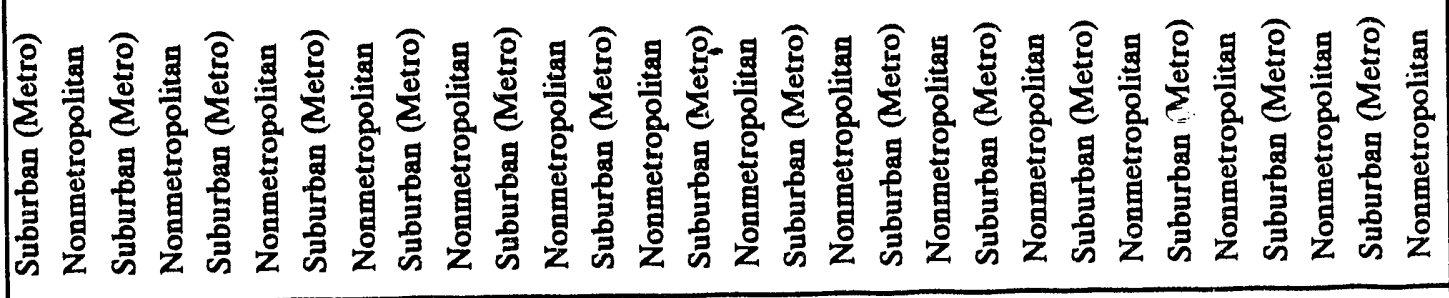 \\
\hline & 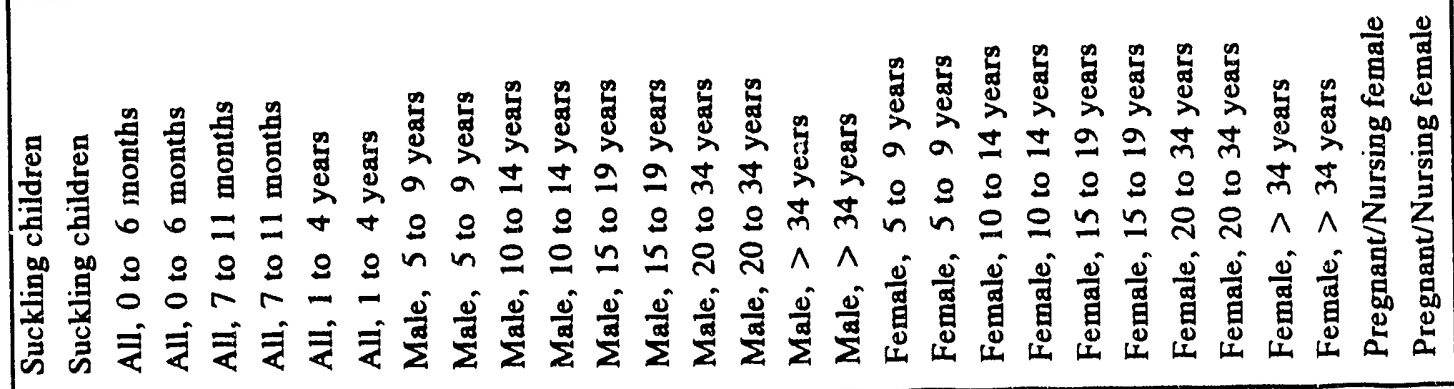 \\
\hline
\end{tabular}




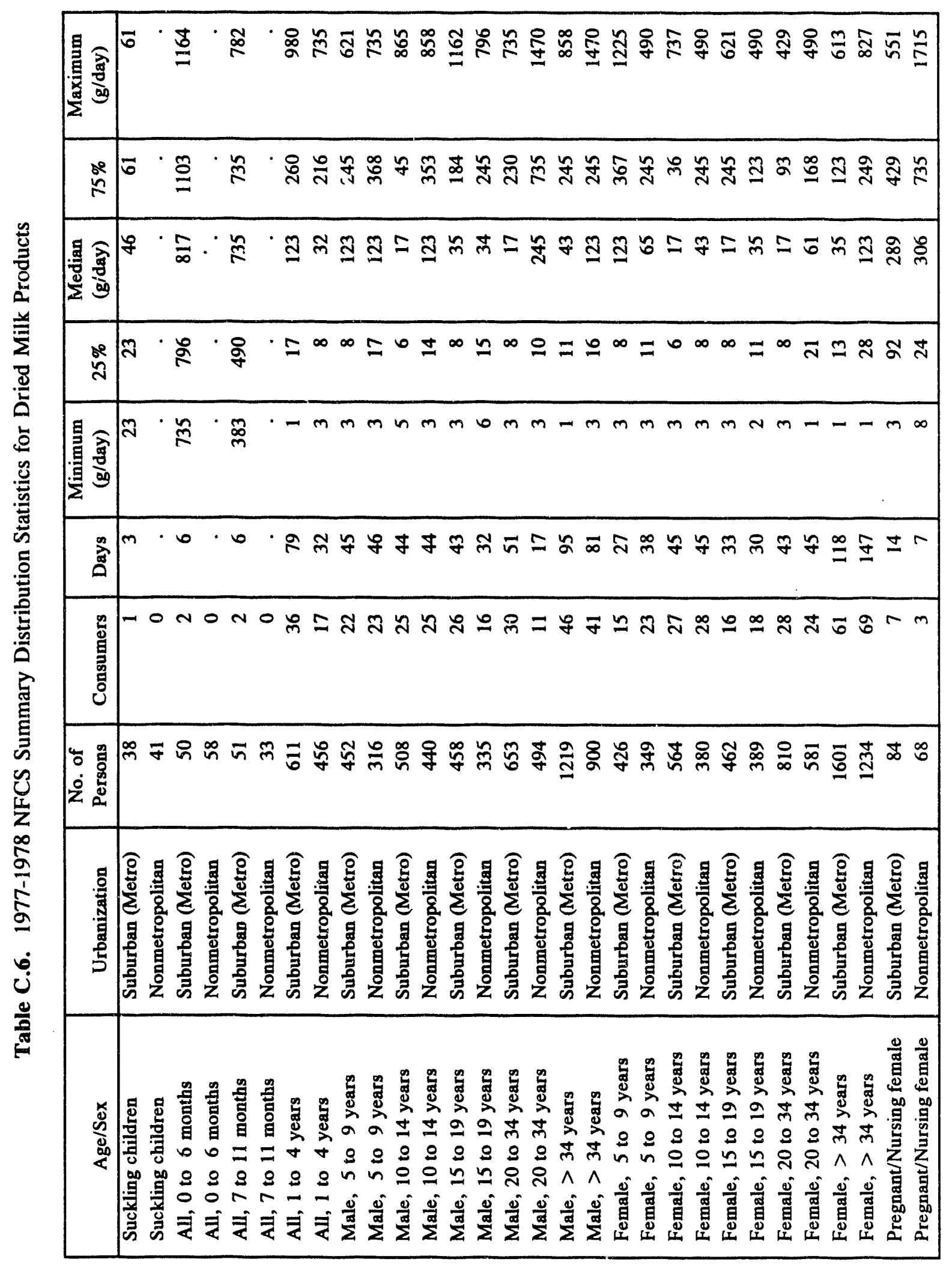

C. 7 


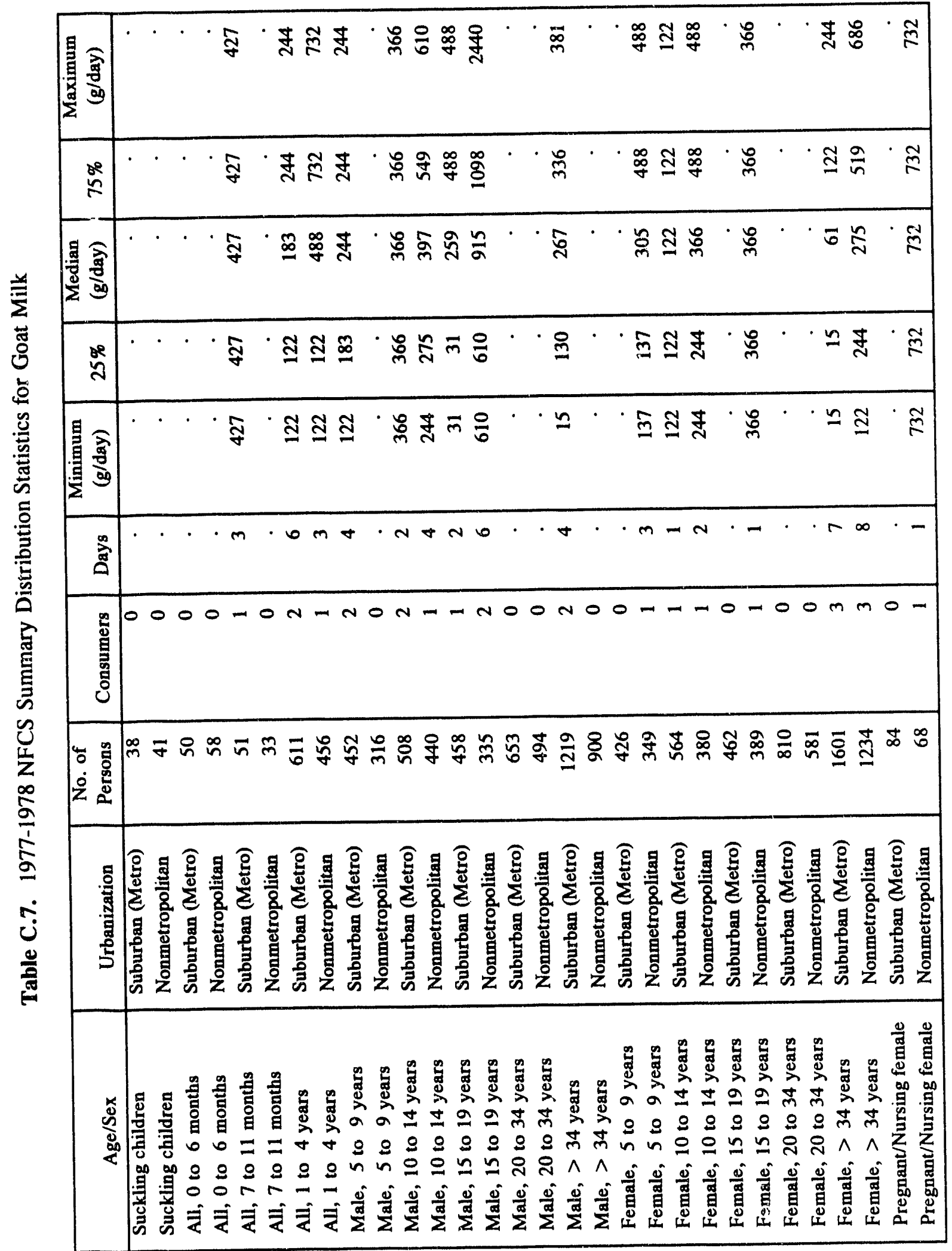

C. 8 


\begin{tabular}{|c|c|}
\hline 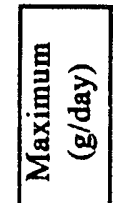 & 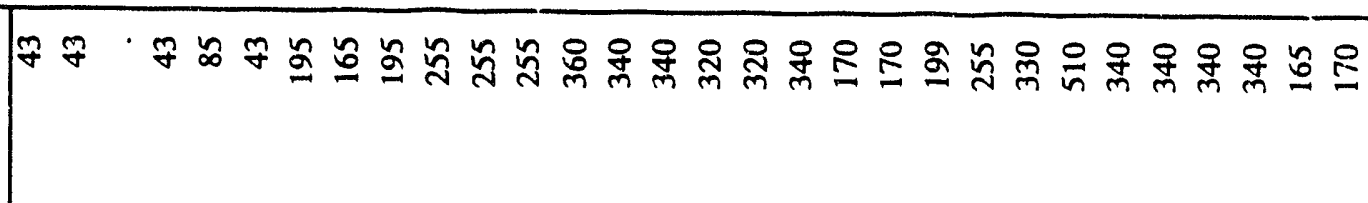 \\
\hline 今. & 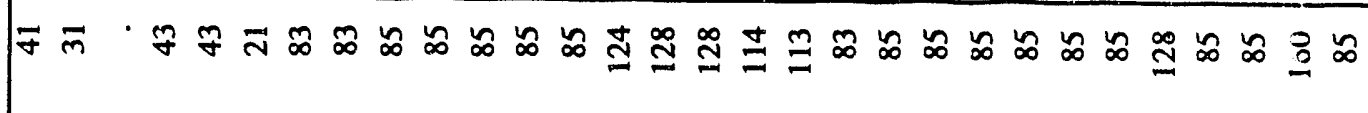 \\
\hline 墨 & 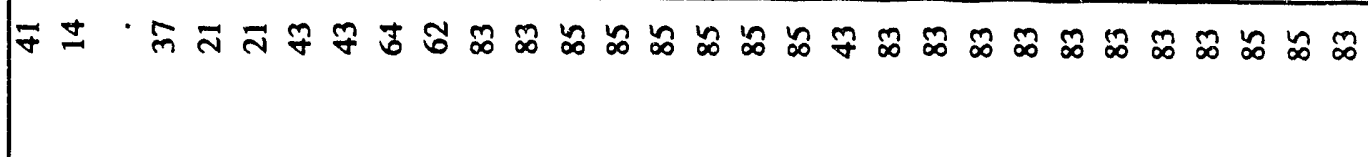 \\
\hline$\stackrel{x}{a}$ & 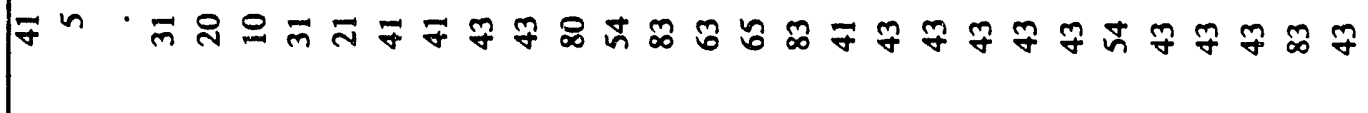 \\
\hline 量 & 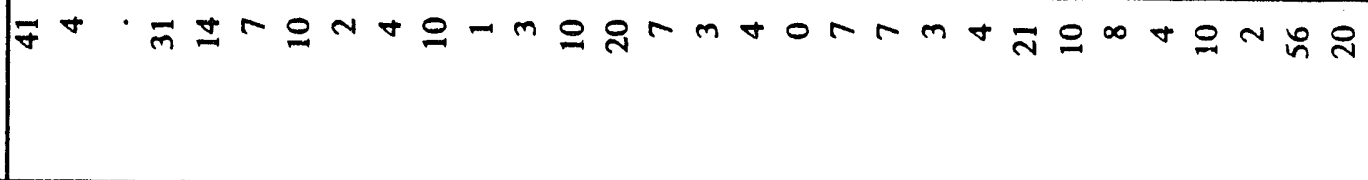 \\
\hline 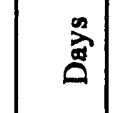 & 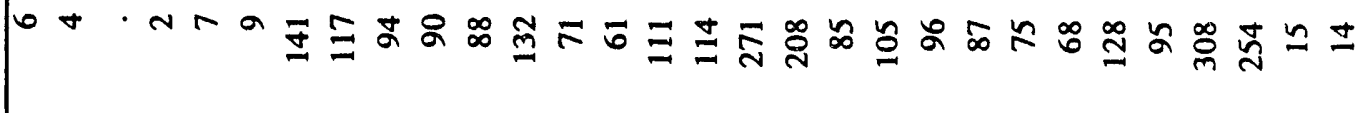 \\
\hline 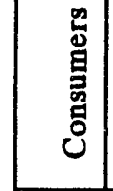 & 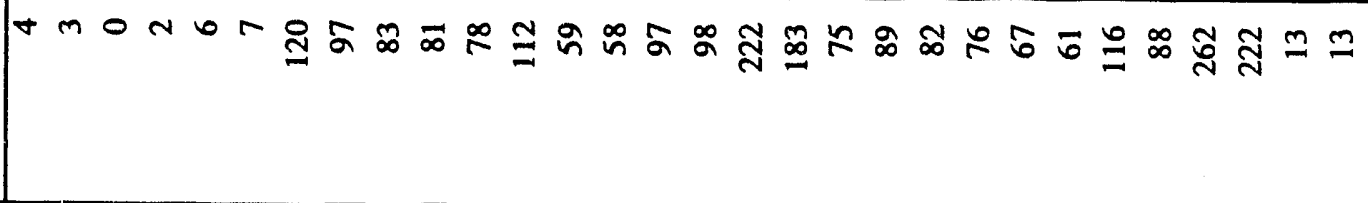 \\
\hline 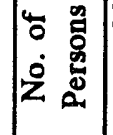 & 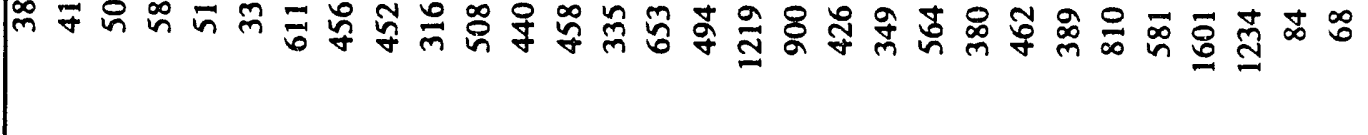 \\
\hline & 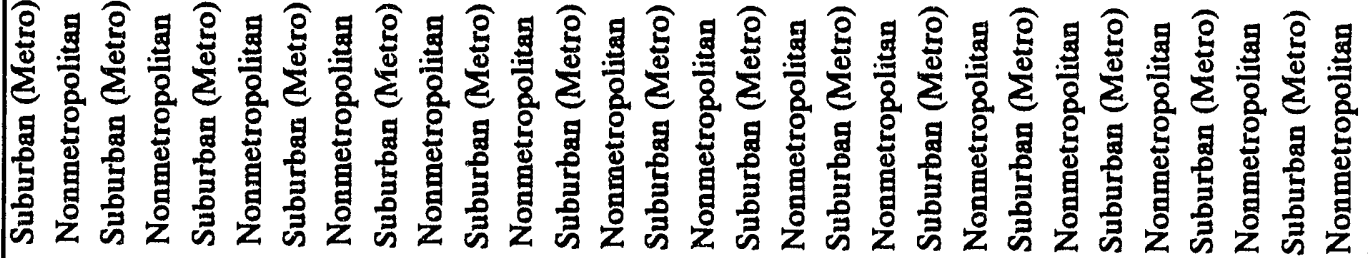 \\
\hline & 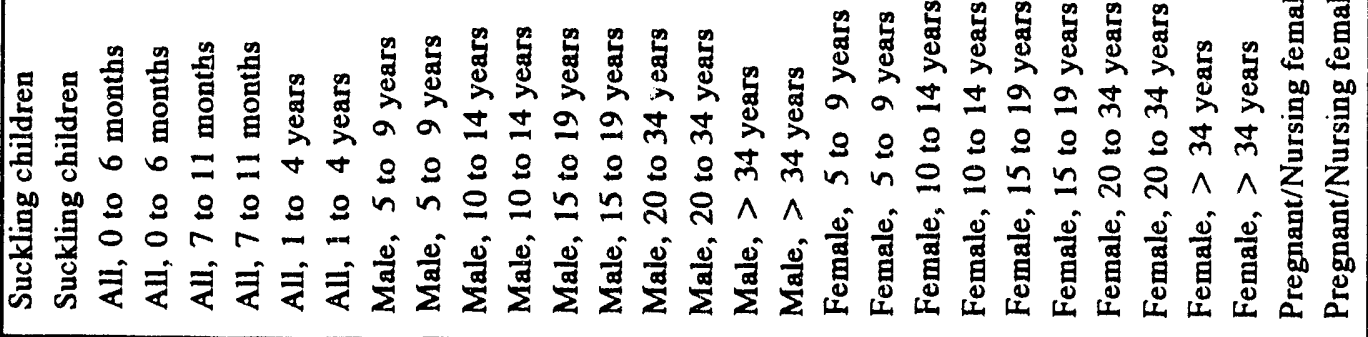 \\
\hline
\end{tabular}

C. 9 


\begin{tabular}{|c|c|}
\hline 量 & 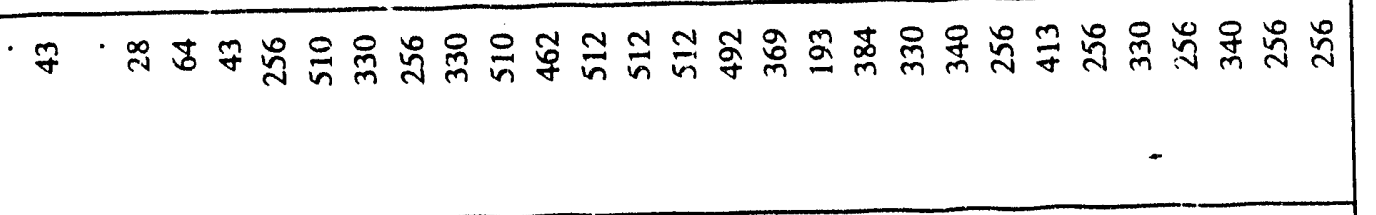 \\
\hline$\stackrel{8}{i}$ & 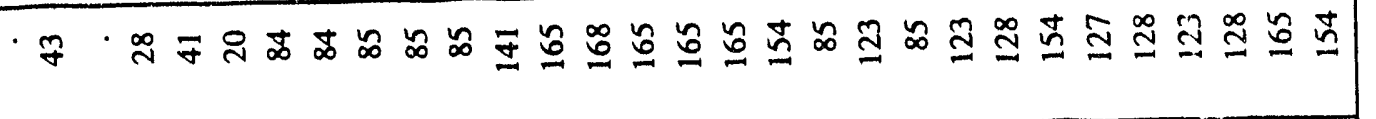 \\
\hline$\frac{\sqrt{3}}{3}$ & 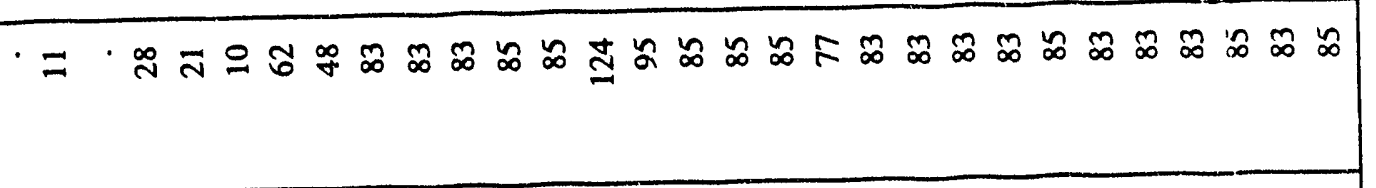 \\
\hline$y^{\circ}$ & 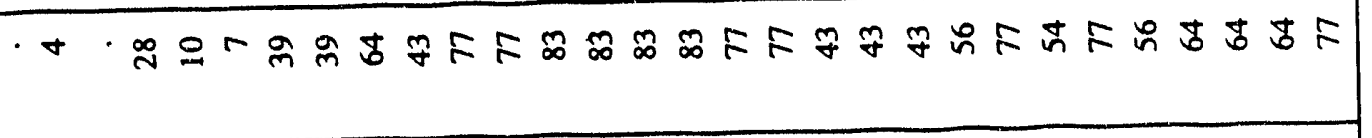 \\
\hline$\frac{1}{3}$ & 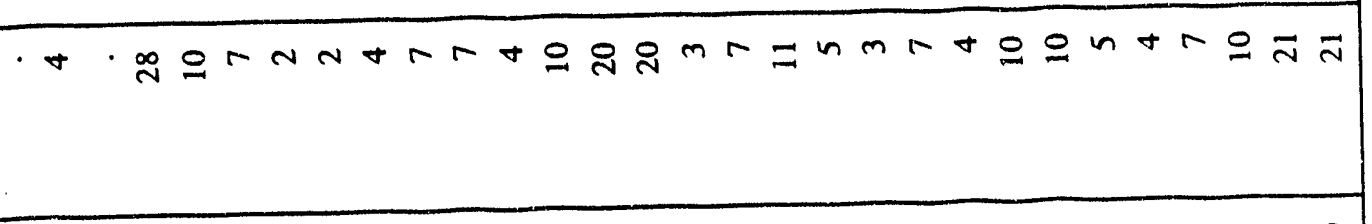 \\
\hline 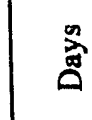 & 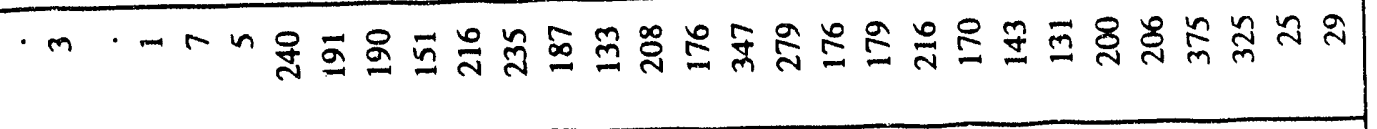 \\
\hline 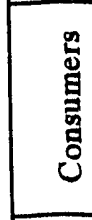 & 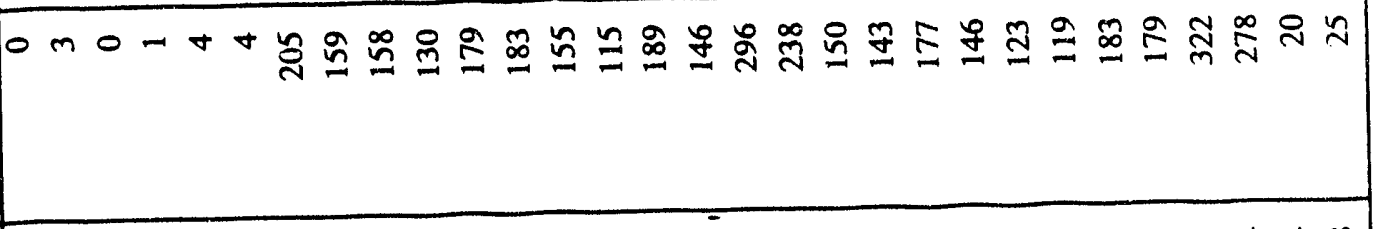 \\
\hline 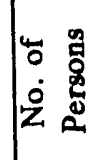 & 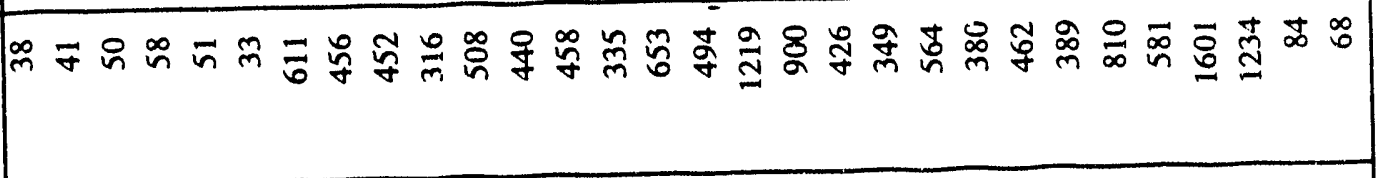 \\
\hline $\begin{array}{l}\text { 蛋 } \\
\text { 点 }\end{array}$ & 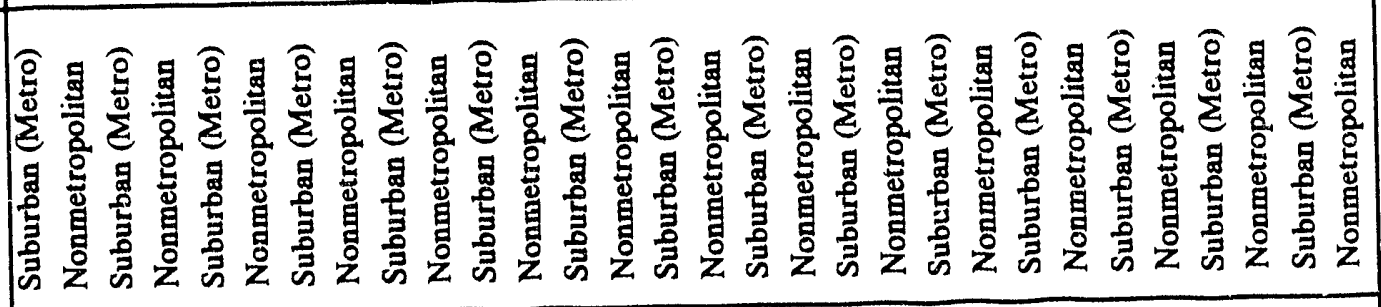 \\
\hline & 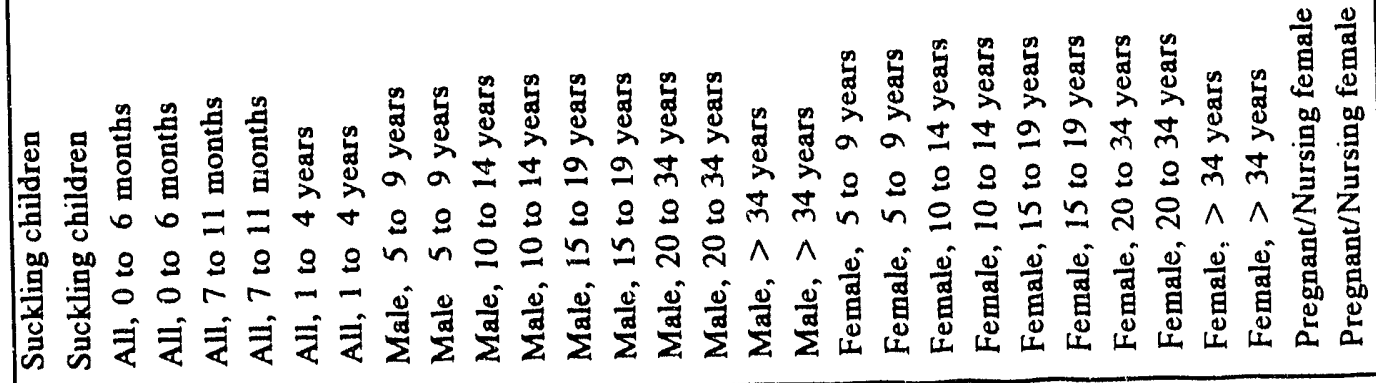 \\
\hline
\end{tabular}

C. 10 


\begin{tabular}{|c|c|}
\hline 量要 & 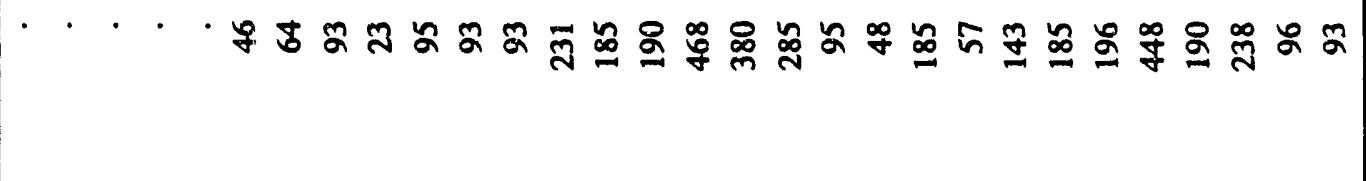 \\
\hline$\stackrel{8}{8}$ & 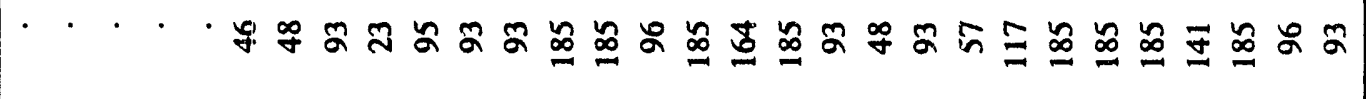 \\
\hline 透 & 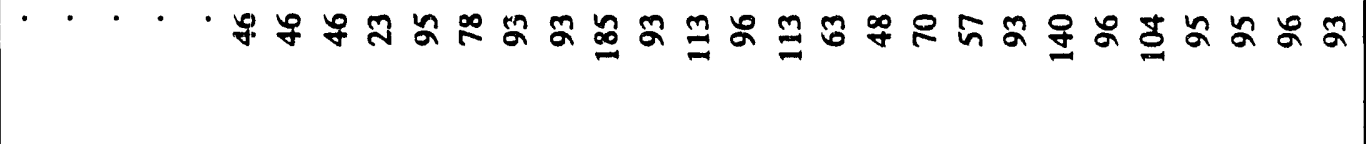 \\
\hline$\ddot{z}$ & 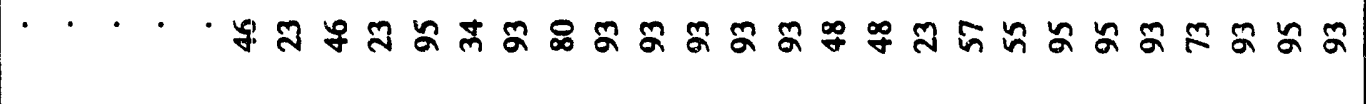 \\
\hline 量 & 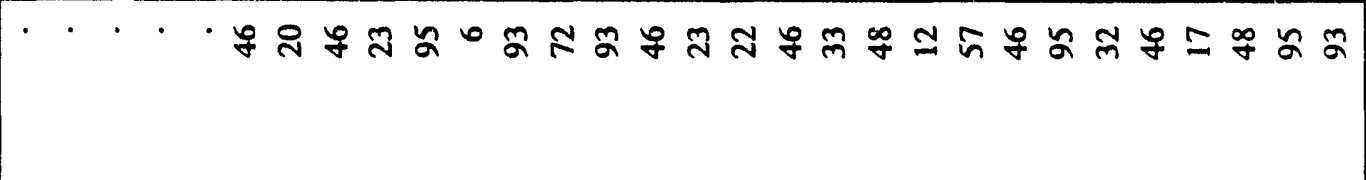 \\
\hline$\stackrel{\infty}{a}$ & 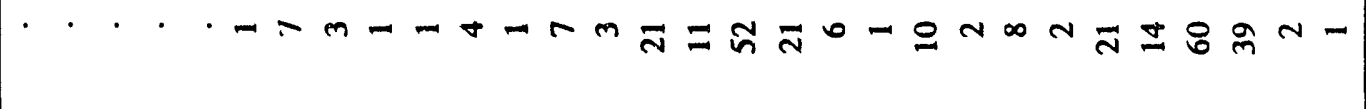 \\
\hline 量 & 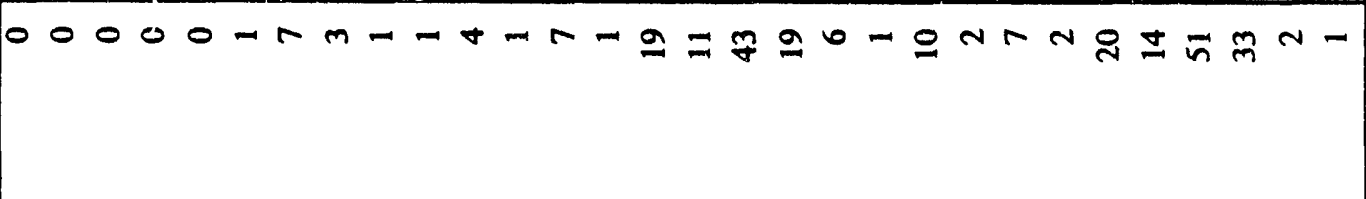 \\
\hline 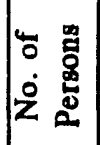 & 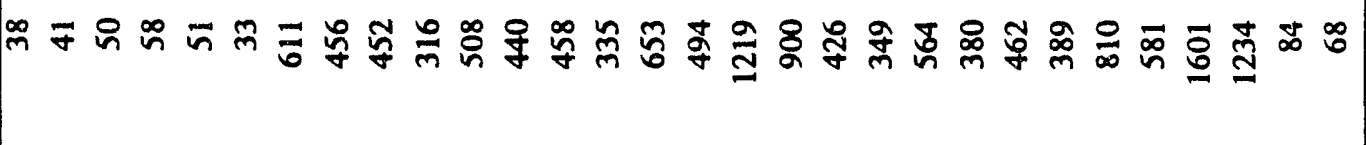 \\
\hline 兽 & 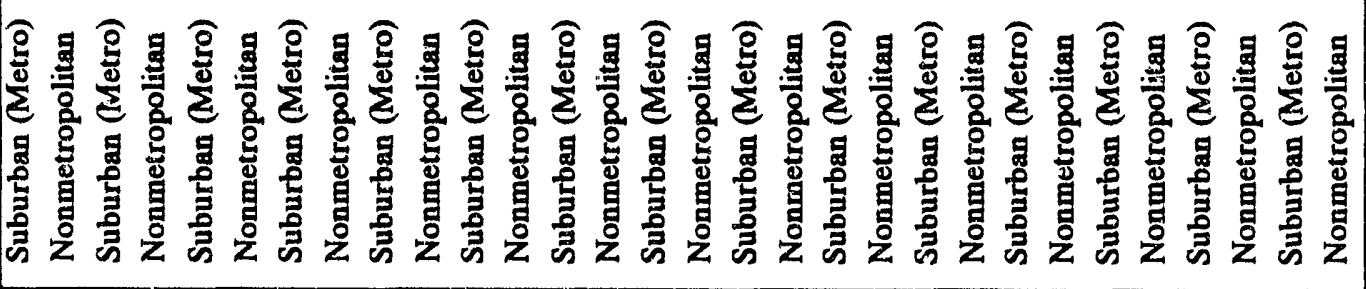 \\
\hline . & 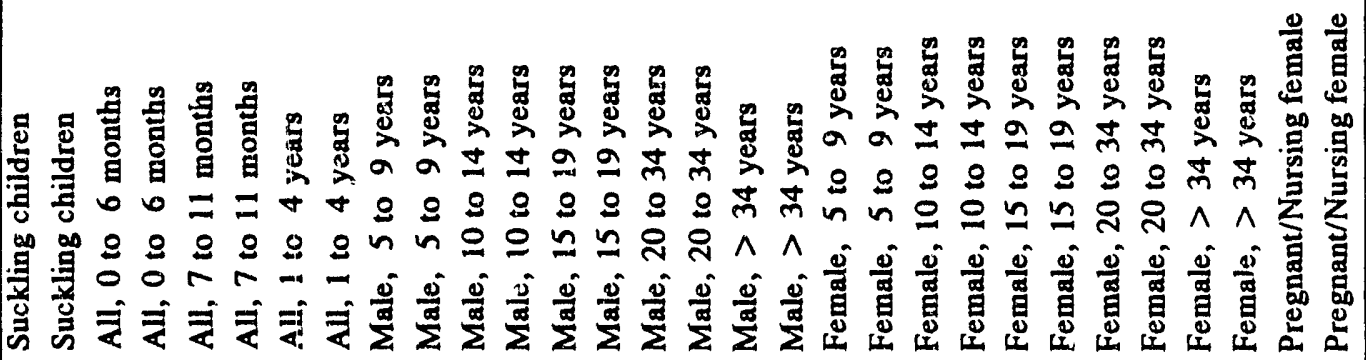 \\
\hline
\end{tabular}

C. 11 


\begin{tabular}{|c|c|}
\hline 拿 & 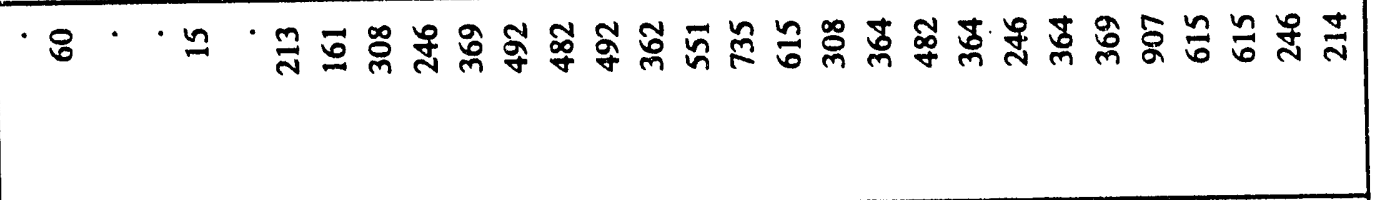 \\
\hline so & 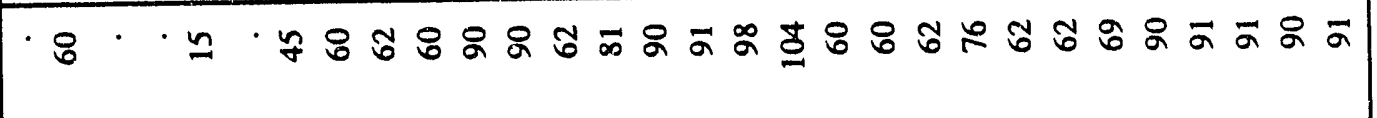 \\
\hline 焉 & 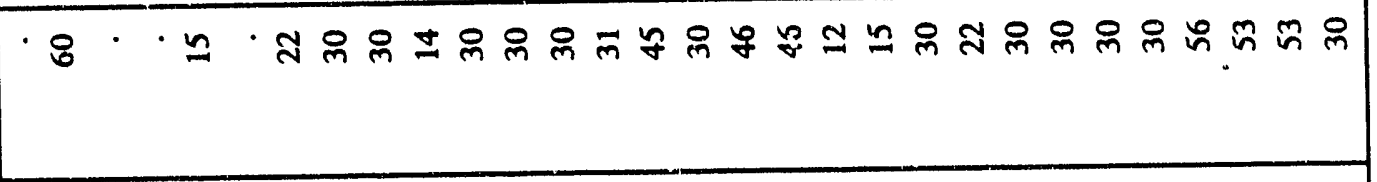 \\
\hline$\ddot{y}$ & 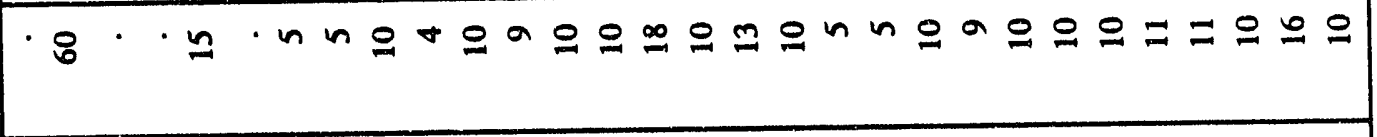 \\
\hline$\frac{1}{3}$ & $B \cdot g \cdot 0-n-m-t m m m m-n-n-n h n d a m$ \\
\hline$\stackrel{\infty}{\stackrel{\Delta}{\Delta}}$ & 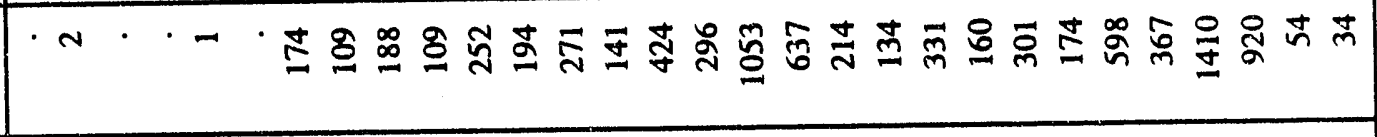 \\
\hline 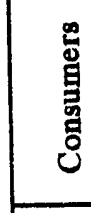 & 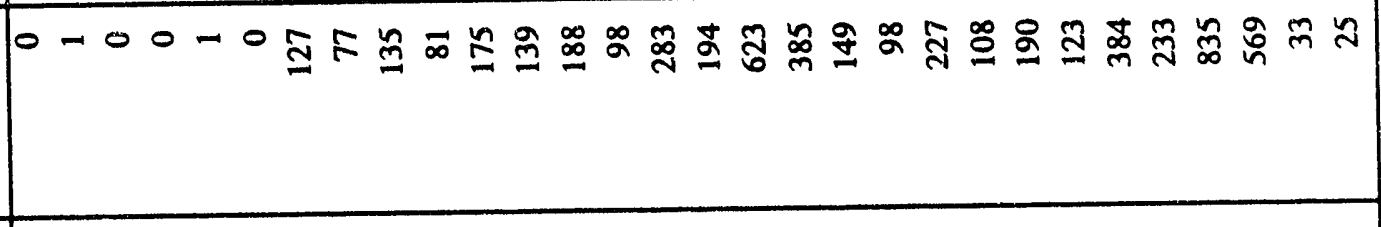 \\
\hline 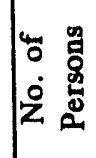 & 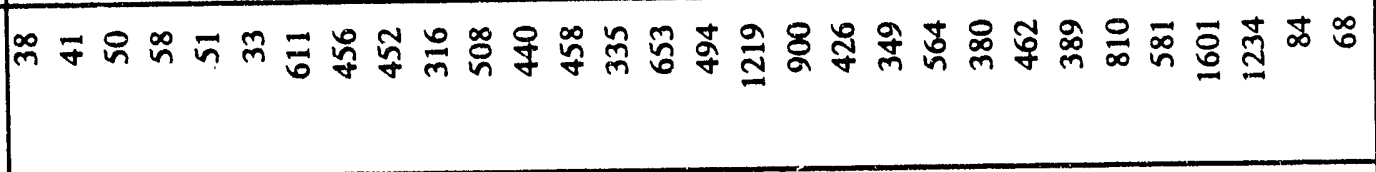 \\
\hline 至 & 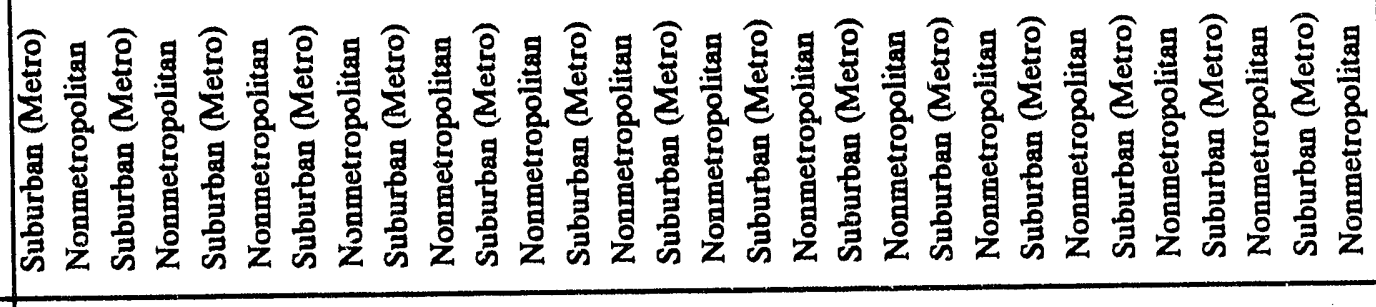 \\
\hline & 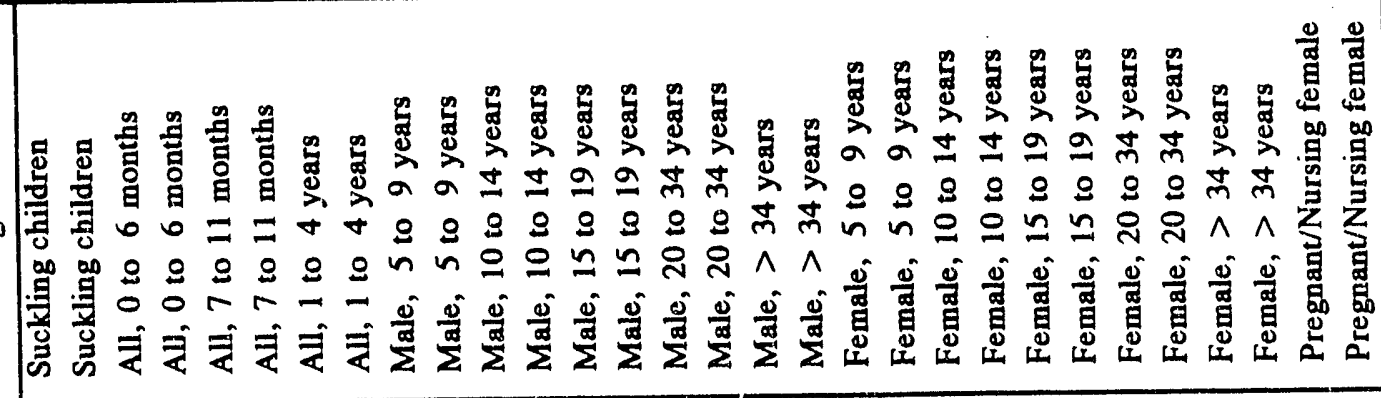 \\
\hline
\end{tabular}

C. 12 


\begin{tabular}{|c|c|}
\hline 量 & 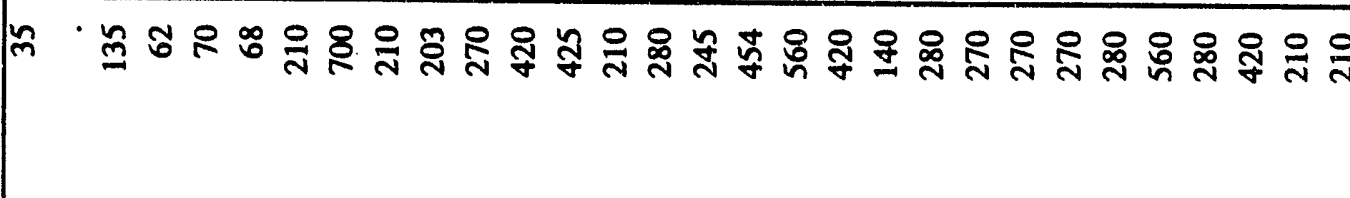 \\
\hline$\stackrel{s}{*}$ & 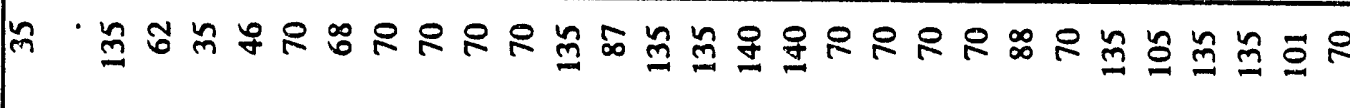 \\
\hline 通 & 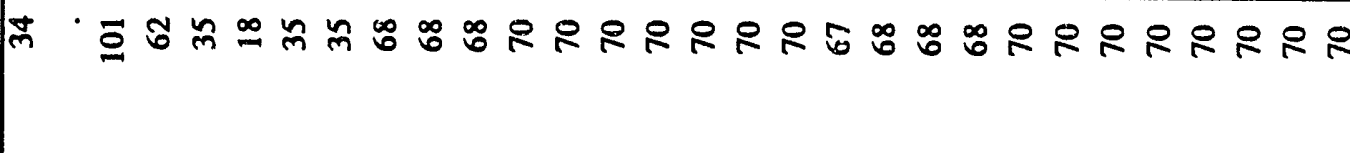 \\
\hline 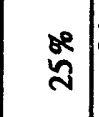 & 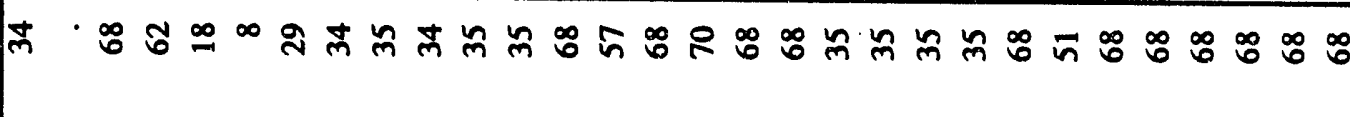 \\
\hline 量要 & स. \\
\hline 离 & 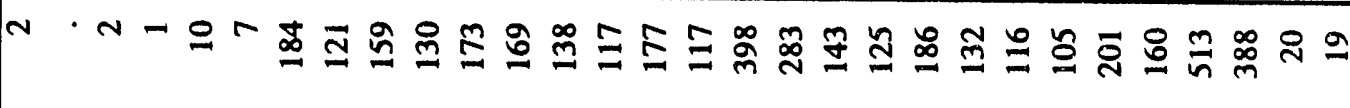 \\
\hline $\begin{array}{l}\text { : } \\
\text { 兽 } \\
\text { نे }\end{array}$ & NOー- \\
\hline 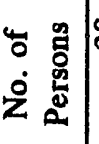 & 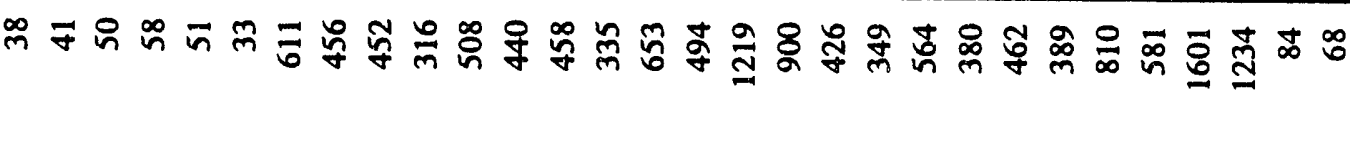 \\
\hline & 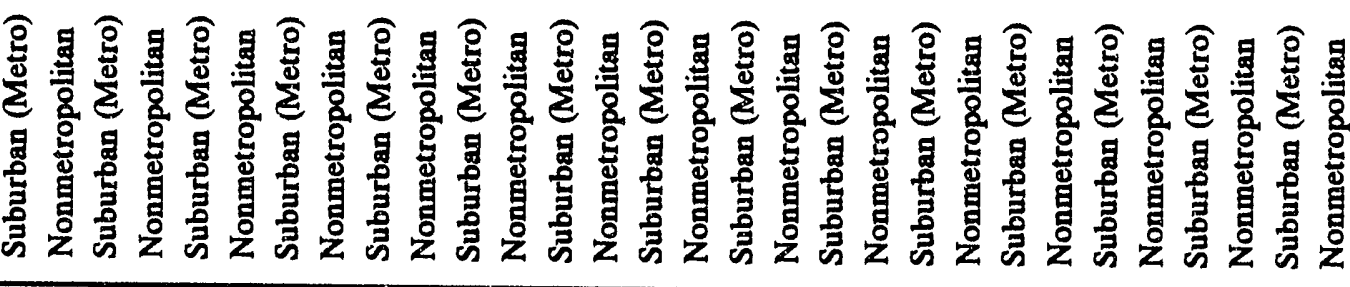 \\
\hline 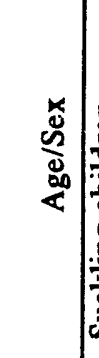 & 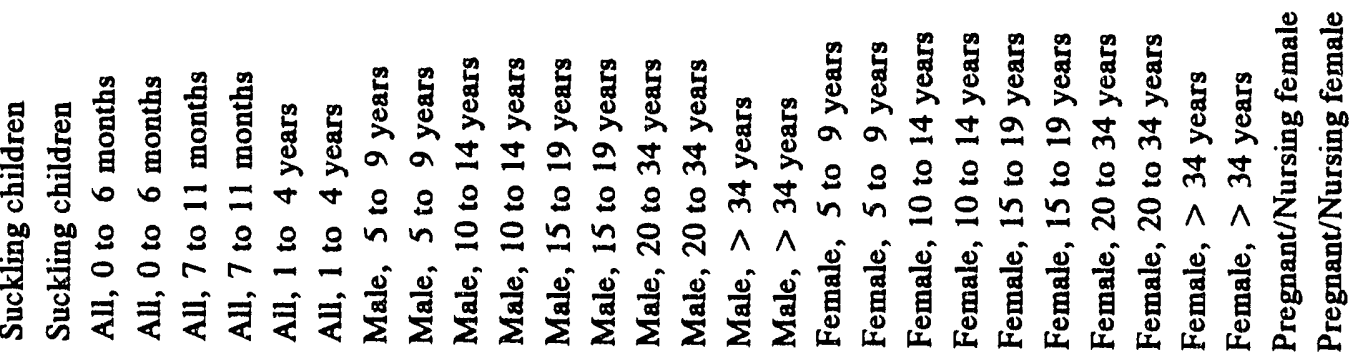 \\
\hline
\end{tabular}

C. 13 


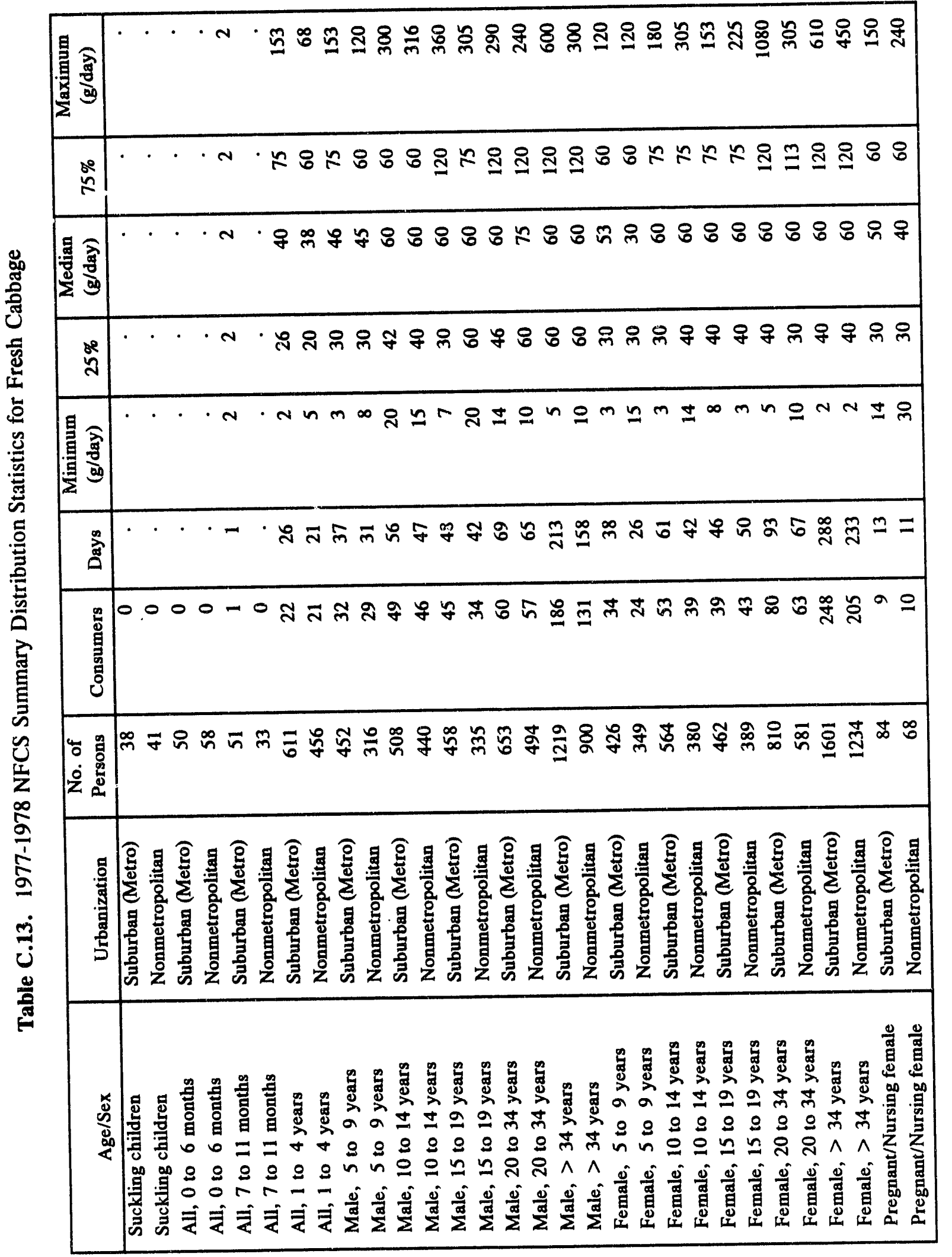

C. 14 


\begin{tabular}{|c|c|}
\hline 量 & 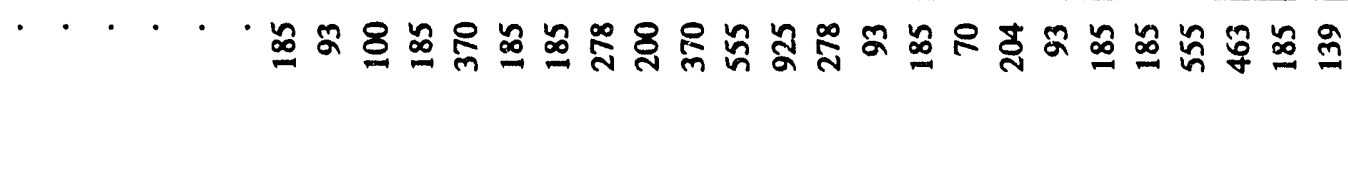 \\
\hline \multirow{3}{*}{$\mid \frac{3}{2}$} & 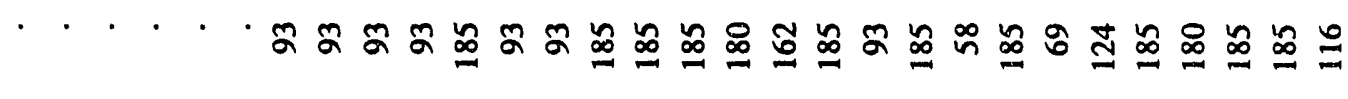 \\
\hline & 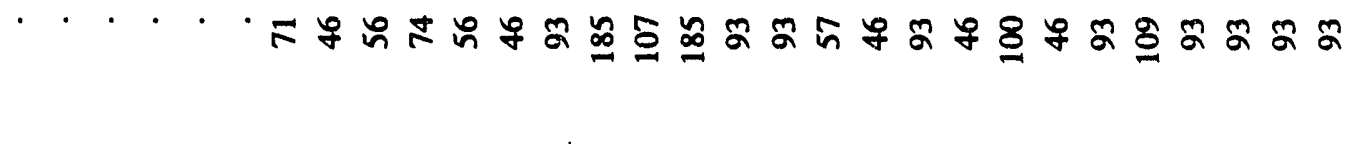 \\
\hline & 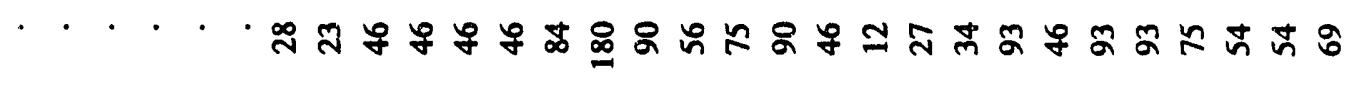 \\
\hline 高 & …… \\
\hline$\stackrel{n}{9}$ & 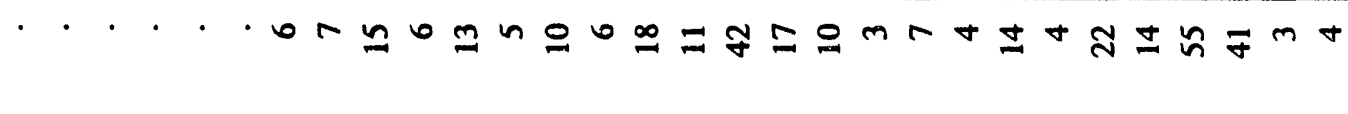 \\
\hline 畩 & 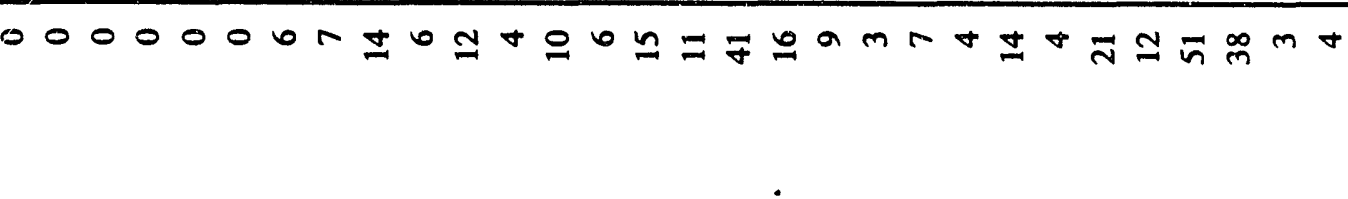 \\
\hline 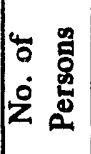 & 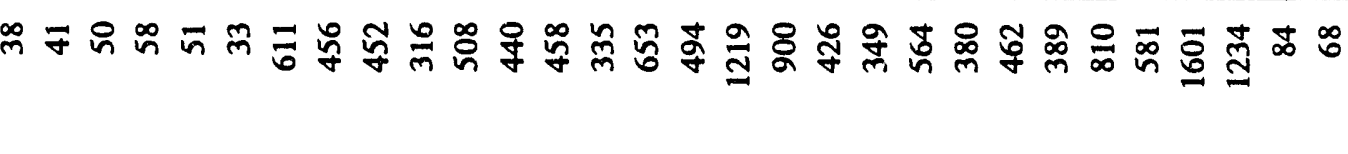 \\
\hline 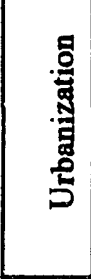 & 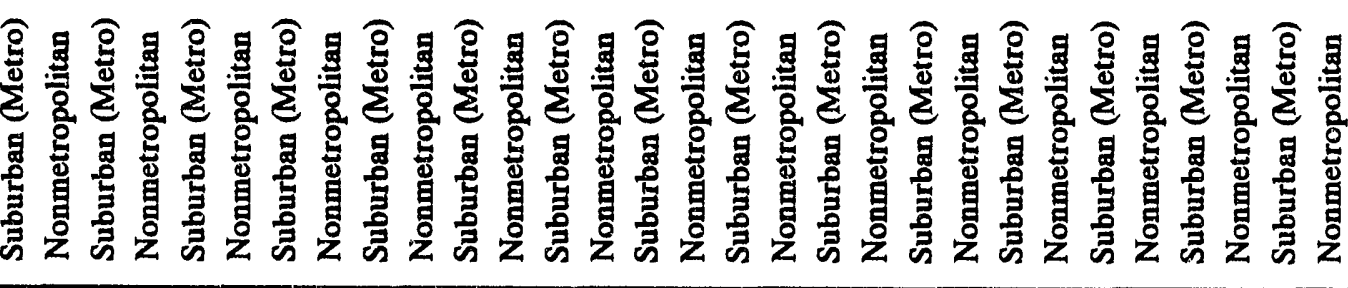 \\
\hline 赵 & 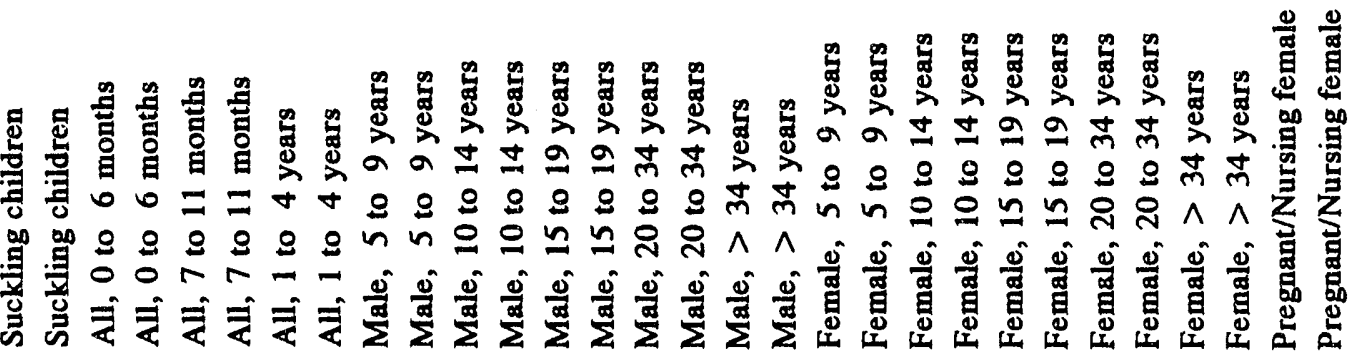 \\
\hline
\end{tabular}

C. 15 


\begin{tabular}{|c|c|}
\hline 拿 & 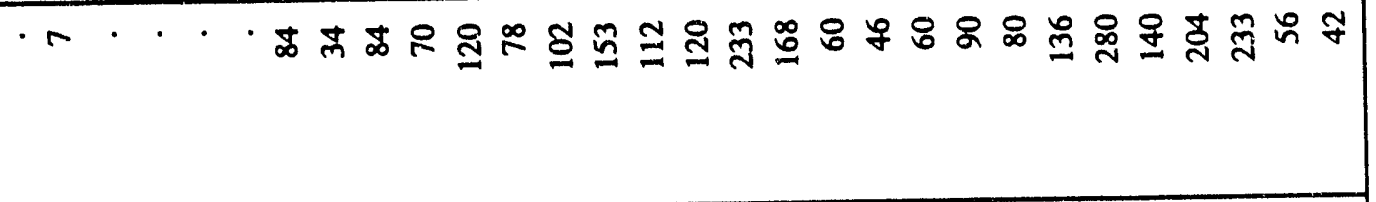 \\
\hline$\stackrel{8}{8}$ & 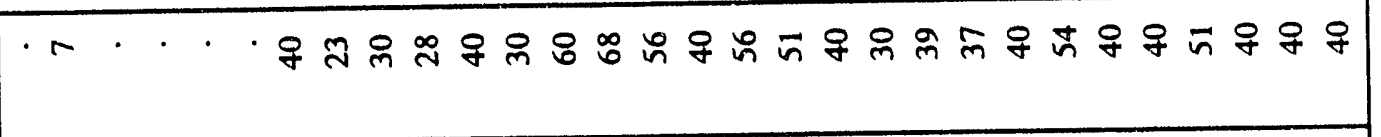 \\
\hline 遭 & 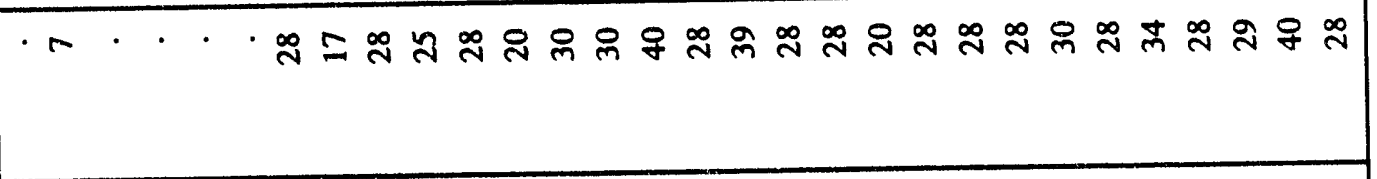 \\
\hline$\ddot{y}$ & 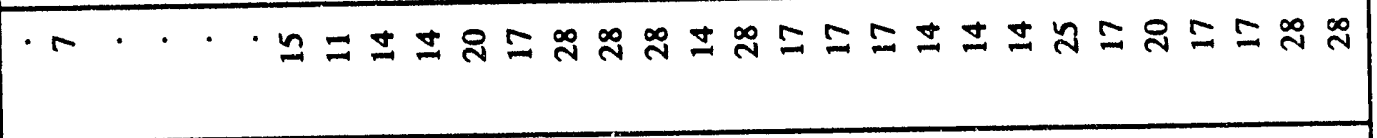 \\
\hline 要 & 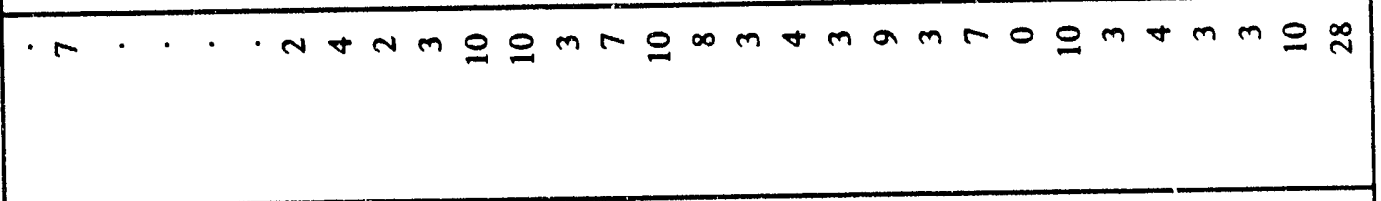 \\
\hline 窘 & 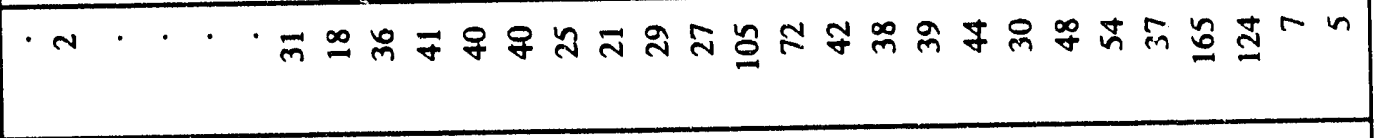 \\
\hline 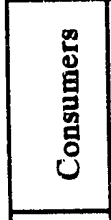 & 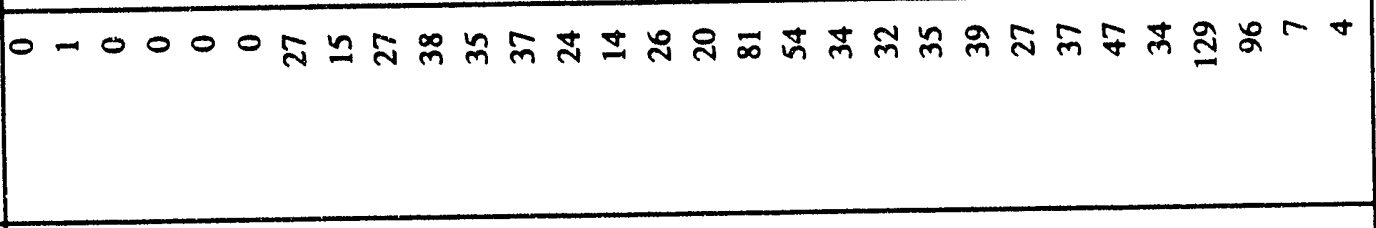 \\
\hline 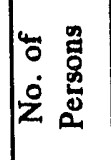 & 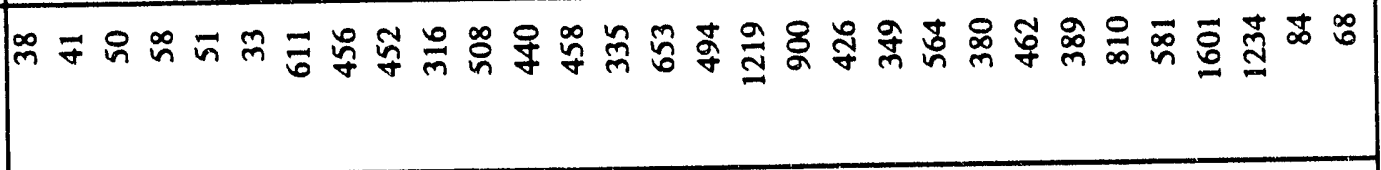 \\
\hline $\begin{array}{l}\text { 总 } \\
\text { 悬 } \\
\text { 点 }\end{array}$ & 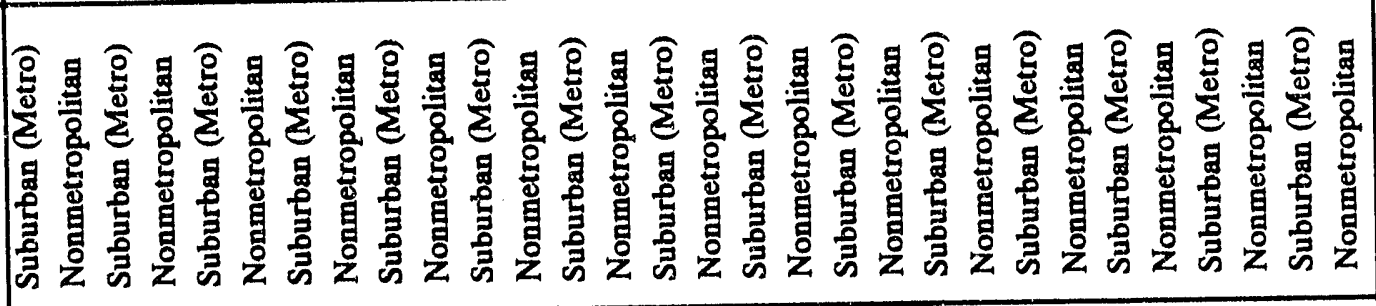 \\
\hline & 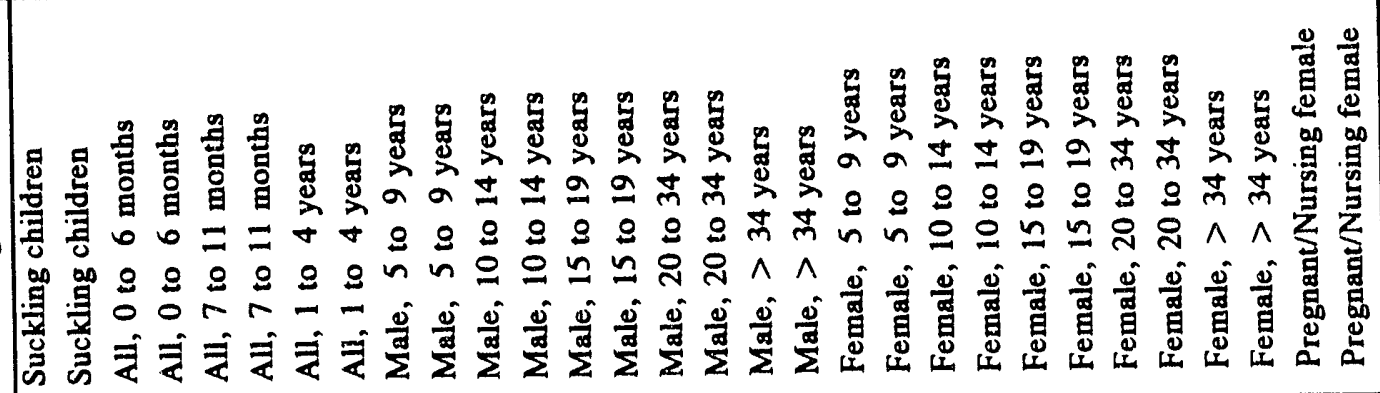 \\
\hline
\end{tabular}

C. 16 


\begin{tabular}{|c|c|}
\hline 量 & 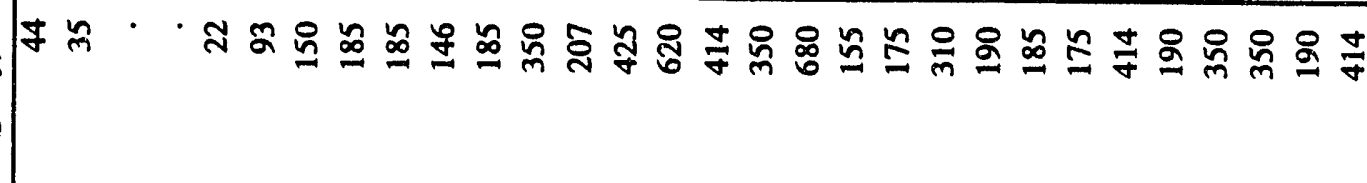 \\
\hline$\stackrel{80}{x}$ & 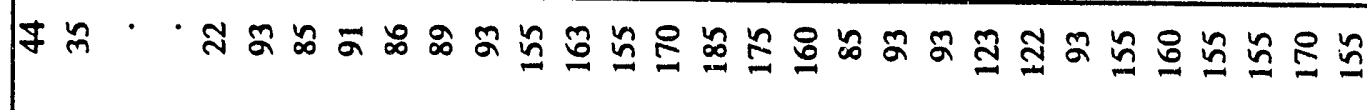 \\
\hline 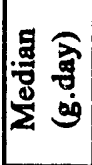 & 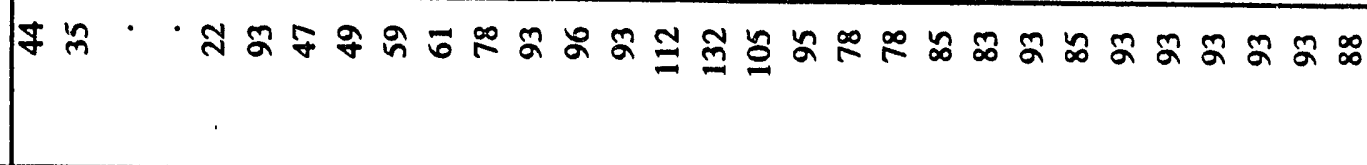 \\
\hline$\stackrel{y}{8}$ & F的・ \\
\hline$\frac{1}{3}$ & 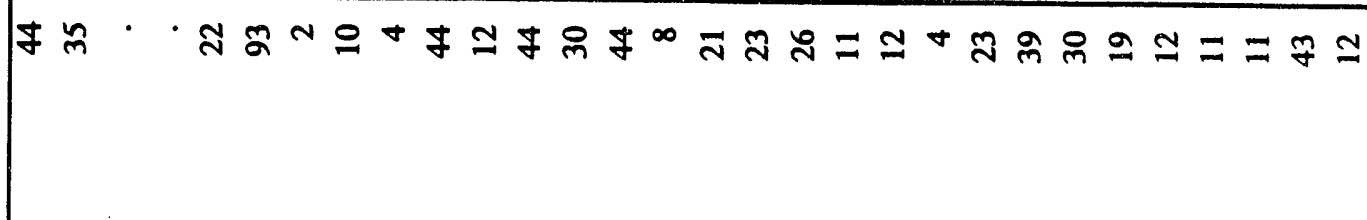 \\
\hline 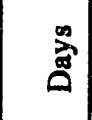 & 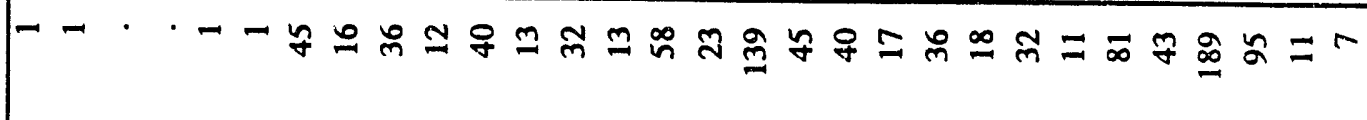 \\
\hline 惫 & 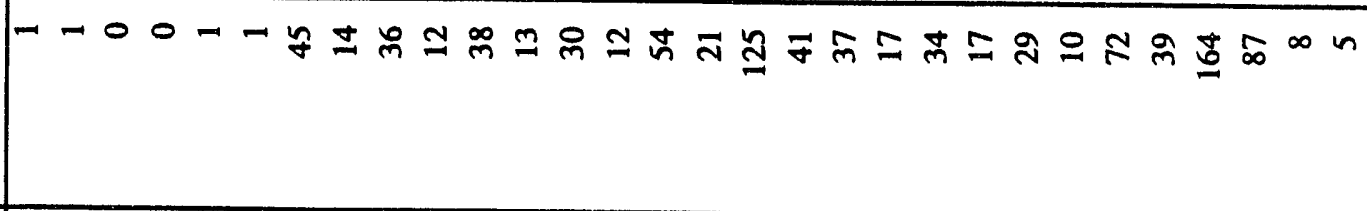 \\
\hline 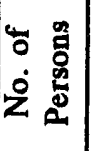 & 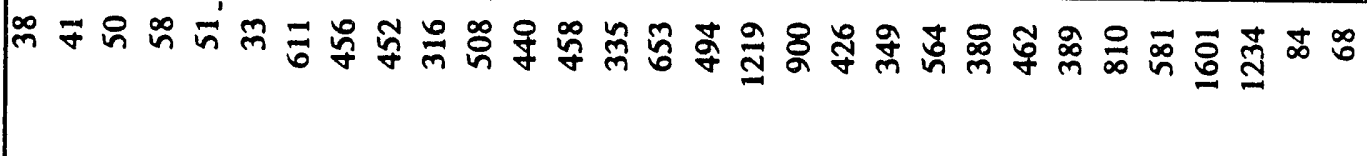 \\
\hline & 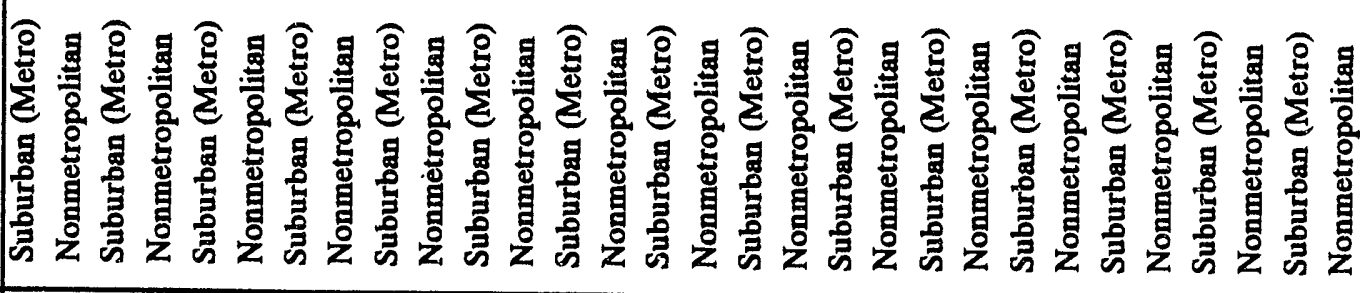 \\
\hline & 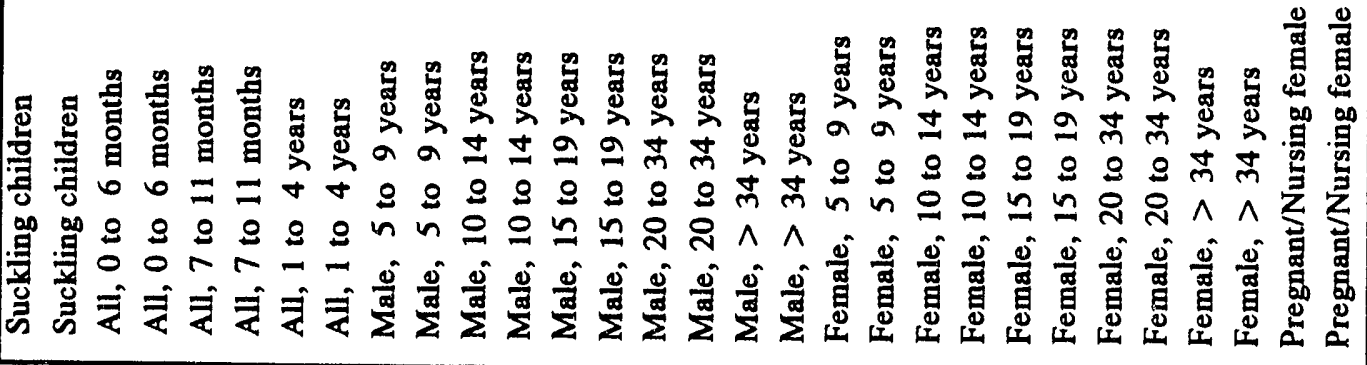 \\
\hline
\end{tabular}

C. 17 


\begin{tabular}{|c|c|}
\hline 要要 & 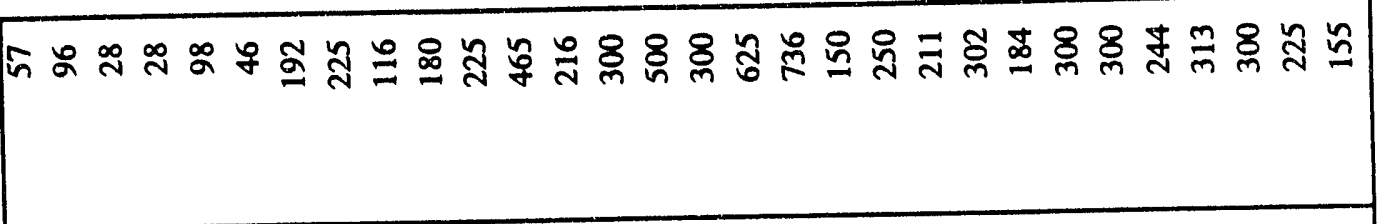 \\
\hline$\stackrel{8}{2}$ & 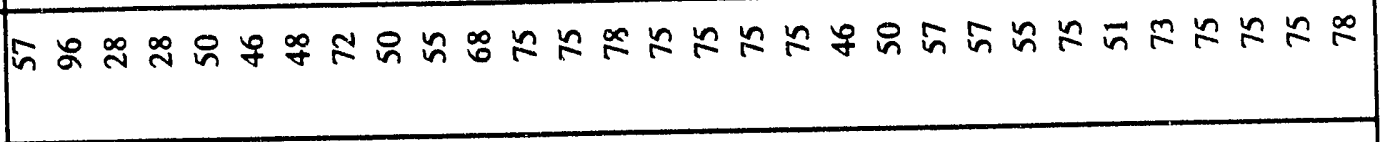 \\
\hline 要拿 & 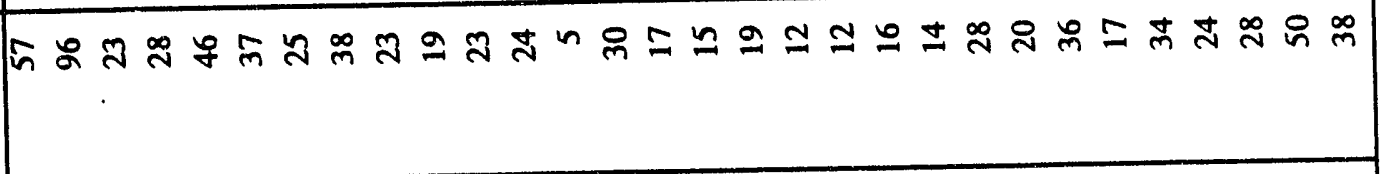 \\
\hline 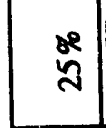 & 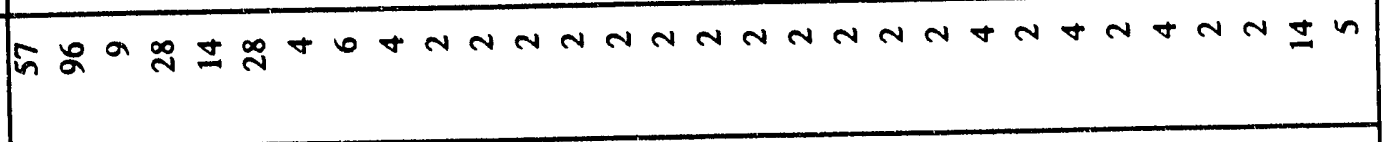 \\
\hline 㰾 & 的 \\
\hline$\stackrel{\infty}{\Delta}$ & 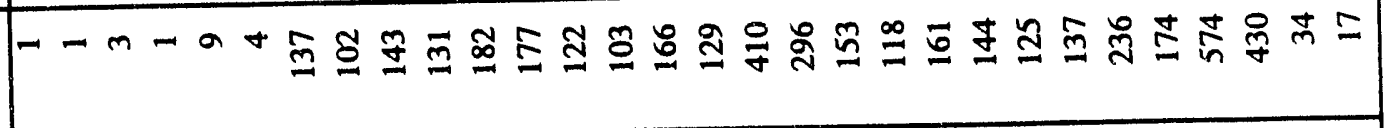 \\
\hline 恖 & - - - \\
\hline $\begin{array}{l}4 \\
0 \\
0 \\
\dot{2}\end{array}$ & 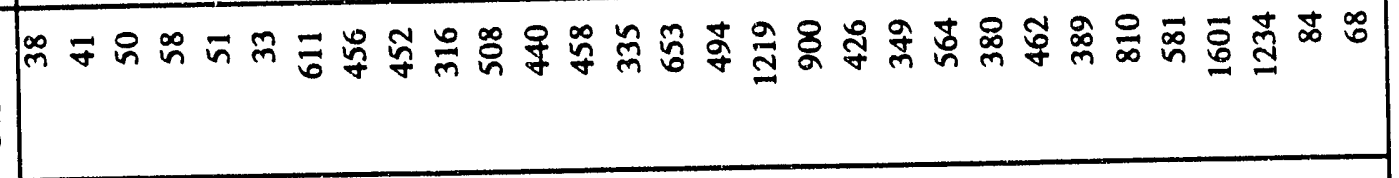 \\
\hline & 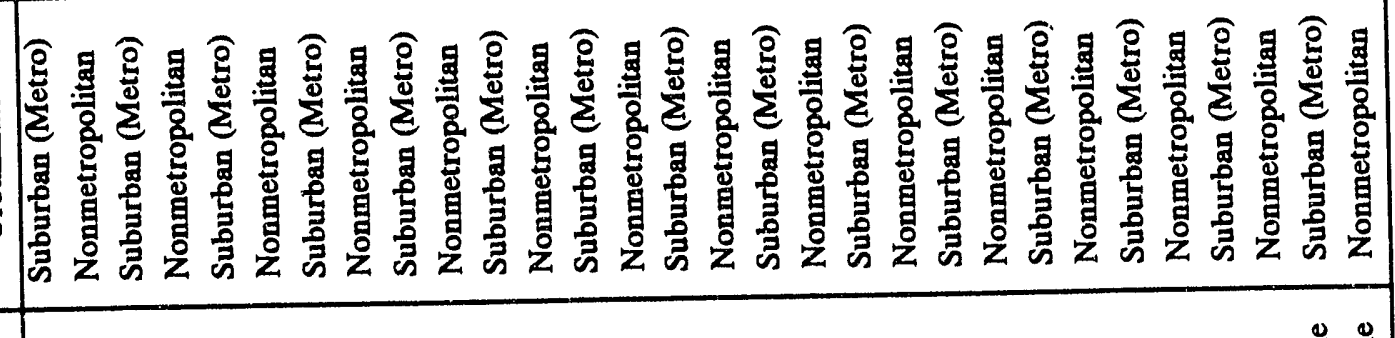 \\
\hline & 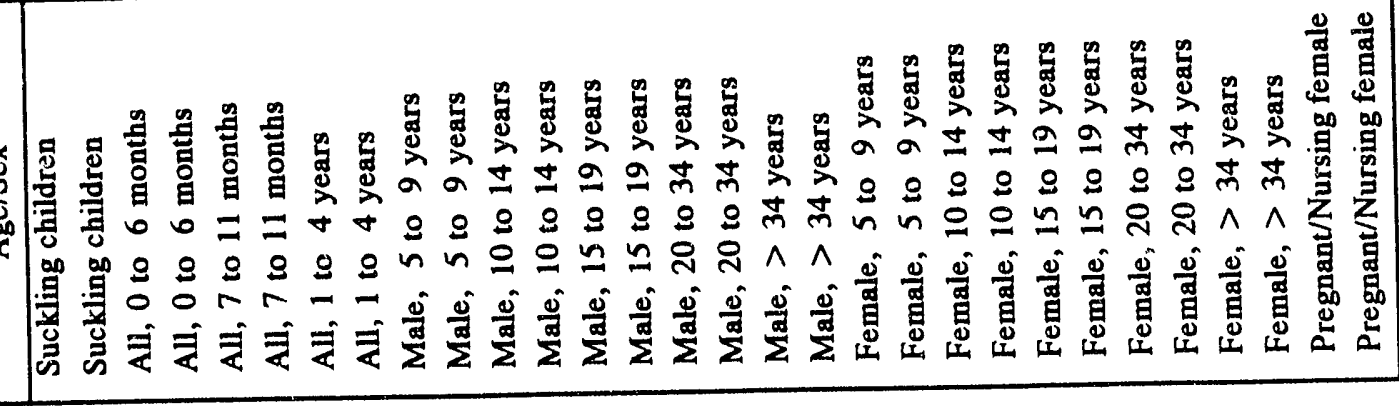 \\
\hline
\end{tabular}

C. 18 


\begin{tabular}{|c|c|}
\hline 量 & 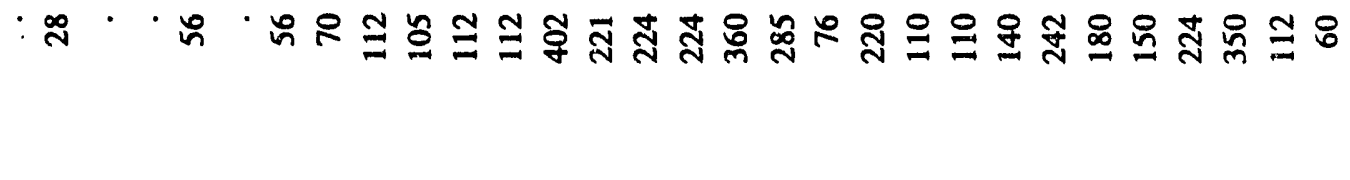 \\
\hline$\stackrel{8}{*}$ & 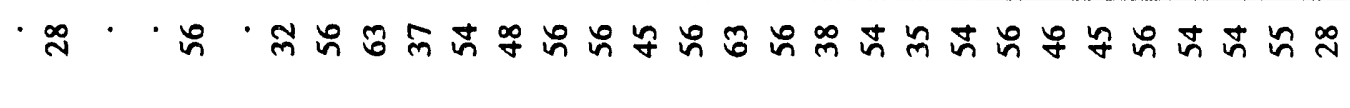 \\
\hline 通 & 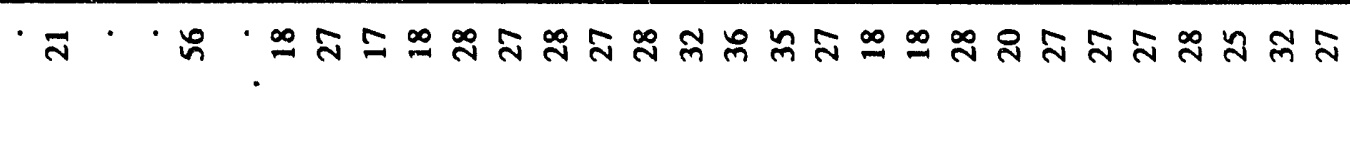 \\
\hline$\ddot{z}$ & 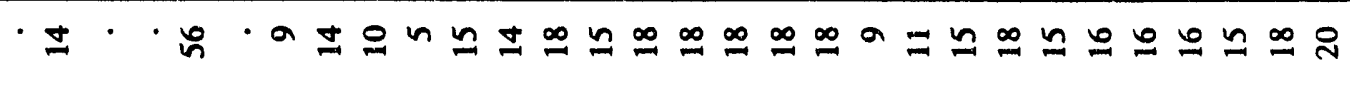 \\
\hline$\frac{1}{3}$ & $\Xi \cdot D_{n} \cdot 0-0 N-0 m-0-m-N N N-0-n \infty$ \\
\hline$\stackrel{n}{a}$ & 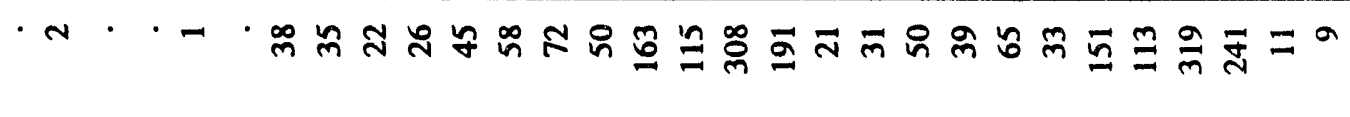 \\
\hline 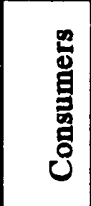 & 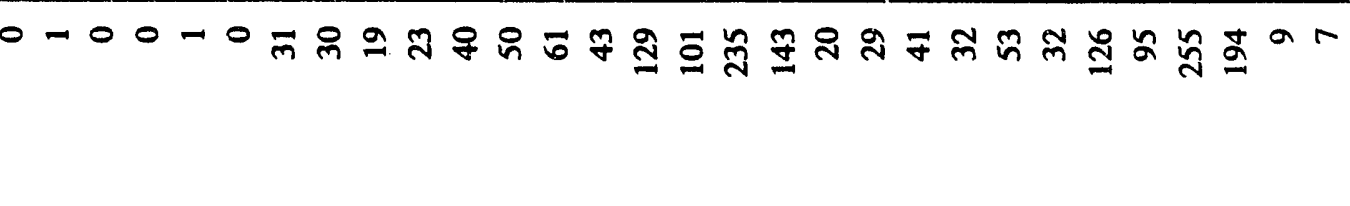 \\
\hline 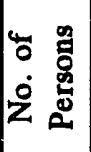 & 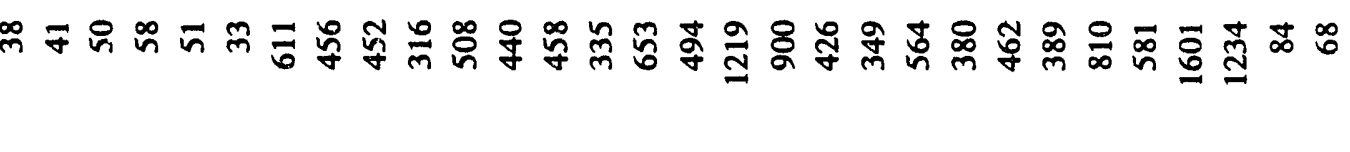 \\
\hline \multicolumn{2}{|r|}{ 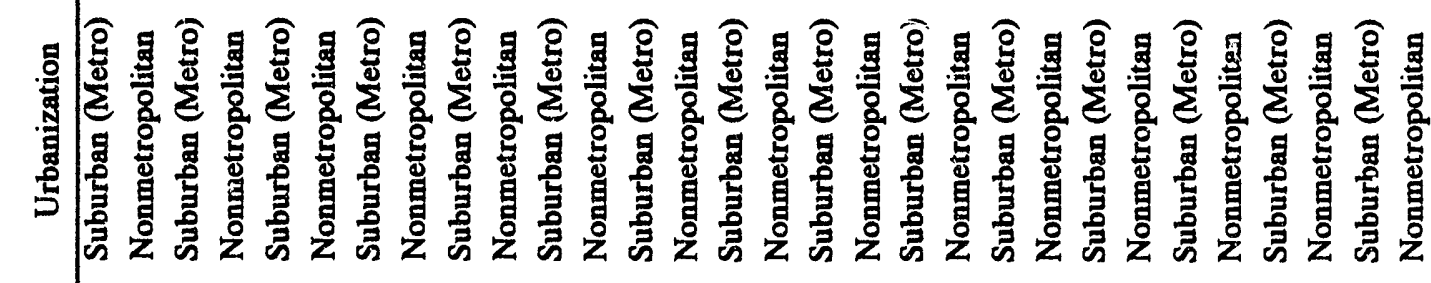 } \\
\hline 赵 & 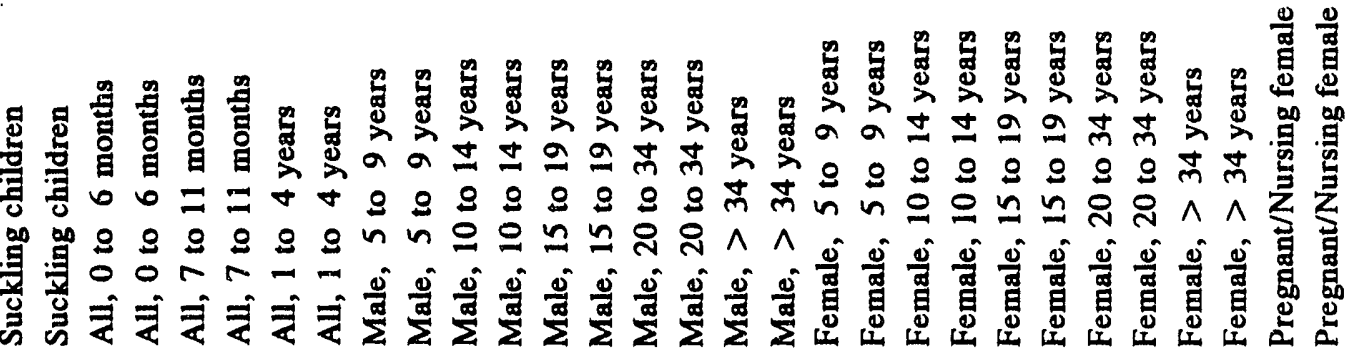 \\
\hline
\end{tabular}

C. 19 


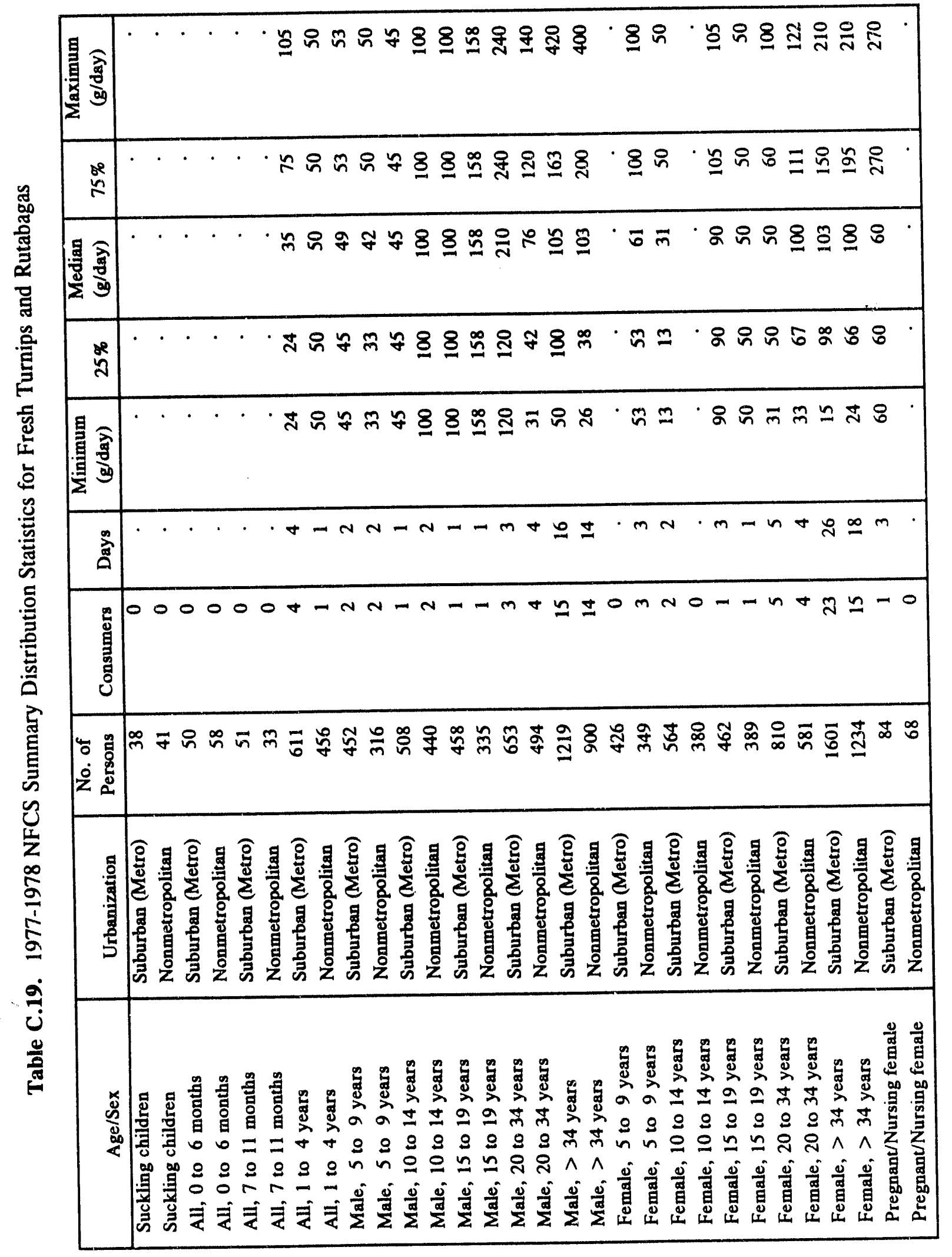

C. 20 


\begin{tabular}{|c|c|c|}
\hline 要 & $\triangleq \cong$ & 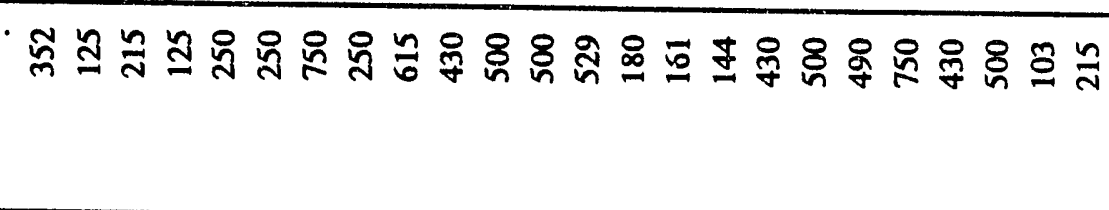 \\
\hline 年 & 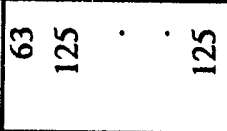 & 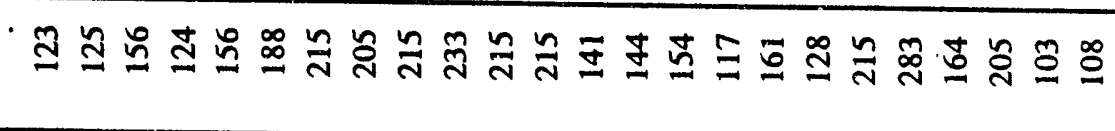 \\
\hline $\mid$ & 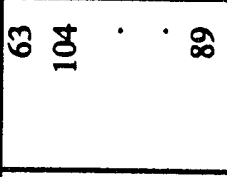 & 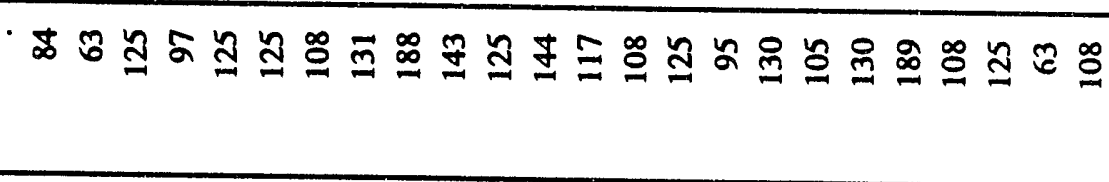 \\
\hline is & 8 & 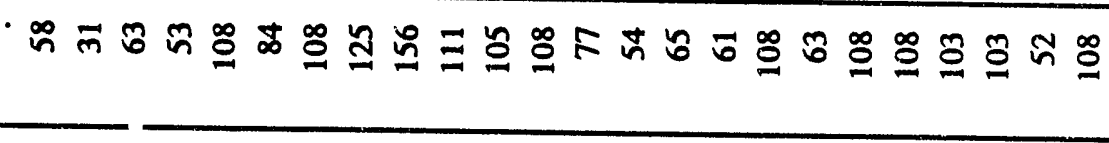 \\
\hline$\frac{1}{2}$ & 8 & 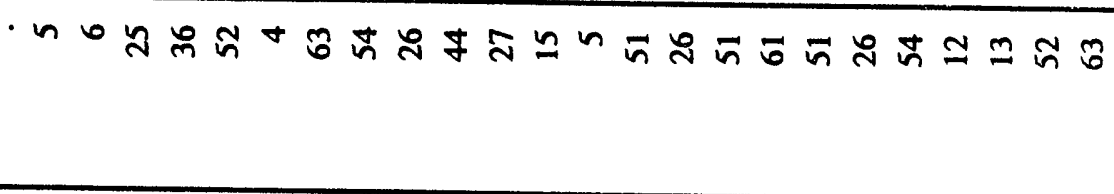 \\
\hline 商 & $-4 \cdot h$ & 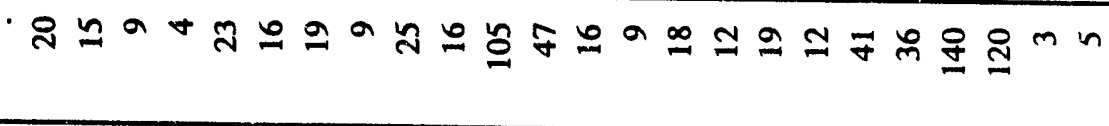 \\
\hline 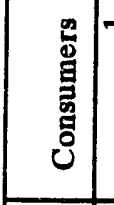 & r noon & 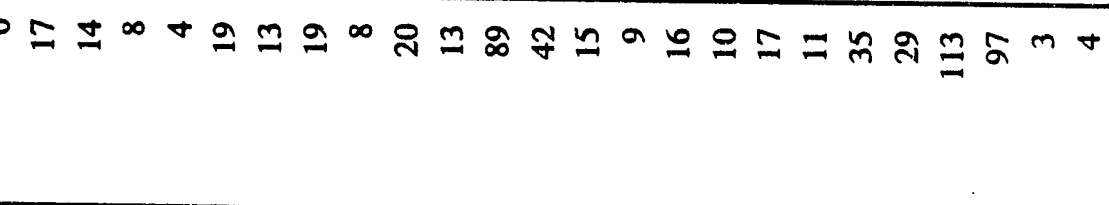 \\
\hline $\mid$ & m Fin & 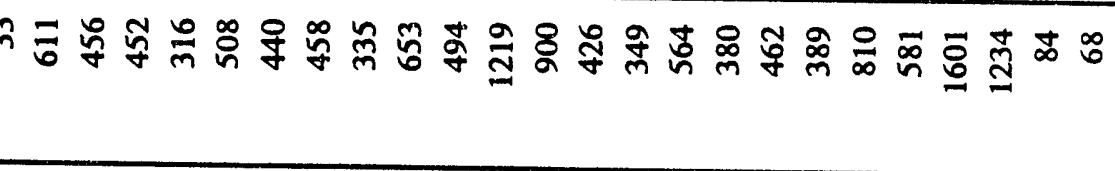 \\
\hline & 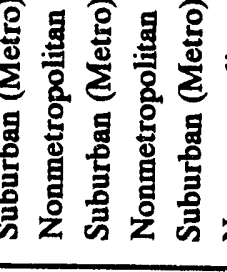 & 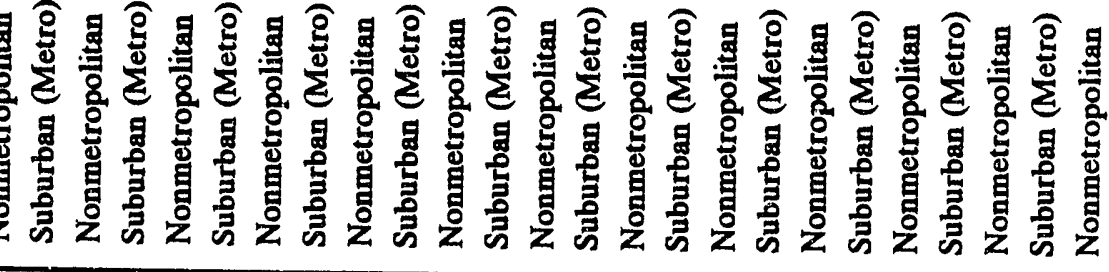 \\
\hline \multicolumn{3}{|c|}{ 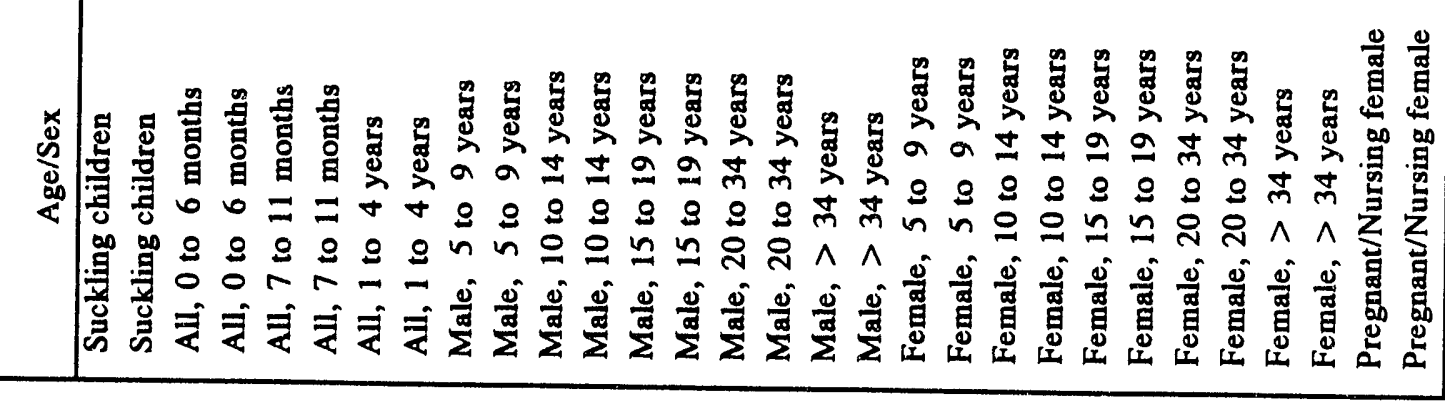 } \\
\hline
\end{tabular}




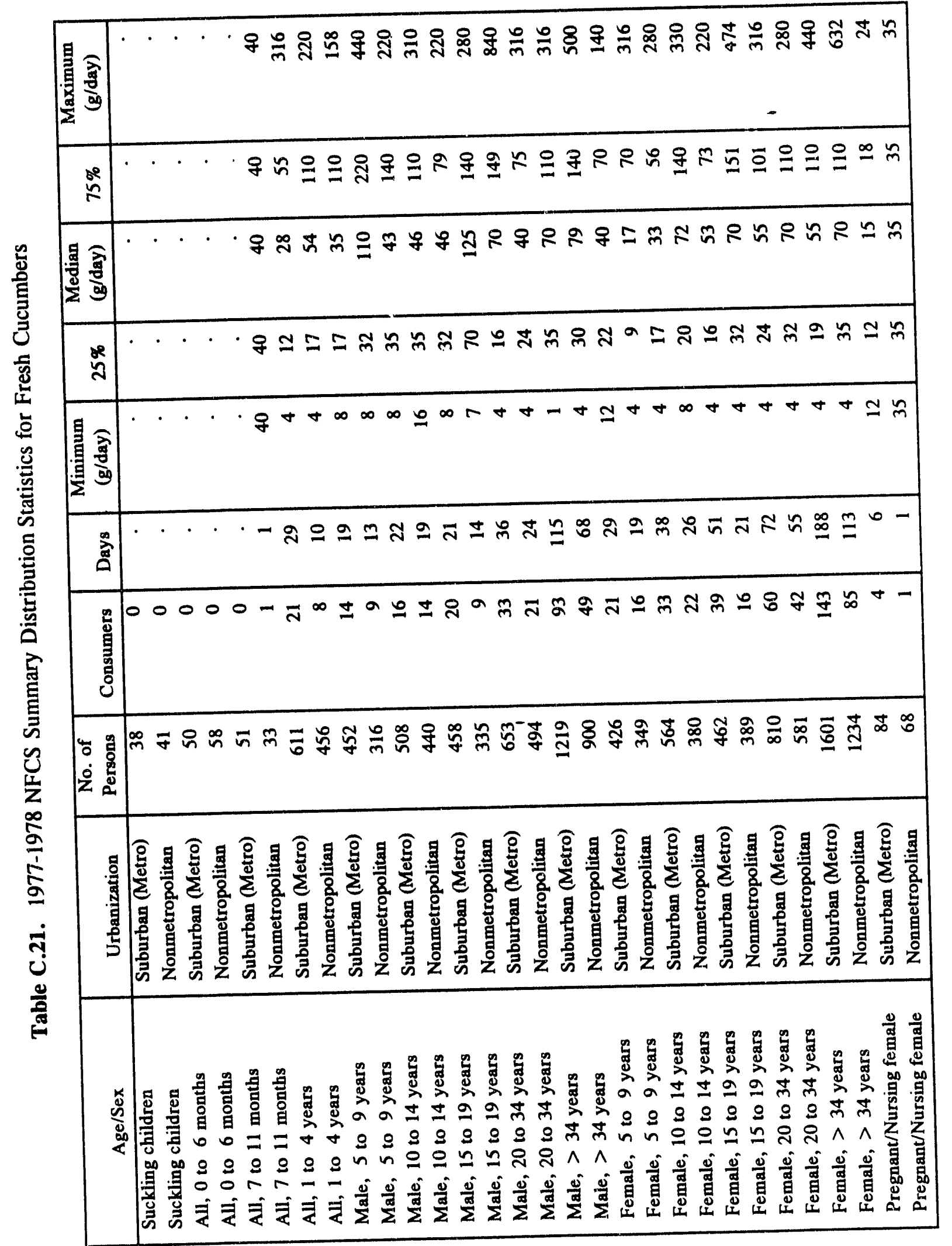

C. 22 


\begin{tabular}{|c|c|}
\hline$\frac{\mathrm{g}}{\mathrm{S}}$ & 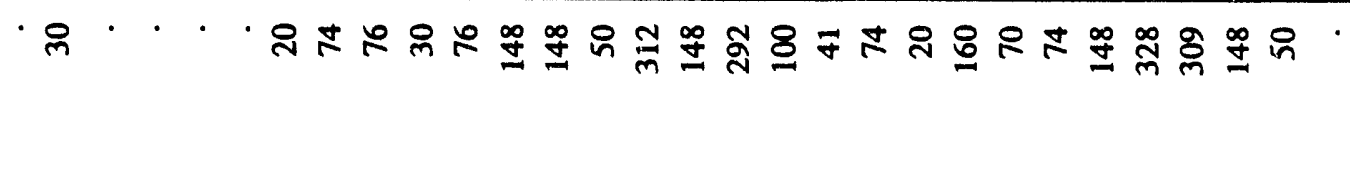 \\
\hline ڤ̊ & 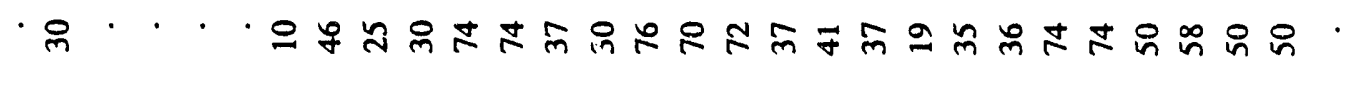 \\
\hline 蚍 & 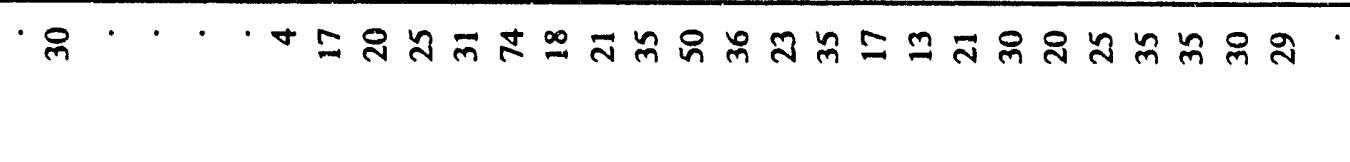 \\
\hline$\ddot{8}$ & 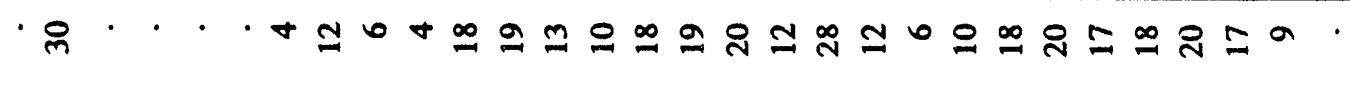 \\
\hline$\frac{1}{3}$ & $b \cdot \cdots \cdot n+\infty a \equiv 0 a n-\infty n+m N=\infty-\infty-0 a$ \\
\hline 离 & 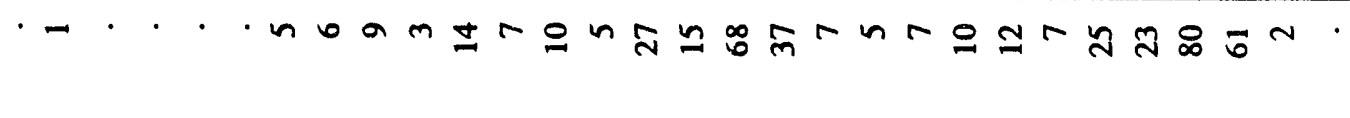 \\
\hline 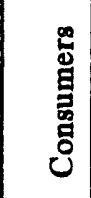 & 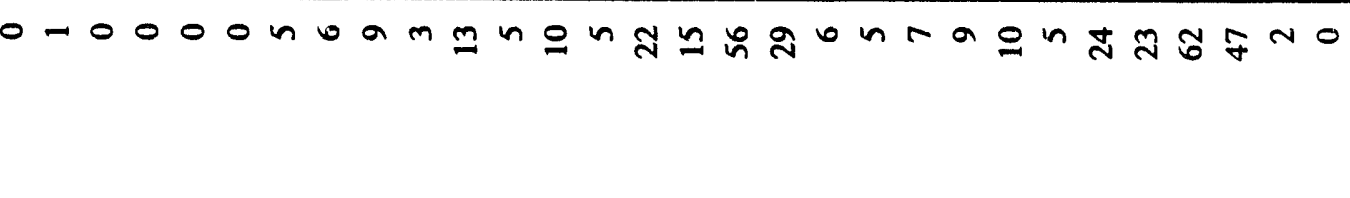 \\
\hline 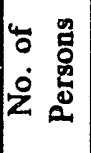 & 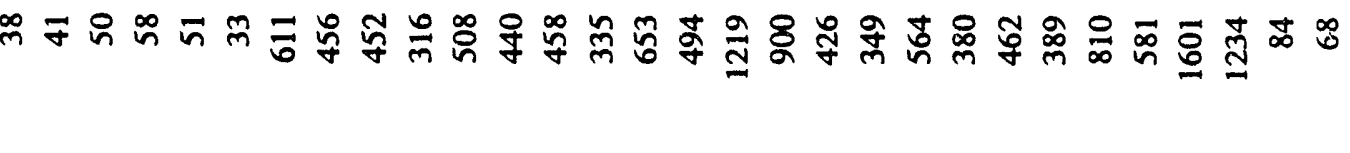 \\
\hline 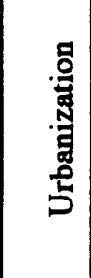 & 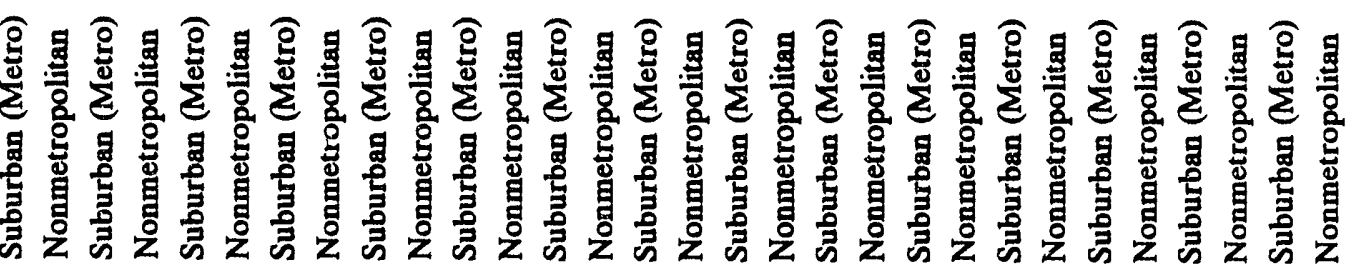 \\
\hline 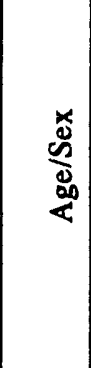 & 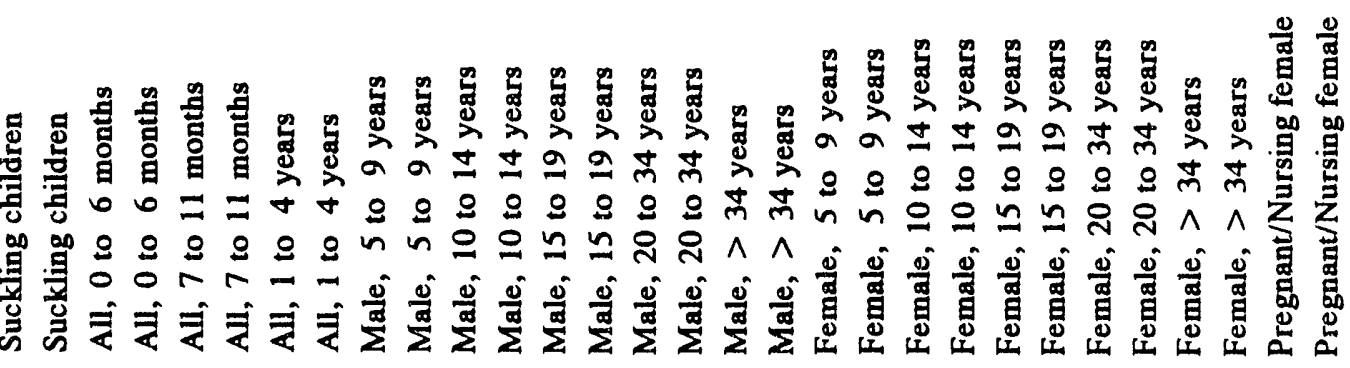 \\
\hline
\end{tabular}




\begin{tabular}{|c|c|}
\hline 罡蛋 & 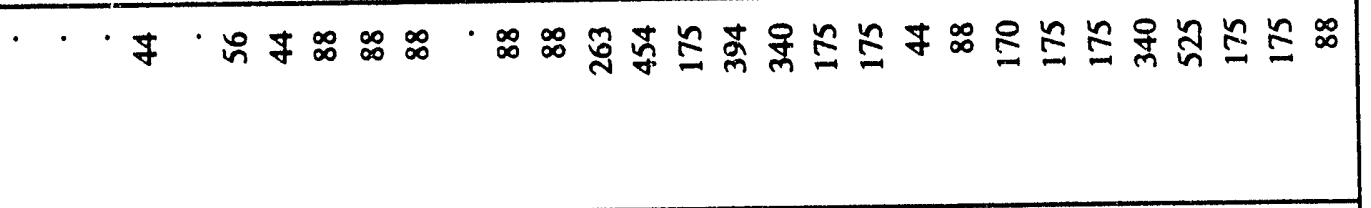 \\
\hline 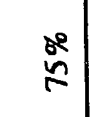 & 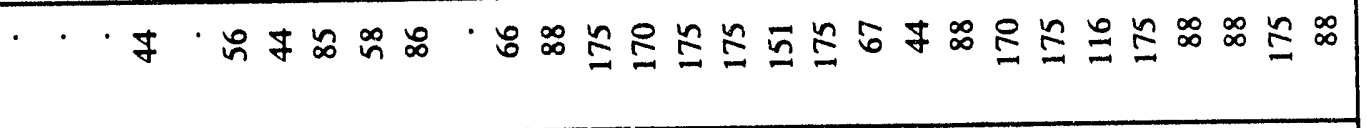 \\
\hline 蛋 & $\cdot \cdot \cdot \forall \cdot$ · \\
\hline$\ddot{s}$ & 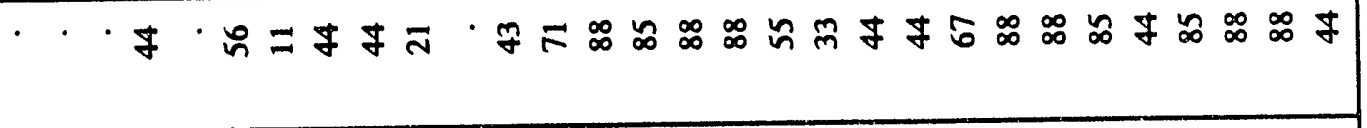 \\
\hline 悬票 & 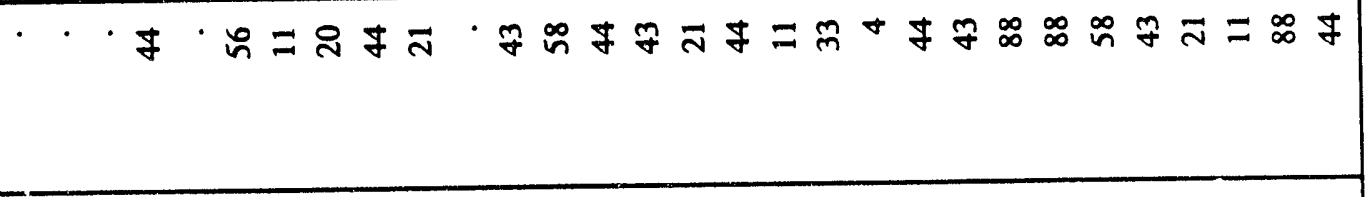 \\
\hline$\stackrel{\infty}{\hat{m}}$ & 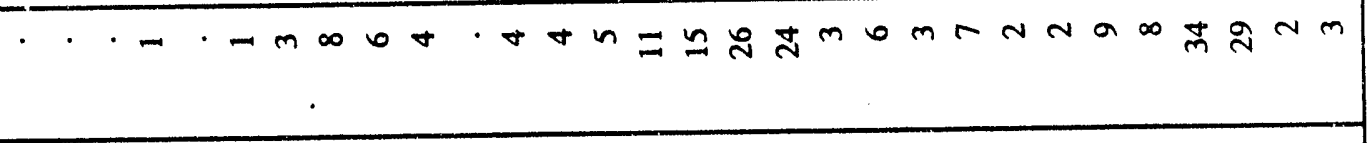 \\
\hline 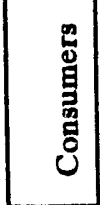 & $000-0 \cdots m \infty$ mot thang \\
\hline 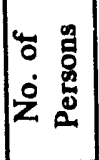 & 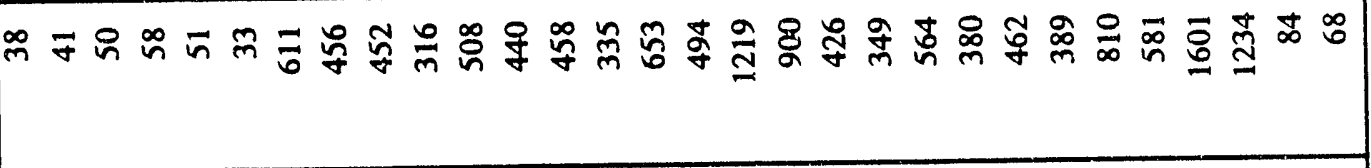 \\
\hline 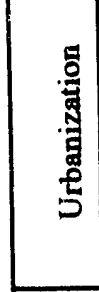 & 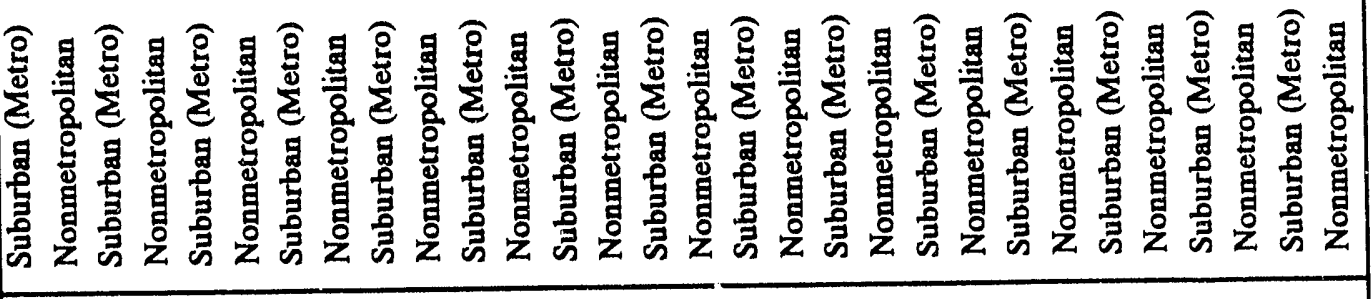 \\
\hline 离 & 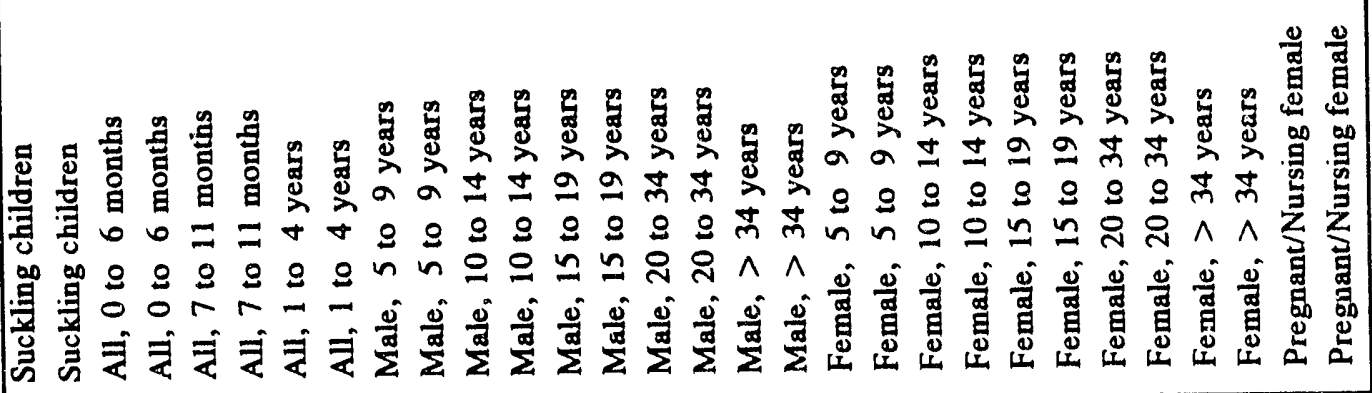 \\
\hline
\end{tabular}




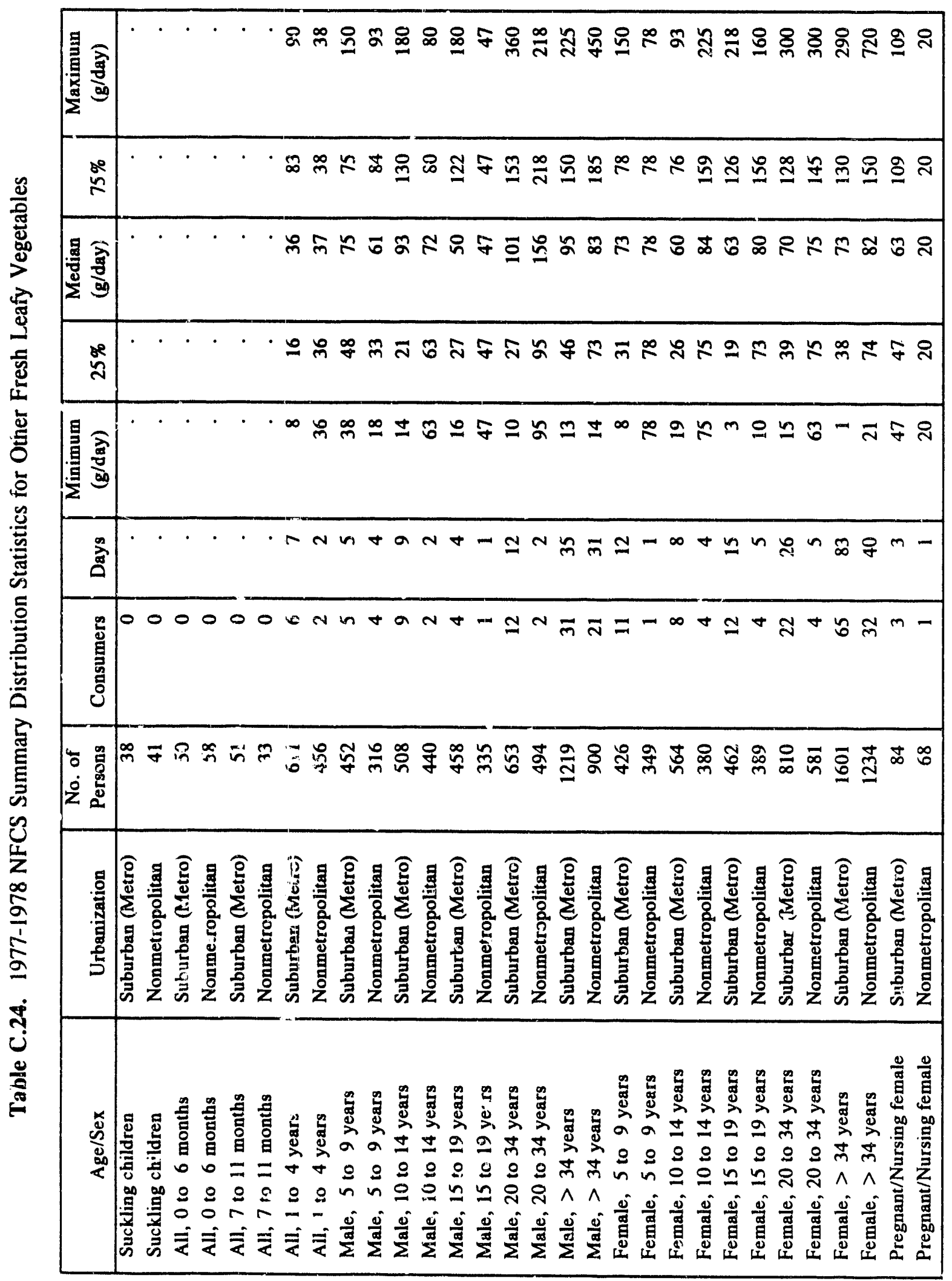

C. 25 


\begin{tabular}{|c|c|}
\hline 量离 & 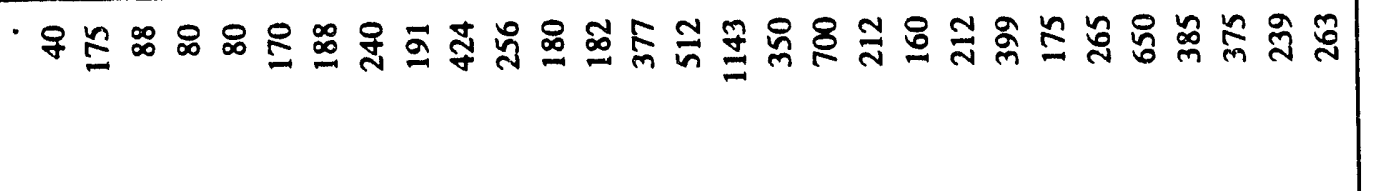 \\
\hline 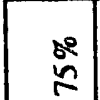 & 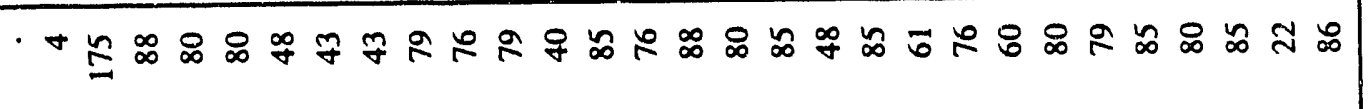 \\
\hline 递拿 & 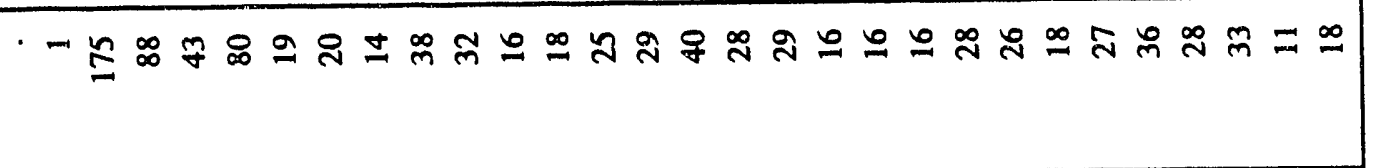 \\
\hline$\ddot{y}$ & 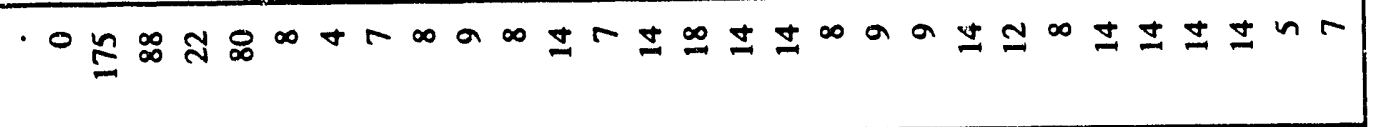 \\
\hline 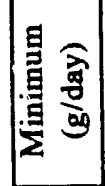 & $0 \leqq \infty$ ก \\
\hline 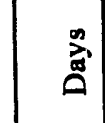 & 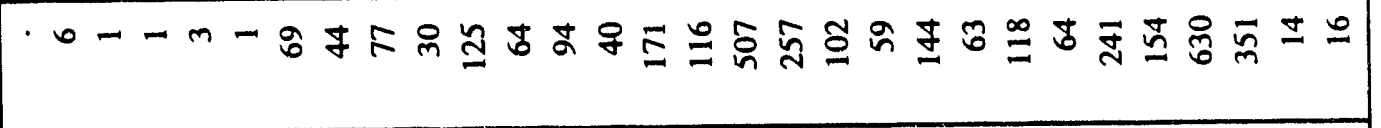 \\
\hline 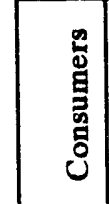 & 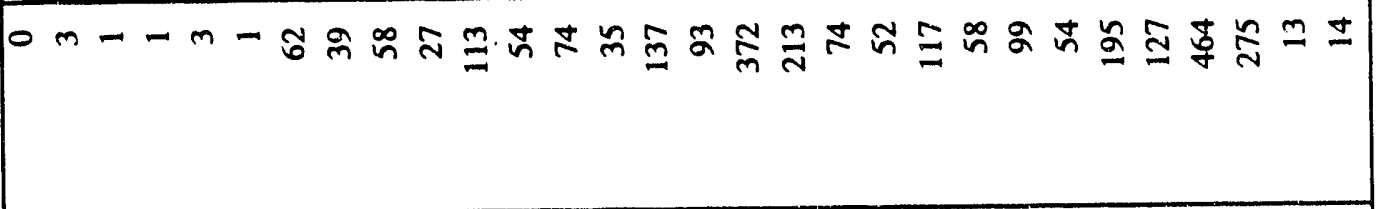 \\
\hline 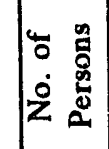 & 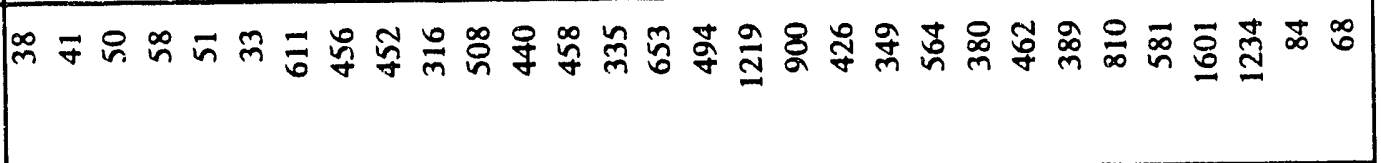 \\
\hline 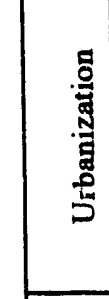 & 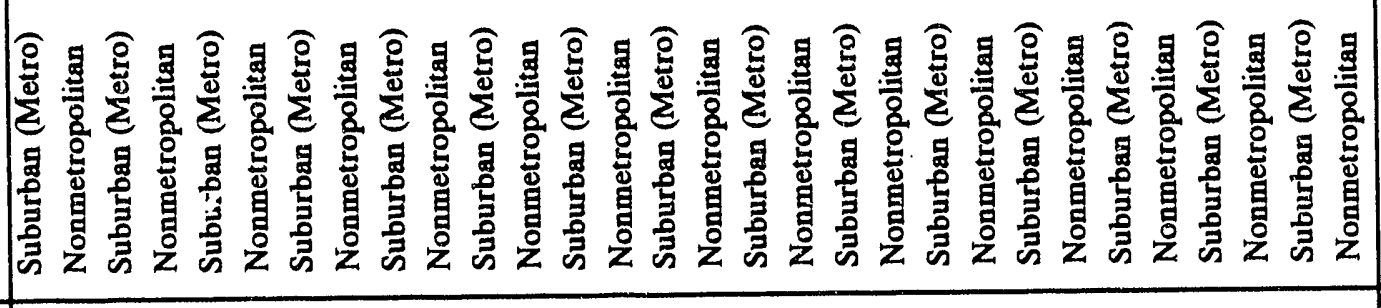 \\
\hline 象 & 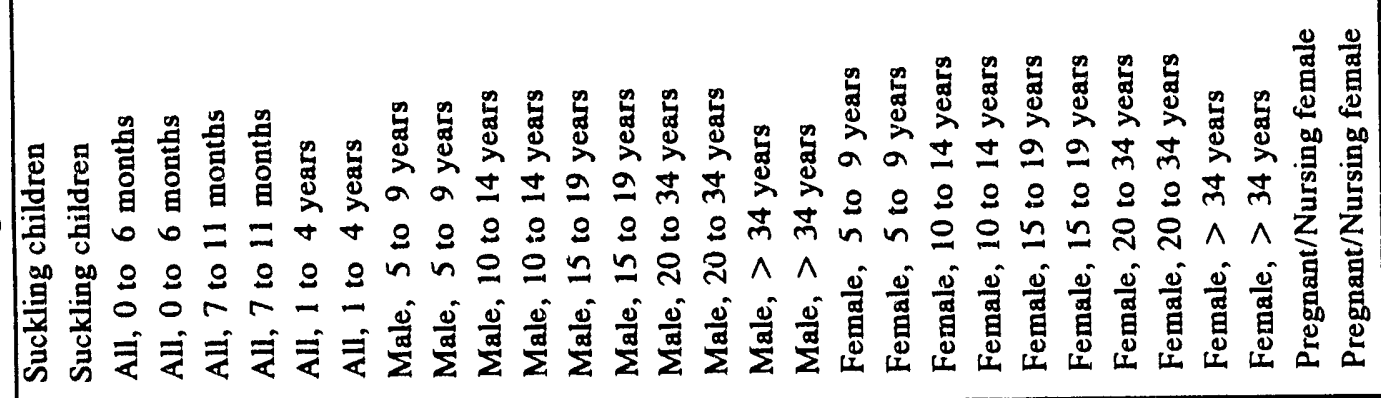 \\
\hline
\end{tabular}




\begin{tabular}{|c|c|}
\hline 量 & 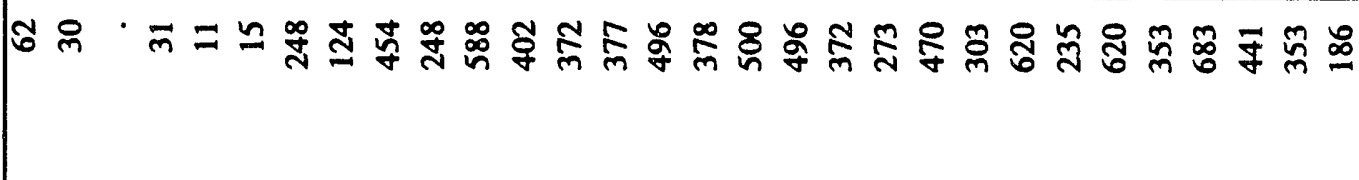 \\
\hline$\stackrel{20}{\Sigma}$ & 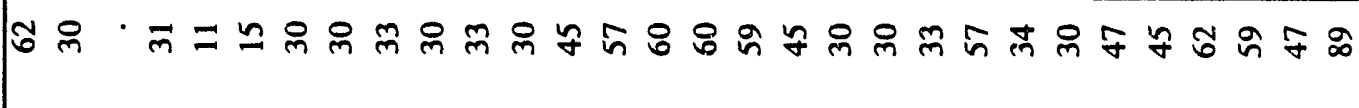 \\
\hline 通 & 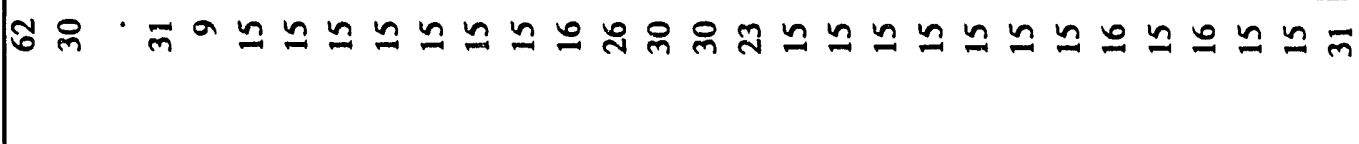 \\
\hline$y_{3}^{2}$ & 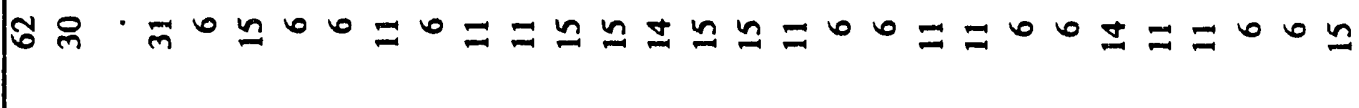 \\
\hline 量弯 & So \\
\hline 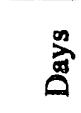 & 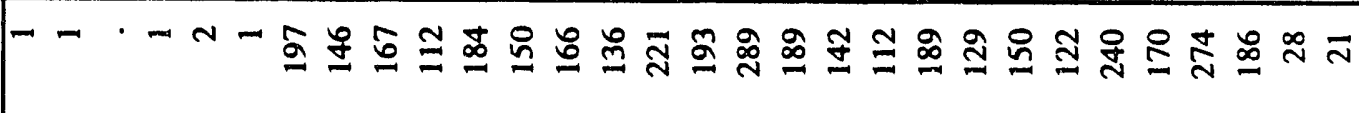 \\
\hline 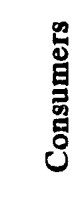 & 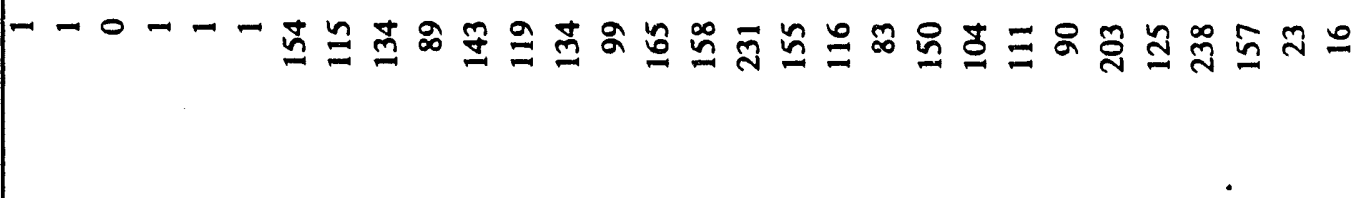 \\
\hline 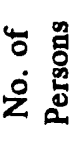 & 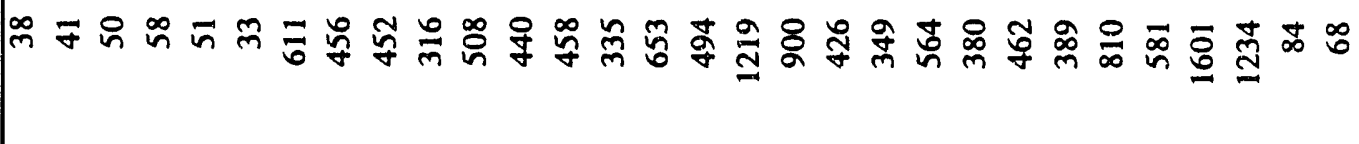 \\
\hline 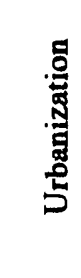 & 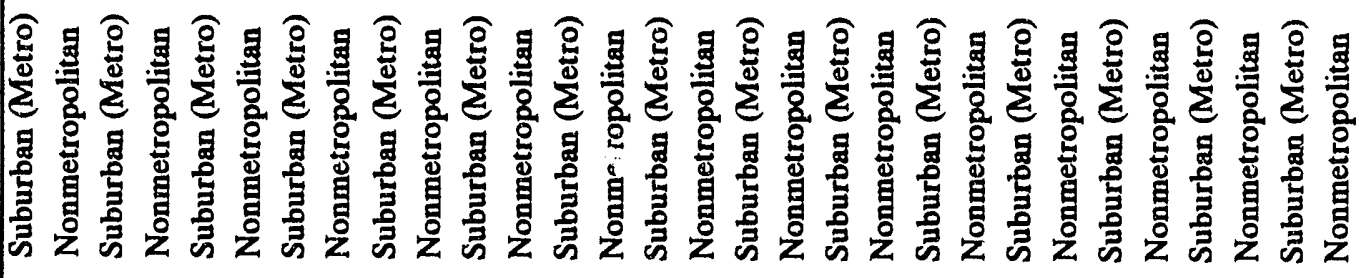 \\
\hline & 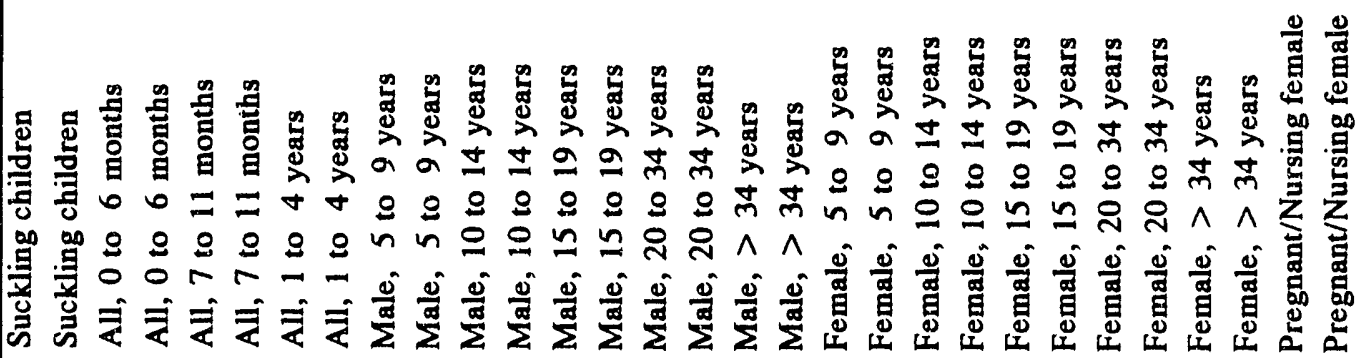 \\
\hline
\end{tabular}




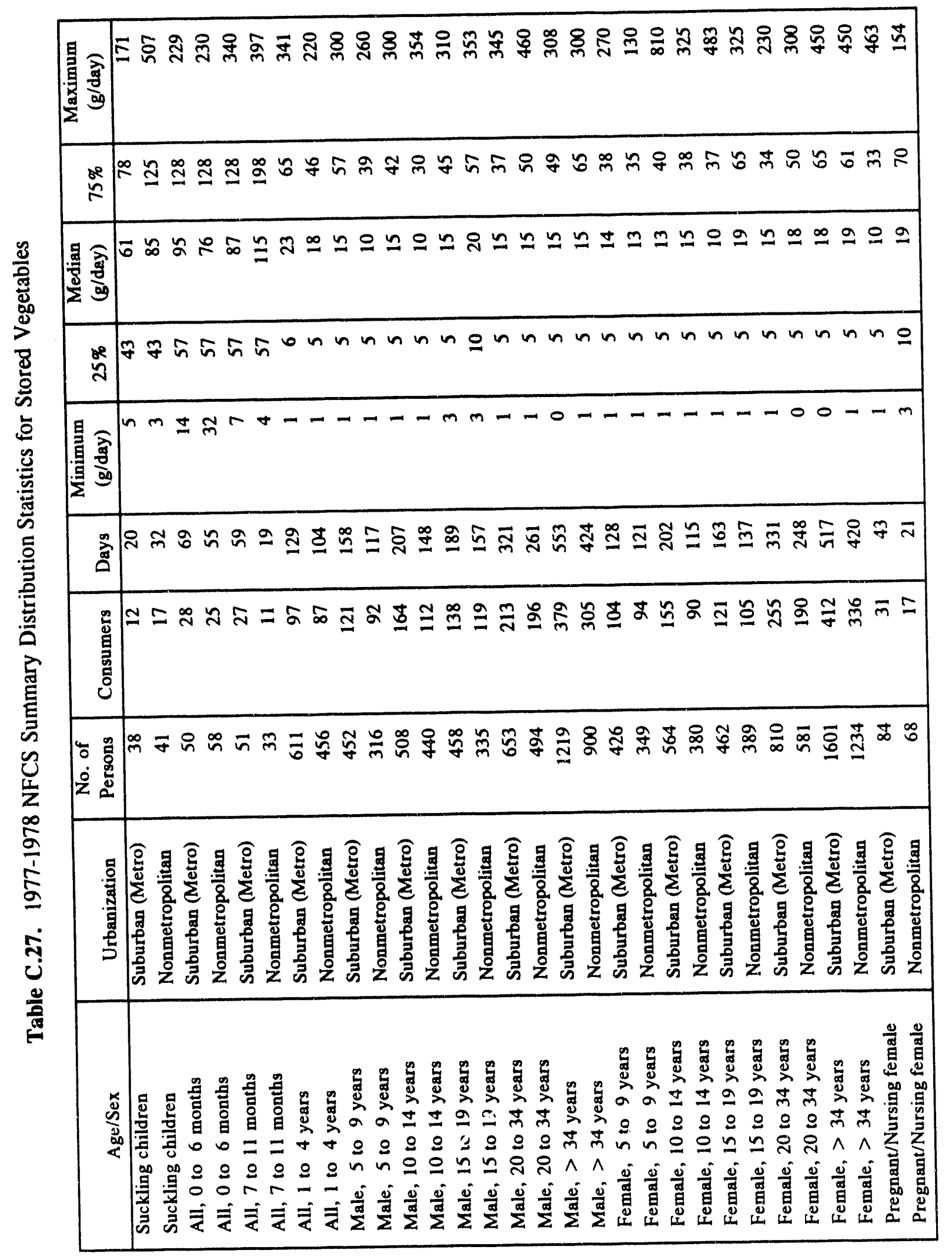

C. 28 


\begin{tabular}{|c|c|}
\hline 量拿 & 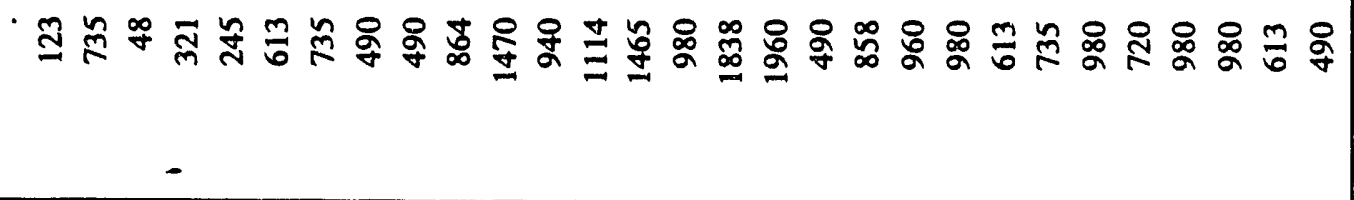 \\
\hline ڤ̊ & 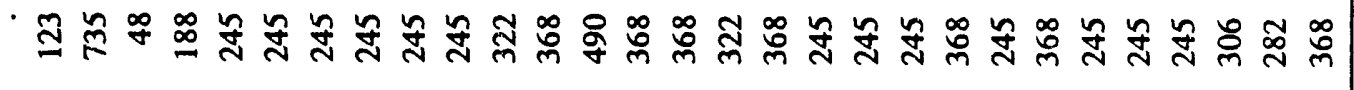 \\
\hline 这 & 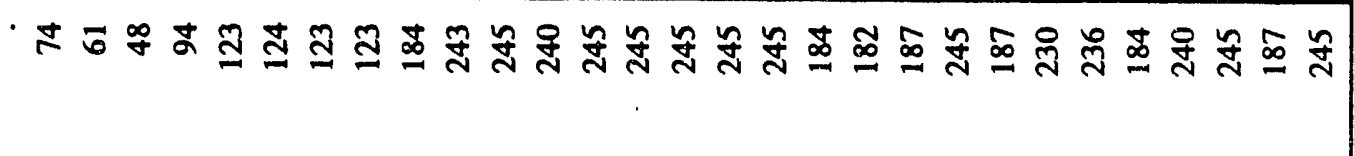 \\
\hline$\stackrel{8}{3}$ & 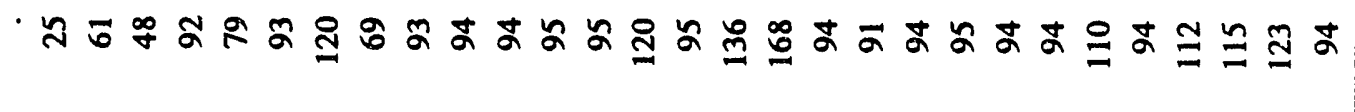 \\
\hline$\frac{1}{2}$ & 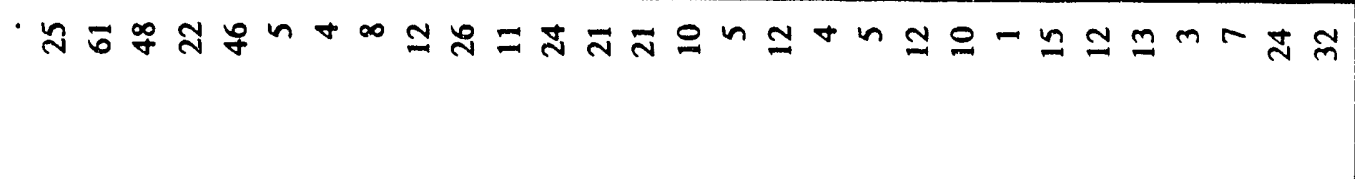 \\
\hline$\stackrel{\sim}{\hat{\Delta}}$ & 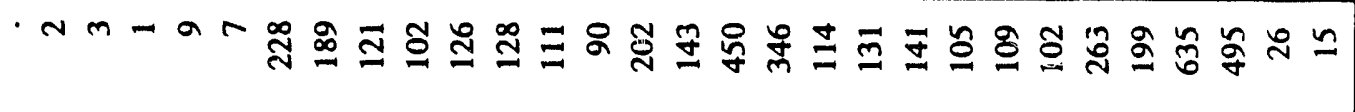 \\
\hline 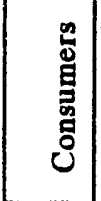 & 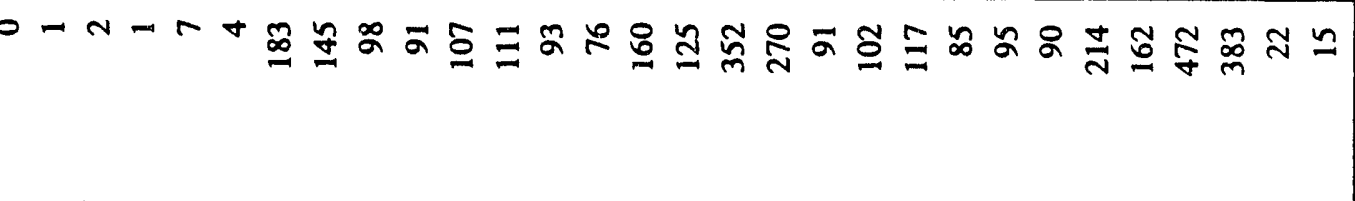 \\
\hline 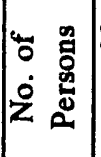 & 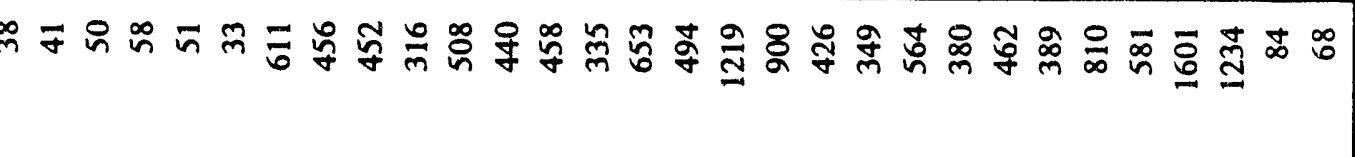 \\
\hline & 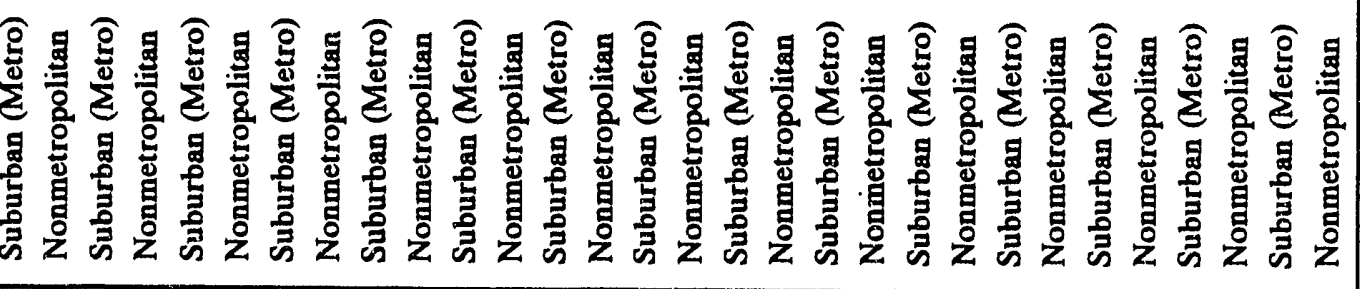 \\
\hline & 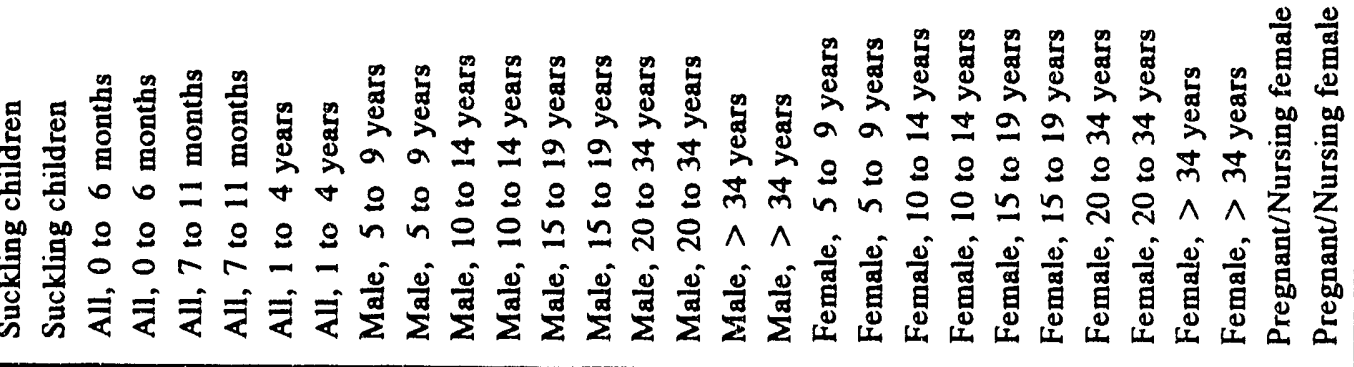 \\
\hline
\end{tabular}




\begin{tabular}{|c|c|}
\hline 量 & 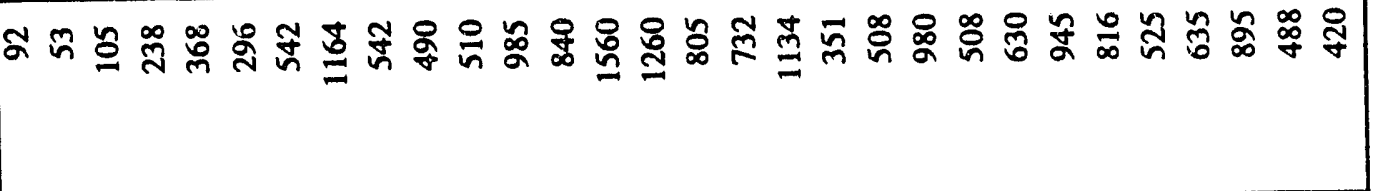 \\
\hline$\therefore$ & 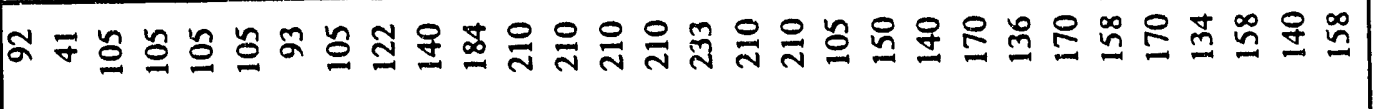 \\
\hline 管 & 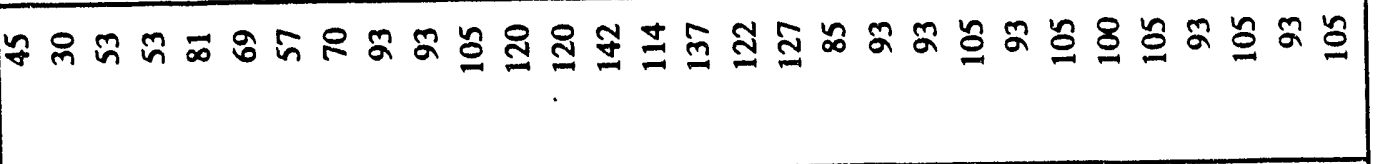 \\
\hline$x^{\circ}$ & 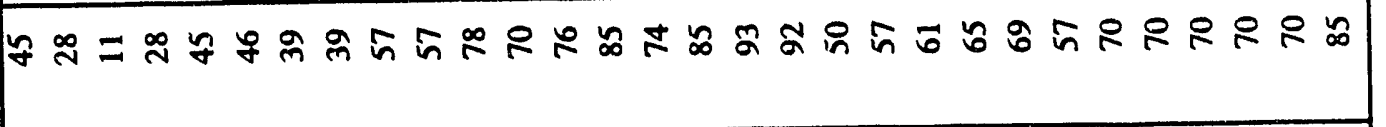 \\
\hline 量 & 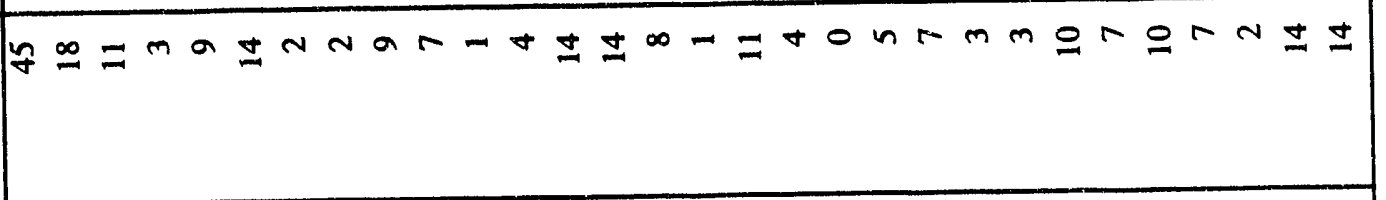 \\
\hline$\stackrel{\infty}{\hat{\Xi}}$ & 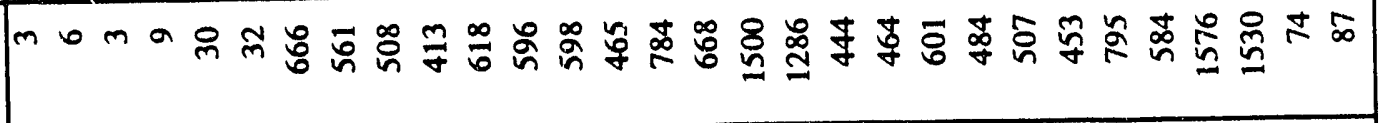 \\
\hline 总 & 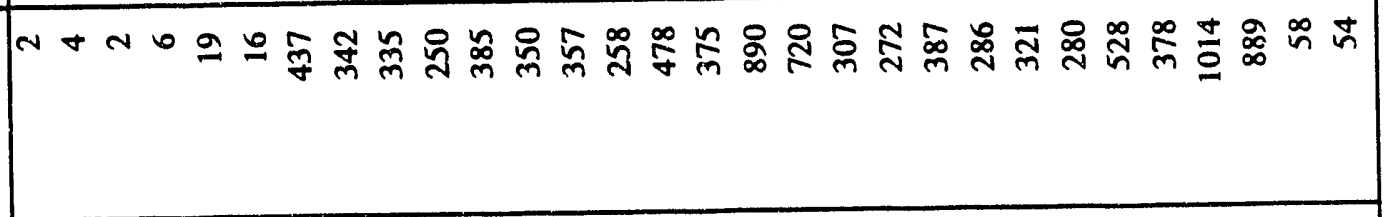 \\
\hline 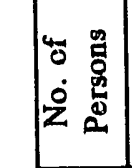 & 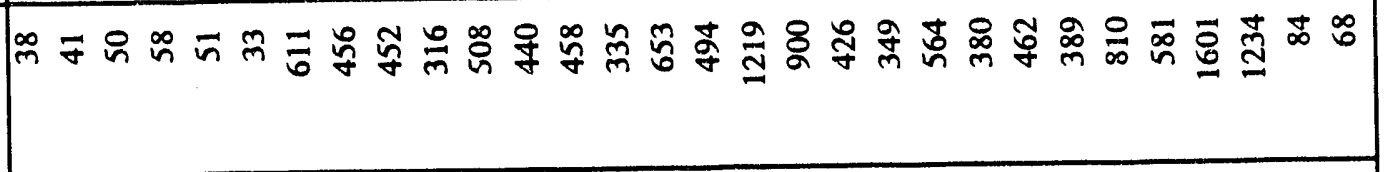 \\
\hline 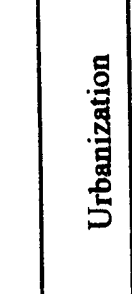 & 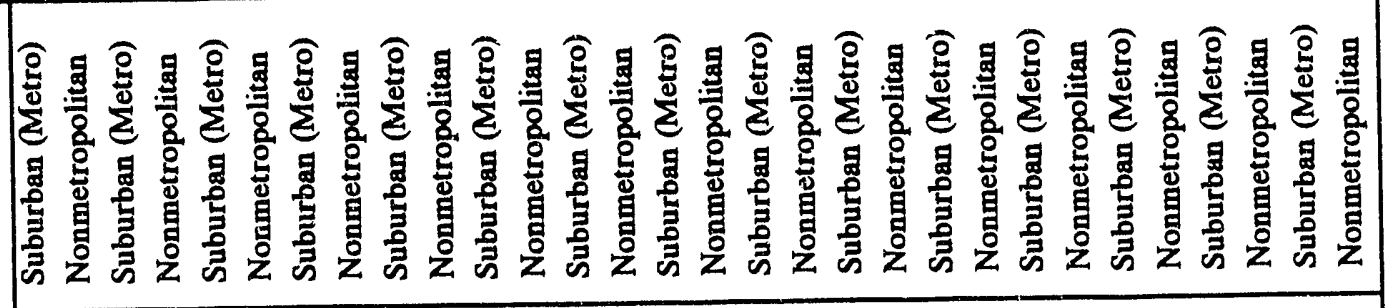 \\
\hline 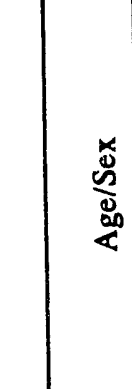 & 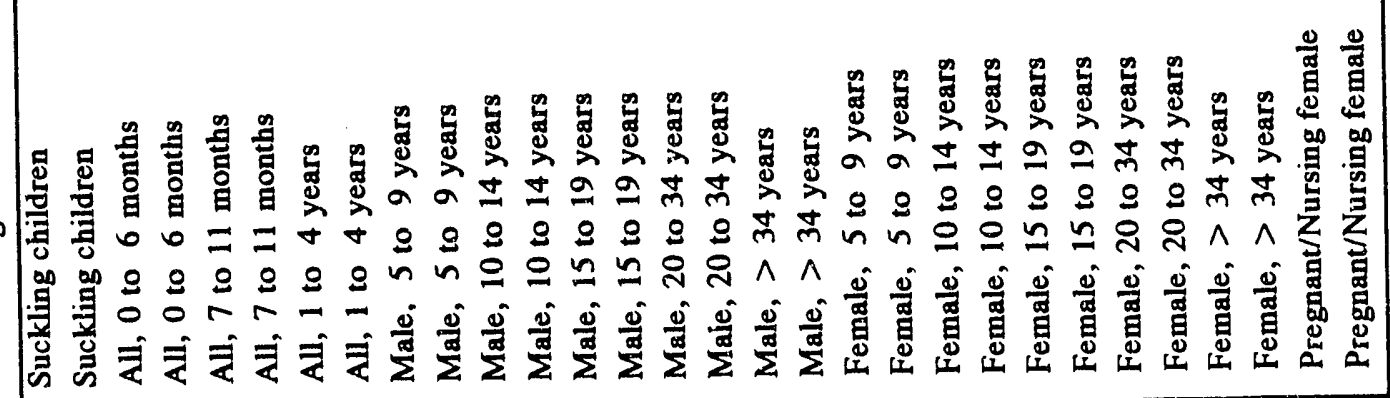 \\
\hline
\end{tabular}




\begin{tabular}{|c|c|}
\hline$\frac{1}{3}$ & 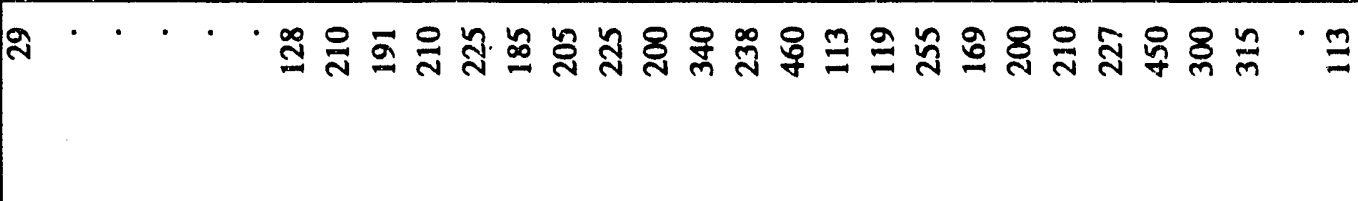 \\
\hline 象 & 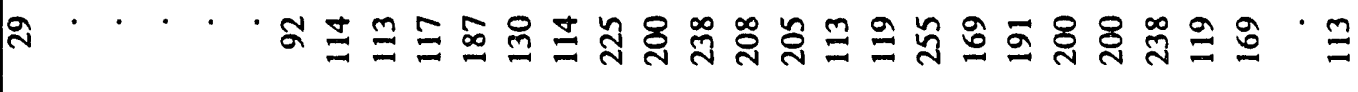 \\
\hline$\sum_{0}^{0}$ & 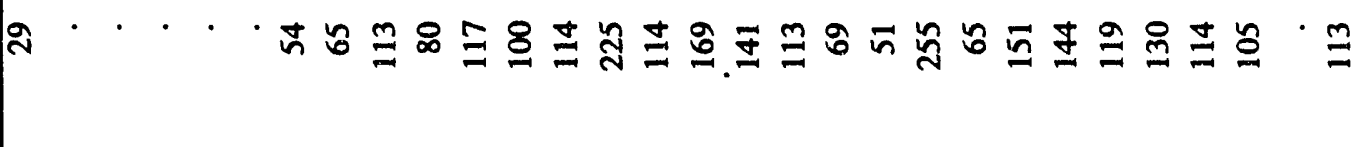 \\
\hline$\ddot{a}$ & 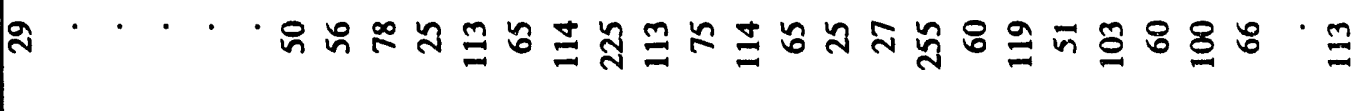 \\
\hline 量 & 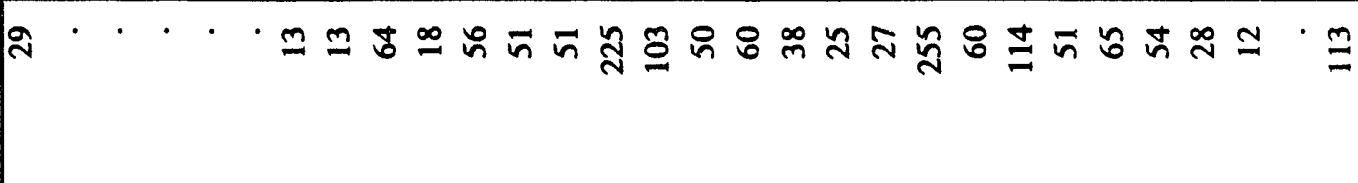 \\
\hline 离 & 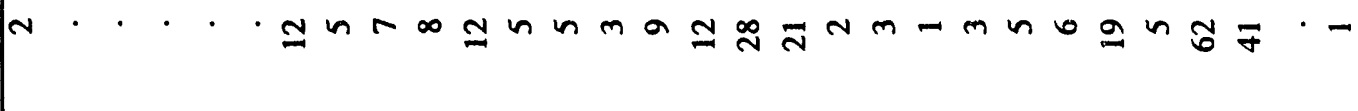 \\
\hline 惫 & 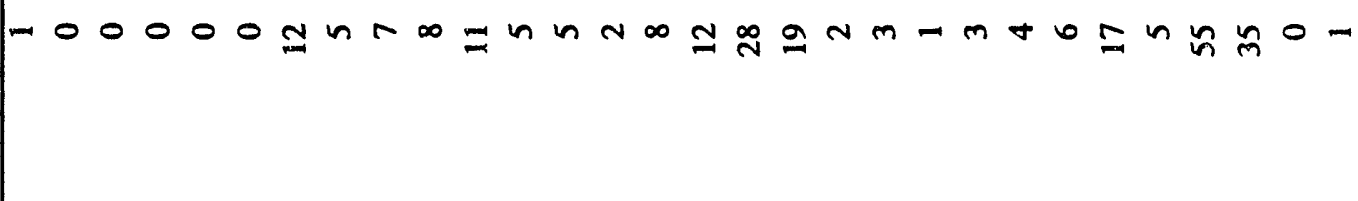 \\
\hline 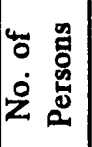 & 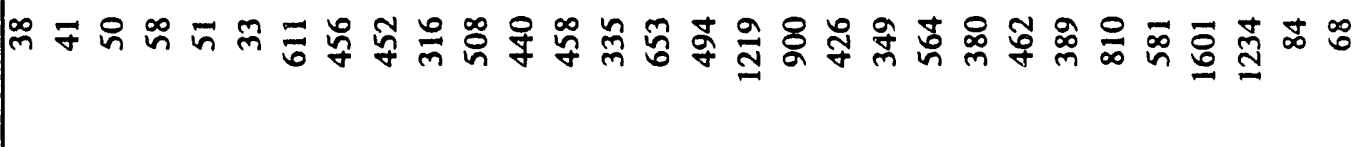 \\
\hline : & 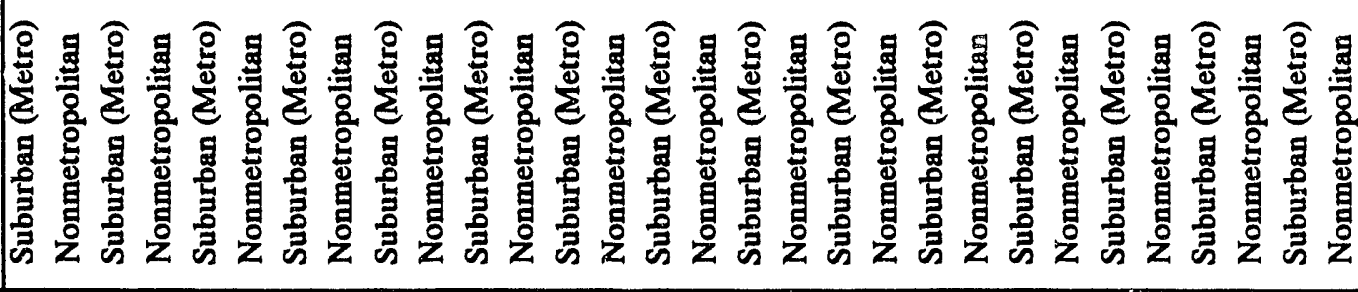 \\
\hline | & 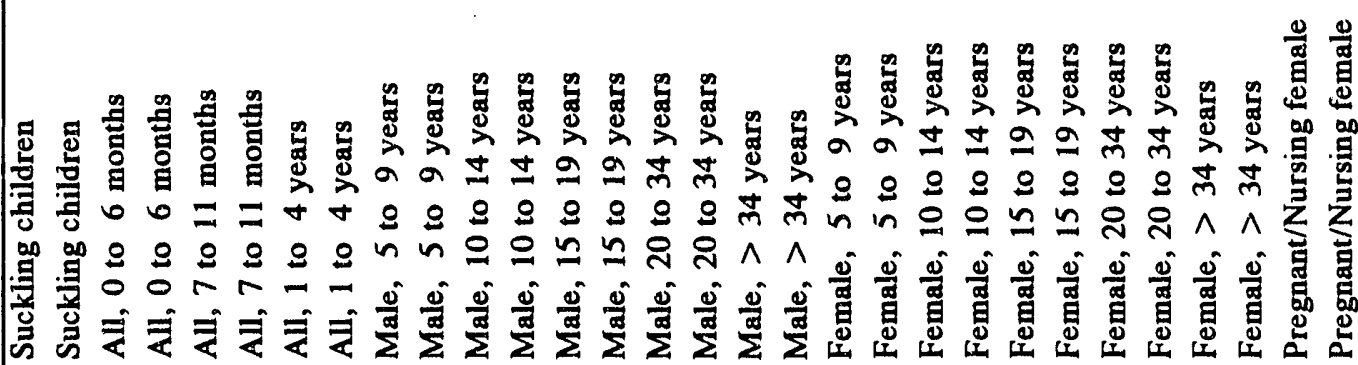 \\
\hline
\end{tabular}




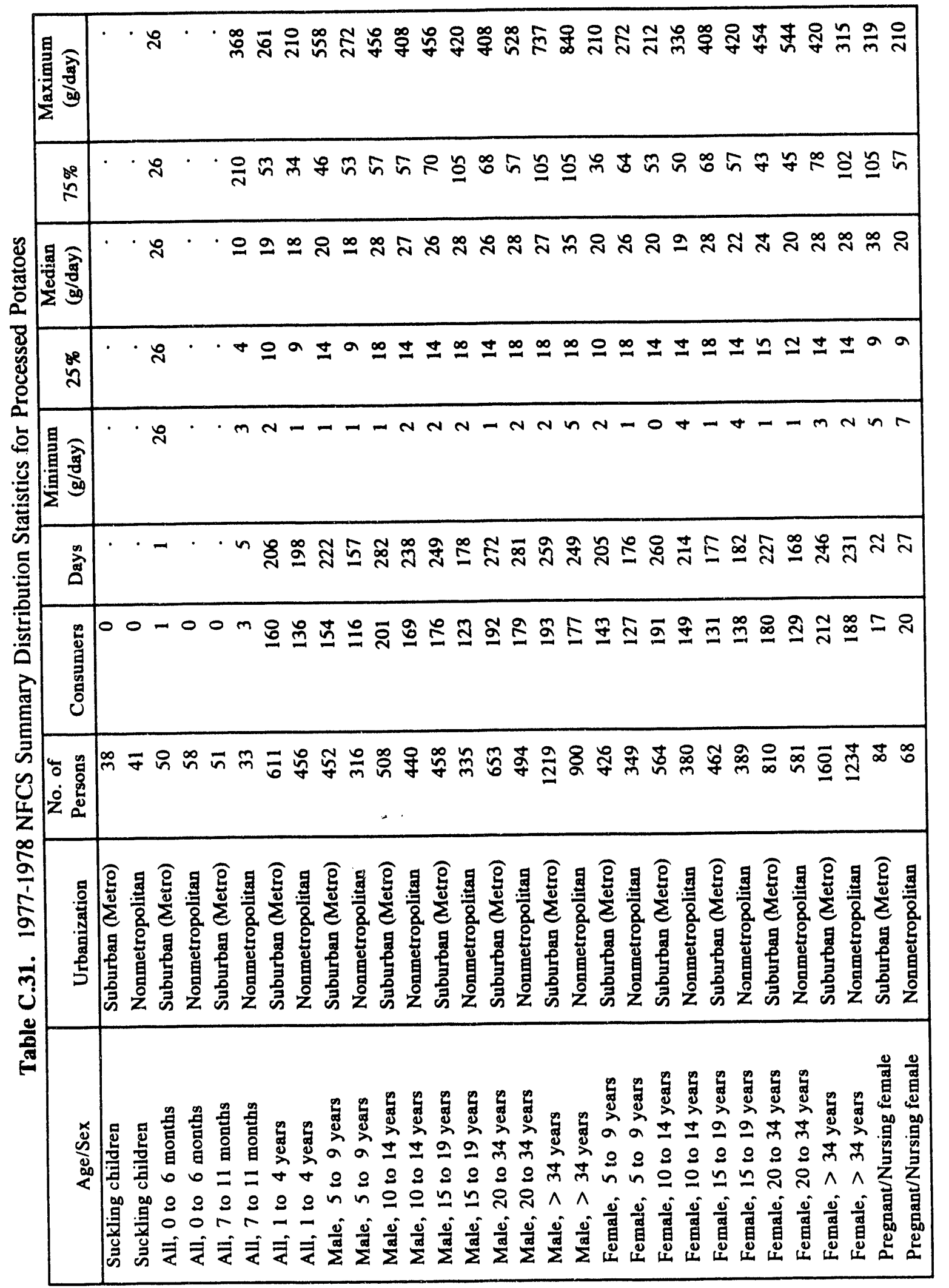

C. 32 


\begin{tabular}{|c|c|}
\hline$\frac{1}{5}$ & 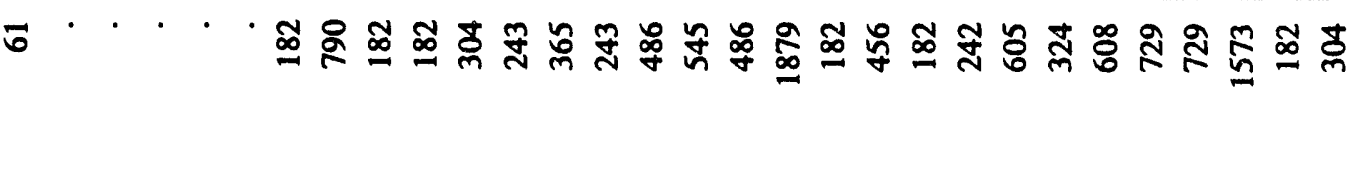 \\
\hline$\stackrel{8}{2}$ & 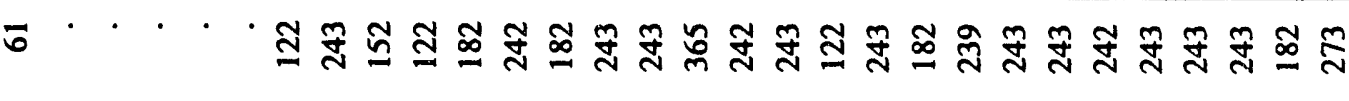 \\
\hline 迹 & 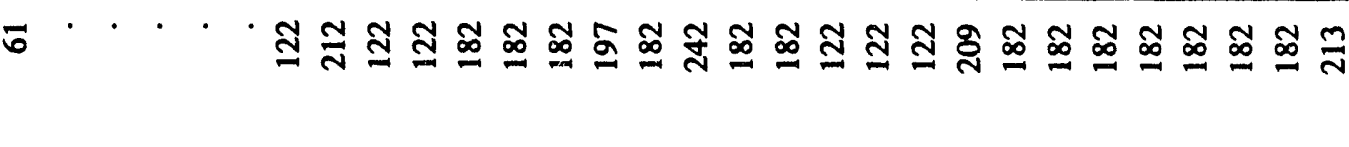 \\
\hline$\ddot{z}$ & 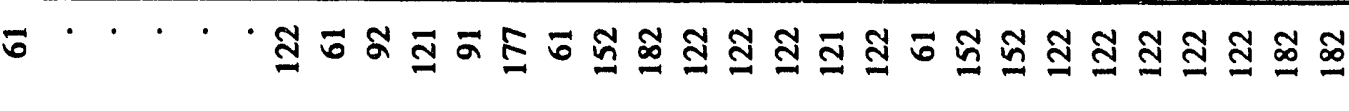 \\
\hline 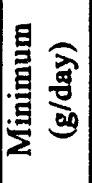 & 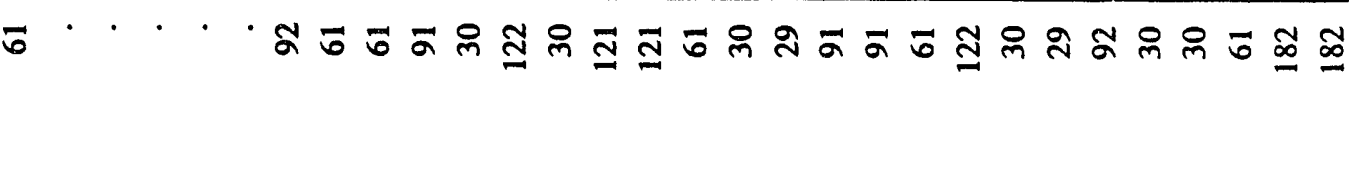 \\
\hline$\stackrel{\mathrm{n}}{\tilde{\mathrm{a}}}$ & 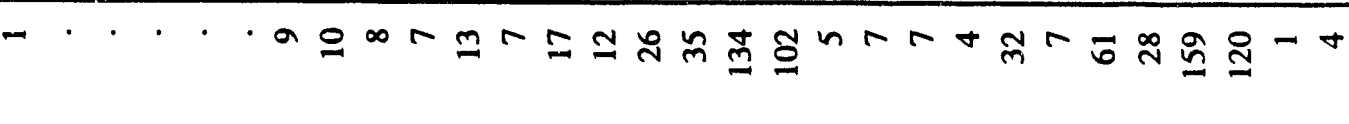 \\
\hline 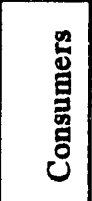 & - 00000 ON \\
\hline 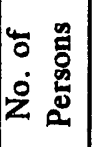 & 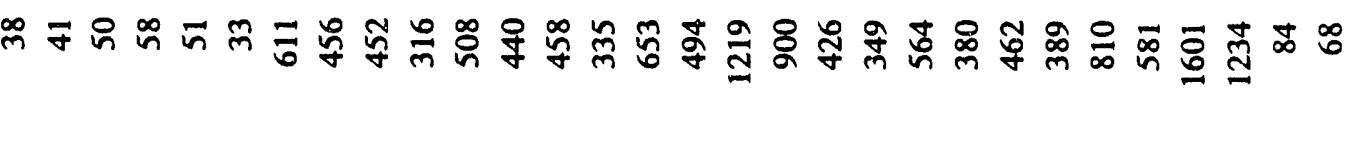 \\
\hline & 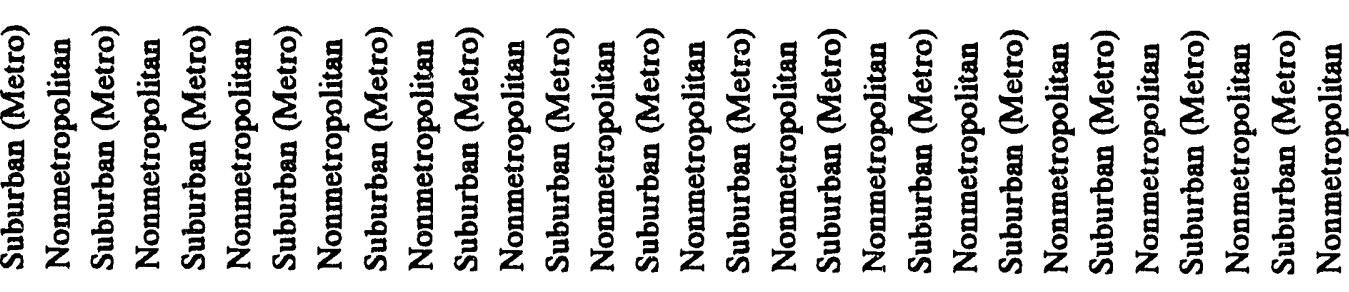 \\
\hline & 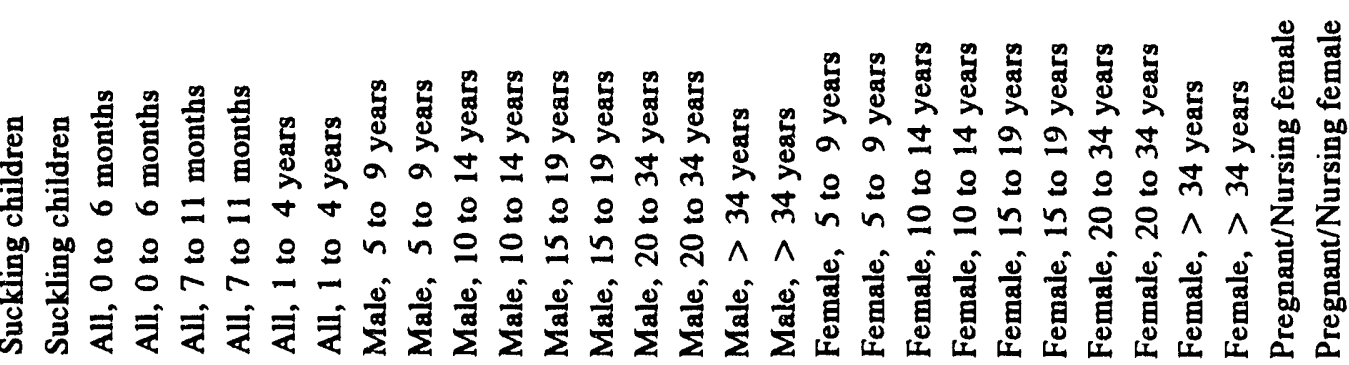 \\
\hline
\end{tabular}




\begin{tabular}{|c|c|}
\hline 量 & 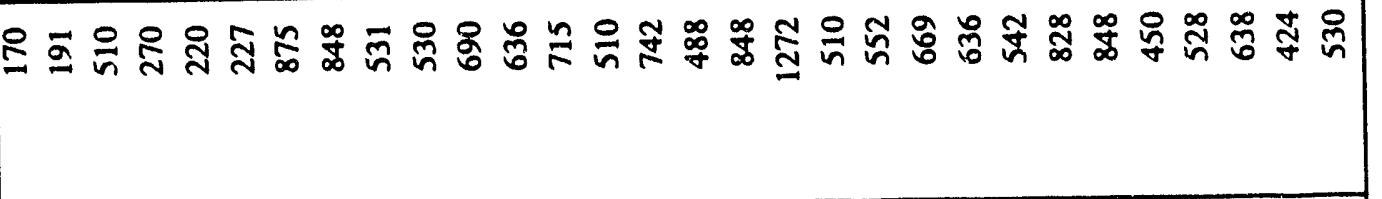 \\
\hline 8 & 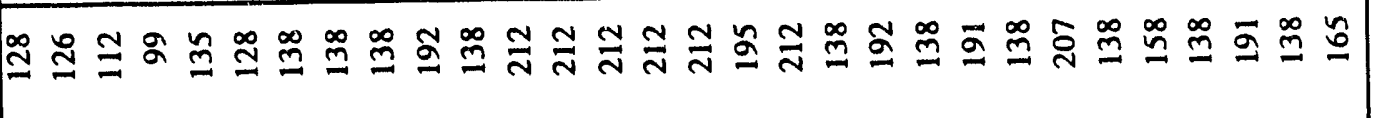 \\
\hline 通 & 요 \\
\hline$y$ & t ర \\
\hline 量拿 & 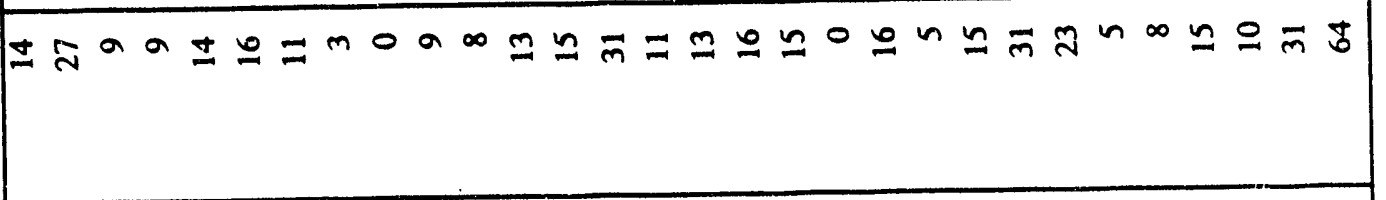 \\
\hline$\stackrel{n}{\mathrm{~m}}$ & స ส \\
\hline ن & "0 \\
\hline 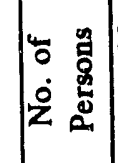 & 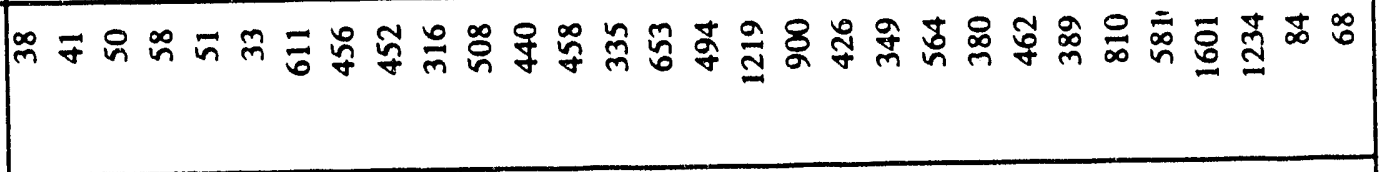 \\
\hline 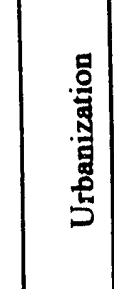 & 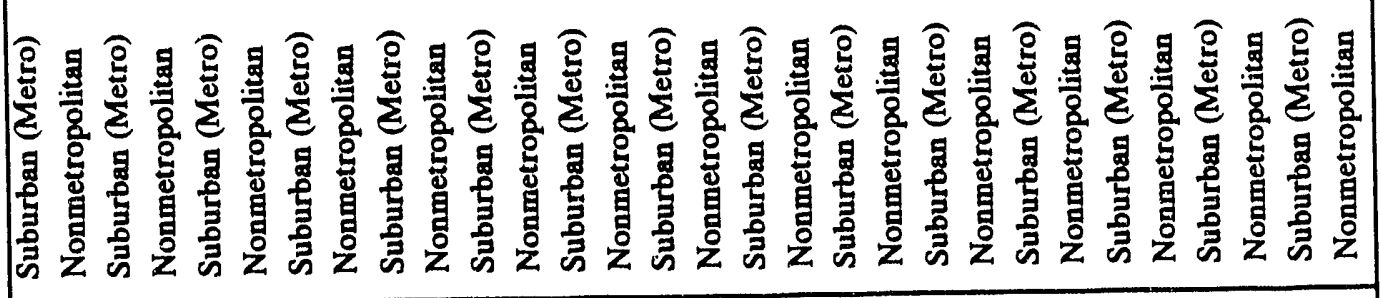 \\
\hline 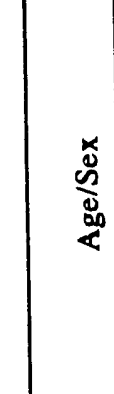 & 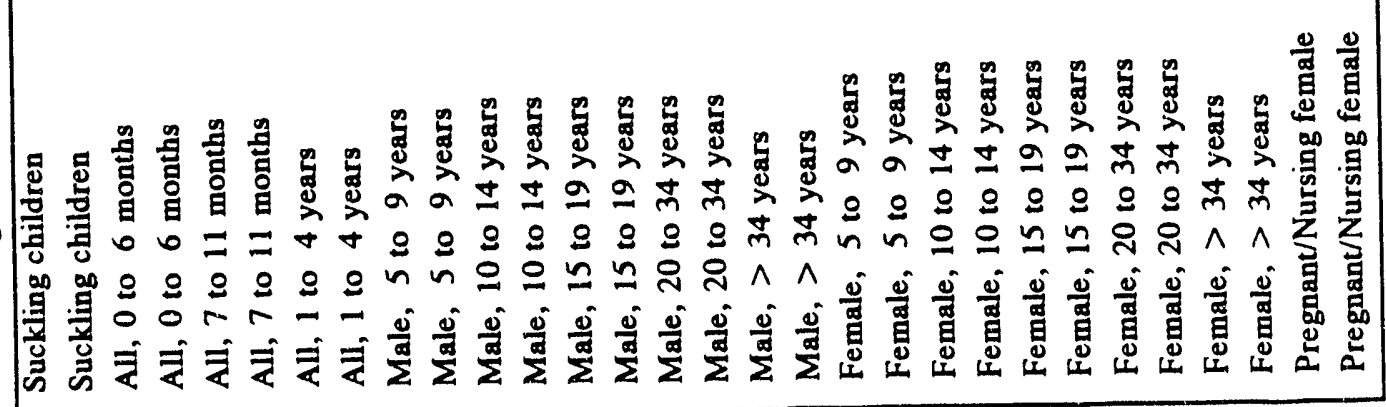 \\
\hline
\end{tabular}




\begin{tabular}{|c|c|}
\hline 量䇏 & 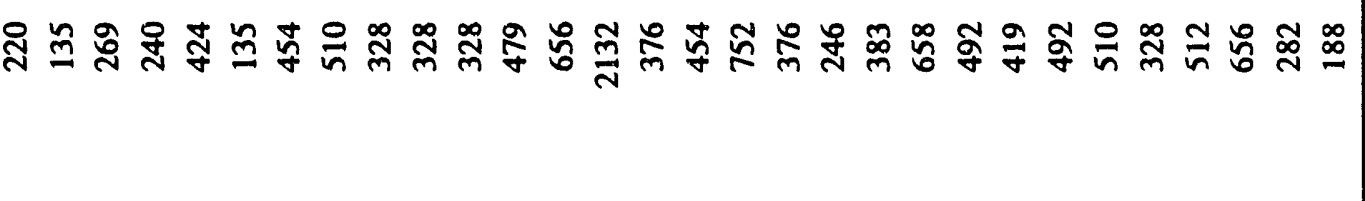 \\
\hline 象 & 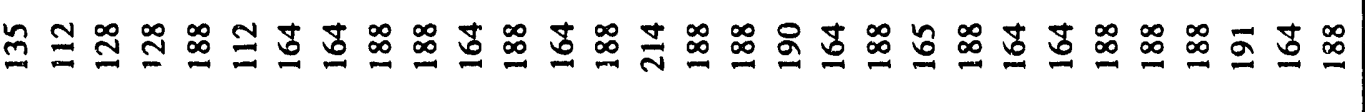 \\
\hline$\frac{5}{2} \frac{3}{3}$ & 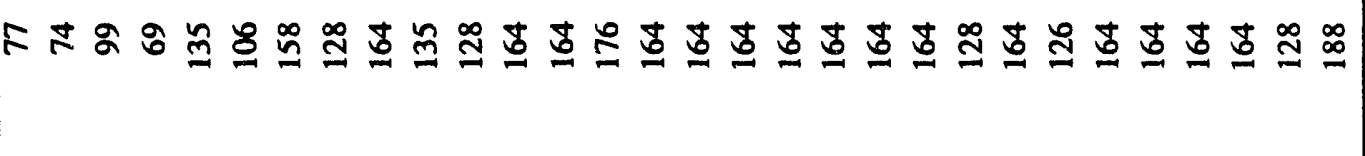 \\
\hline åd & 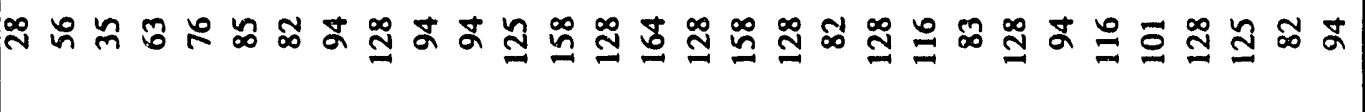 \\
\hline$\frac{1}{2}$ & 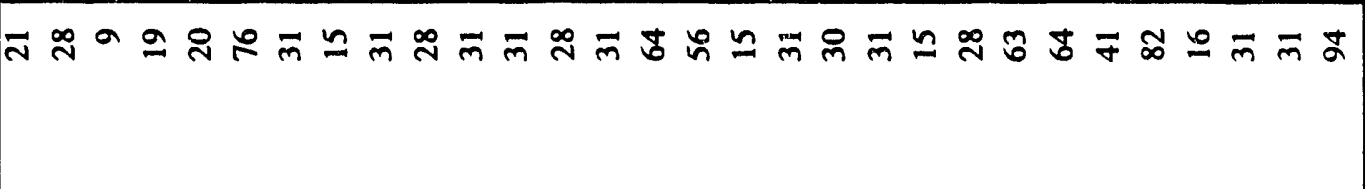 \\
\hline 鮯 & 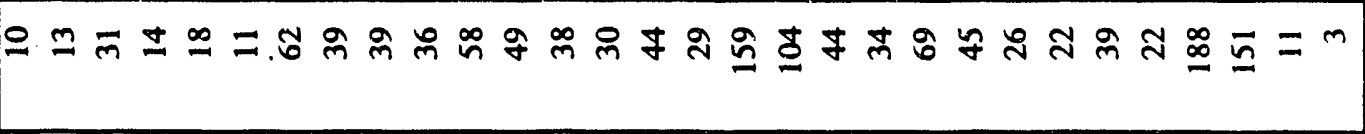 \\
\hline & 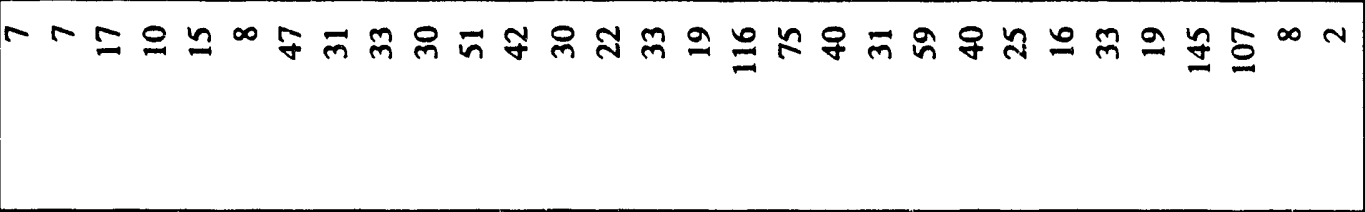 \\
\hline 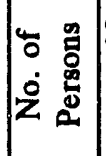 & 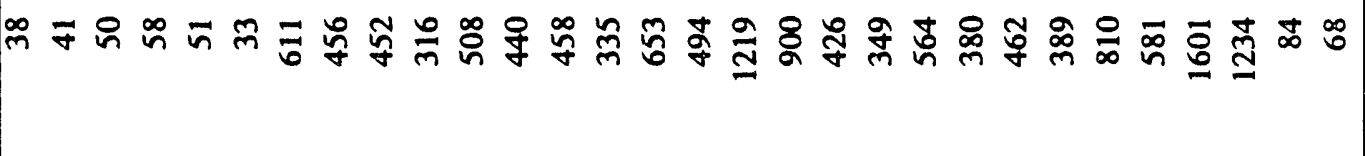 \\
\hline & 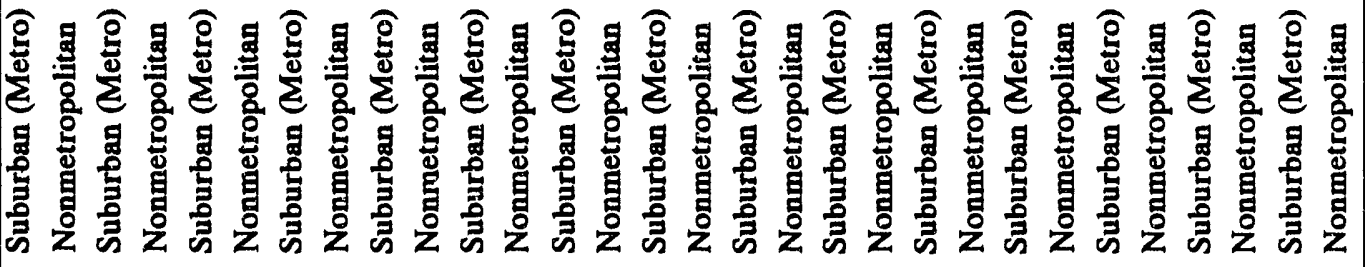 \\
\hline & 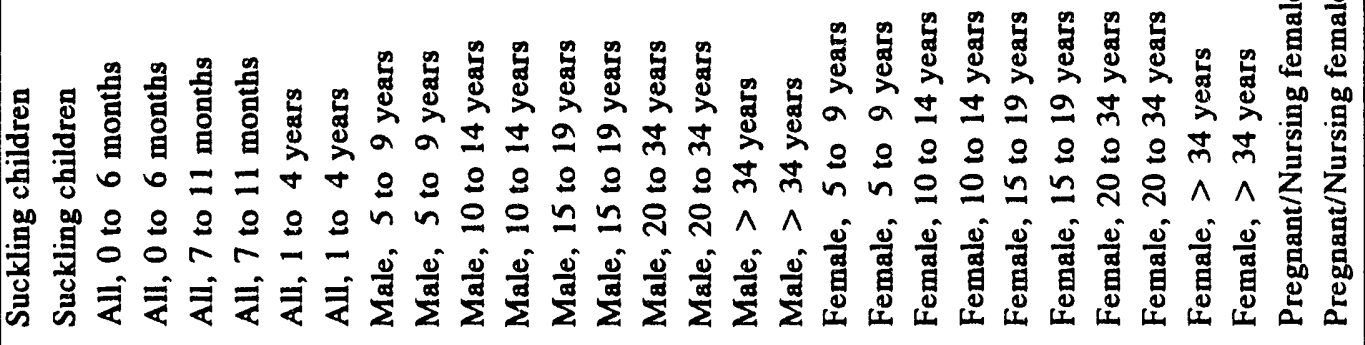 \\
\hline
\end{tabular}




\begin{tabular}{|c|c|}
\hline 要票 & 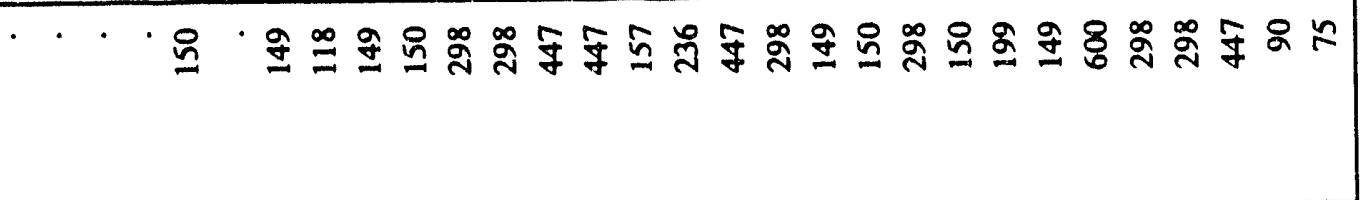 \\
\hline $\begin{array}{l}8 \\
\Sigma \\
\Sigma\end{array}$ & 员: \\
\hline$\sum \frac{9}{3}$ & 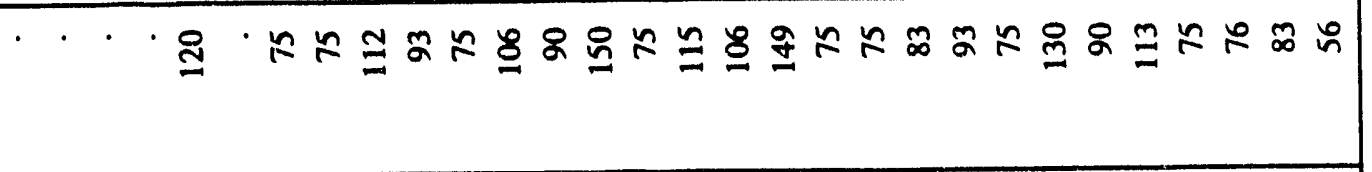 \\
\hline$\ddot{n}$ & 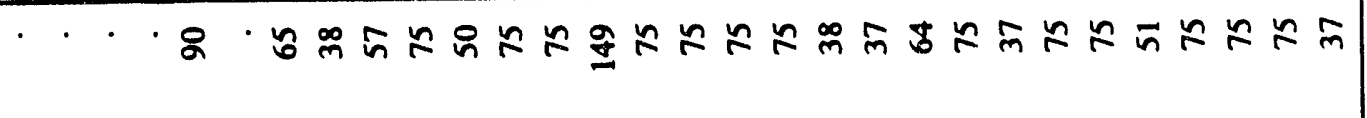 \\
\hline$\frac{1}{3}$ & 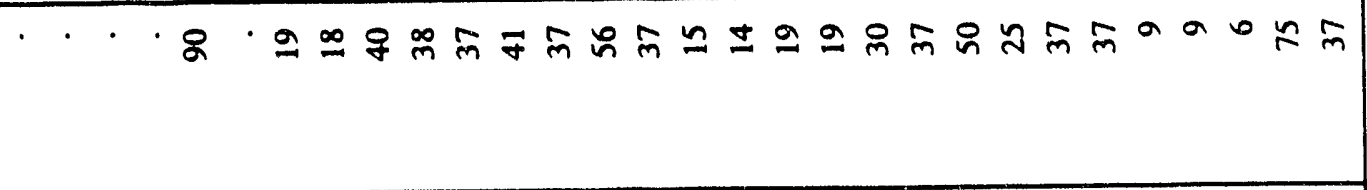 \\
\hline$\stackrel{n}{a}$ & 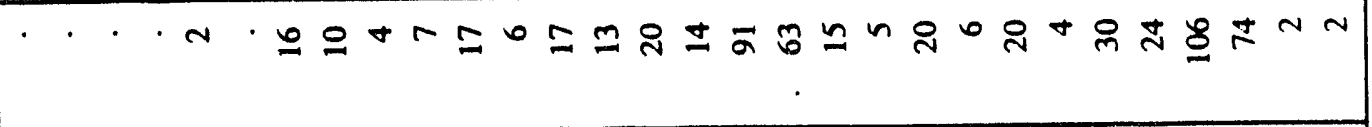 \\
\hline 要 & 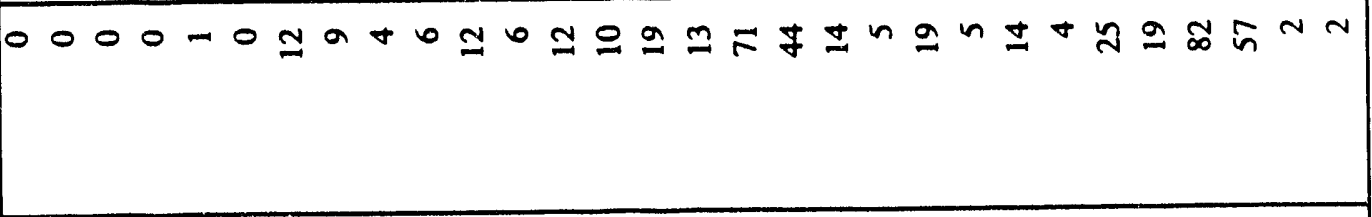 \\
\hline 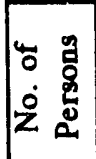 & 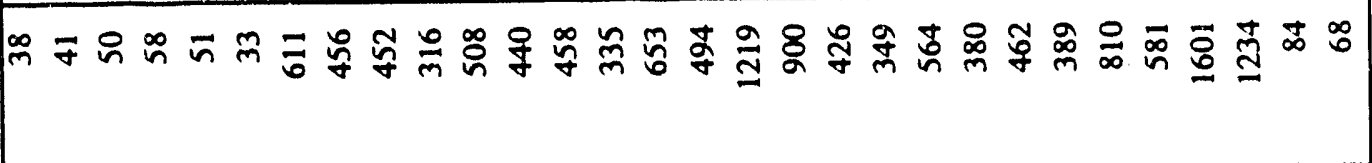 \\
\hline 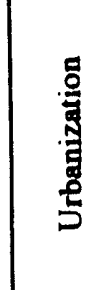 & 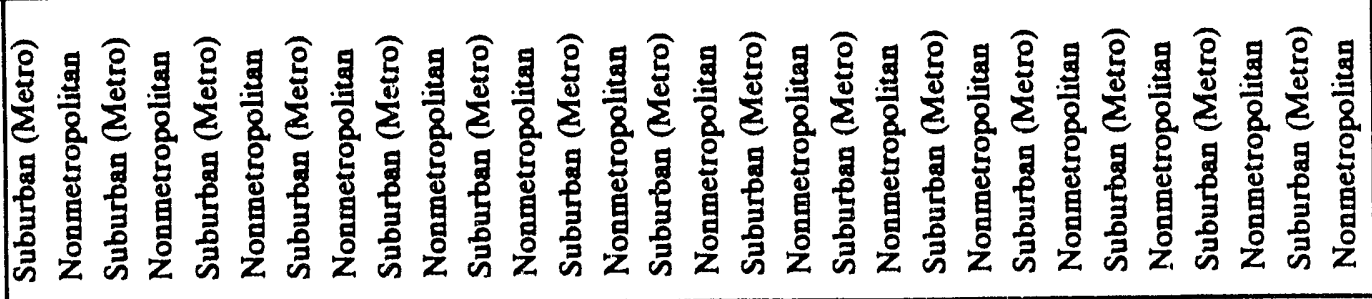 \\
\hline & 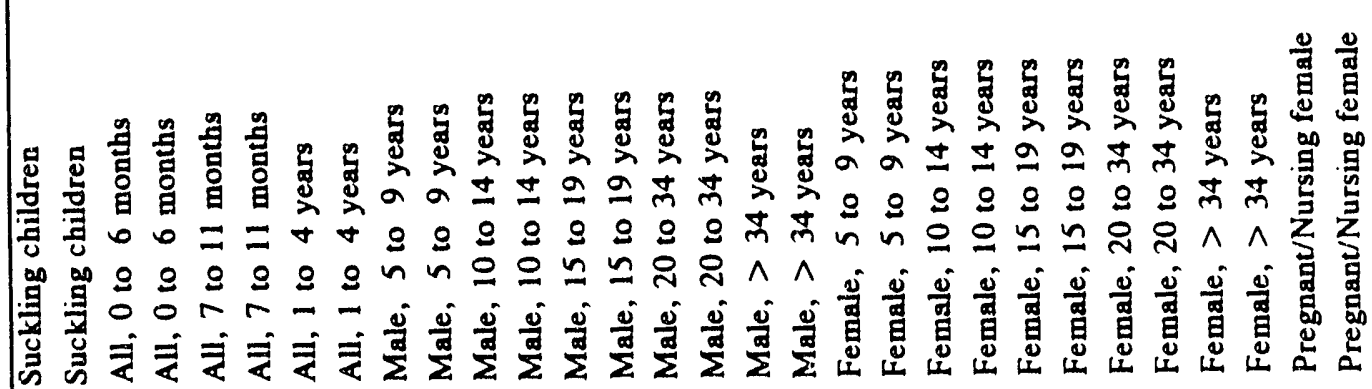 \\
\hline
\end{tabular}

C. 36 


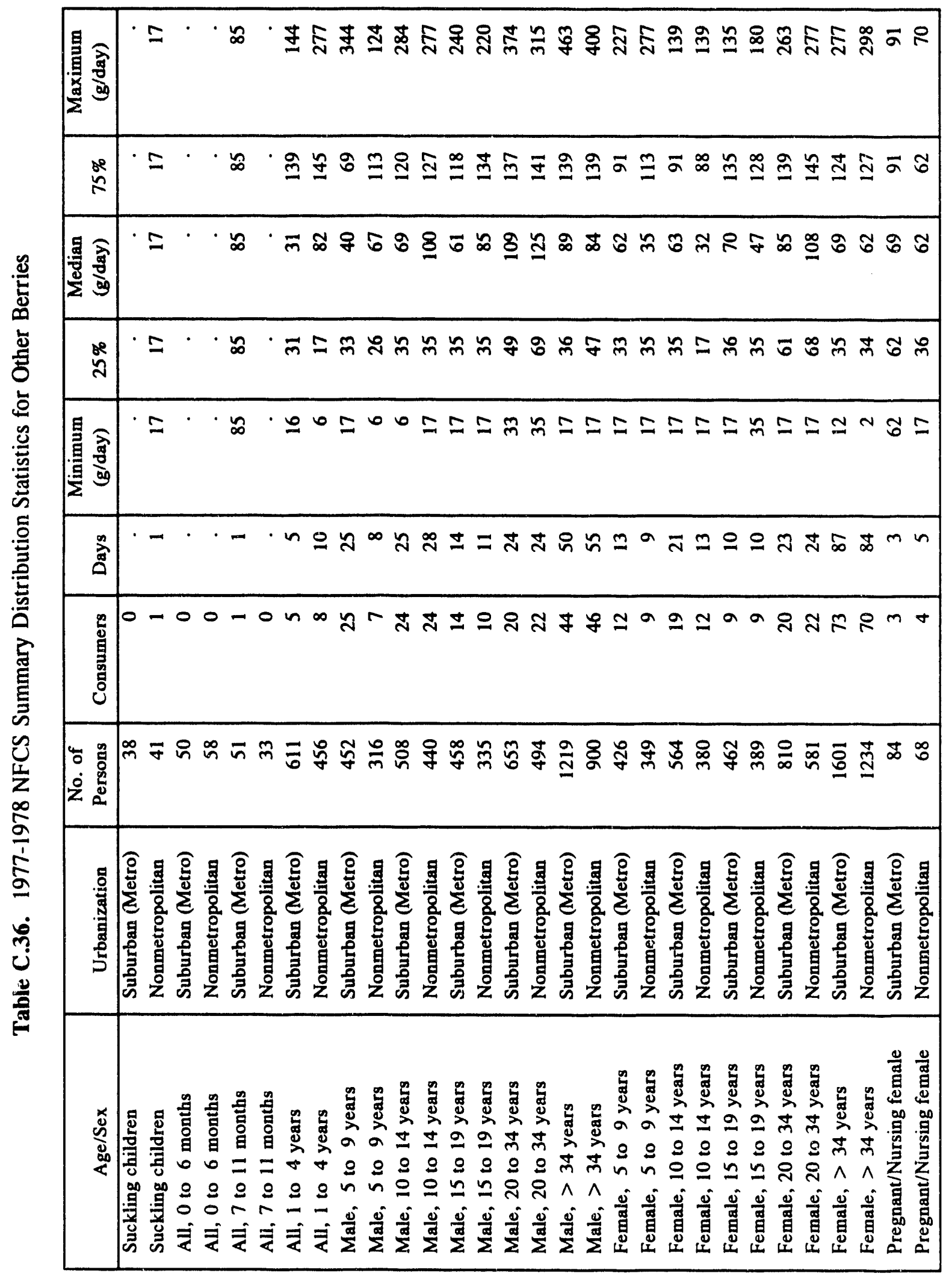




\begin{tabular}{|c|c|}
\hline 量 & 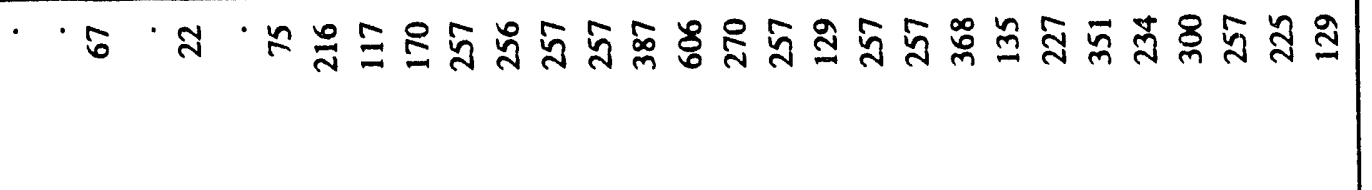 \\
\hline$\stackrel{8}{2}$ & 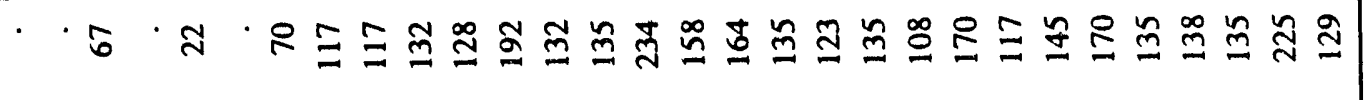 \\
\hline 递要 & 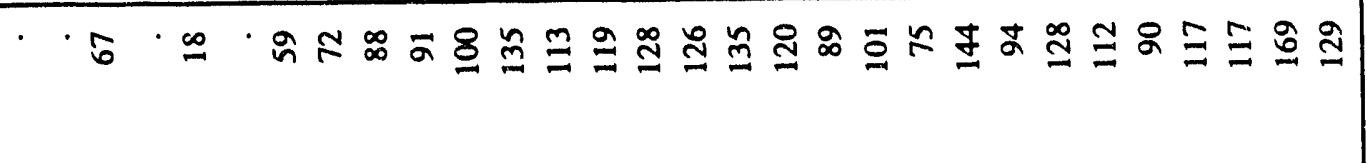 \\
\hline$y^{2}$ & 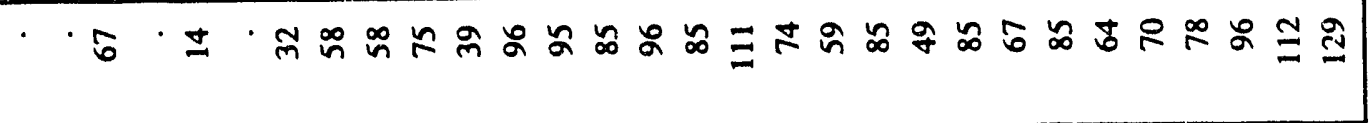 \\
\hline 量 & 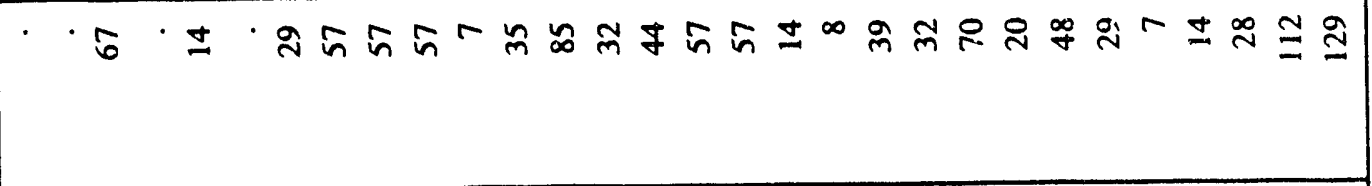 \\
\hline$\stackrel{m}{\Delta}$ & 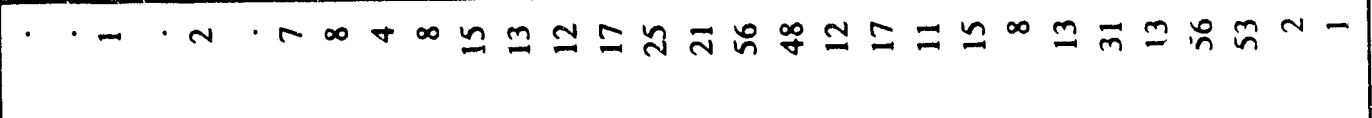 \\
\hline 䇋 & 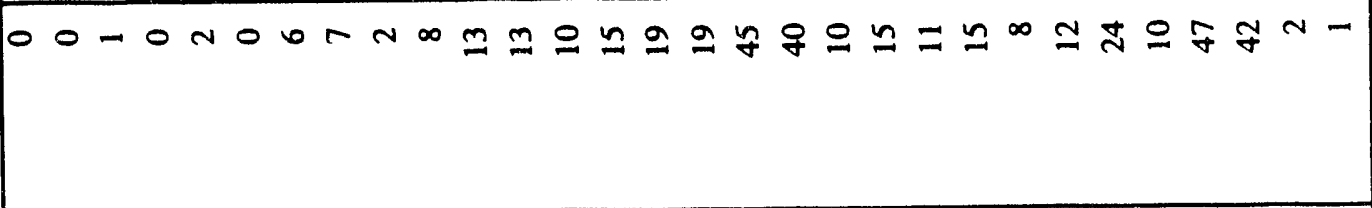 \\
\hline $\begin{array}{ll}4 & 0 \\
0 & 0 \\
0 & 0 \\
0 & 0 \\
2\end{array}$ & 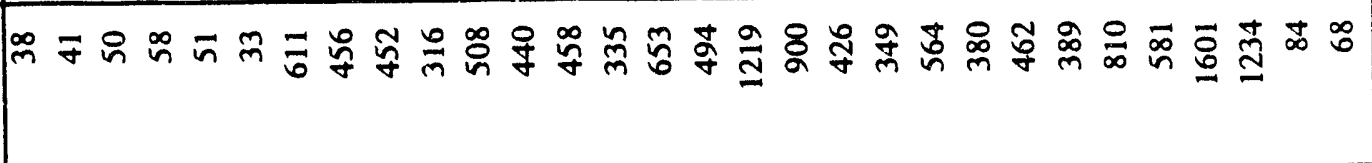 \\
\hline 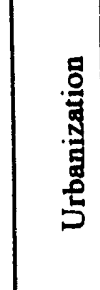 & 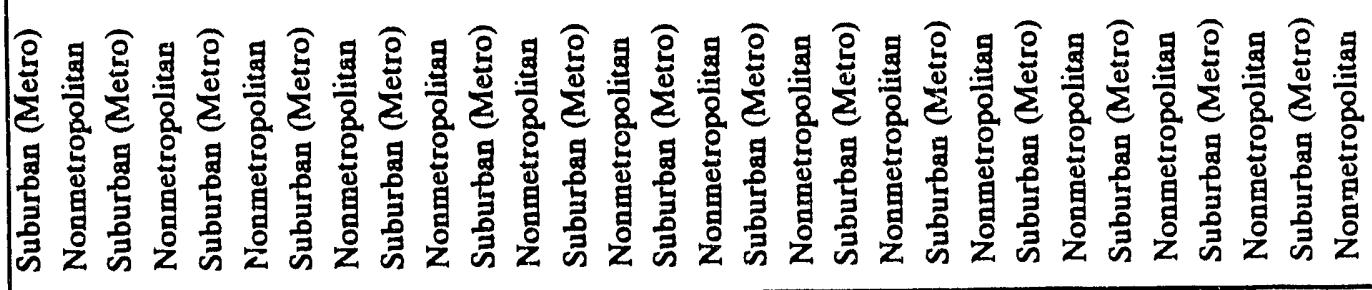 \\
\hline 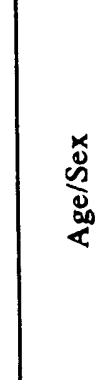 & 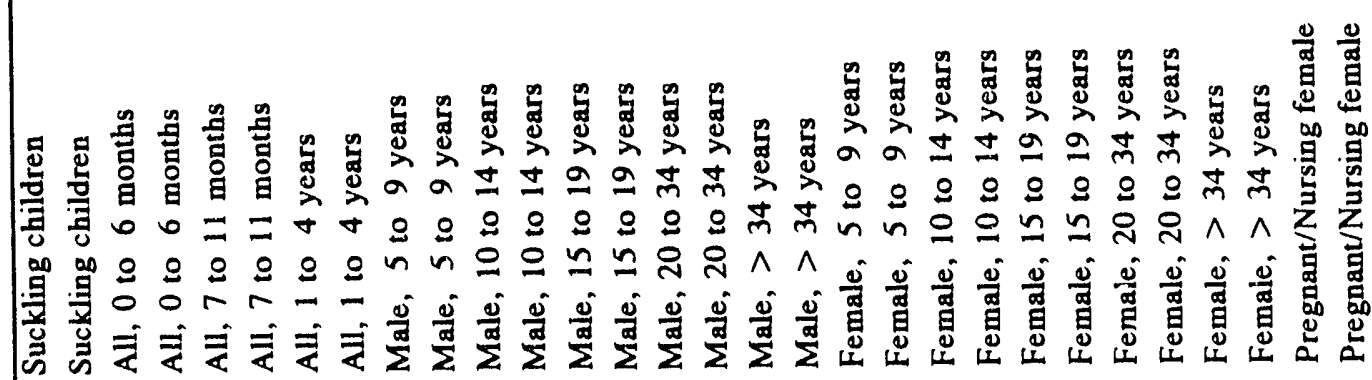 \\
\hline
\end{tabular}




\begin{tabular}{|c|c|}
\hline 爱 & 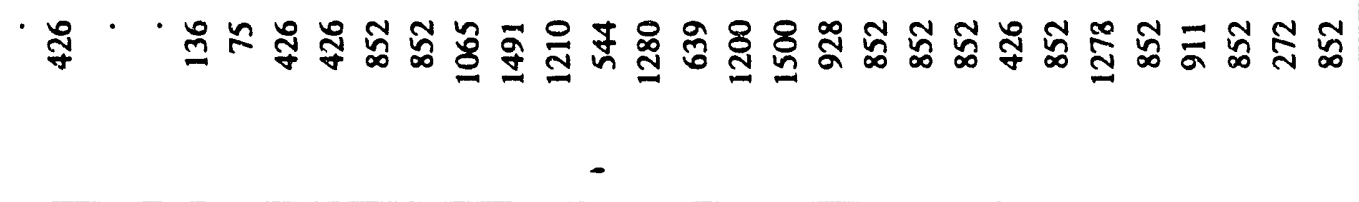 \\
\hline$\stackrel{80}{5}$ & 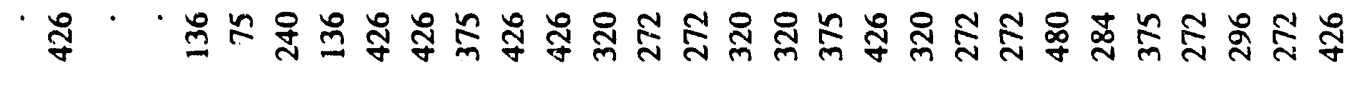 \\
\hline$\sum_{0}^{\frac{2}{2}}$ & 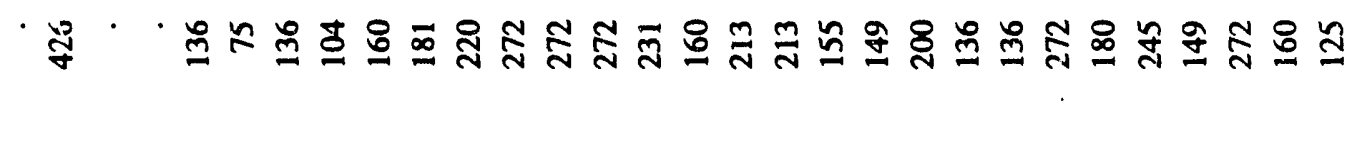 \\
\hline$y^{\circ}$ & 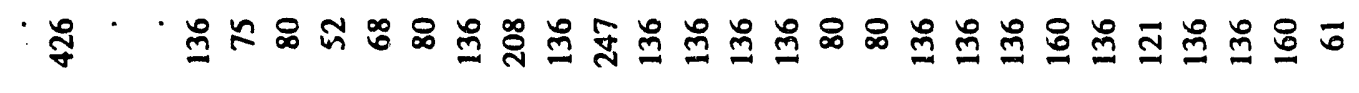 \\
\hline$\frac{1}{2}$ & 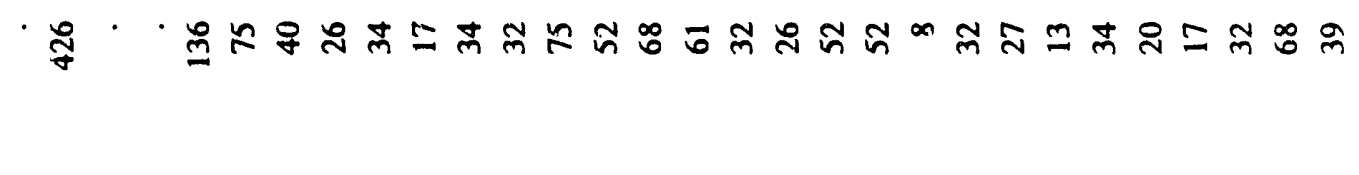 \\
\hline$\stackrel{\mathrm{m}}{\mathrm{a}}$ & 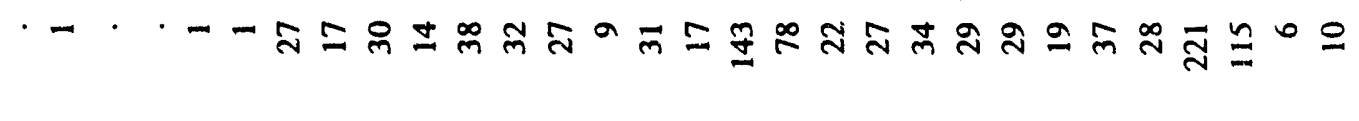 \\
\hline 递 & 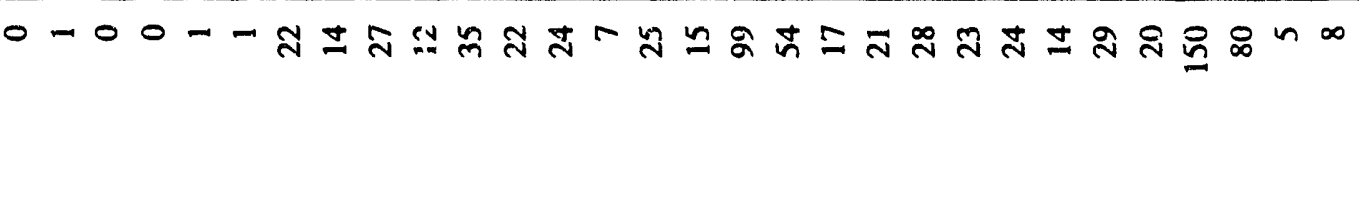 \\
\hline 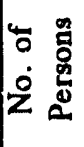 & 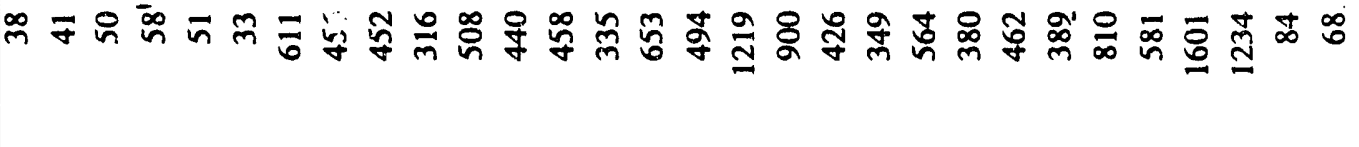 \\
\hline 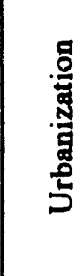 & 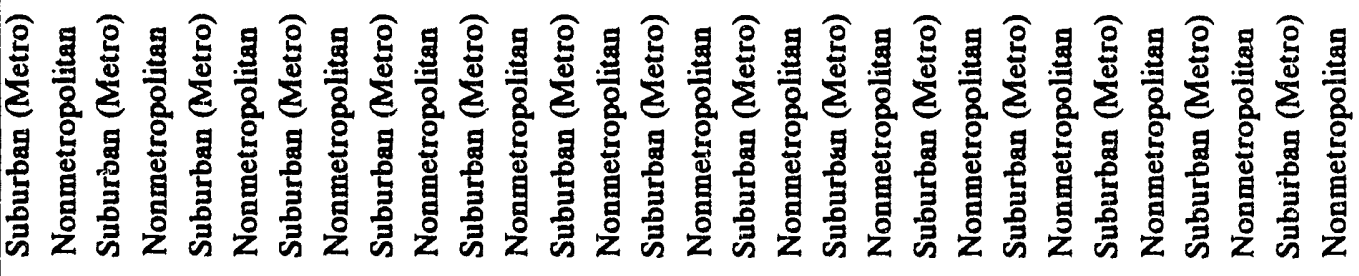 \\
\hline & 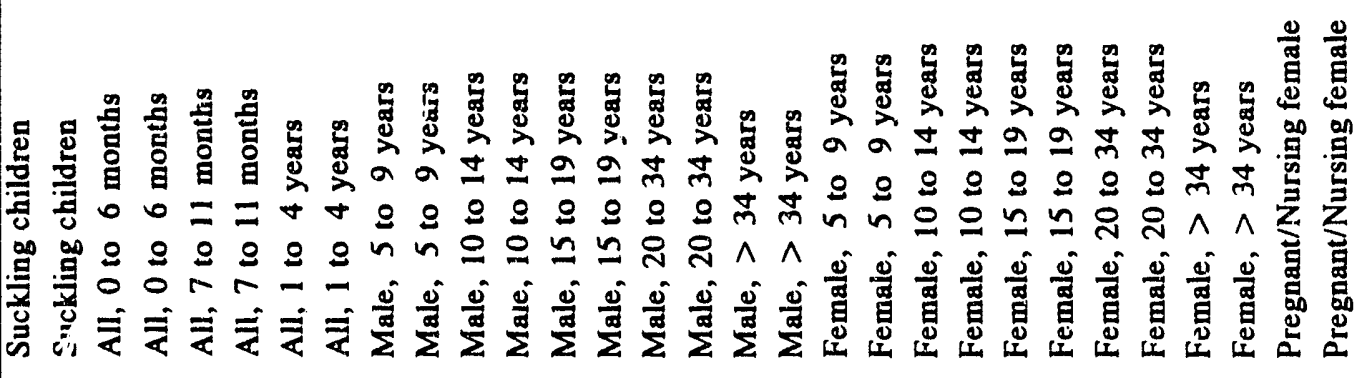 \\
\hline
\end{tabular}




\begin{tabular}{|c|c|}
\hline 量量 & 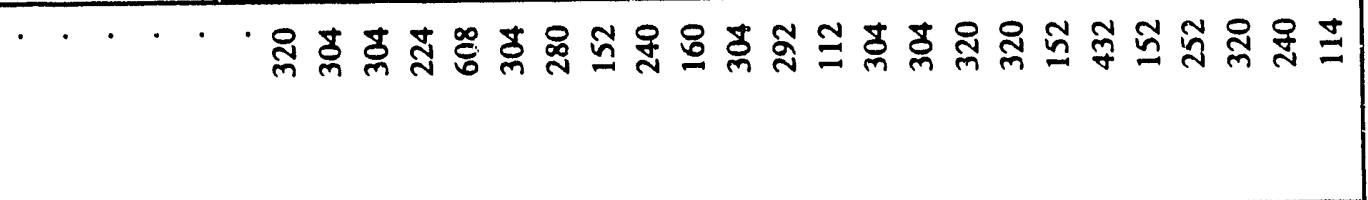 \\
\hline$\stackrel{8}{i}$ & 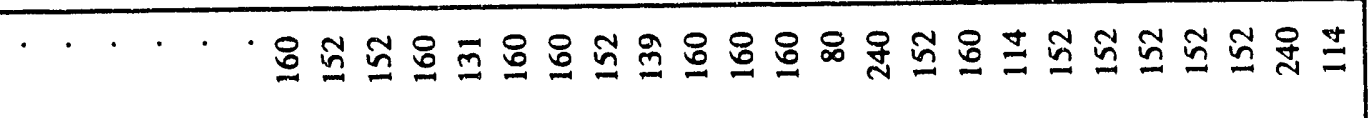 \\
\hline 造 & $\cdot \cdot \cdot \cdot \cdots \infty$ \\
\hline$y^{*}$ & 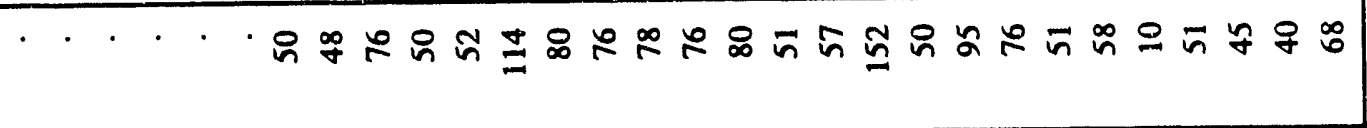 \\
\hline 要 & $\cdot \cdot \cdot \cdot \cdot \backsim \infty$ ․ \\
\hline 离 & 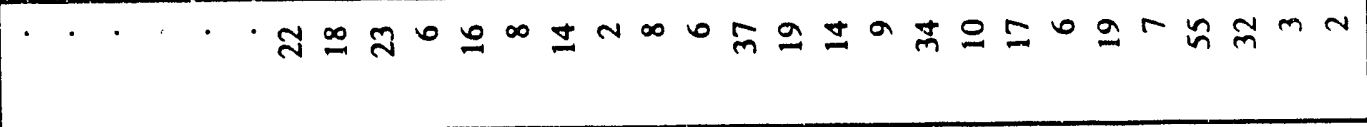 \\
\hline 兽 & 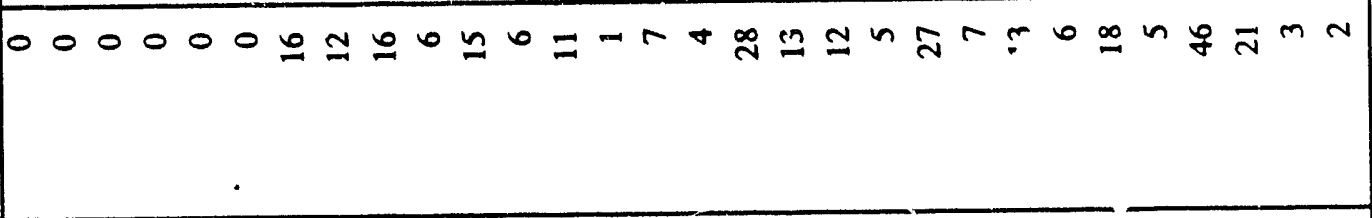 \\
\hline 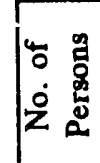 & 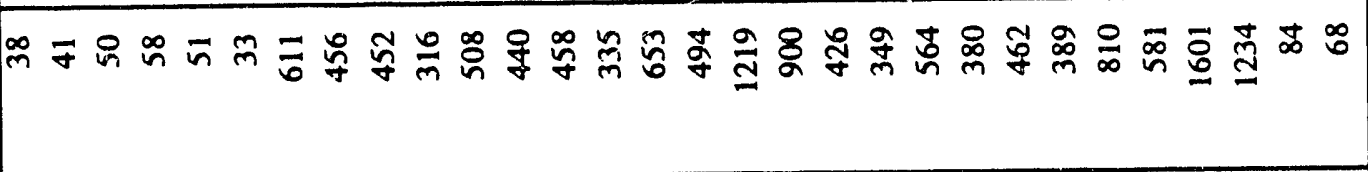 \\
\hline 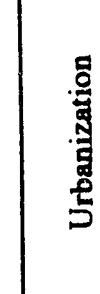 & 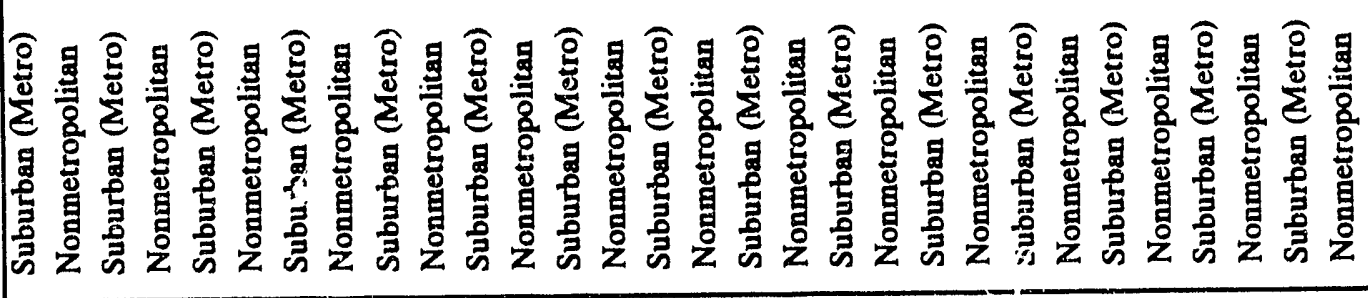 \\
\hline 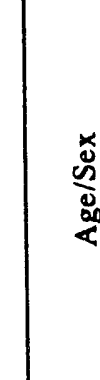 & 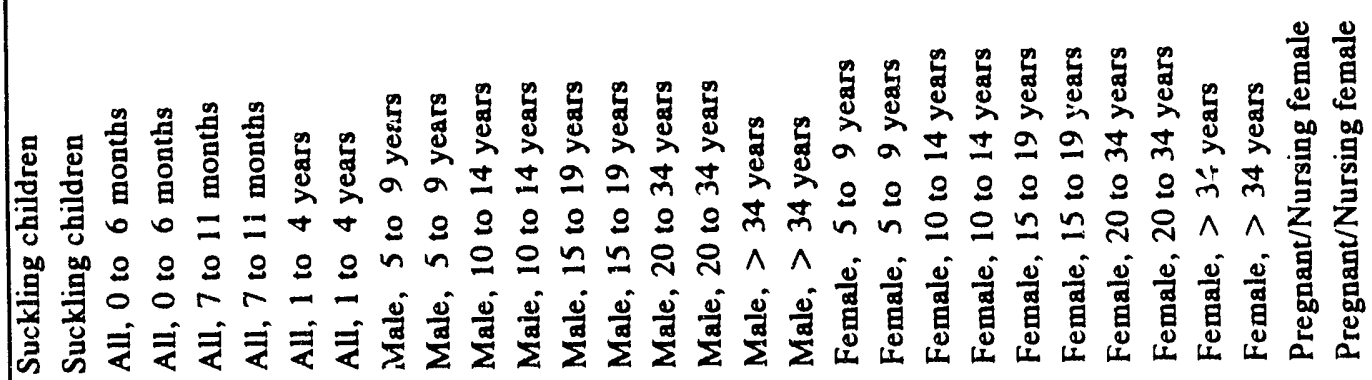 \\
\hline
\end{tabular}

C. 40 


\begin{tabular}{|c|c|}
\hline 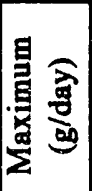 & 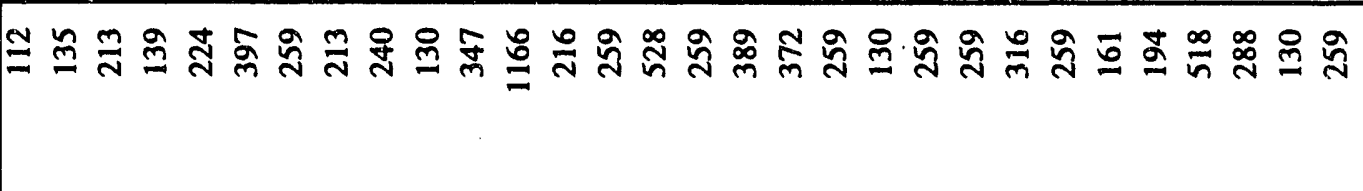 \\
\hline i̊ & 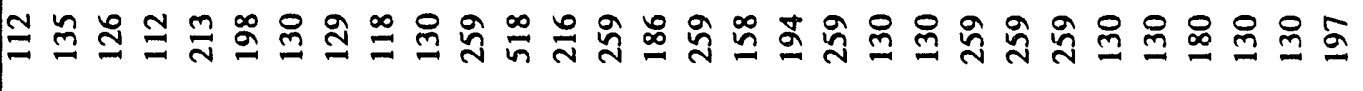 \\
\hline$\frac{5}{5}$ & 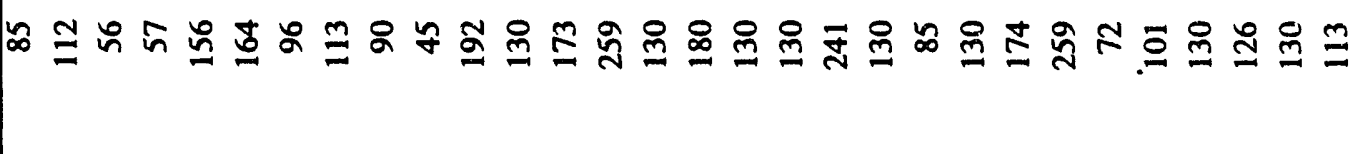 \\
\hline$\ddot{z}$ & 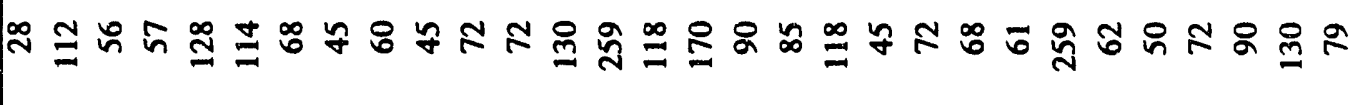 \\
\hline$\frac{1}{\frac{5}{5}}$ & 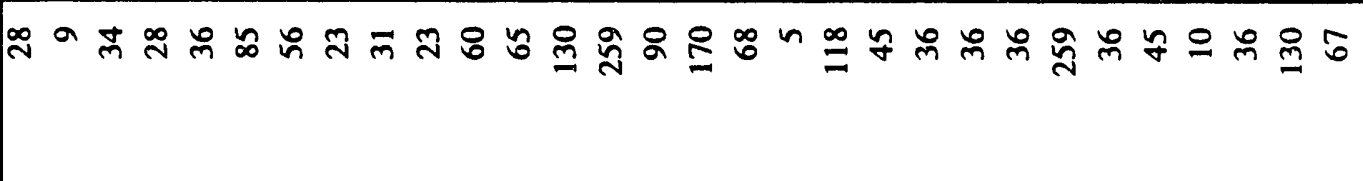 \\
\hline$\stackrel{\infty}{\mathrm{m}}$ & 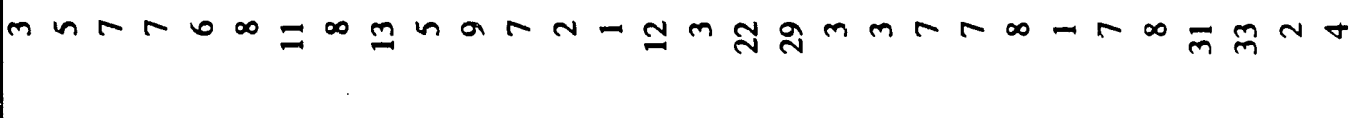 \\
\hline 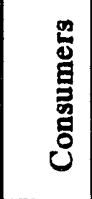 & 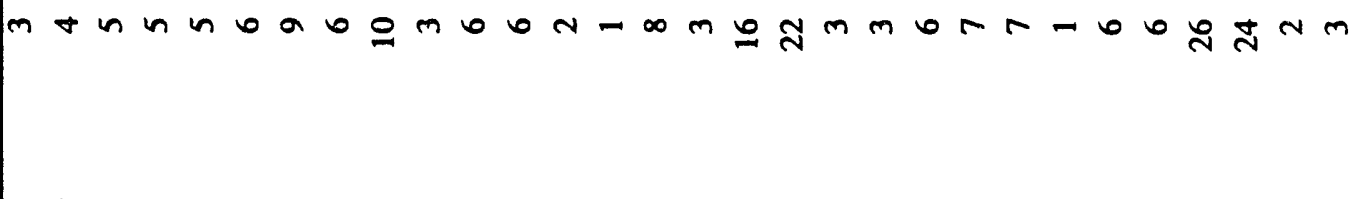 \\
\hline 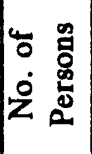 & 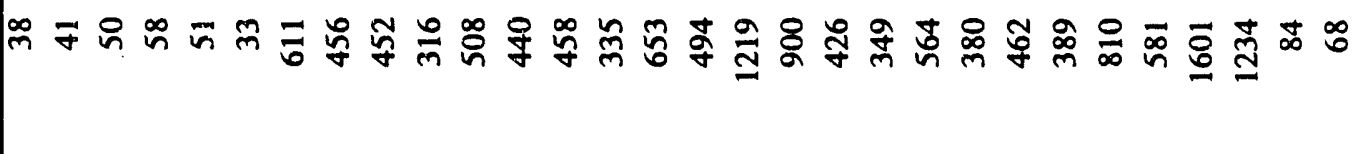 \\
\hline 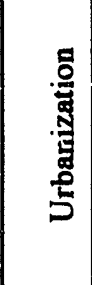 & 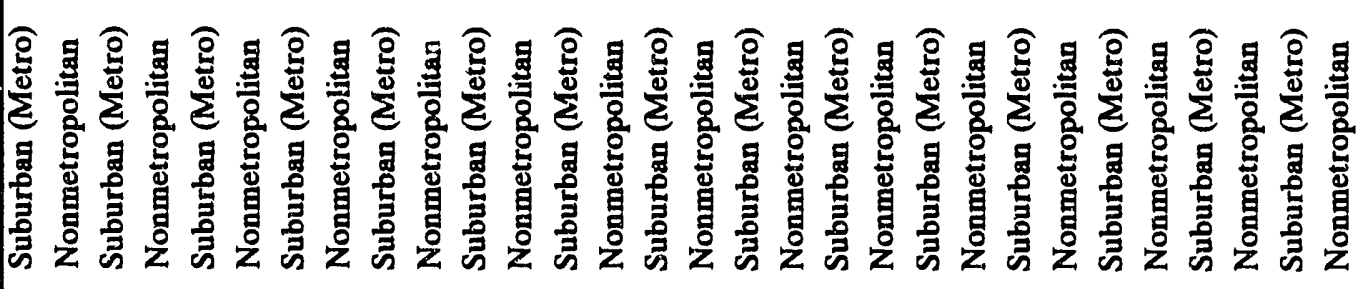 \\
\hline 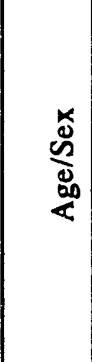 & 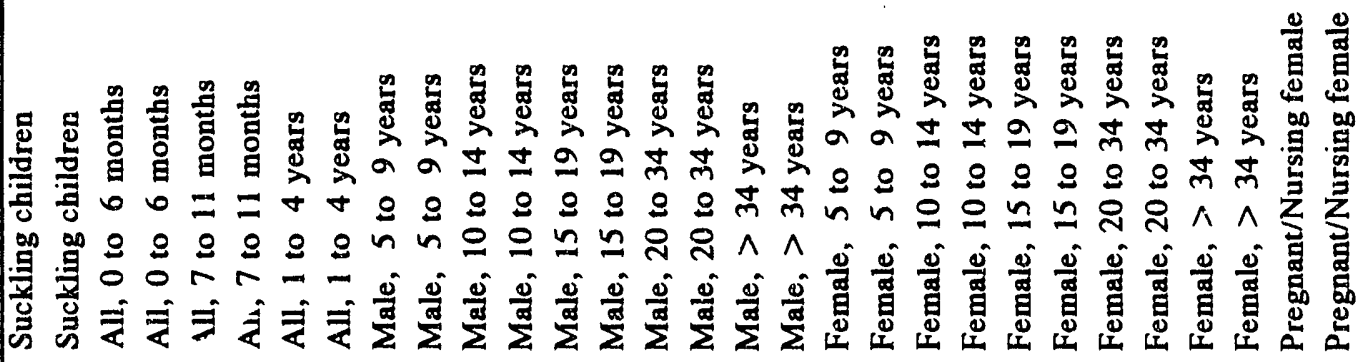 \\
\hline
\end{tabular}




\begin{tabular}{|c|c|}
\hline$\frac{1}{\frac{1}{6}}$ & 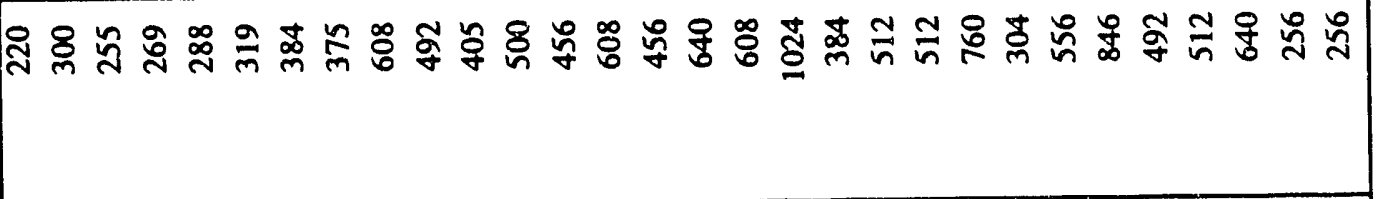 \\
\hline$\stackrel{8}{2}$ & 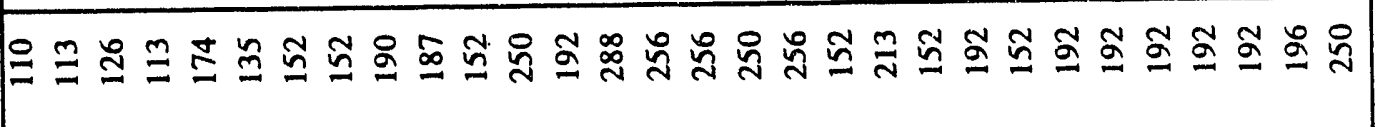 \\
\hline 通 & 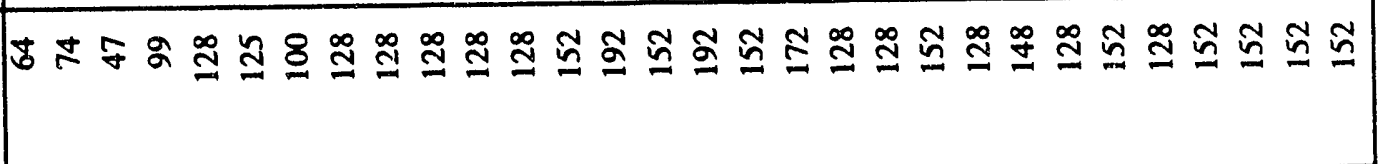 \\
\hline$\ddot{y}$ & 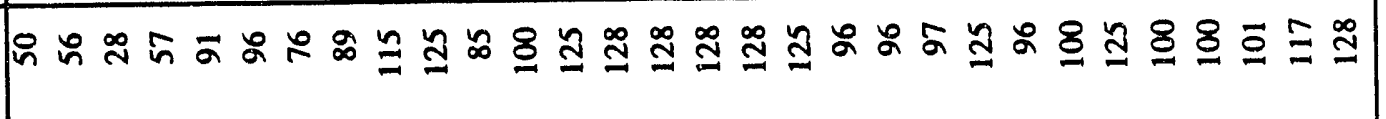 \\
\hline 量 & 2 \\
\hline$\stackrel{n}{\Delta}$ & అ \\
\hline 总 & 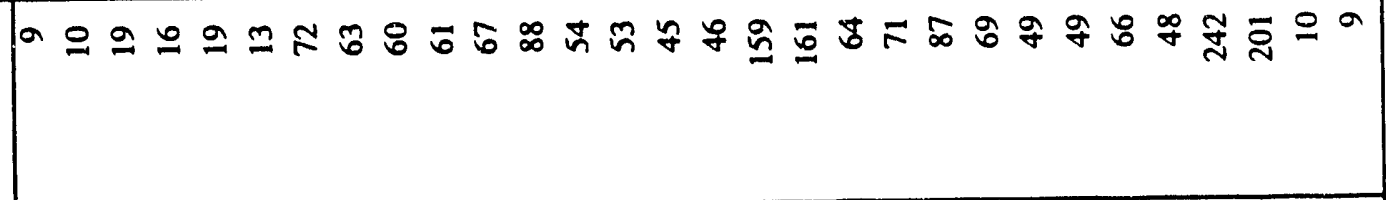 \\
\hline 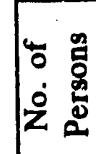 & 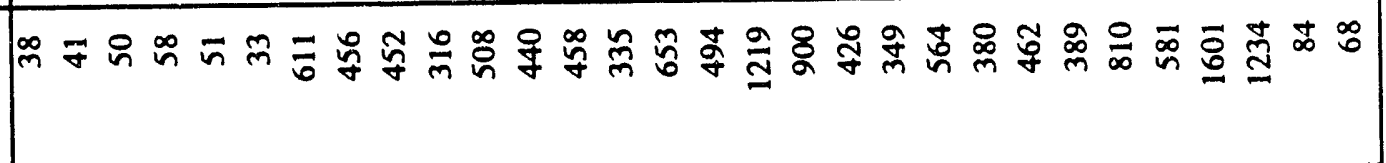 \\
\hline 兽 & 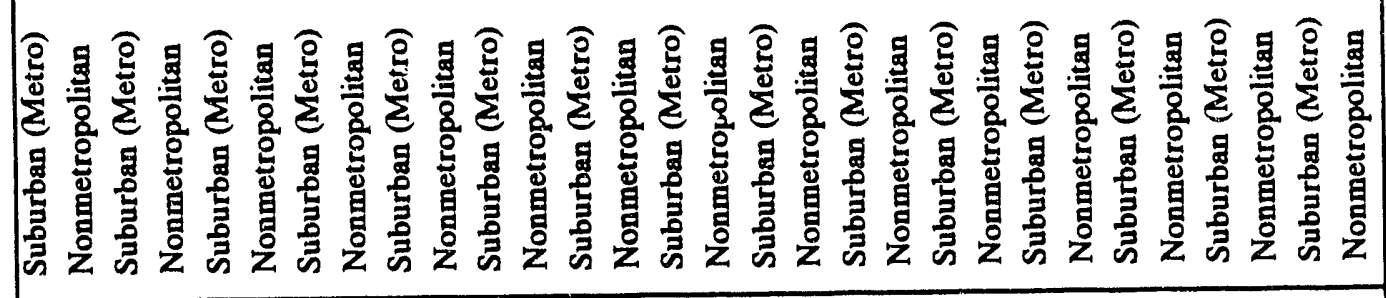 \\
\hline & 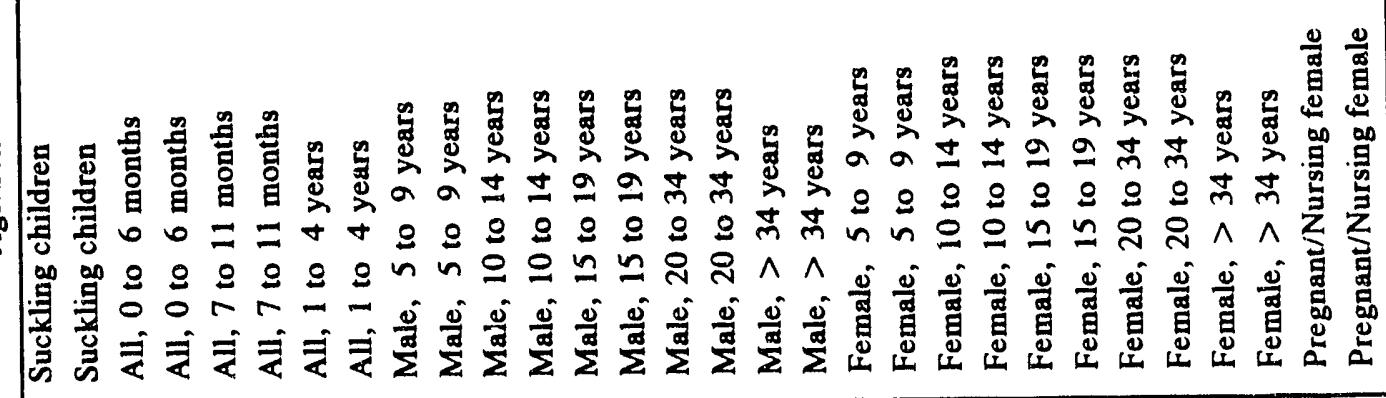 \\
\hline
\end{tabular}

C. 42 


\begin{tabular}{|c|c|}
\hline 要 & 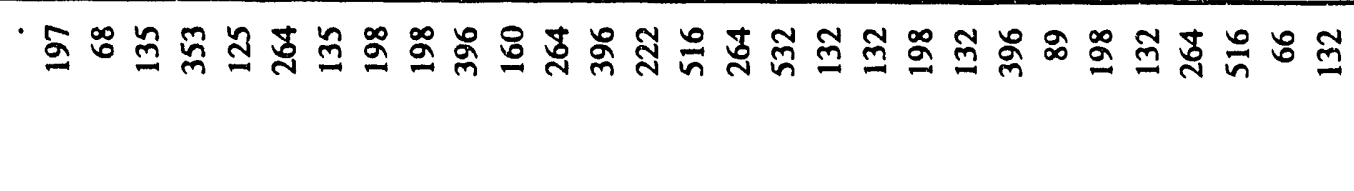 \\
\hline$\stackrel{80}{\check{2}}$ & 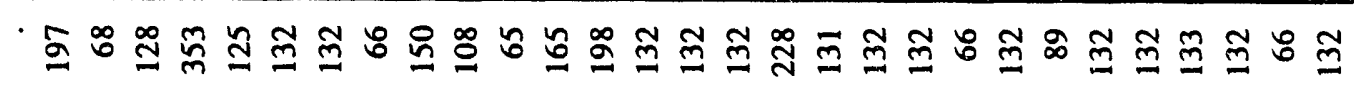 \\
\hline 通 & • \\
\hline$\stackrel{g}{*}$ & 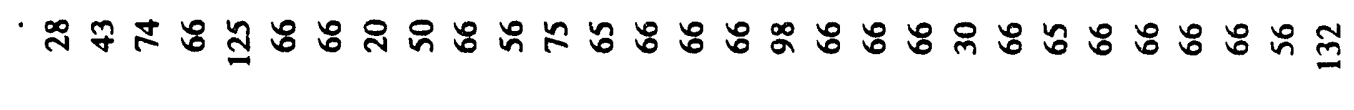 \\
\hline$\frac{1}{3}$ & 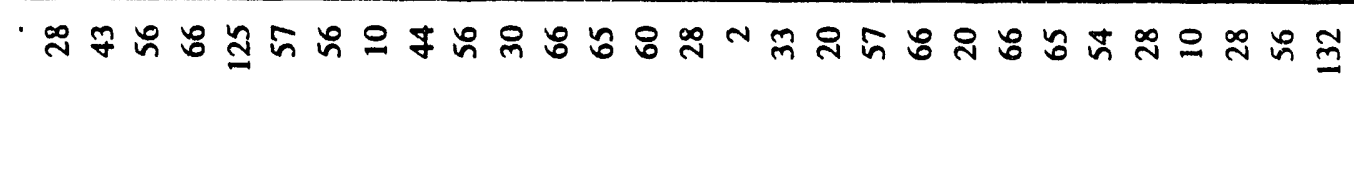 \\
\hline 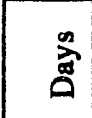 & 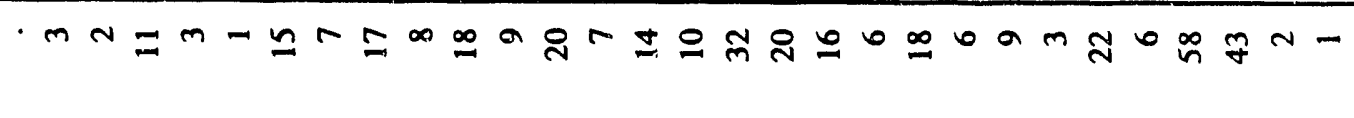 \\
\hline 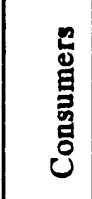 & 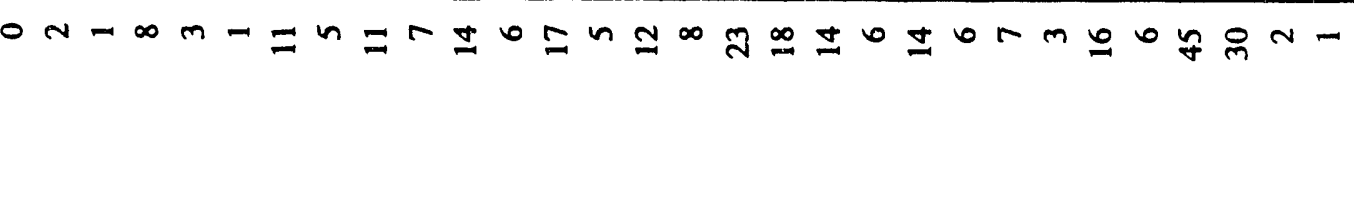 \\
\hline 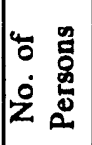 & 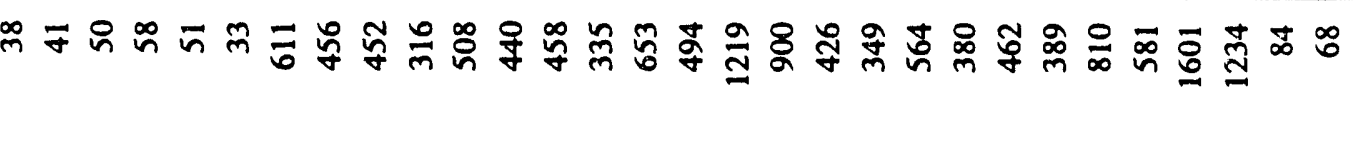 \\
\hline 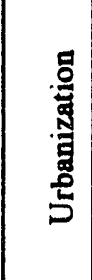 & 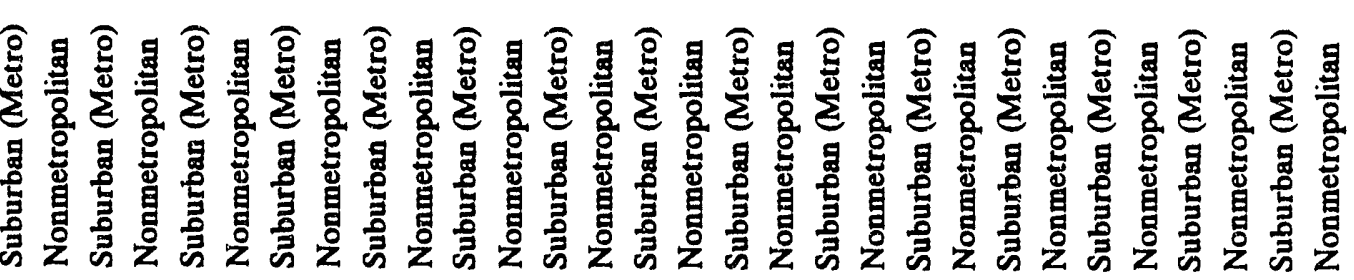 \\
\hline 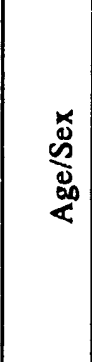 & 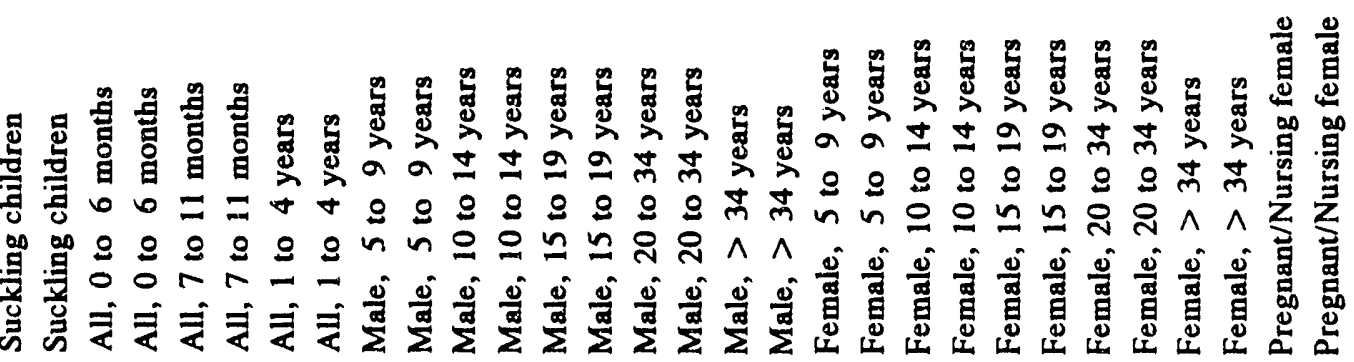 \\
\hline
\end{tabular}




\begin{tabular}{|c|c|}
\hline 要要 & 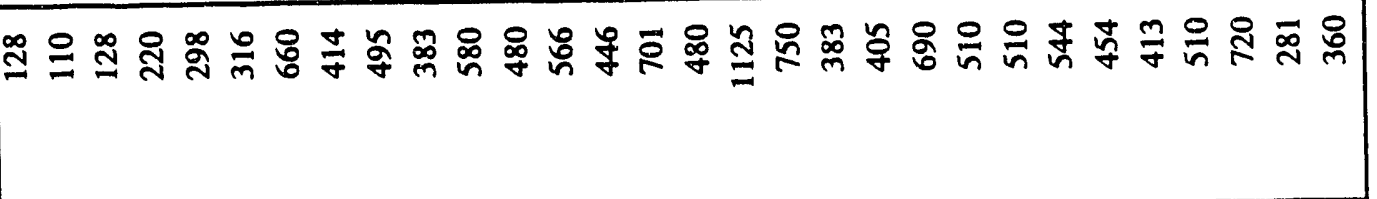 \\
\hline$\stackrel{8}{8}$ & 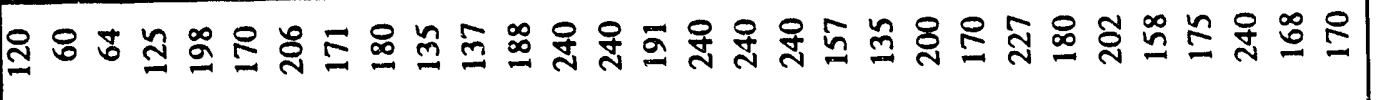 \\
\hline 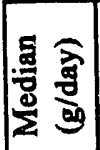 & అ \\
\hline$\ddot{n}$ & 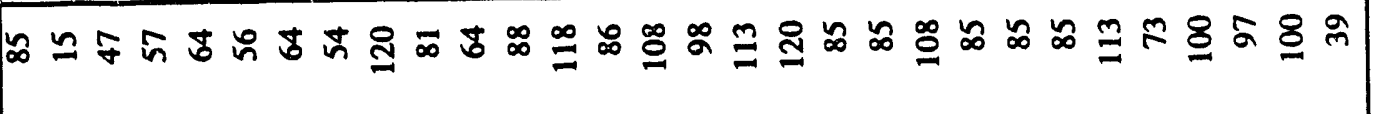 \\
\hline 量 & โ \\
\hline$\stackrel{\infty}{a}$ & 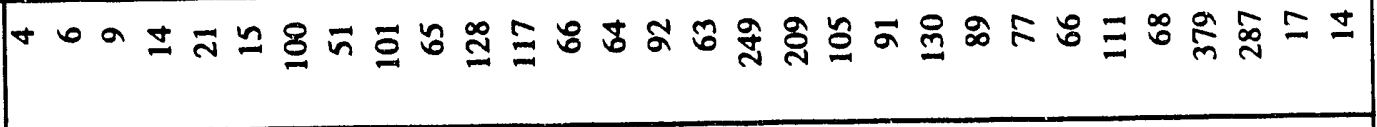 \\
\hline ن & 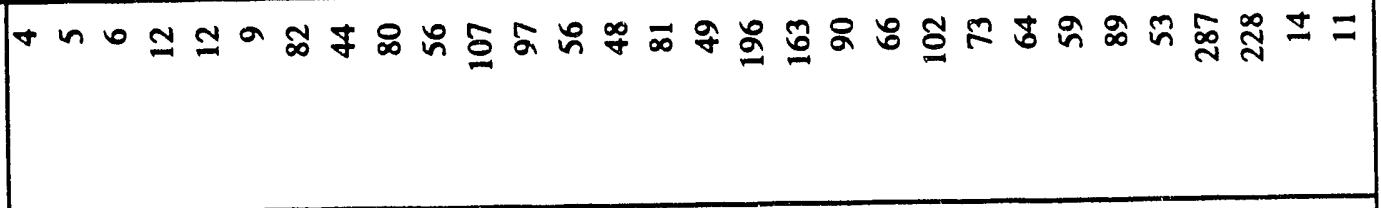 \\
\hline 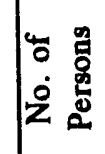 & 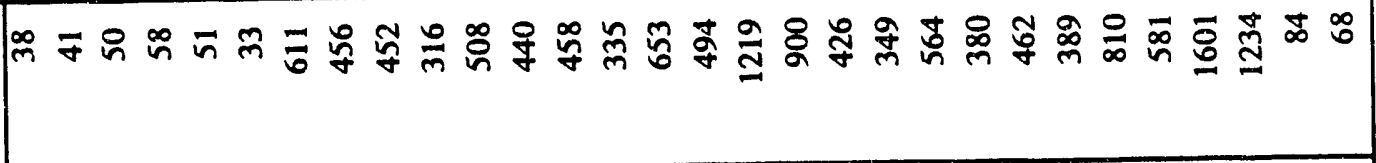 \\
\hline 苟 & 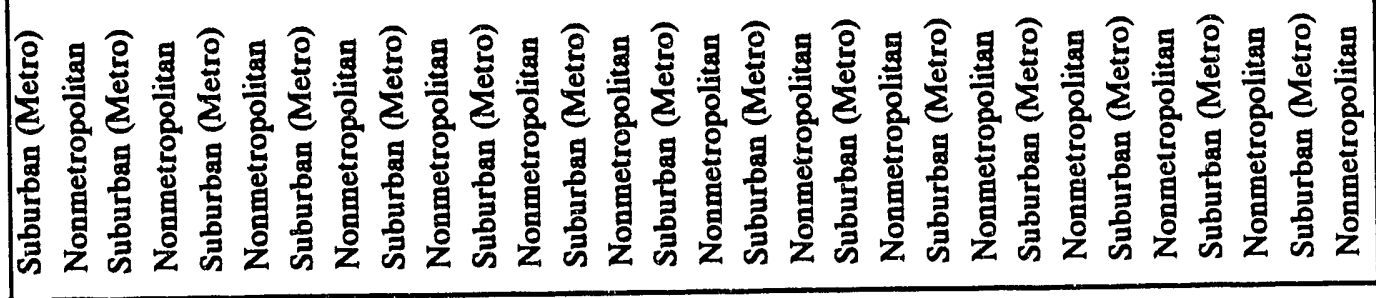 \\
\hline & 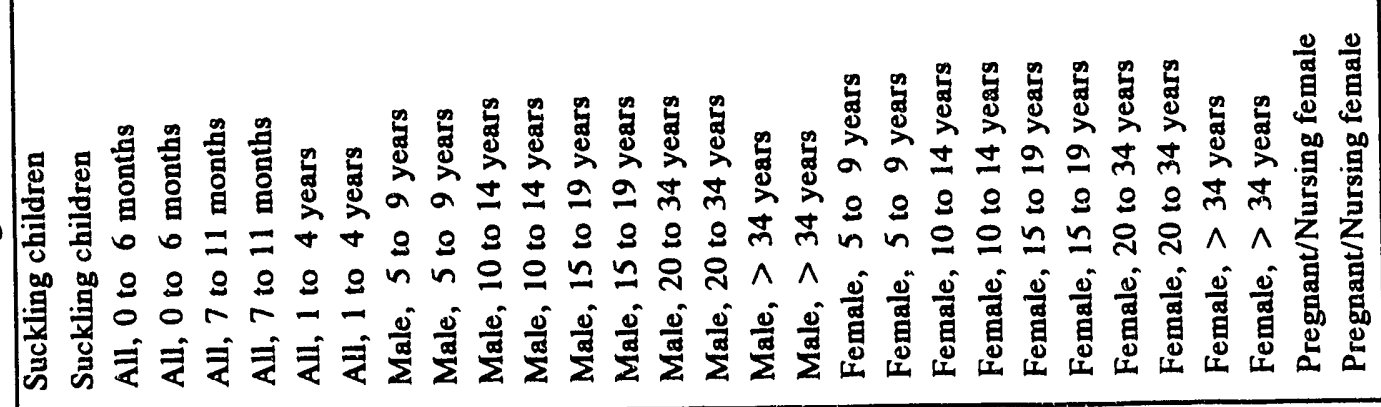 \\
\hline
\end{tabular}

C. 44 


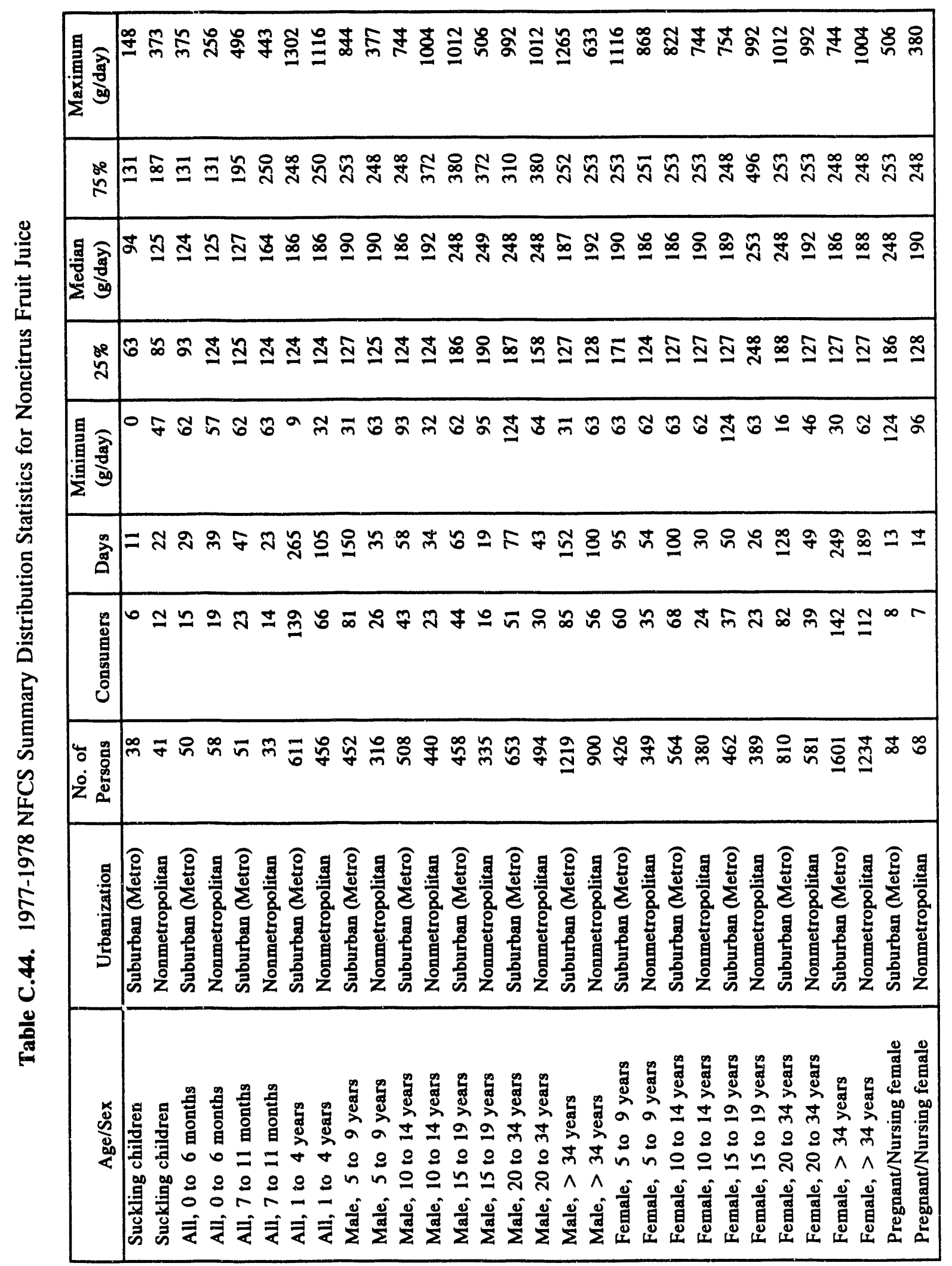




\begin{tabular}{|c|c|}
\hline 量 & 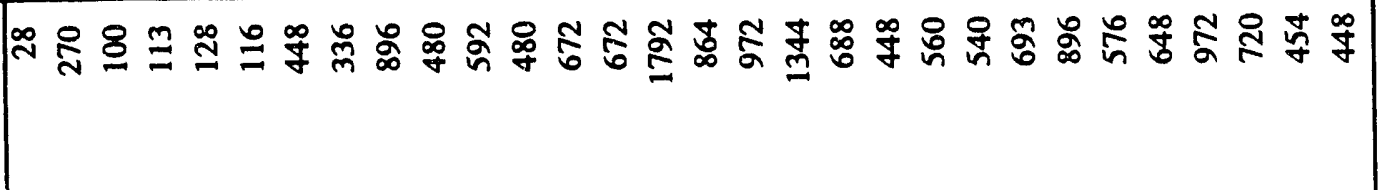 \\
\hline$\stackrel{8}{8}$ & 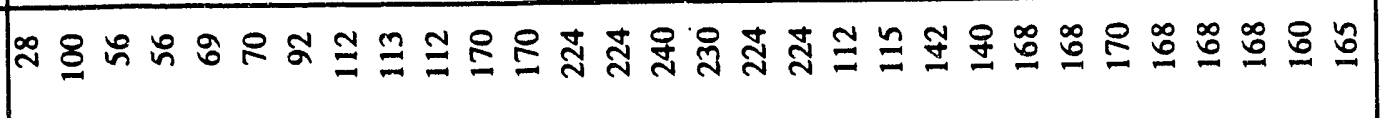 \\
\hline$\frac{3}{2}$ & 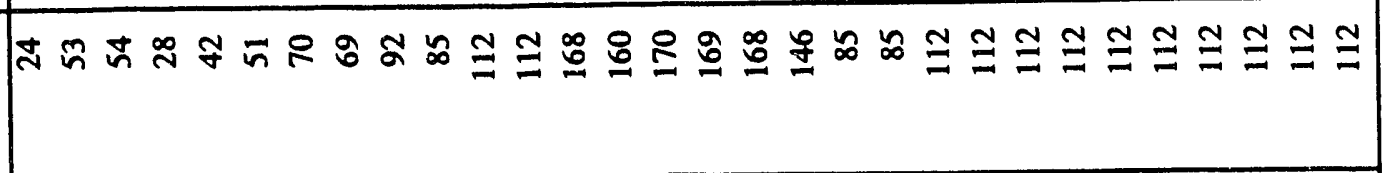 \\
\hline$\stackrel{3}{a}$ & 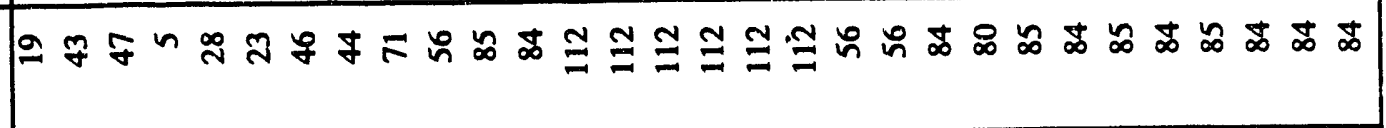 \\
\hline 量 & Ir- \\
\hline$\stackrel{\infty}{\Delta}$ & 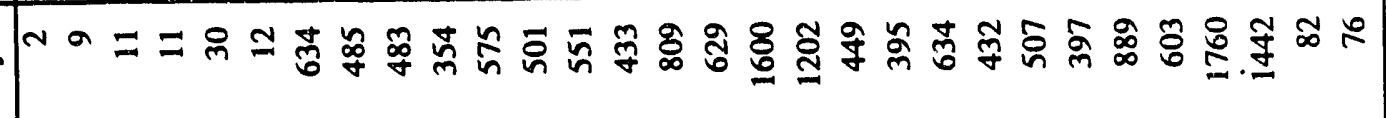 \\
\hline ن & 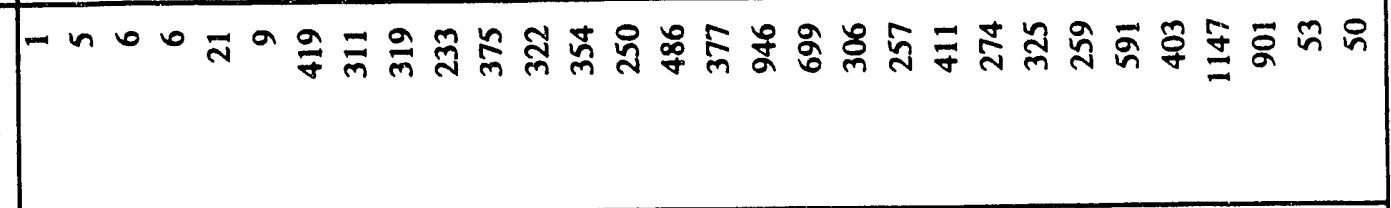 \\
\hline 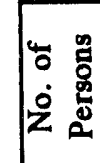 & 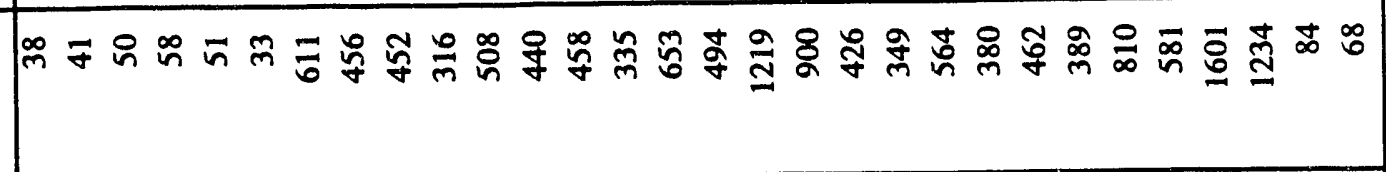 \\
\hline 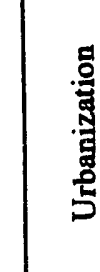 & 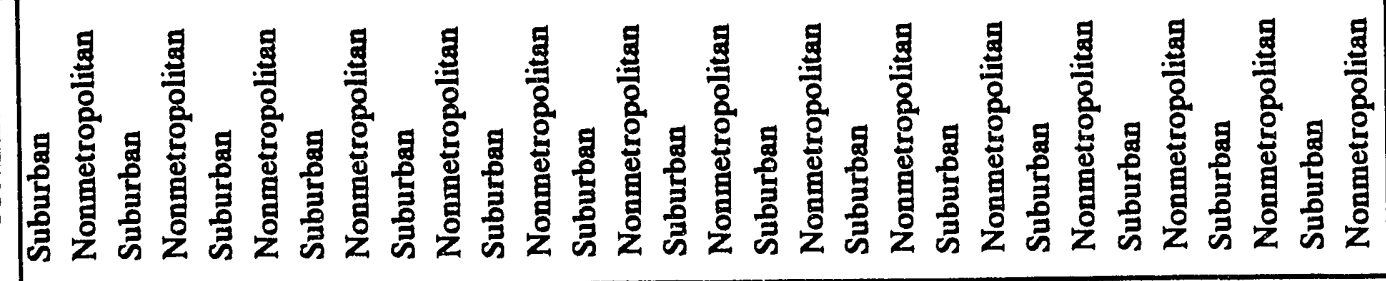 \\
\hline 然 & 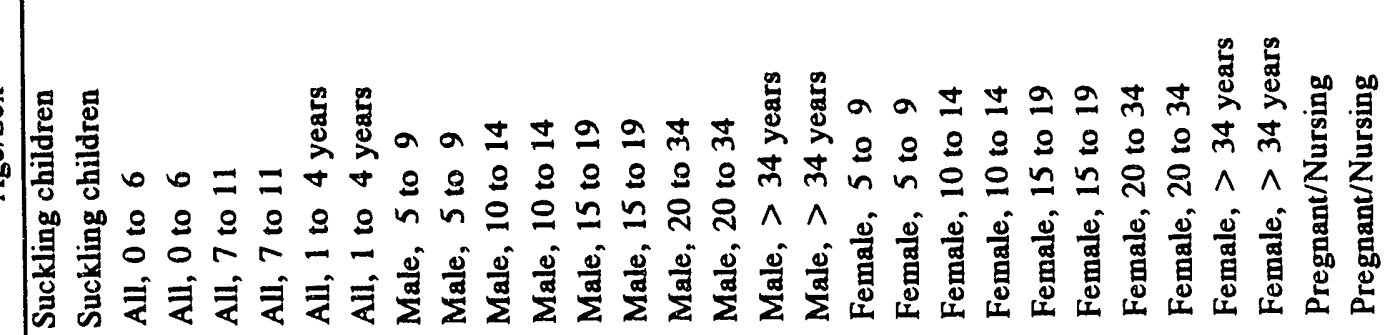 \\
\hline
\end{tabular}




\begin{tabular}{|c|c|}
\hline 要 & ळ \\
\hline$\stackrel{80}{\kappa}$ & D К \\
\hline 遱 & 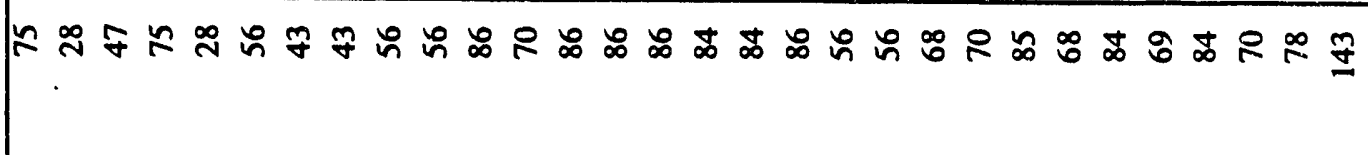 \\
\hline$\ddot{2}$ & 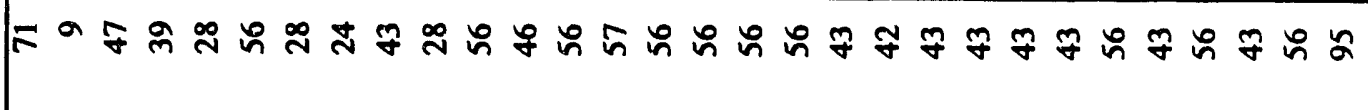 \\
\hline$\frac{1}{3}$ & 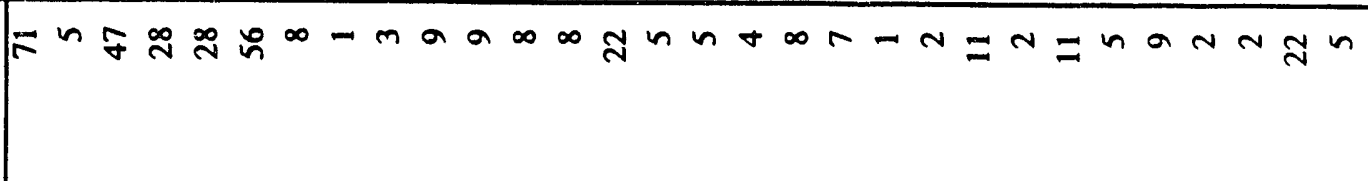 \\
\hline$\stackrel{\infty}{\mathrm{a}}$ & Nナm+m \\
\hline 要 & 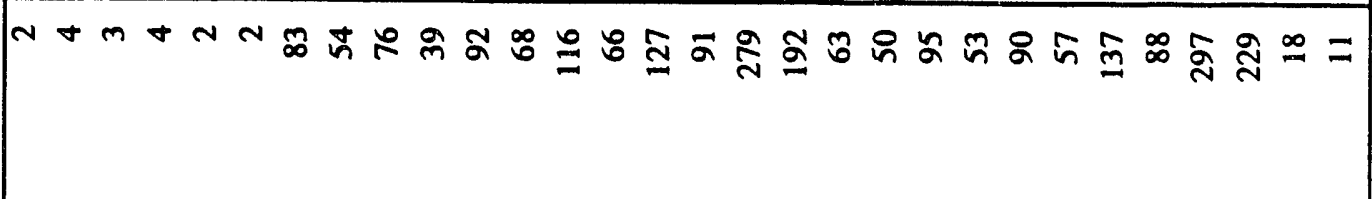 \\
\hline 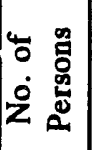 & 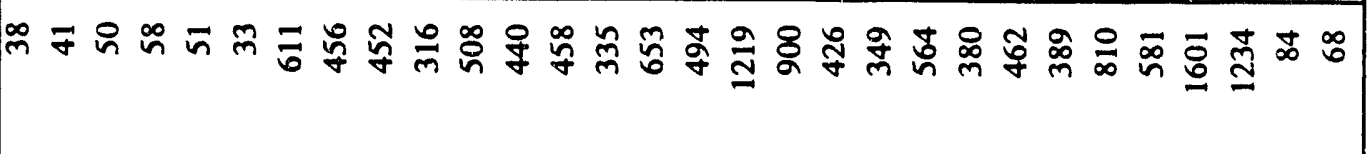 \\
\hline 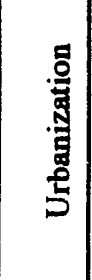 & 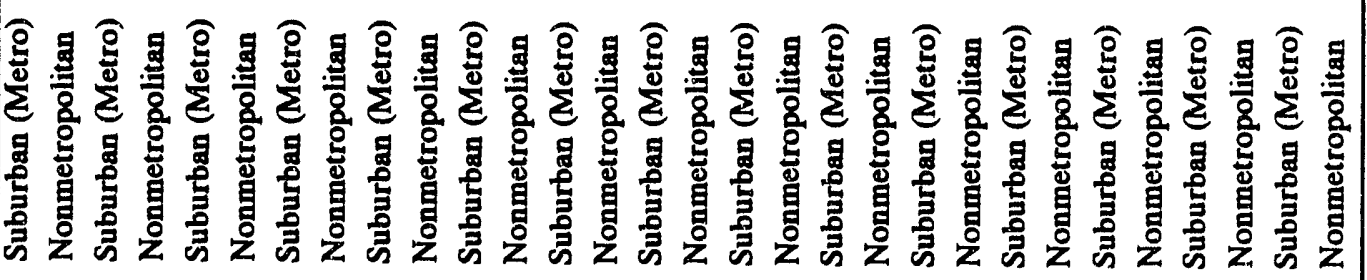 \\
\hline $\mid$ & 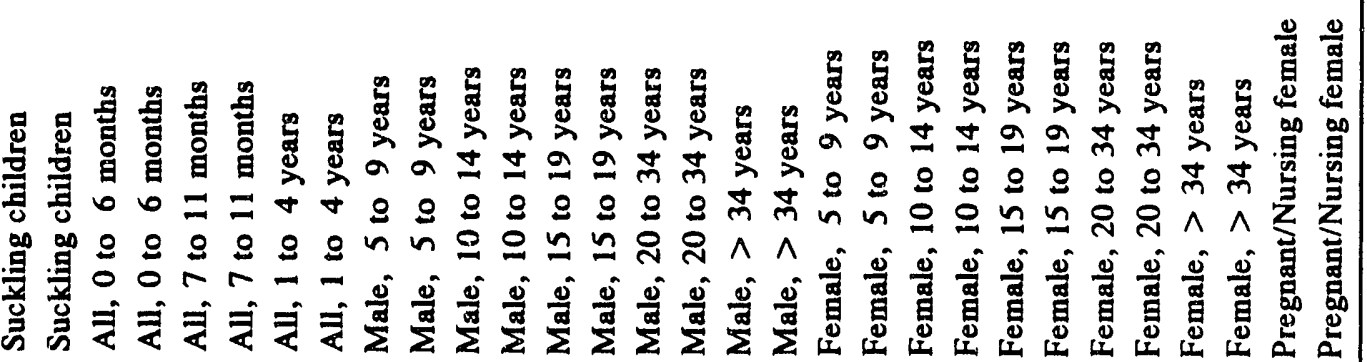 \\
\hline
\end{tabular}




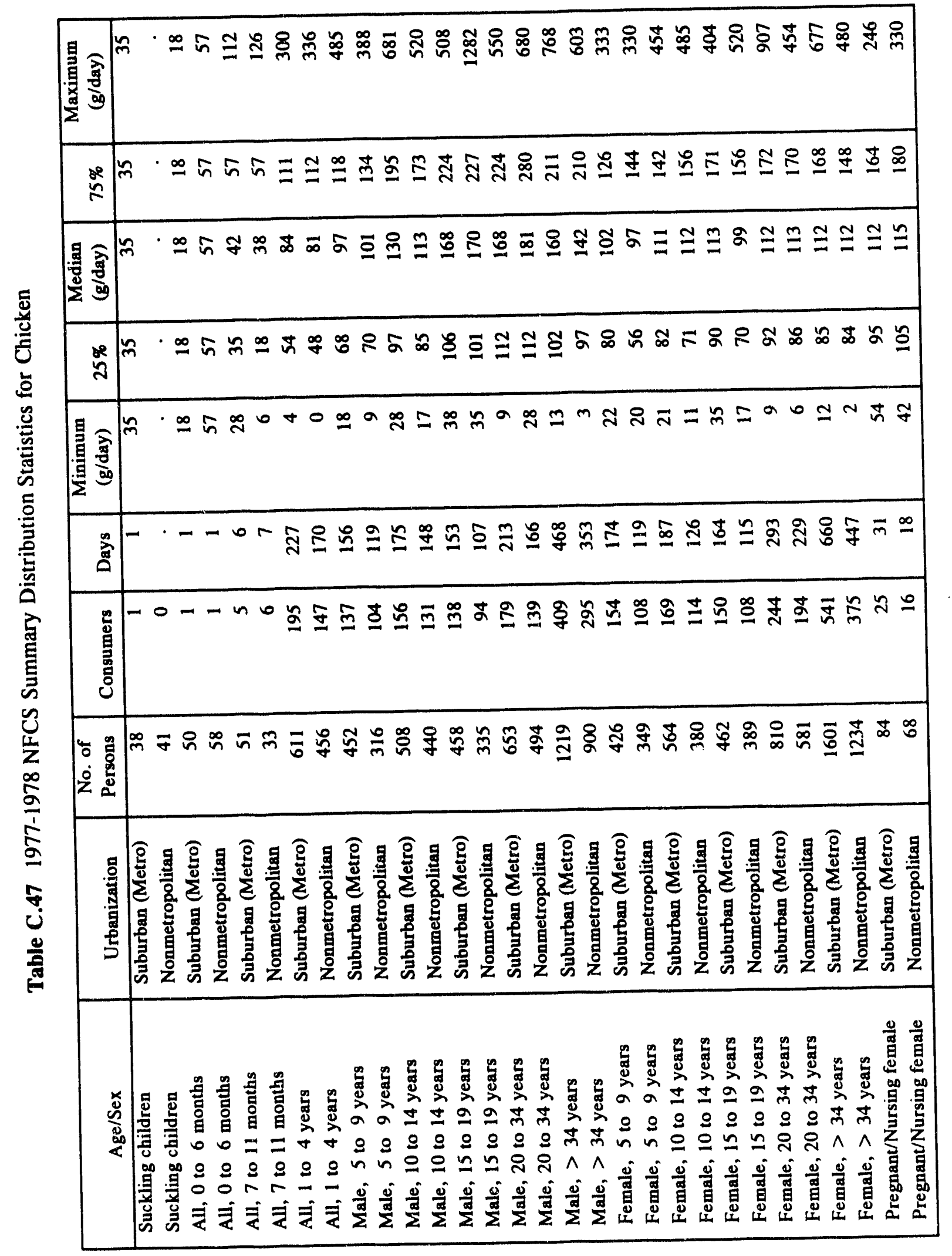

C. 48 


\begin{tabular}{|c|c|}
\hline 要 & 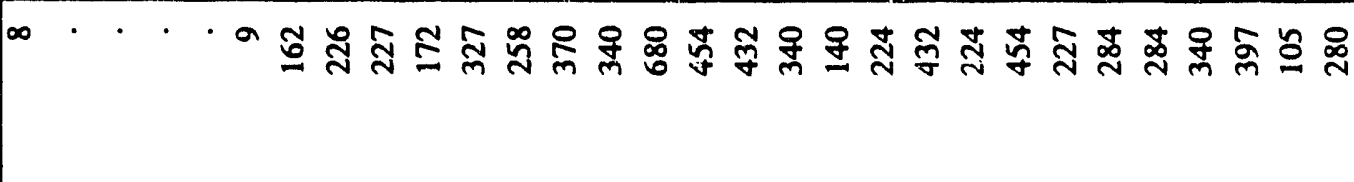 \\
\hline$\stackrel{8}{2}$ & 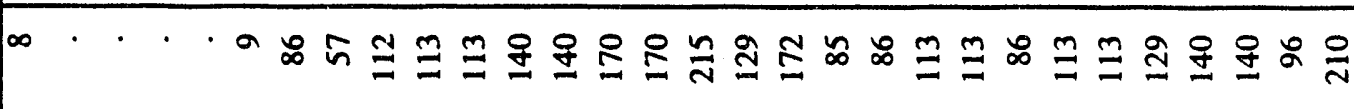 \\
\hline 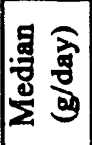 & 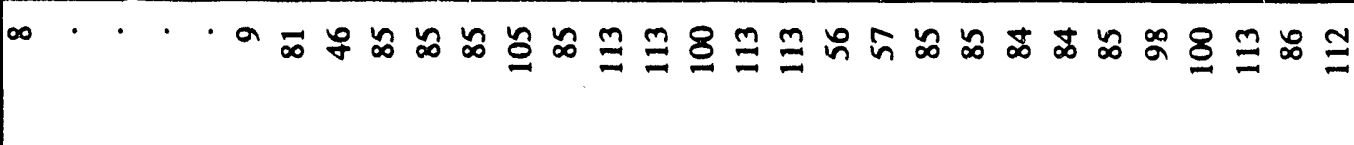 \\
\hline se & 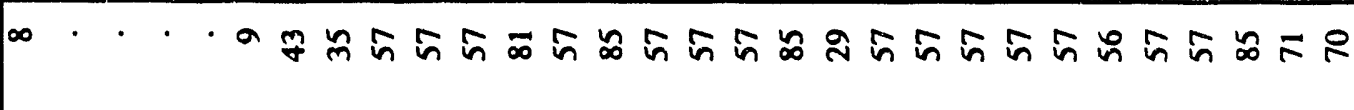 \\
\hline$\frac{1}{2}$ & 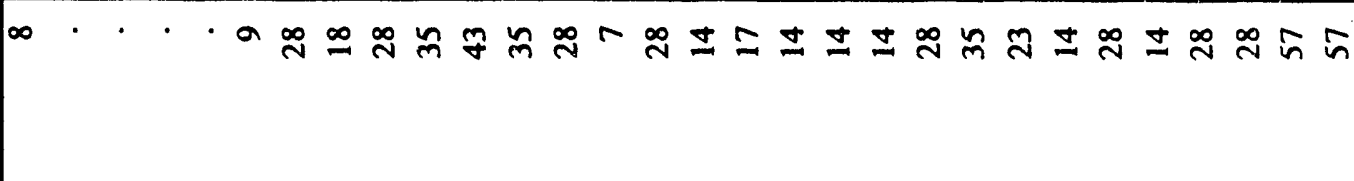 \\
\hline$\stackrel{\mathrm{m}}{\mathrm{I}}$ & 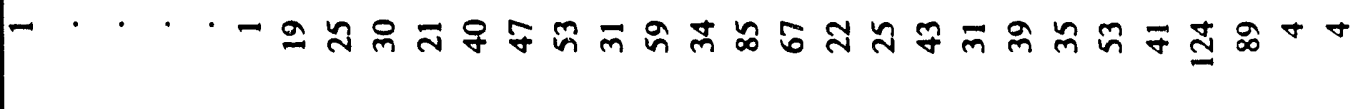 \\
\hline 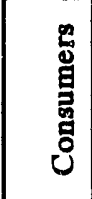 & - \\
\hline 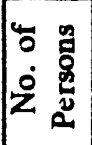 & 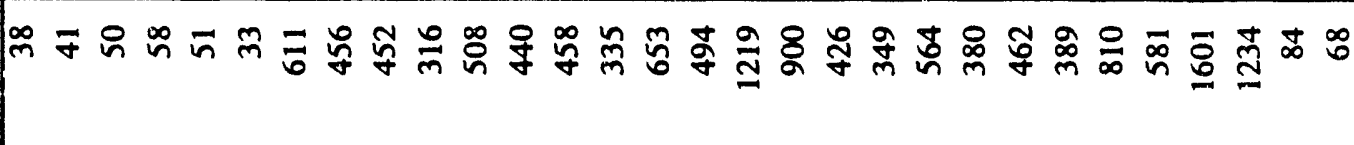 \\
\hline 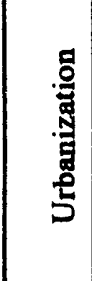 & 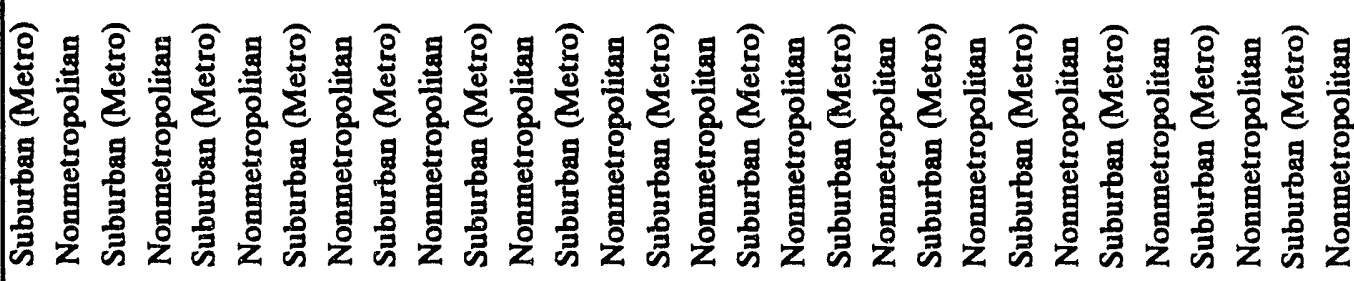 \\
\hline 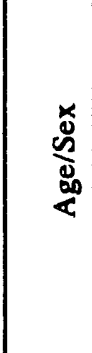 & 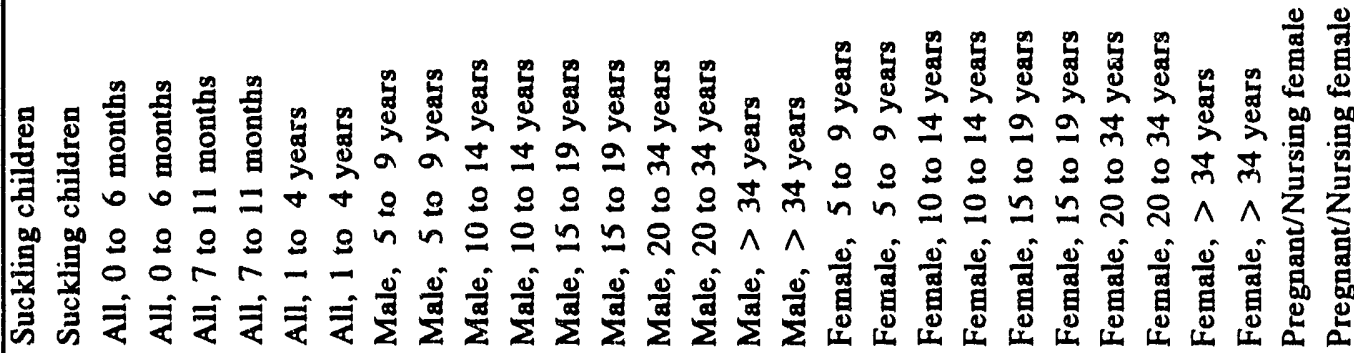 \\
\hline
\end{tabular}




\begin{tabular}{|c|c|}
\hline 量 & 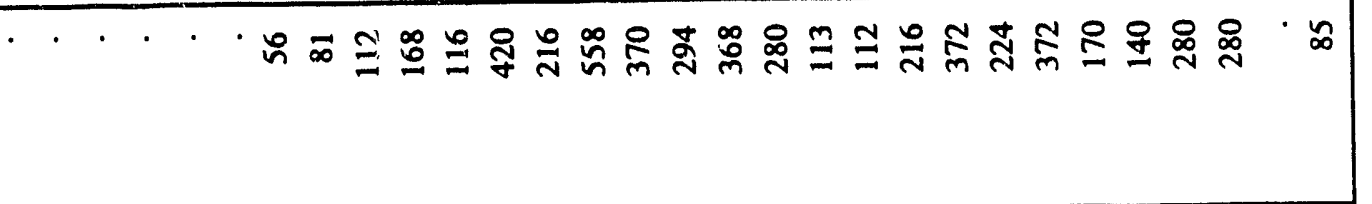 \\
\hline 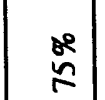 & 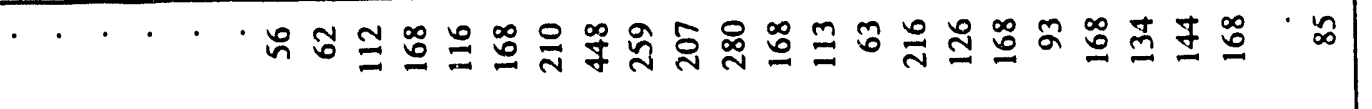 \\
\hline $\mid$ & 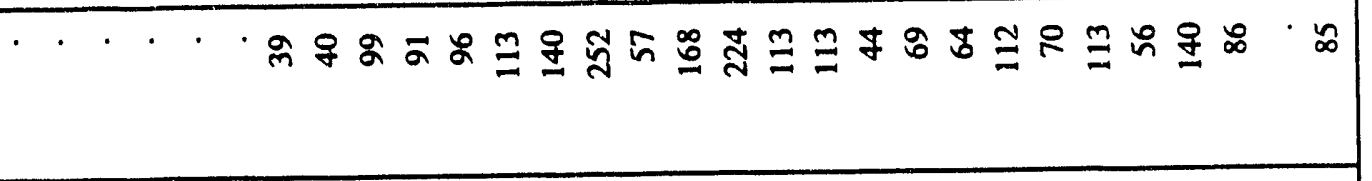 \\
\hline$\ddot{z}$ & 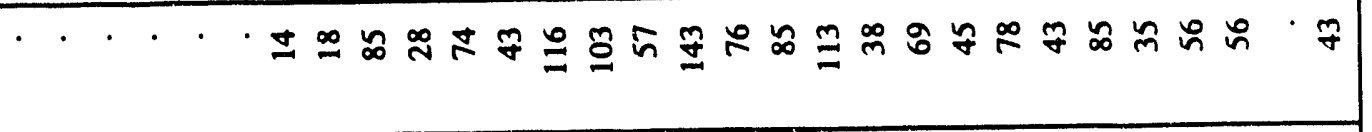 \\
\hline$\frac{1}{2}$ & 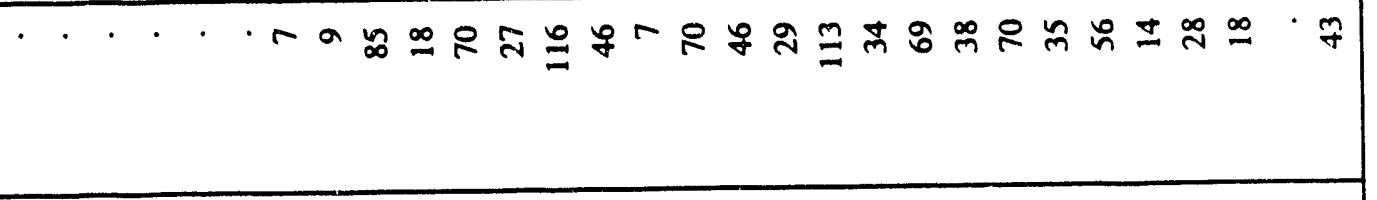 \\
\hline$\stackrel{\infty}{a}$ & 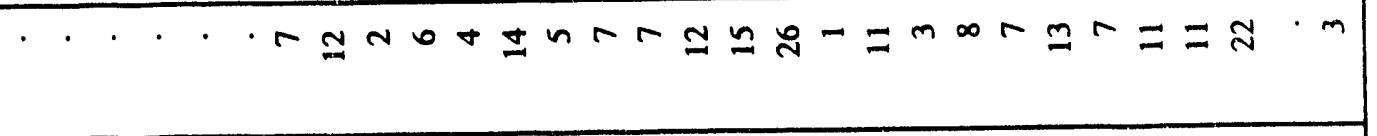 \\
\hline $\begin{array}{l}0 \\
0 \\
0 \\
0 \\
0 \\
0\end{array}$ & 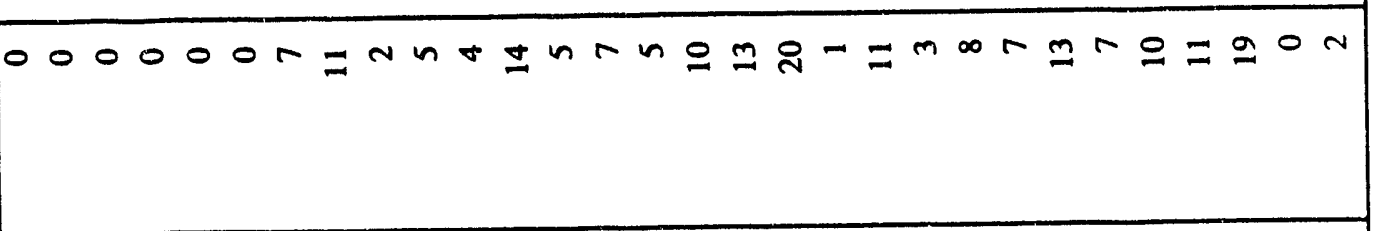 \\
\hline 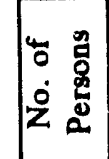 & 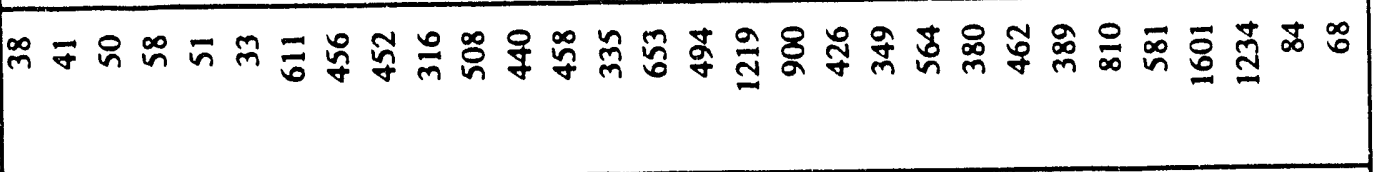 \\
\hline 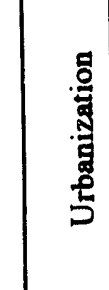 & 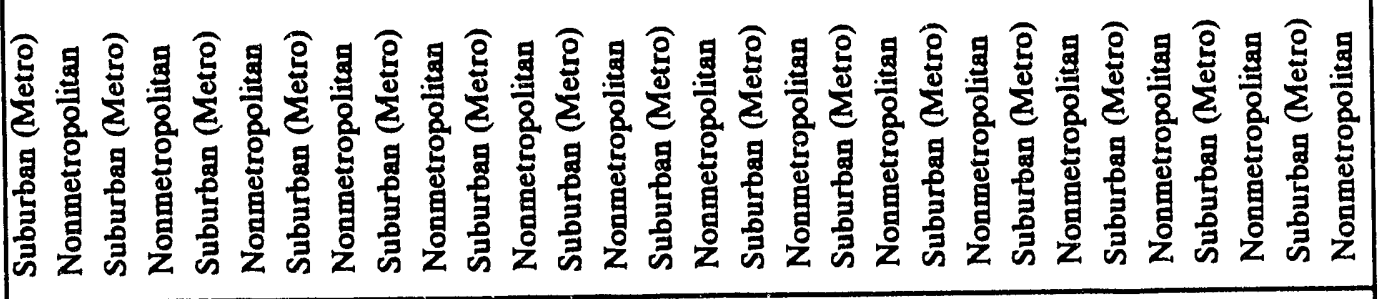 \\
\hline רִ & 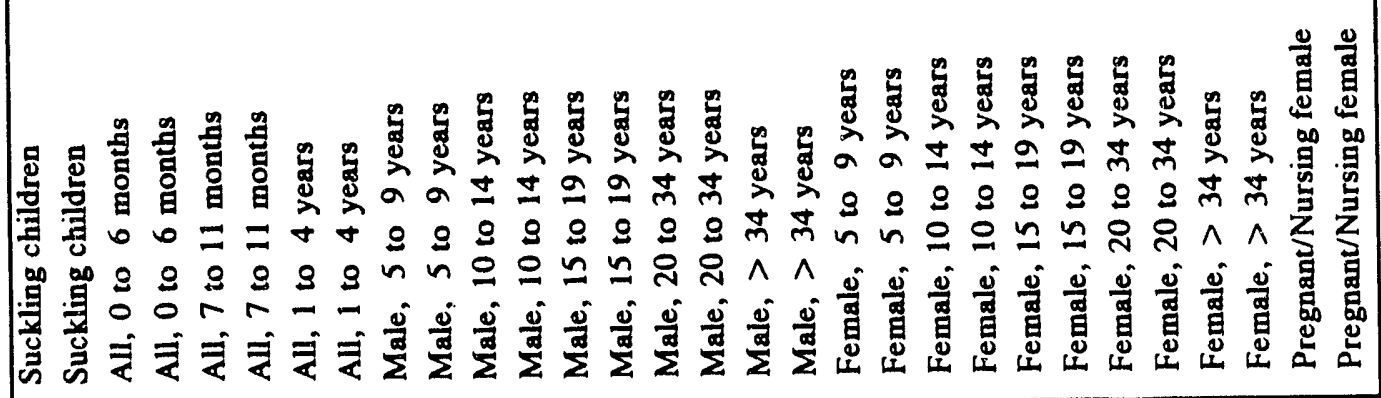 \\
\hline
\end{tabular}




\begin{tabular}{|c|c|c|}
\hline 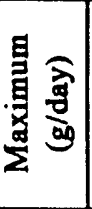 & $\oiiint \infty \cdot \cdots$ & 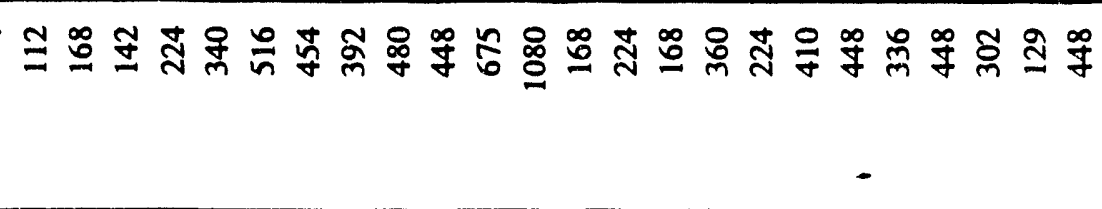 \\
\hline$\stackrel{s}{n}$ & 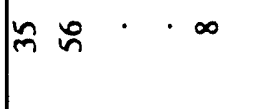 & 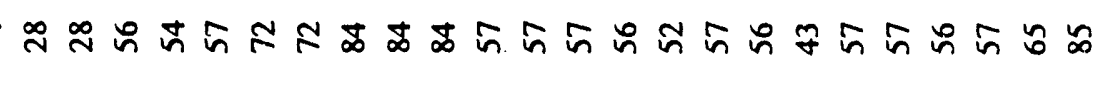 \\
\hline 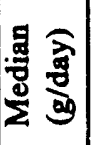 & $\sim \infty$ & 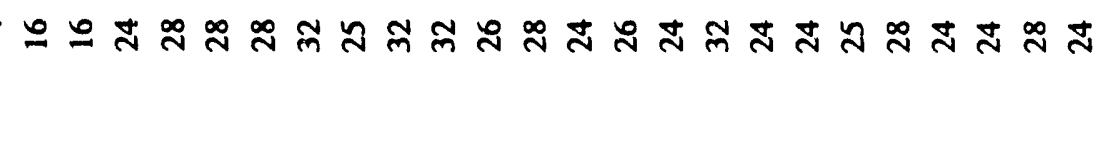 \\
\hline$\ddot{y}$ & $\cong \simeq \cdot \infty$ & 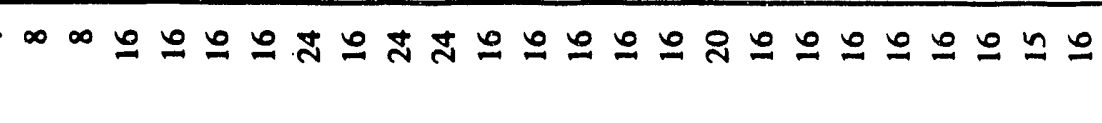 \\
\hline$\frac{1}{\frac{1}{3}}$ & $\cong \cong \cdot \infty$ & 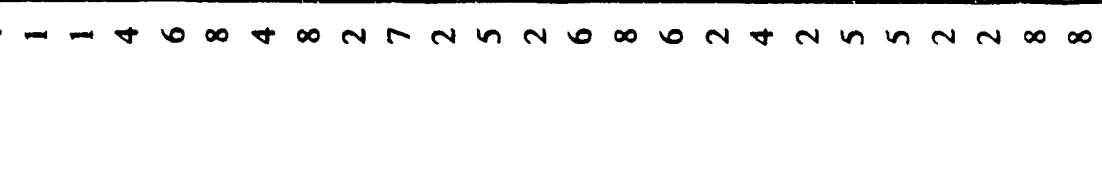 \\
\hline$\stackrel{\infty}{\vec{\Delta}}$ & 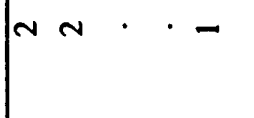 & 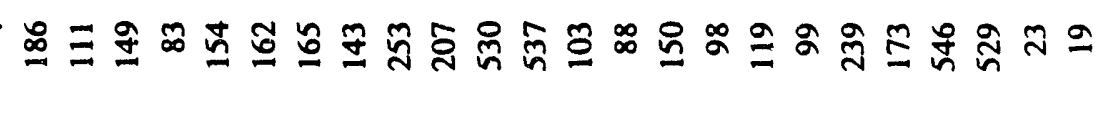 \\
\hline 惫 & 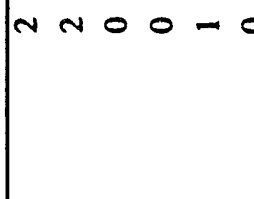 & 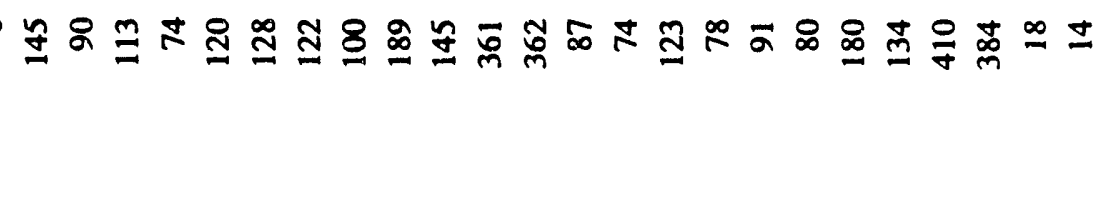 \\
\hline 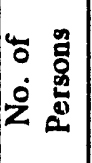 & 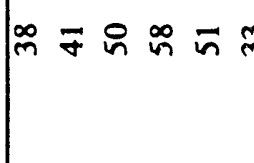 & 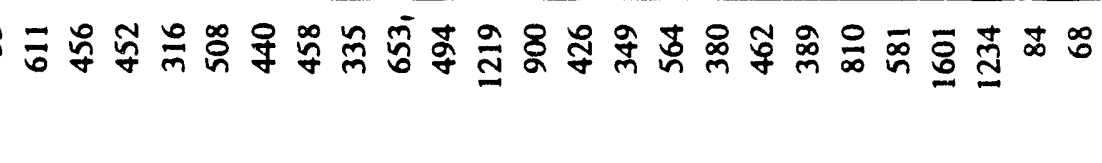 \\
\hline : & 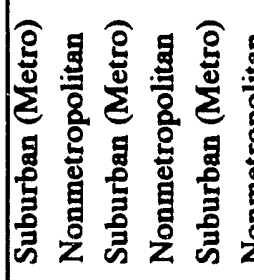 & 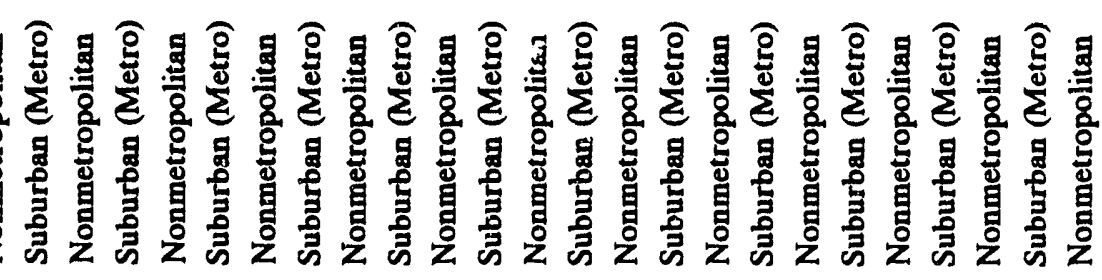 \\
\hline \multicolumn{3}{|c|}{ 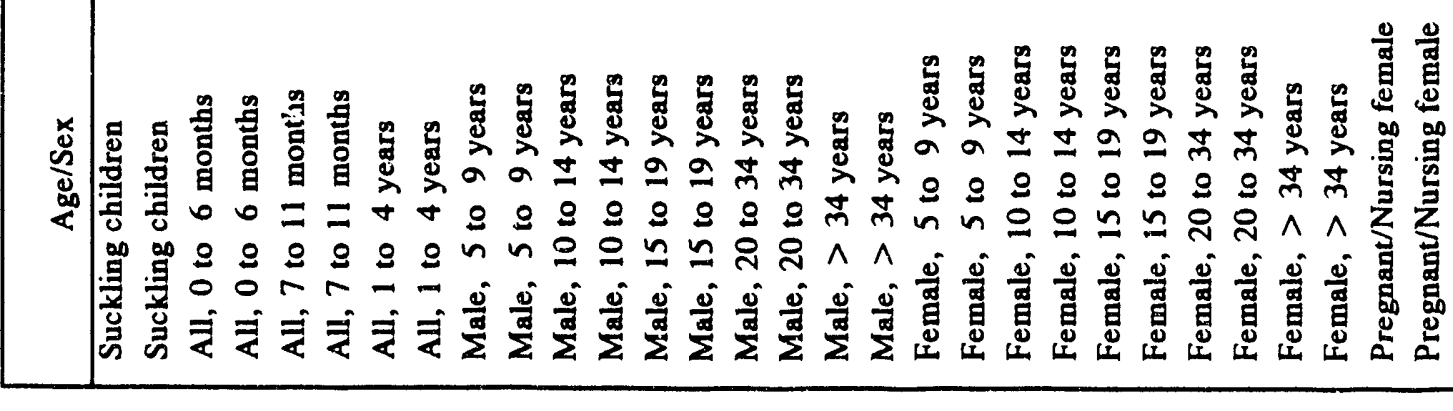 } \\
\hline
\end{tabular}




\begin{tabular}{|c|c|}
\hline 量拿 & 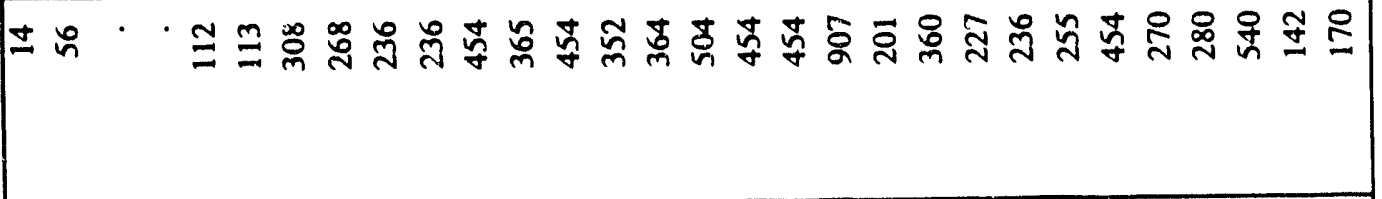 \\
\hline$\stackrel{8}{\approx}$ & 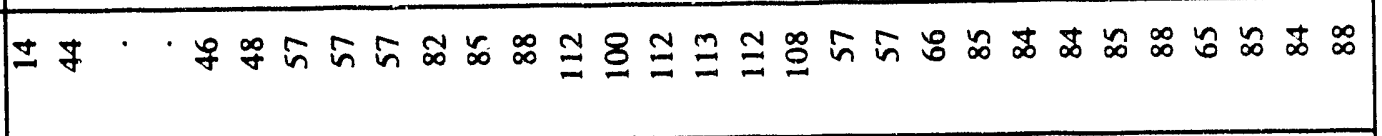 \\
\hline 递要 & 士心 \\
\hline$y$ & 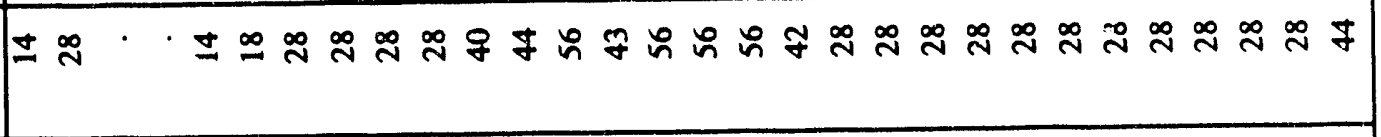 \\
\hline 䕗 & In. \\
\hline$\stackrel{n}{a}$ & 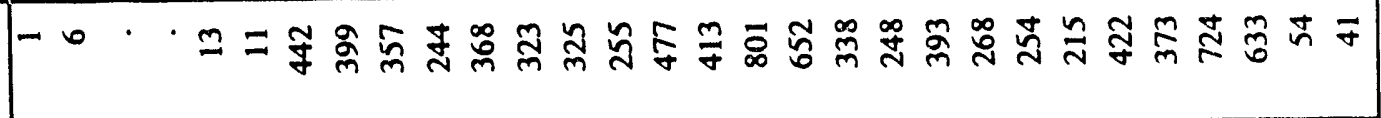 \\
\hline 兽 & - \\
\hline 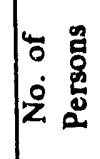 & 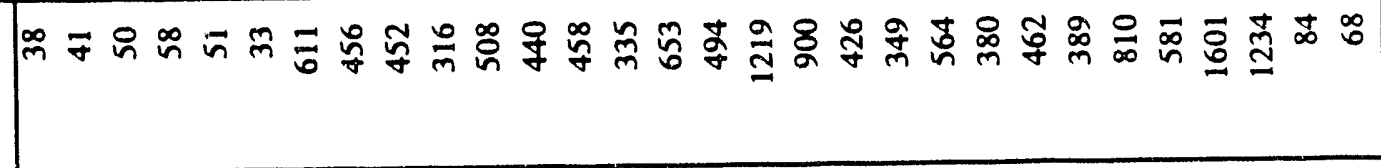 \\
\hline 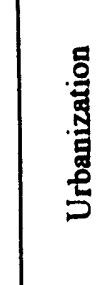 & 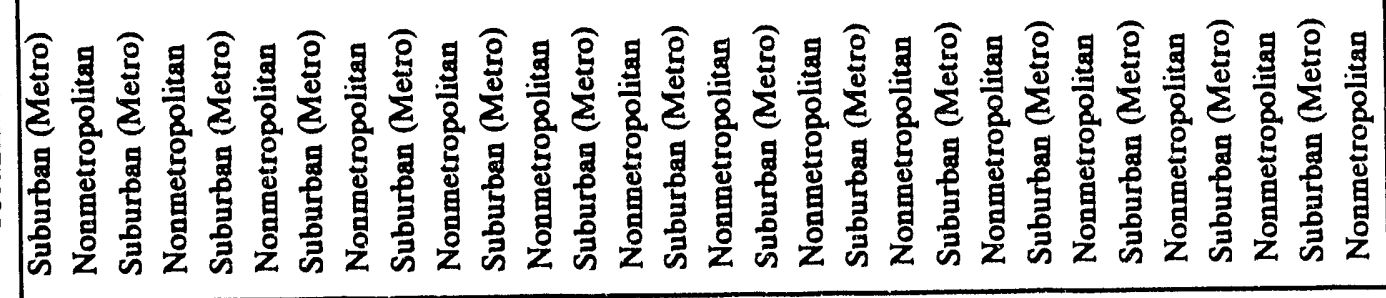 \\
\hline < & 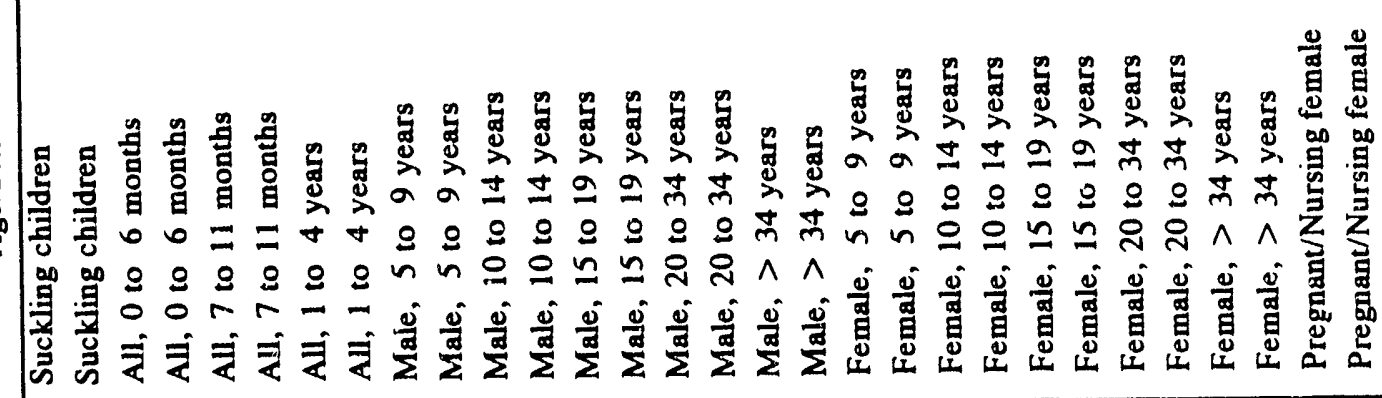 \\
\hline
\end{tabular}

C. 52 


\begin{tabular}{|c|c|}
\hline 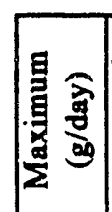 & 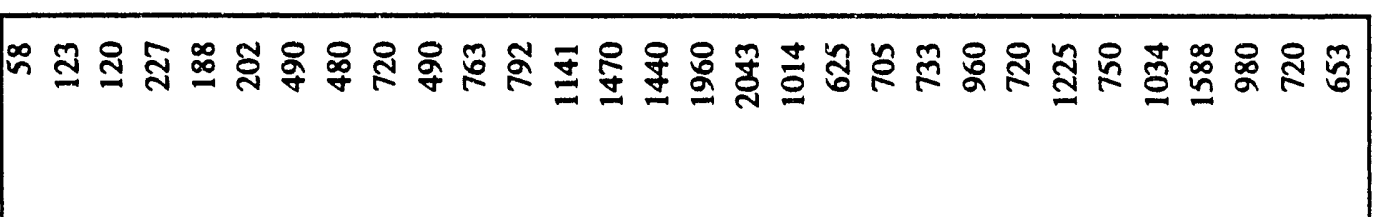 \\
\hline \& & 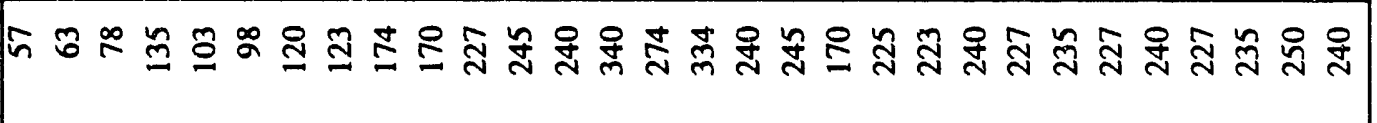 \\
\hline 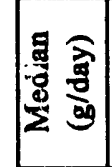 & 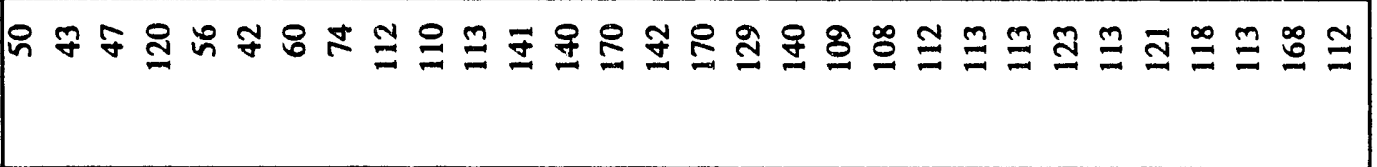 \\
\hline is & 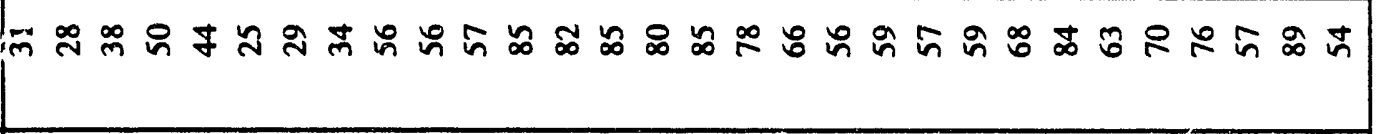 \\
\hline 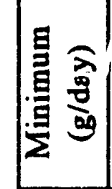 & 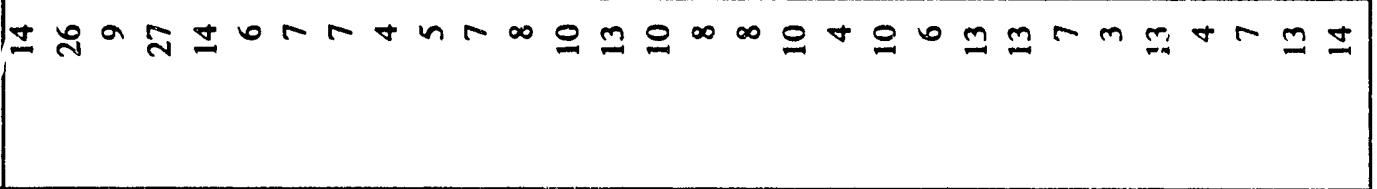 \\
\hline$\stackrel{m}{a}$ & 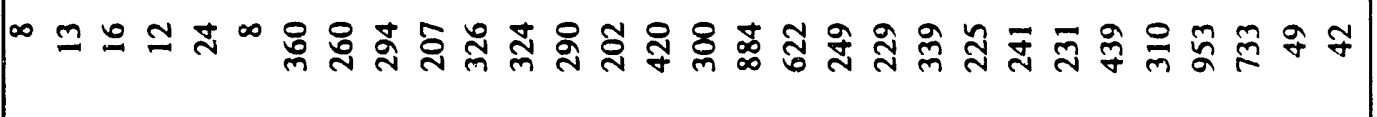 \\
\hline 童 & | \\
\hline 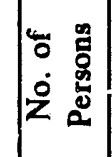 & 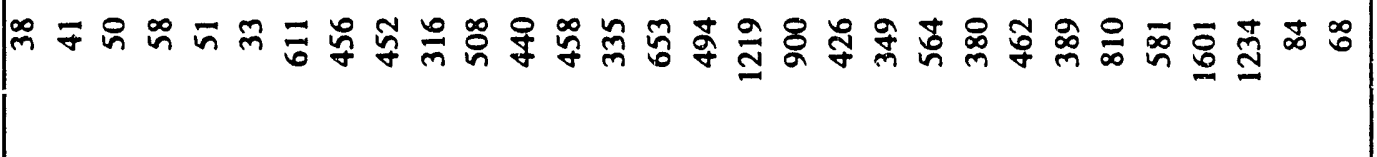 \\
\hline & 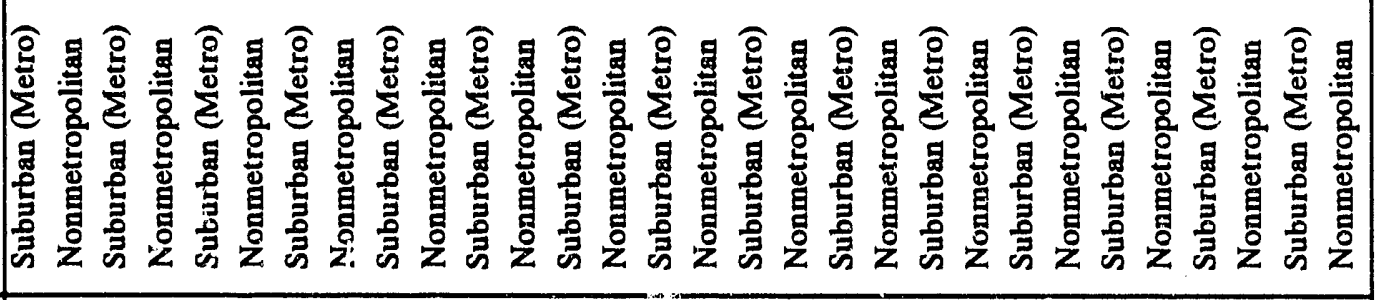 \\
\hline & 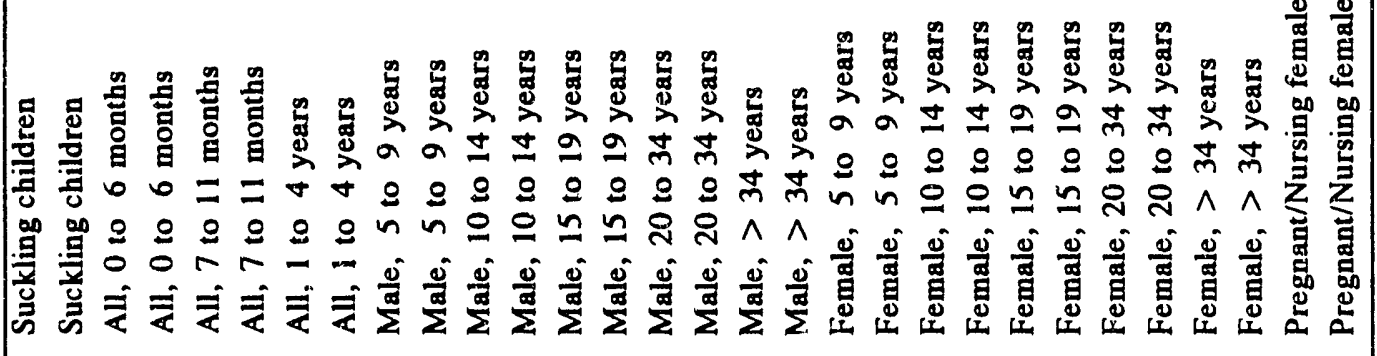 \\
\hline
\end{tabular}




\begin{tabular}{|c|c|}
\hline 量拿 & 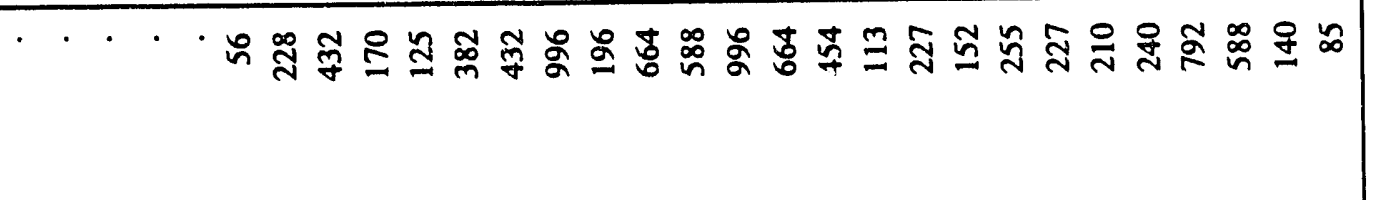 \\
\hline : & 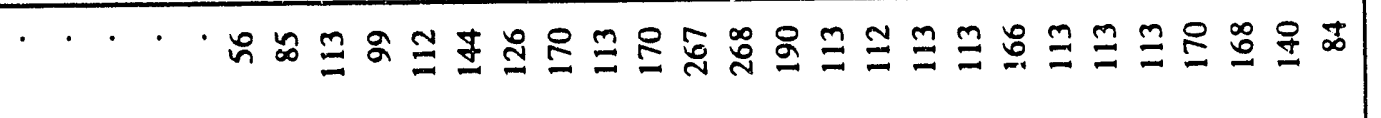 \\
\hline $\mid$ & 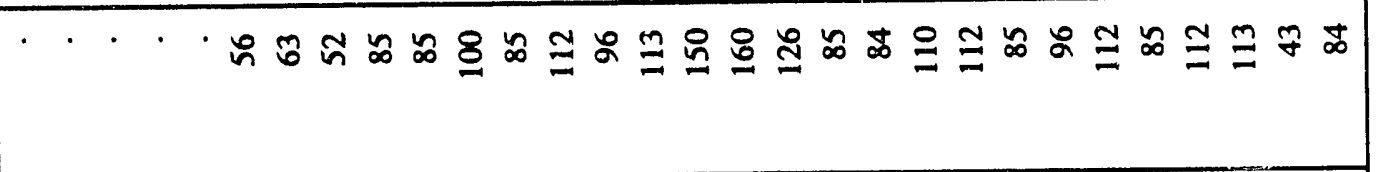 \\
\hline a & 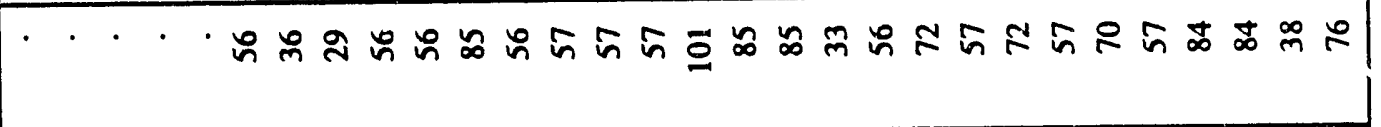 \\
\hline 量 & 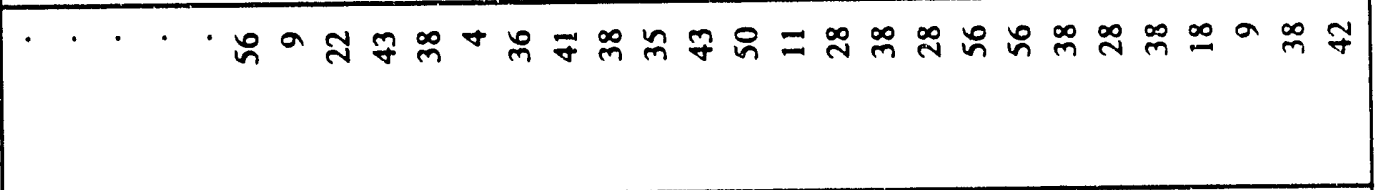 \\
\hline$\stackrel{\infty}{\mathrm{g}}$ & 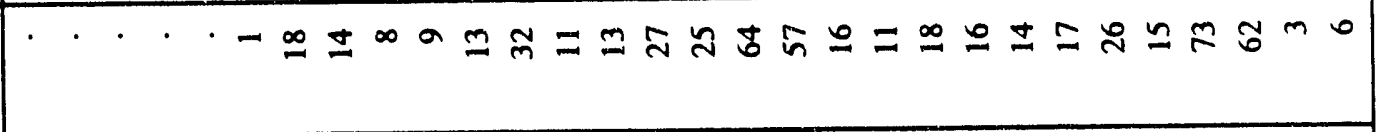 \\
\hline 竞 & 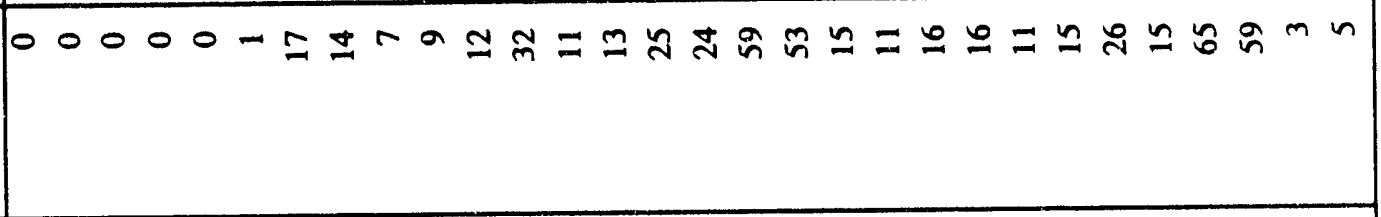 \\
\hline 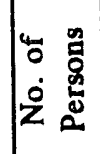 & 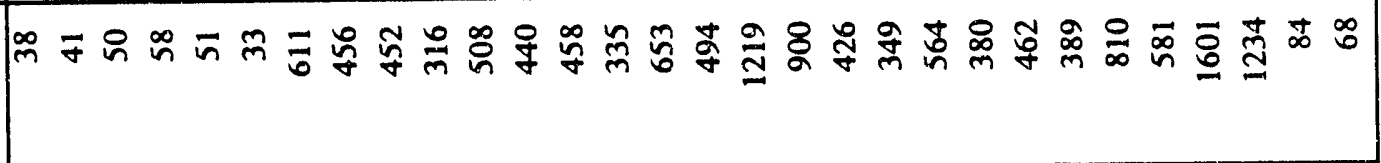 \\
\hline 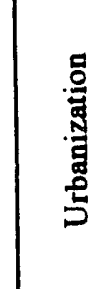 & 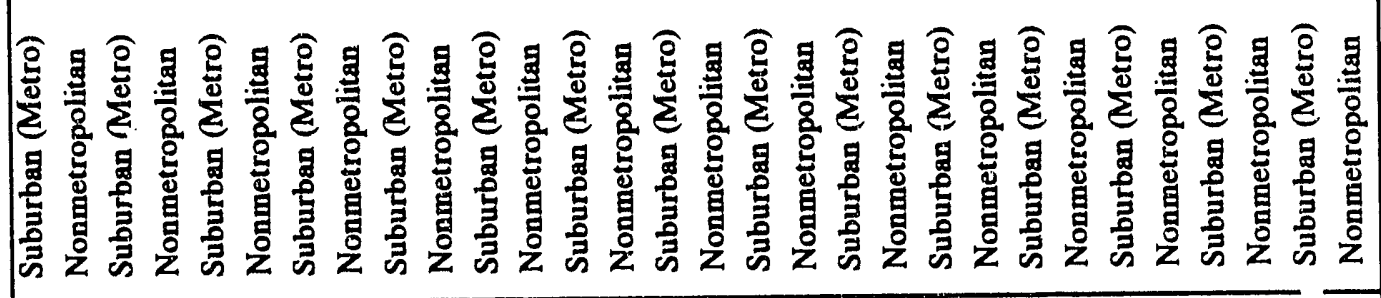 \\
\hline & 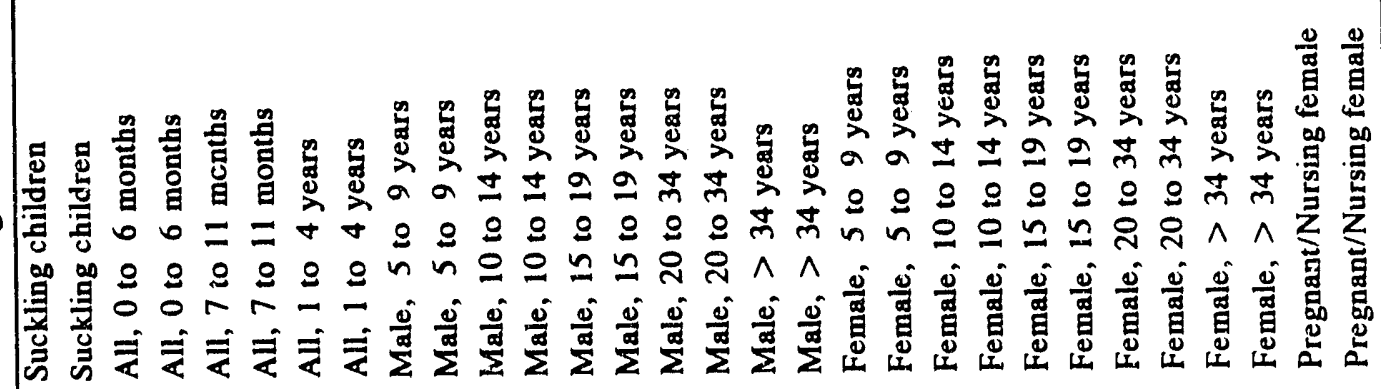 \\
\hline
\end{tabular}




\begin{tabular}{|c|c|}
\hline$\frac{1}{2}$ & 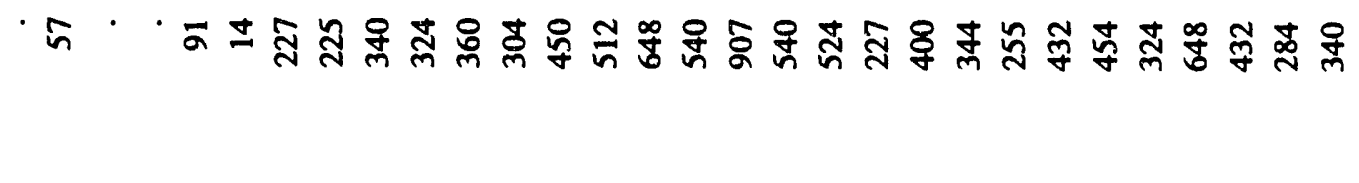 \\
\hline$\stackrel{8}{2}$ & 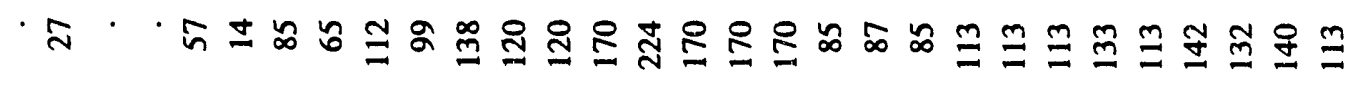 \\
\hline$\frac{0}{\frac{2}{2}}$ & 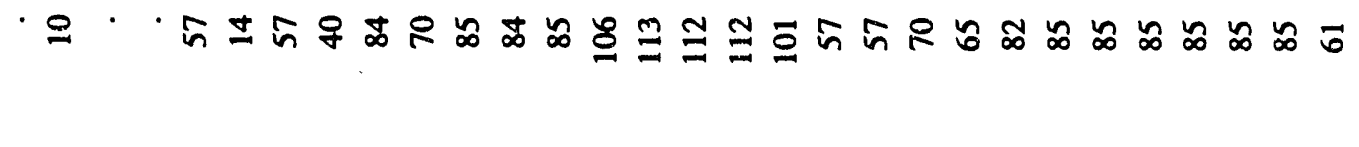 \\
\hline$\stackrel{y}{20}$ & 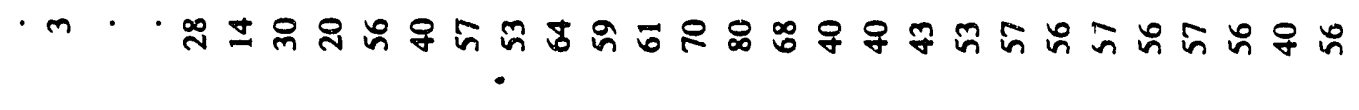 \\
\hline$\frac{1}{2}$ & 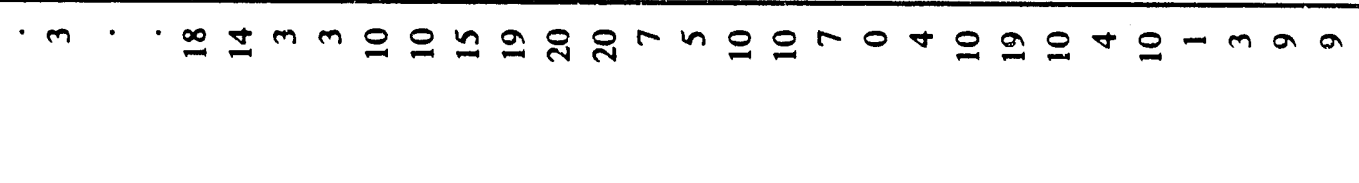 \\
\hline$\stackrel{n}{n}$ & 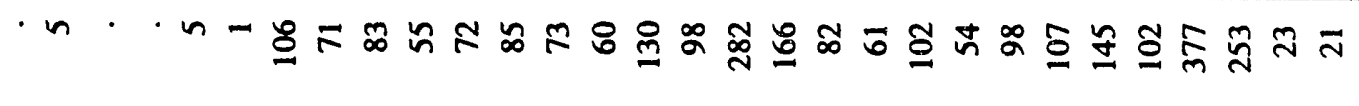 \\
\hline 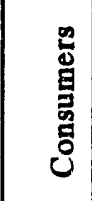 & 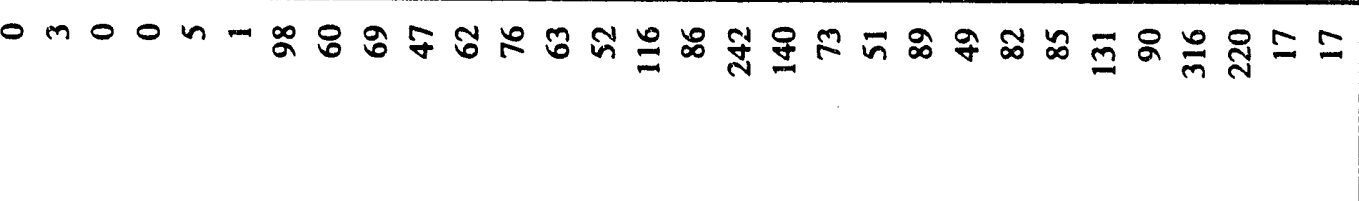 \\
\hline 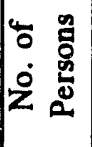 & 冓 \\
\hline 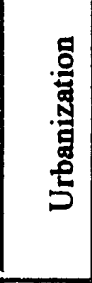 & 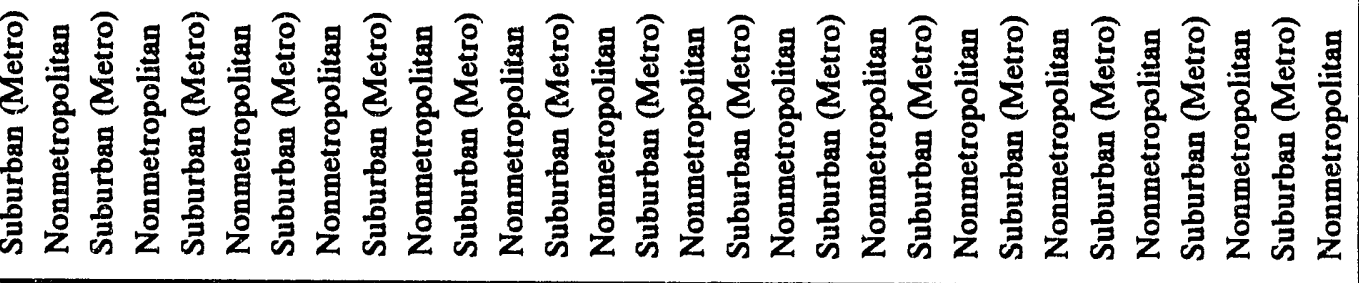 \\
\hline 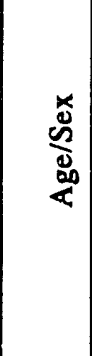 & 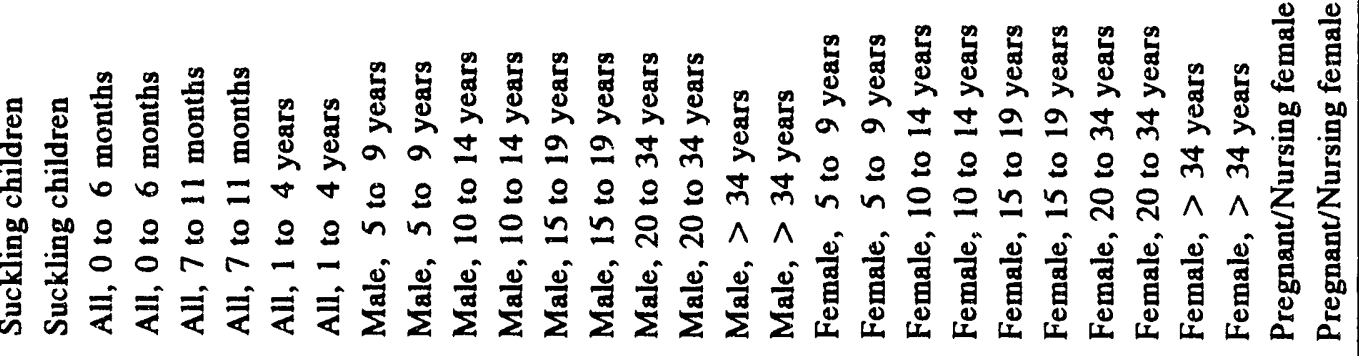 \\
\hline
\end{tabular}




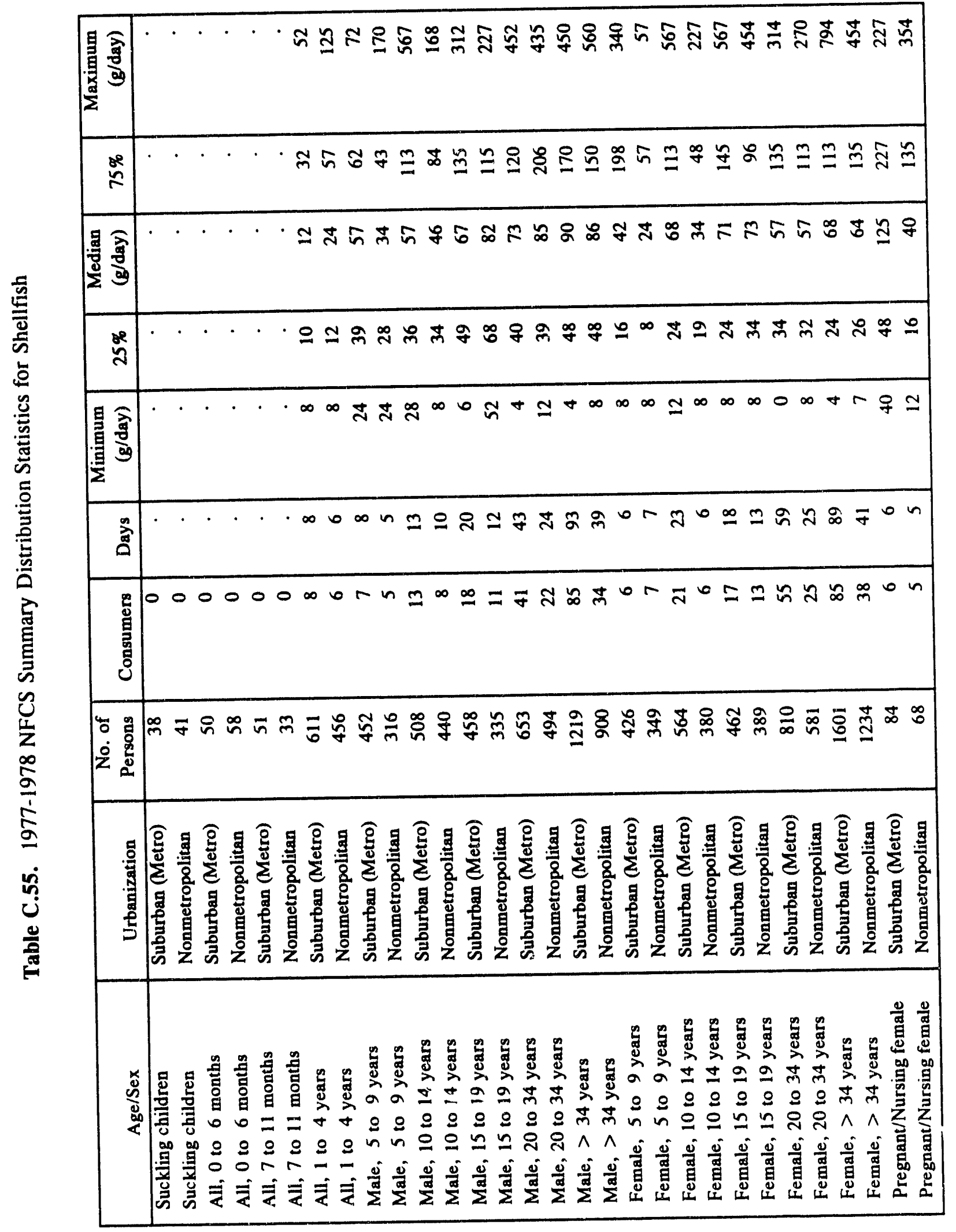

C. 56 


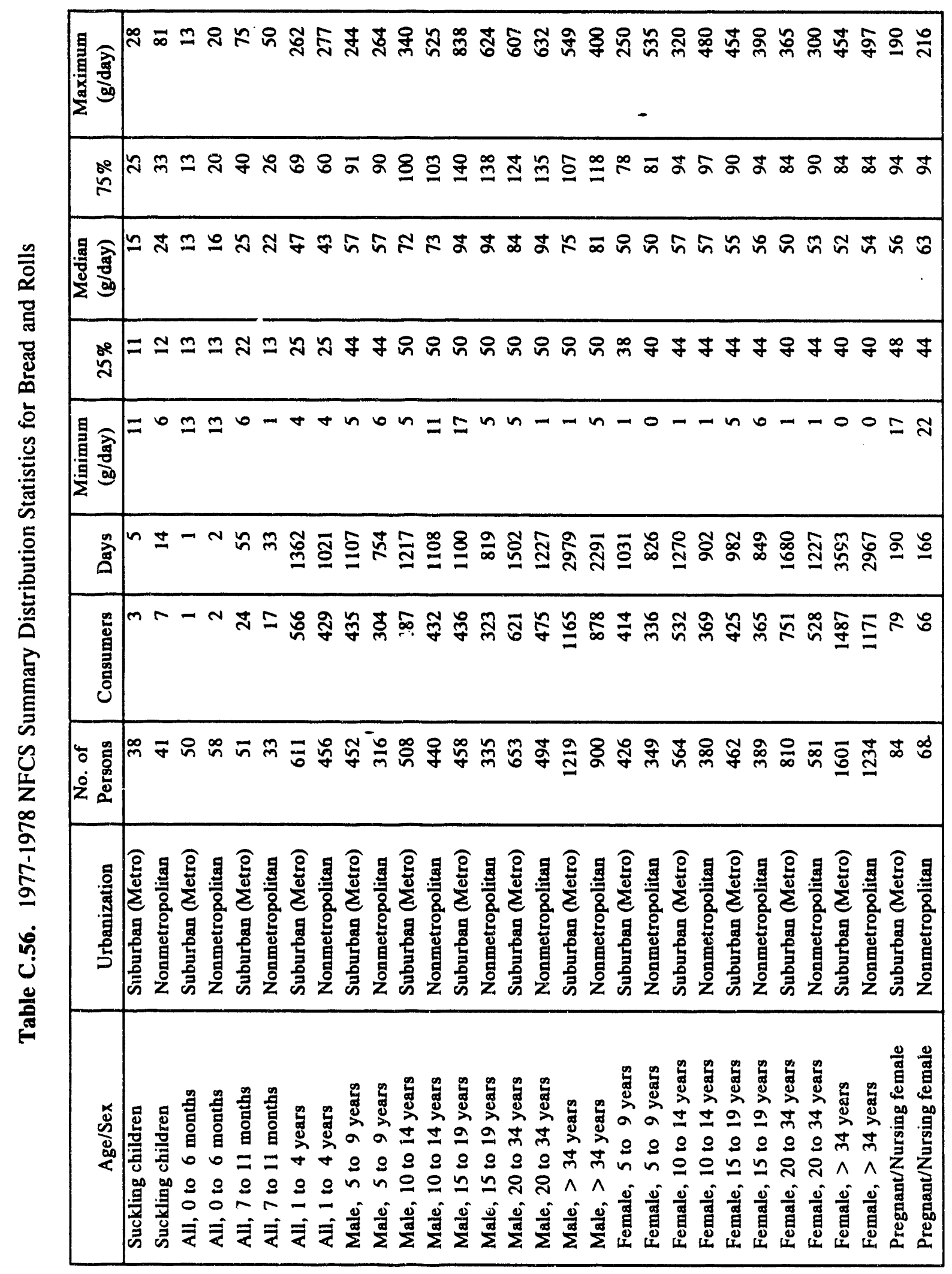

C. 57 


\begin{tabular}{|c|c|}
\hline 要 & 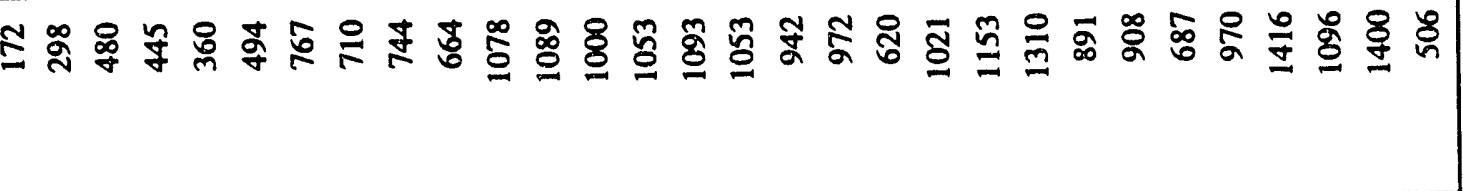 \\
\hline$\therefore$ & 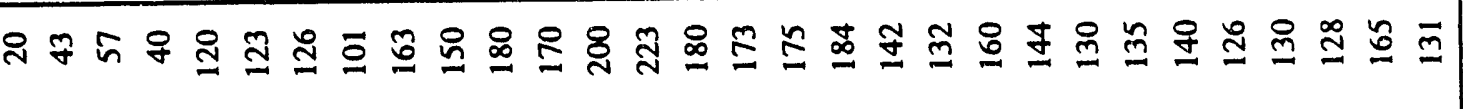 \\
\hline 焉 & $=$ ล \\
\hline$y$ & 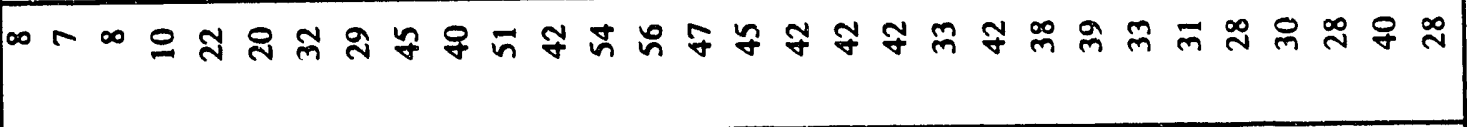 \\
\hline 量新 & 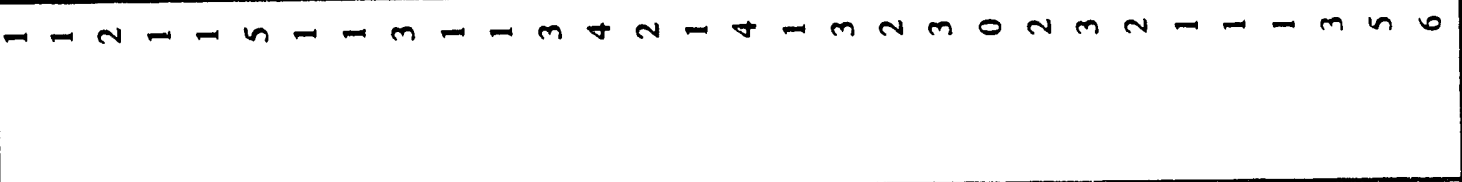 \\
\hline 鿖 & 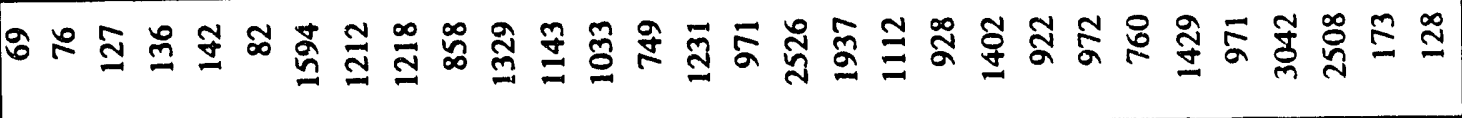 \\
\hline 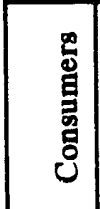 & 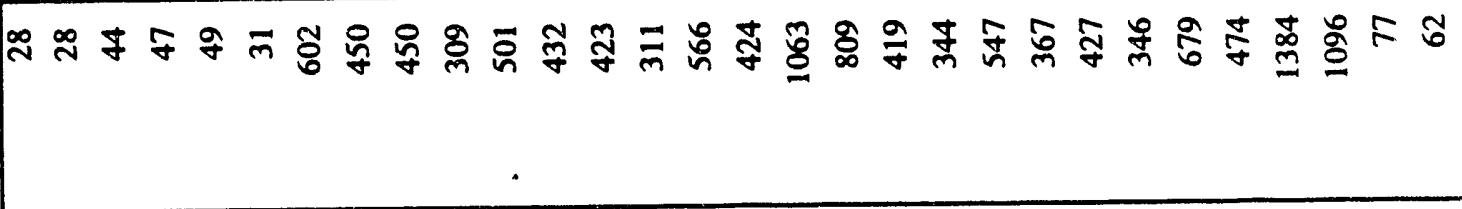 \\
\hline 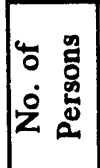 & 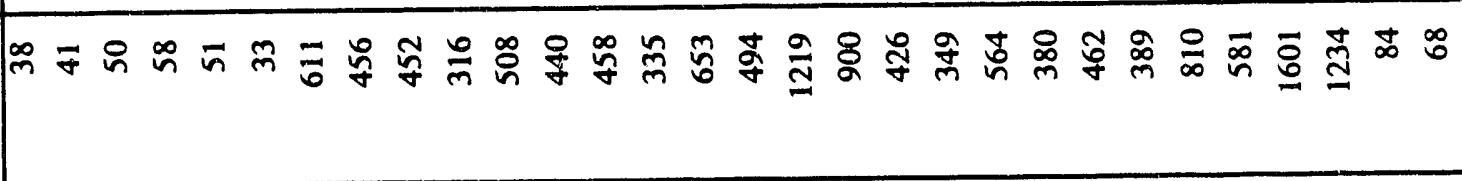 \\
\hline | & 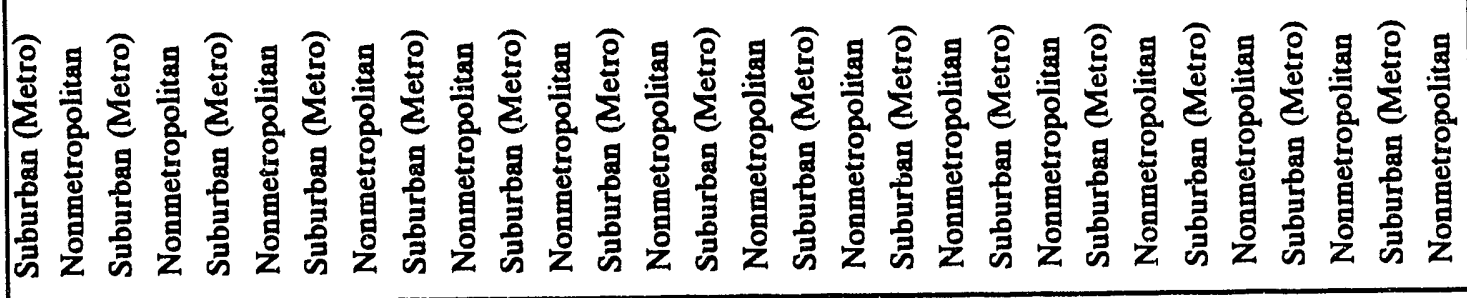 \\
\hline & 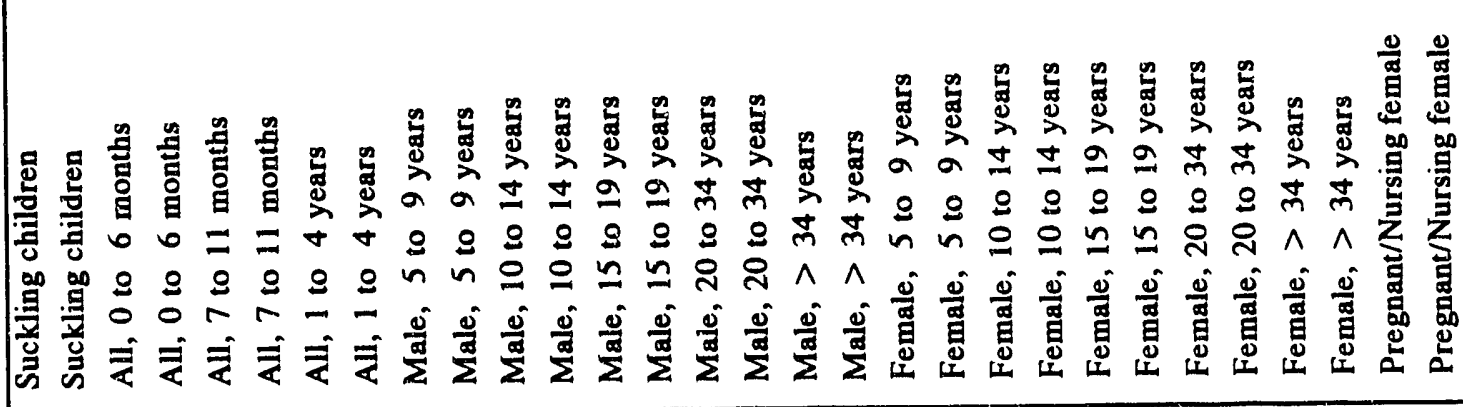 \\
\hline
\end{tabular}

C. 58 


\begin{tabular}{|c|c|}
\hline 票 & 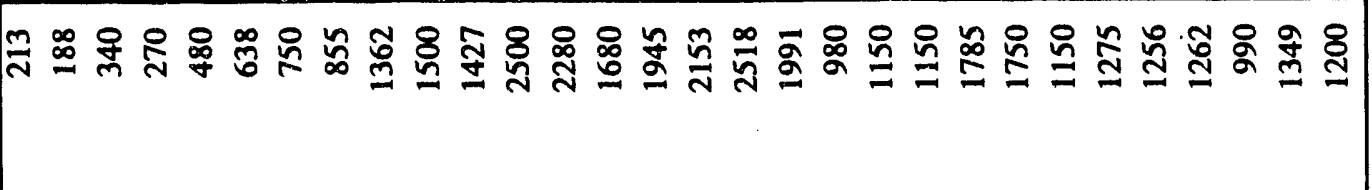 \\
\hline$\stackrel{8}{8}$ & 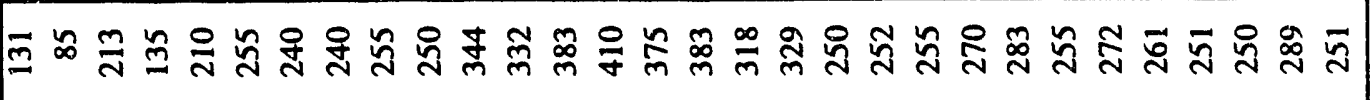 \\
\hline$\frac{1}{2}$ & ป็ \\
\hline se & 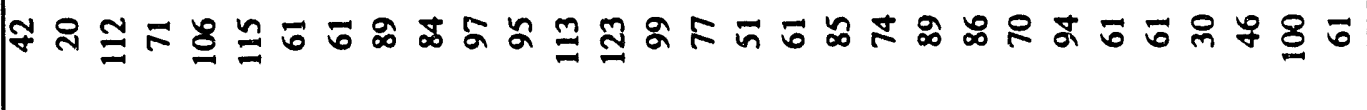 \\
\hline 睡 & In \\
\hline$\stackrel{0}{\mathrm{I}}$ & 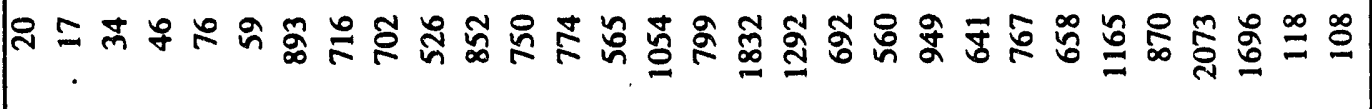 \\
\hline 兽 & 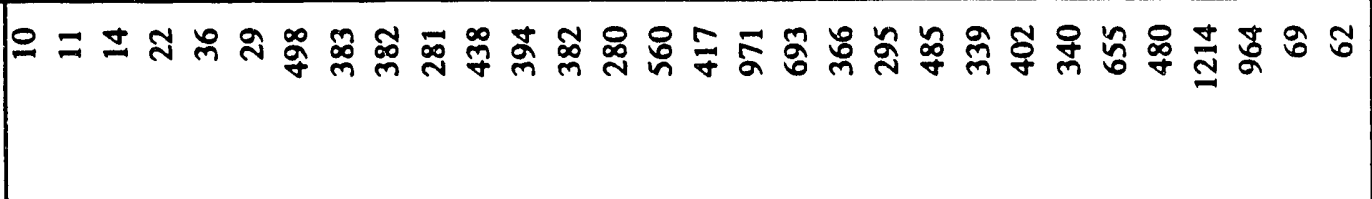 \\
\hline 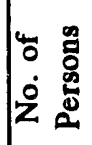 & 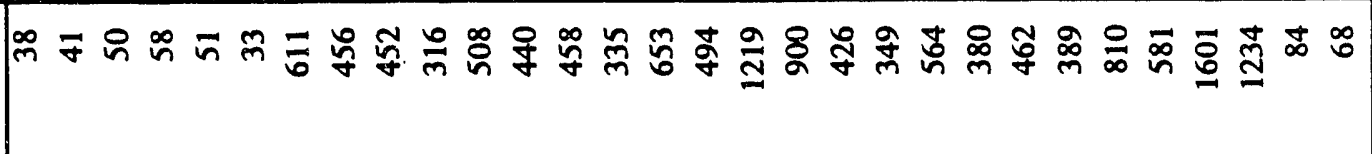 \\
\hline 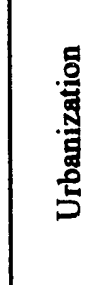 & 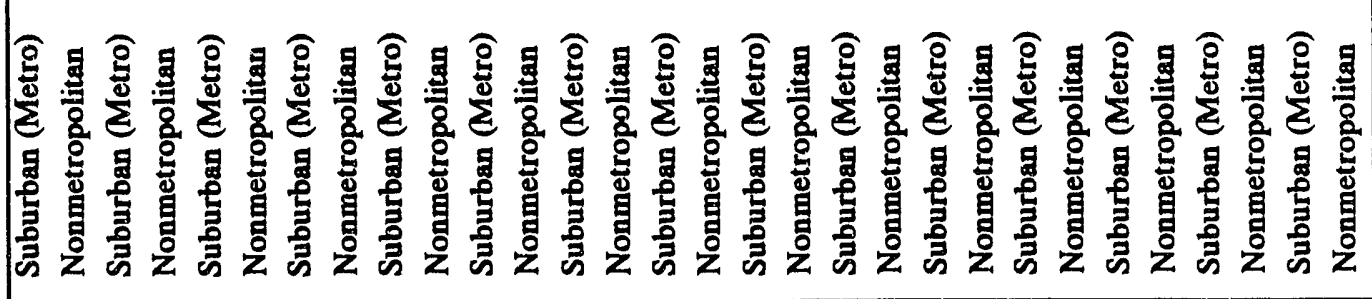 \\
\hline 赵 & 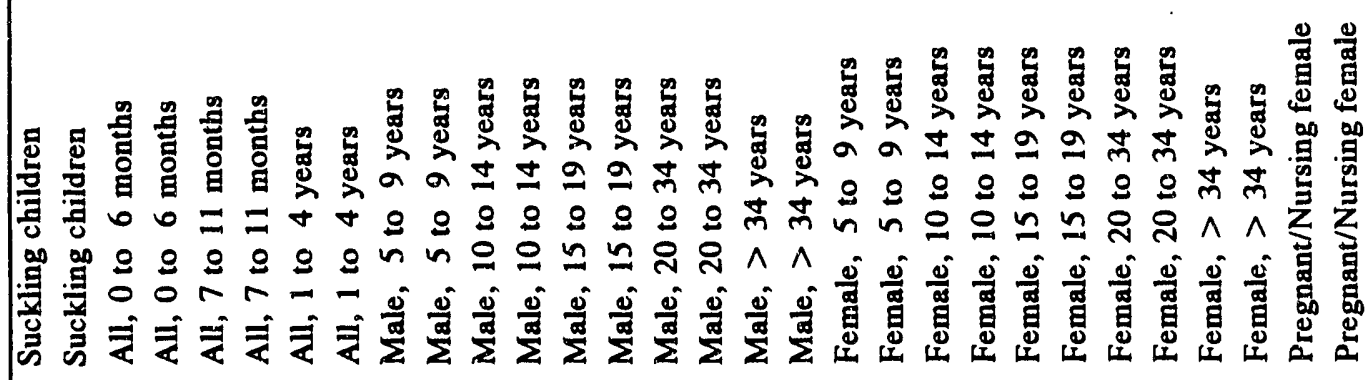 \\
\hline
\end{tabular}




\begin{tabular}{|c|c|}
\hline 悬 & 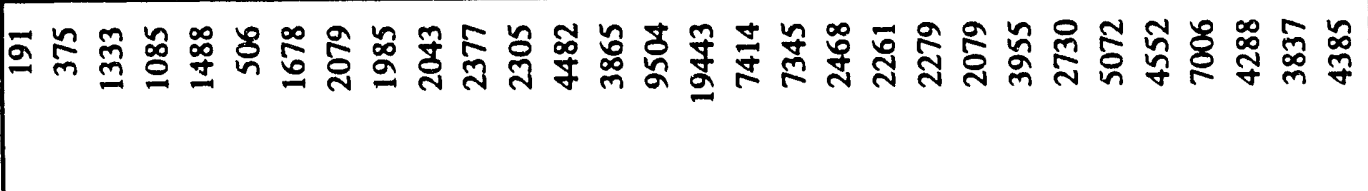 \\
\hline$\stackrel{n}{2}$ & 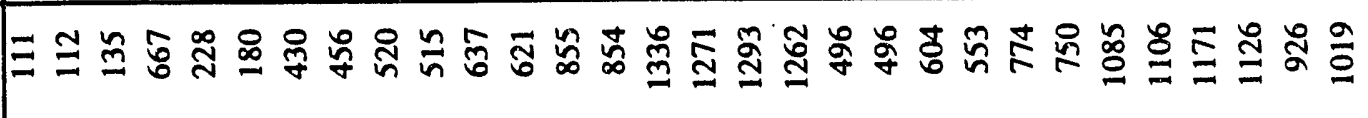 \\
\hline 恶 & 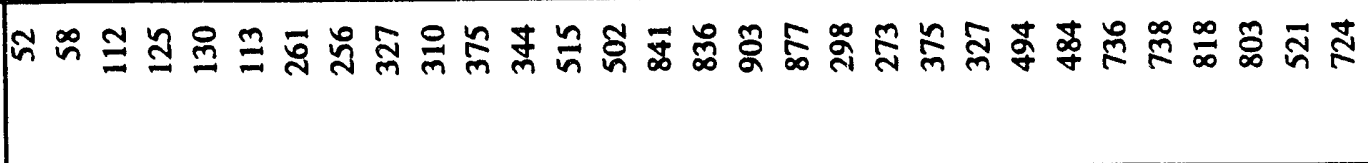 \\
\hline$g$ & 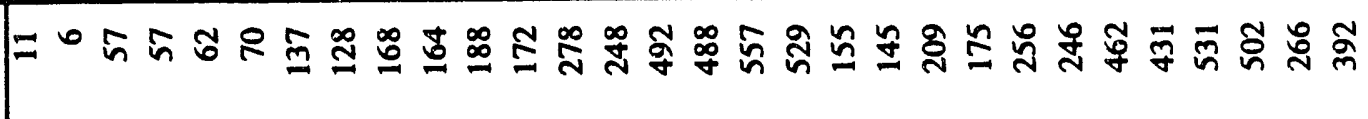 \\
\hline 量拿 & 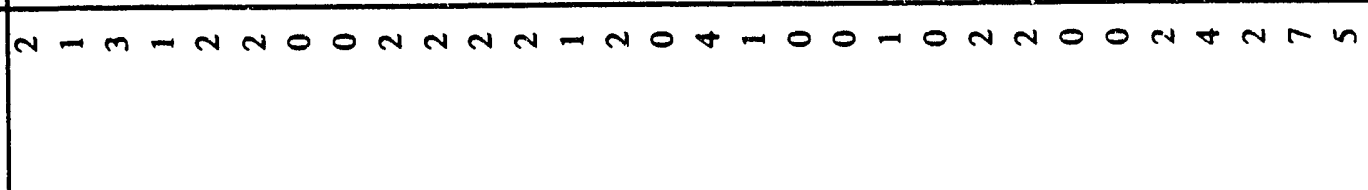 \\
\hline$\stackrel{n}{a}$ & 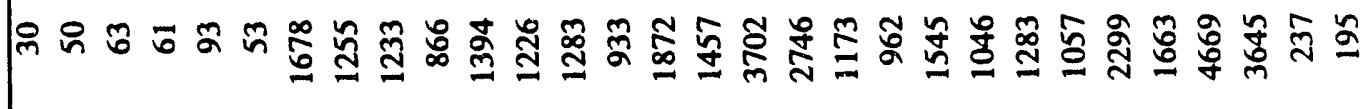 \\
\hline 要 & モ ล ล \\
\hline 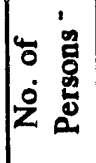 & 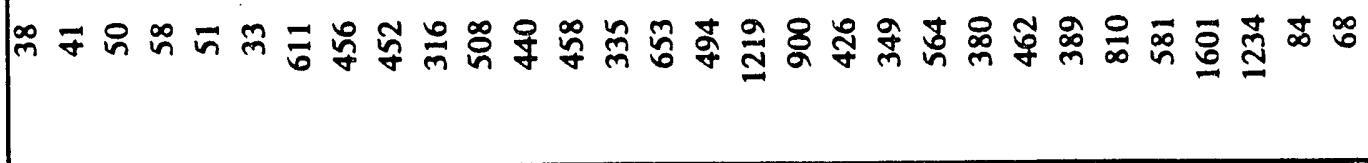 \\
\hline 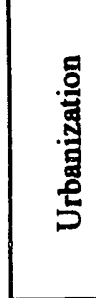 & 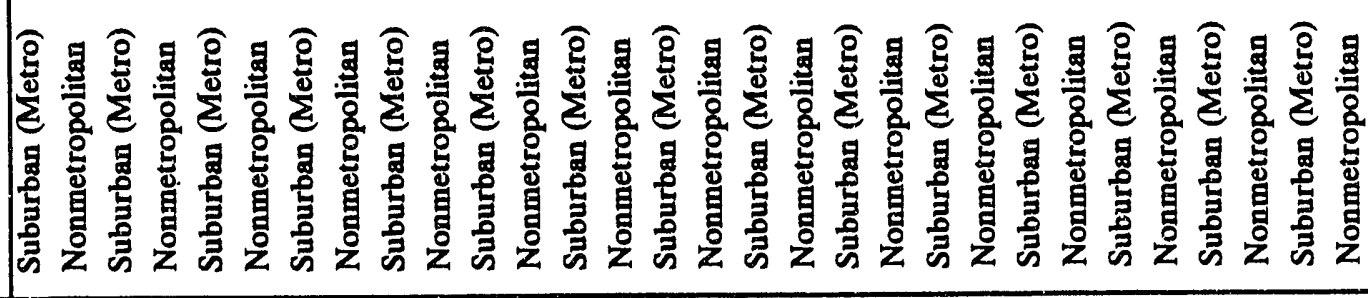 \\
\hline 裉 & 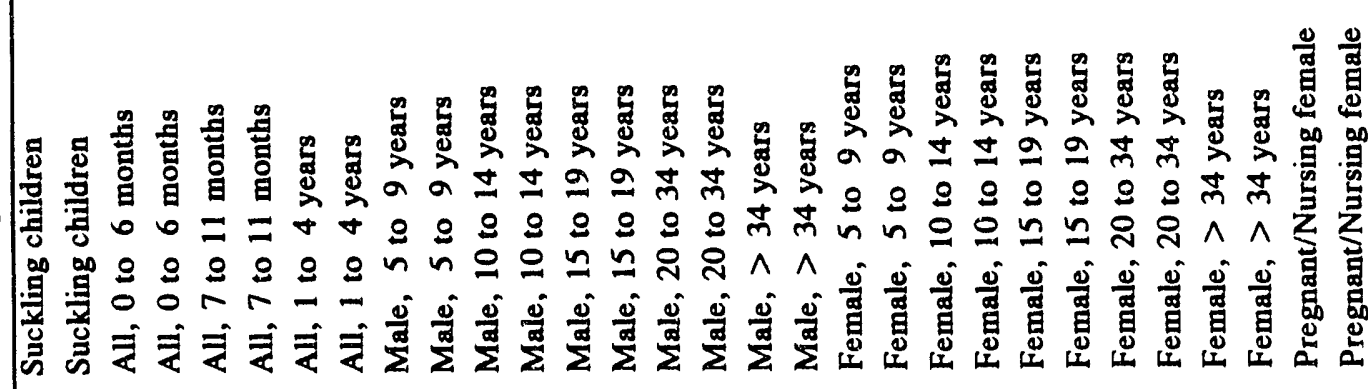 \\
\hline
\end{tabular}


Table C.60. Food Groups Corresponding to Column Headings in Tables C.61-C.63

\begin{tabular}{|c|c|c|c|}
\hline Column & Food & Column & Food \\
\hline 1 & Fresh milk and as ingredient & 33 & Fresh white potatoes \\
\hline 2 & Cream and as ingredient & 34 & Fresh sweet potatoes \\
\hline 3 & Cottage cheese & 35 & Processed potatoes \\
\hline 4 & Other cheese & 36 & Vegetable juices \\
\hline 5 & Ice cream and other frozen & 37 & Apples \\
\hline 6 & Baby formula and canned milk & 38 & Pears \\
\hline 7 & Dried milk products & 39 & Strawberries \\
\hline 8 & Goat milk & 40 & Other berries \\
\hline 9 & Fresh green peas & 41 & Sweet cherries \\
\hline 10 & Fresh corn & 42 & Melons \\
\hline 11 & Fresh asparagus & 43 & Grapes \\
\hline 12 & Fresh tomatoes & 44 & Apricots \\
\hline 13 & Fresh snap beans & 45 & Peaches \\
\hline 14 & Fresh cabbage & 46 & Plums/prunes \\
\hline 15 & Fresh lettuce & 47 & Other noncitrus fruits \\
\hline 16 & Fresh spinach & 48 & Noncitrus fruit juices \\
\hline 17 & Fresh cauliflower & 49 & Beef \\
\hline 18 & Fresh celery & 50 & Pork \\
\hline 19 & Fresh broccoli & 51 & Chicken \\
\hline 20 & Fresh carrots & 52 & Other poultry \\
\hline 21 & Fresh onions & 53 & Game \\
\hline 22 & Fresh beets & 54 & Cured pork \\
\hline 23 & Fresh turnips, rutabagas & 55 & Lunch meat \\
\hline 24 & Squash, pumpkin & 56 & Other fresh meat \\
\hline 25 & Fresh cucumbers & 57 & Eggs \\
\hline 26 & Fresh green peppers & 58 & Local fish \\
\hline 27 & Fresh lima beans & 59 & Other fish \\
\hline 28 & Other fresh leafy vegetables & 60 & Shellfish \\
\hline 29 & Other fresh vegetables & 61 & Bread and rolls \\
\hline 30 & Tomatoes, canned/frozen & 62 & Other bakery products \\
\hline 31 & Stored vegetables & 63 & Food mixtures \\
\hline 32 & Vegetable mixes, soups & 64 & Other food \\
\hline
\end{tabular}




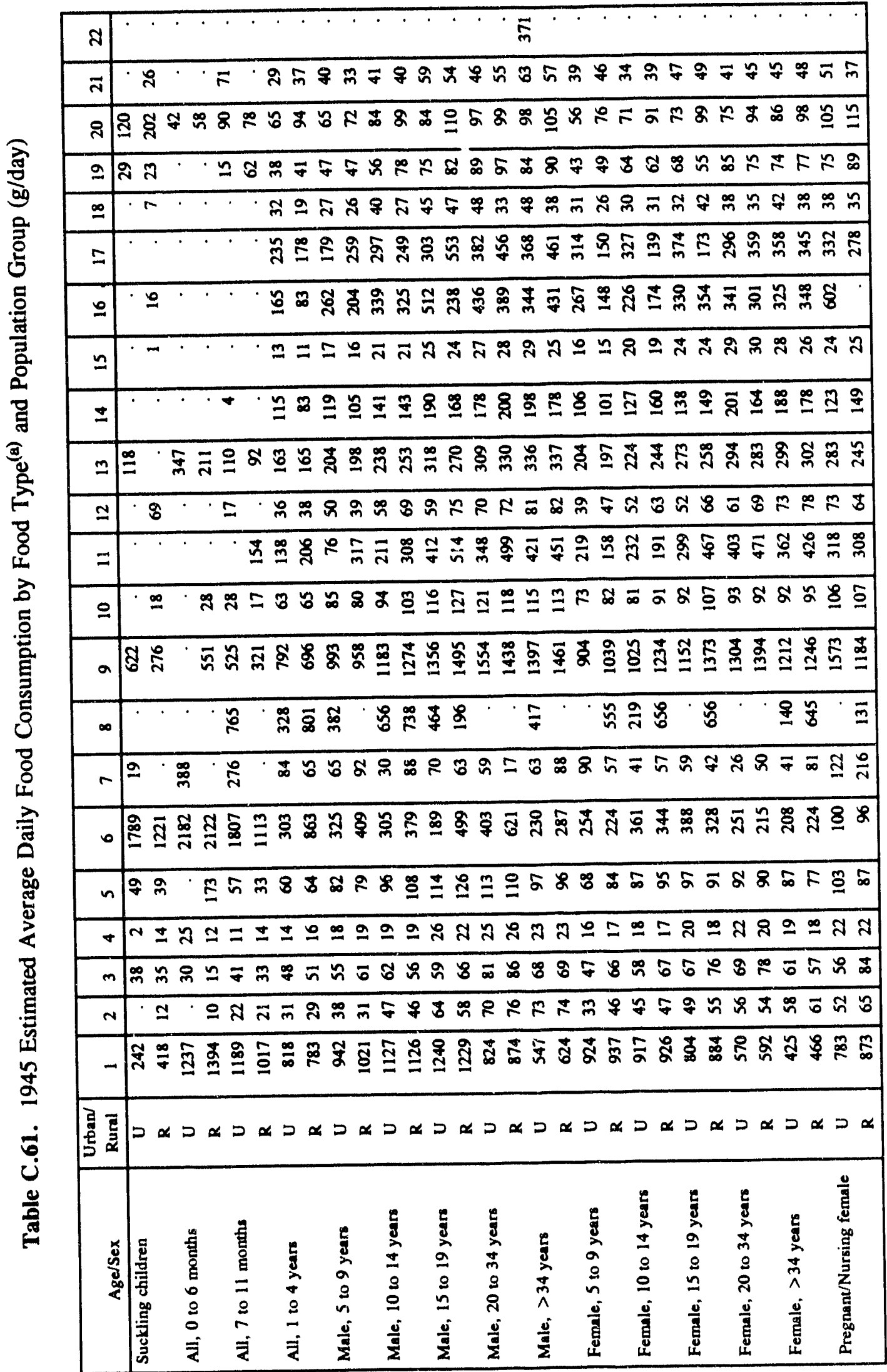

C. 62 


\begin{tabular}{|c|c|}
\hline$\%$ & 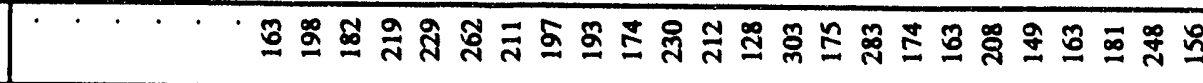 \\
\hline 7 & 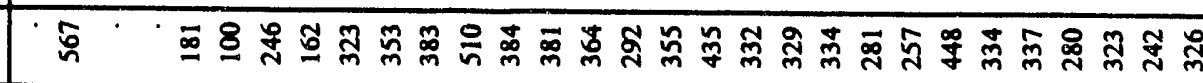 \\
\hline$F$ & 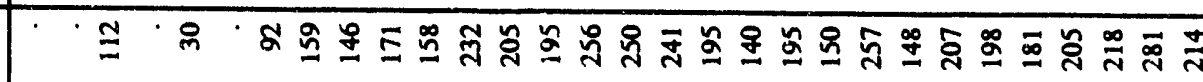 \\
\hline 9 & 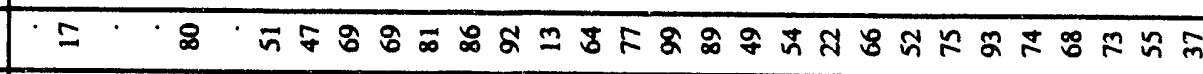 \\
\hline is & 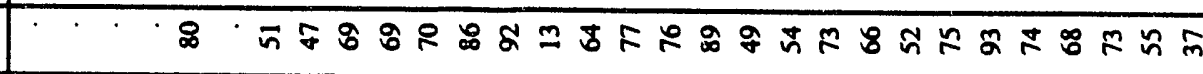 \\
\hline \begin{tabular}{c|c}
$\infty$ \\
$\infty$
\end{tabular} & สุ๊ \\
\hline$\tilde{m}$ & 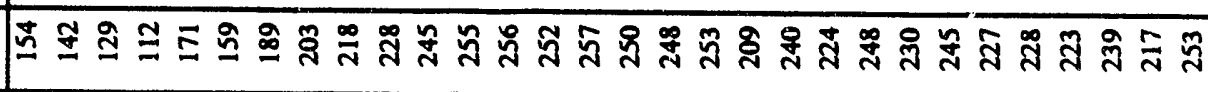 \\
\hline$\stackrel{0}{2}$ & 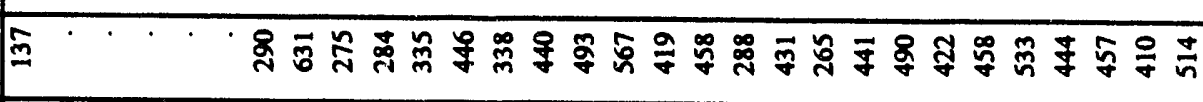 \\
\hline$\approx$ & 00000000000000000000000000 \\
\hline$\overline{\mathrm{m}}$ & 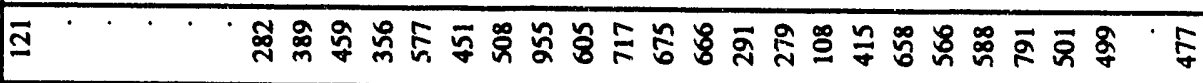 \\
\hline$m \approx$ & 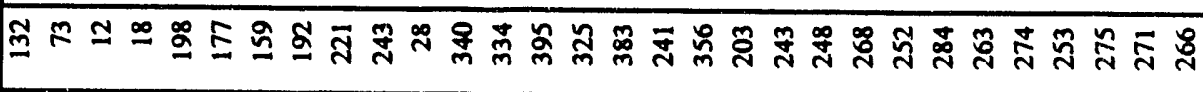 \\
\hline$\approx$ & 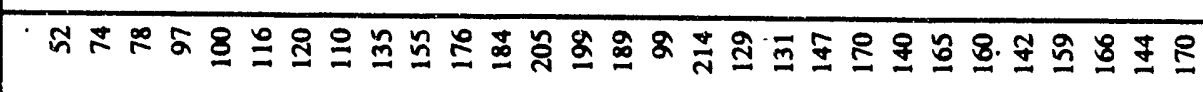 \\
\hline $\bar{m}=$ & 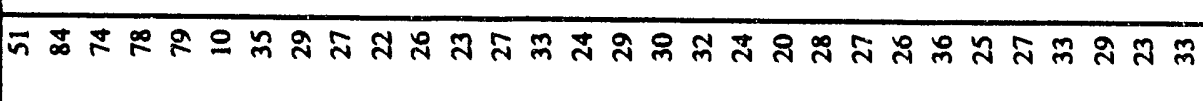 \\
\hline क्लीज & 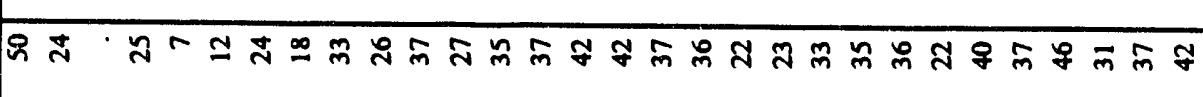 \\
\hline i) & 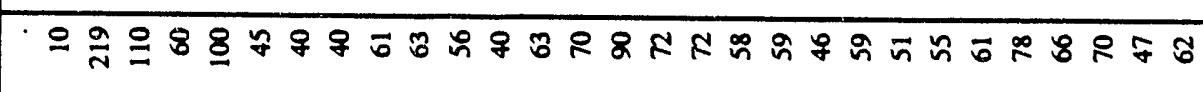 \\
\hline 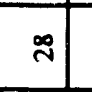 & 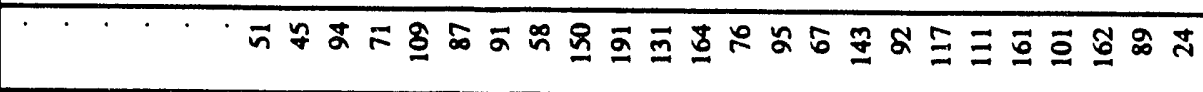 \\
\hline ה & 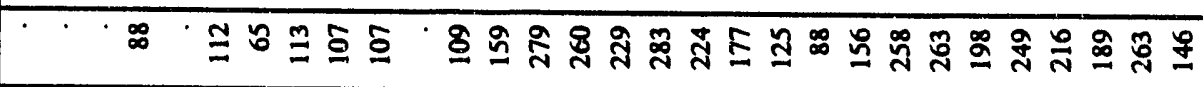 \\
\hline if & 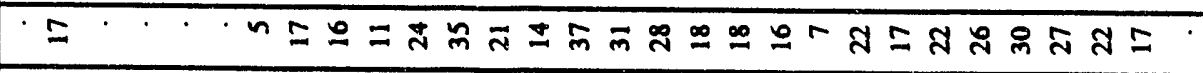 \\
\hline a & 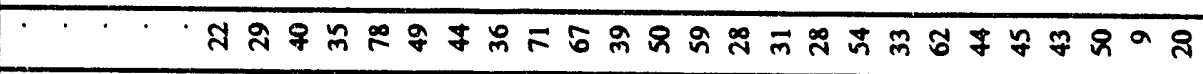 \\
\hline 48 & 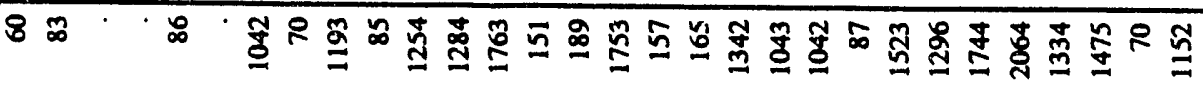 \\
\hline ก) & 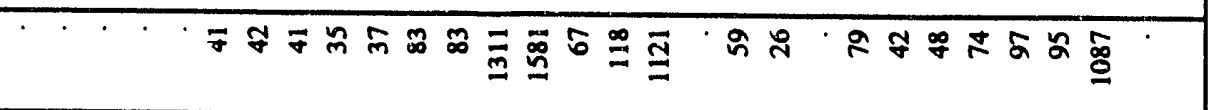 \\
\hline$=$ & 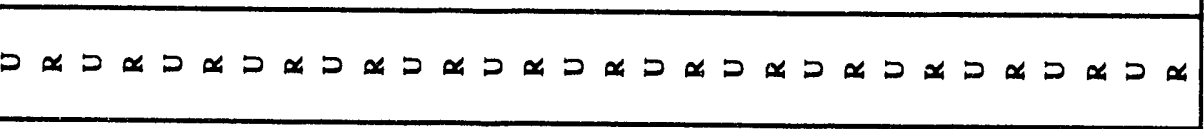 \\
\hline $\begin{array}{ll}0 \\
0 \\
0\end{array}$ & $\begin{array}{lllllllllllllll} & & & & & & & & & & & \\
0\end{array}$ \\
\hline
\end{tabular}




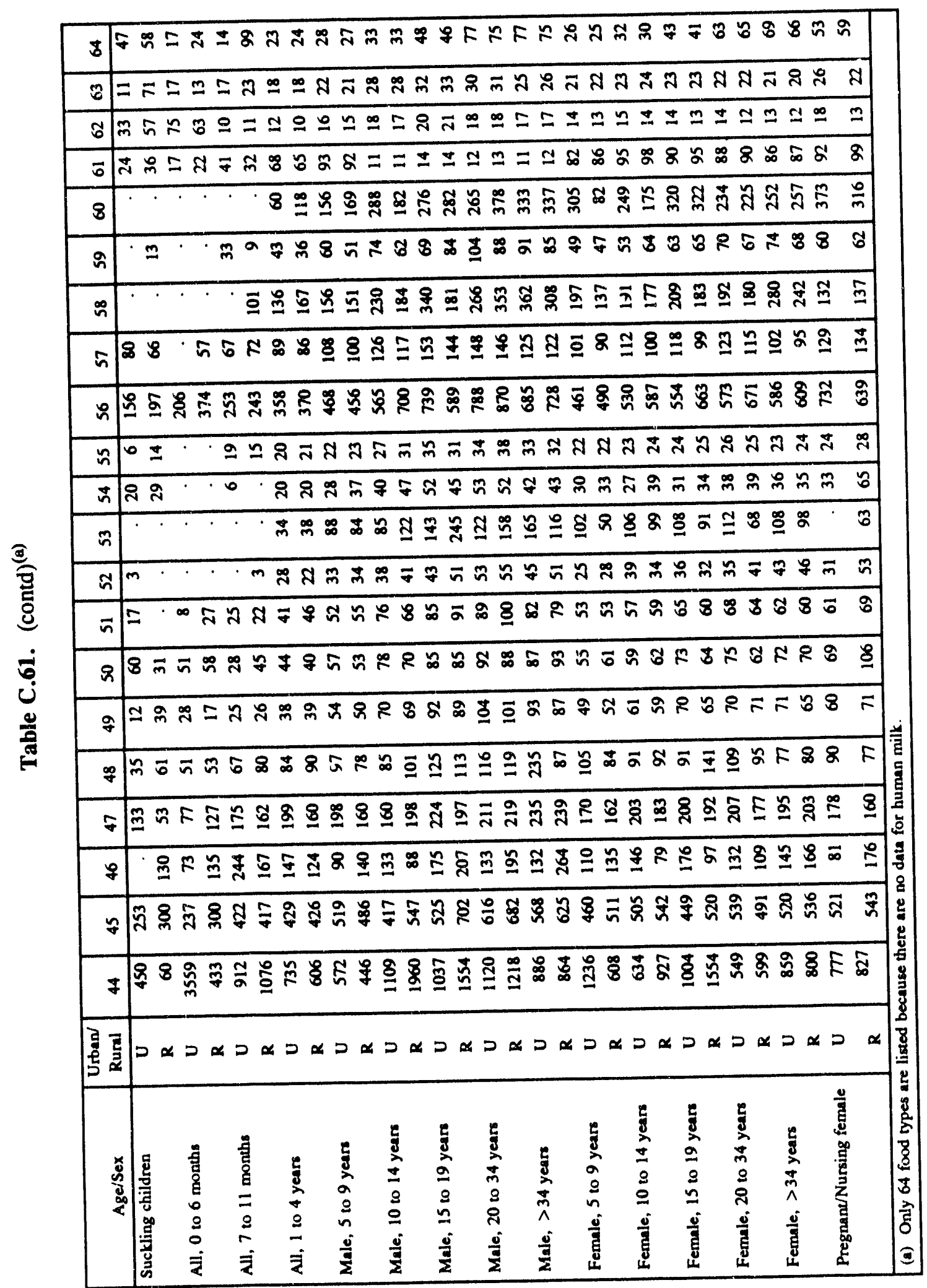




\begin{tabular}{|c|c|}
\hline$\pi$ & ฉి \\
\hline$\overline{\mathbf{N}}$ & 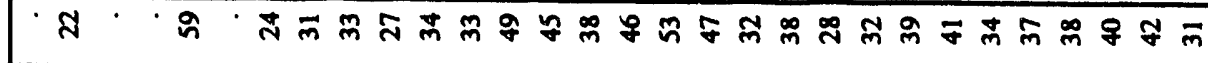 \\
\hline त) & 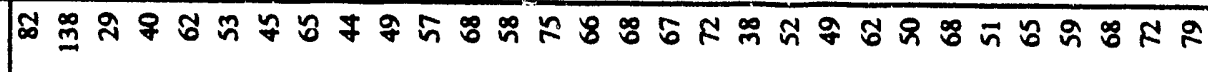 \\
\hline a & 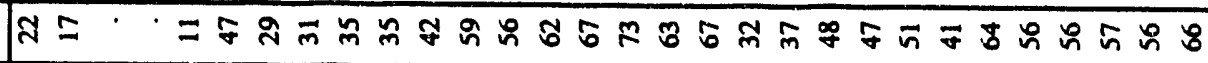 \\
\hline$\stackrel{-\infty}{-\infty}$ & 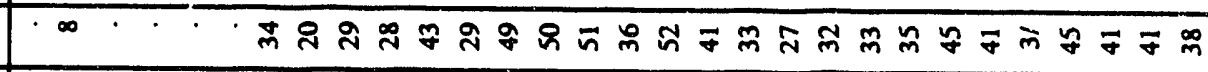 \\
\hline$=$ & 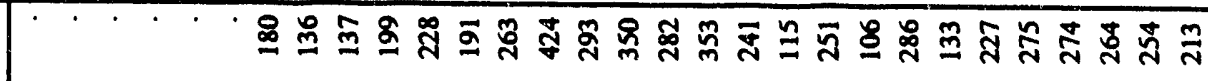 \\
\hline - & 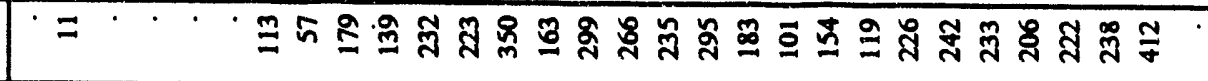 \\
\hline$\simeq$ & 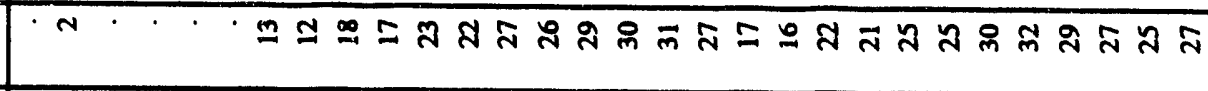 \\
\hline \pm & 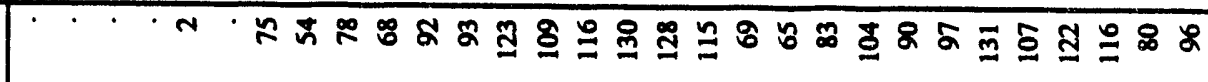 \\
\hline m & 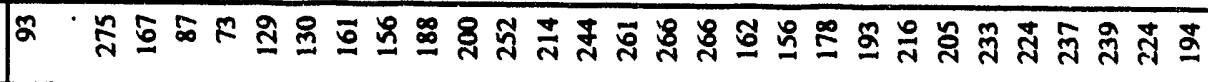 \\
\hline$\cong$ & 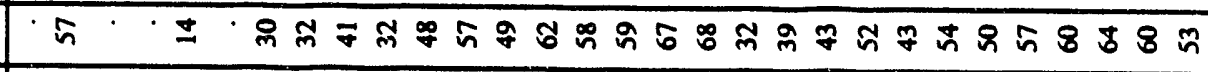 \\
\hline$=$ & 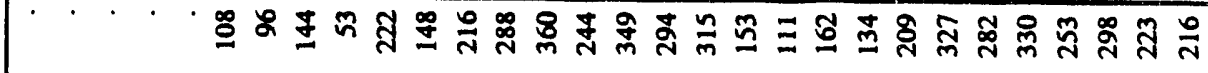 \\
\hline 요 & 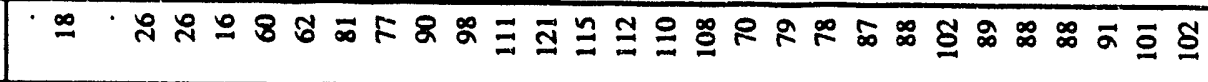 \\
\hline$a$ & 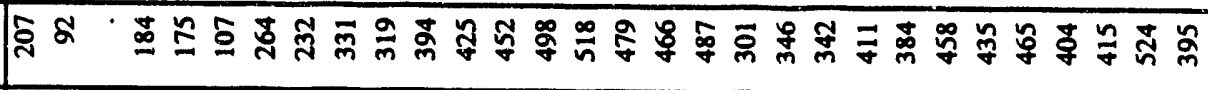 \\
\hline$\infty$ & 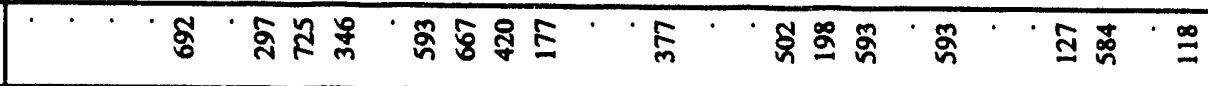 \\
\hline - & 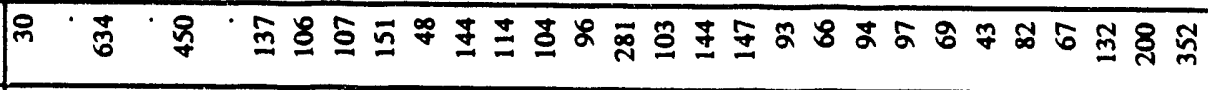 \\
\hline 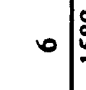 & 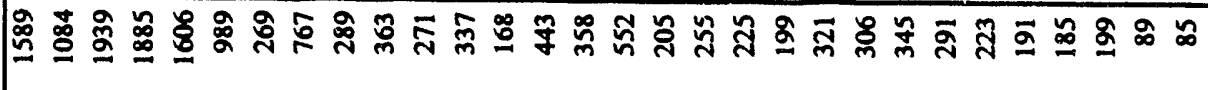 \\
\hline$n=$ & 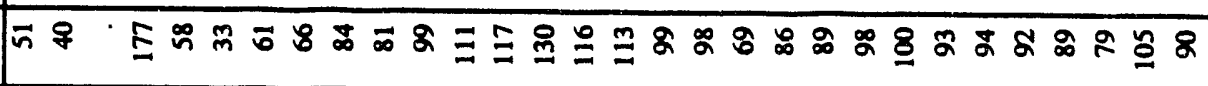 \\
\hline+ & 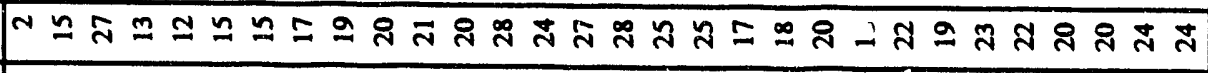 \\
\hline$m 5$ & 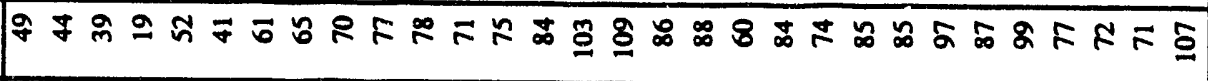 \\
\hline N & 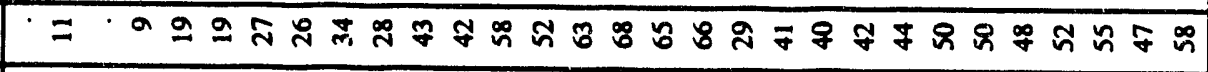 \\
\hline$-\%$ & 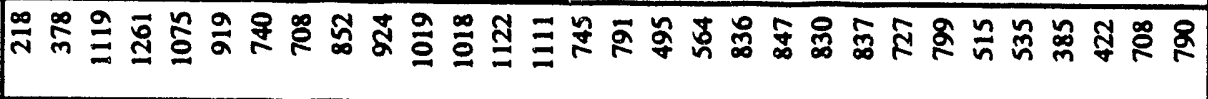 \\
\hline : & $\partial \propto \partial \propto \partial \propto \partial \alpha \supset \alpha \supset \propto$ \\
\hline 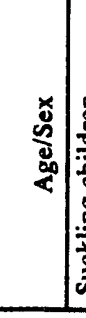 & 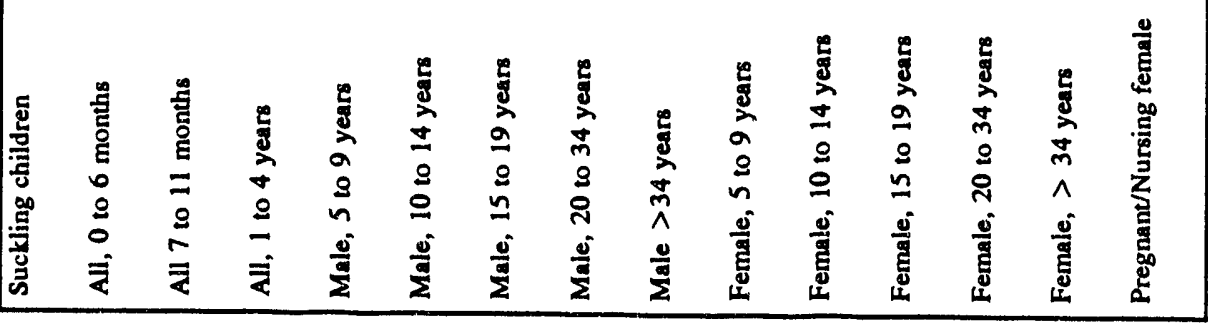 \\
\hline
\end{tabular}

C. 65 


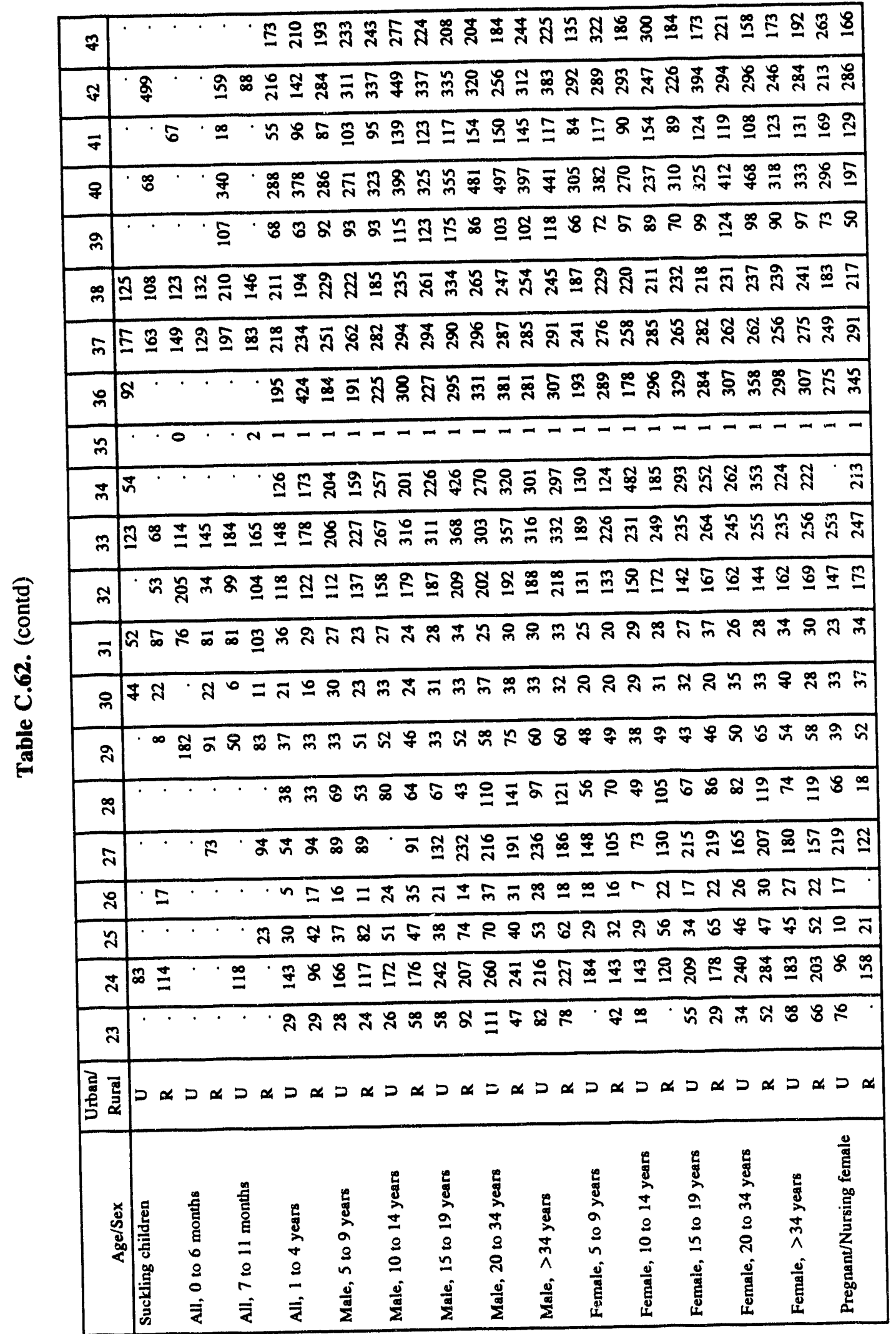




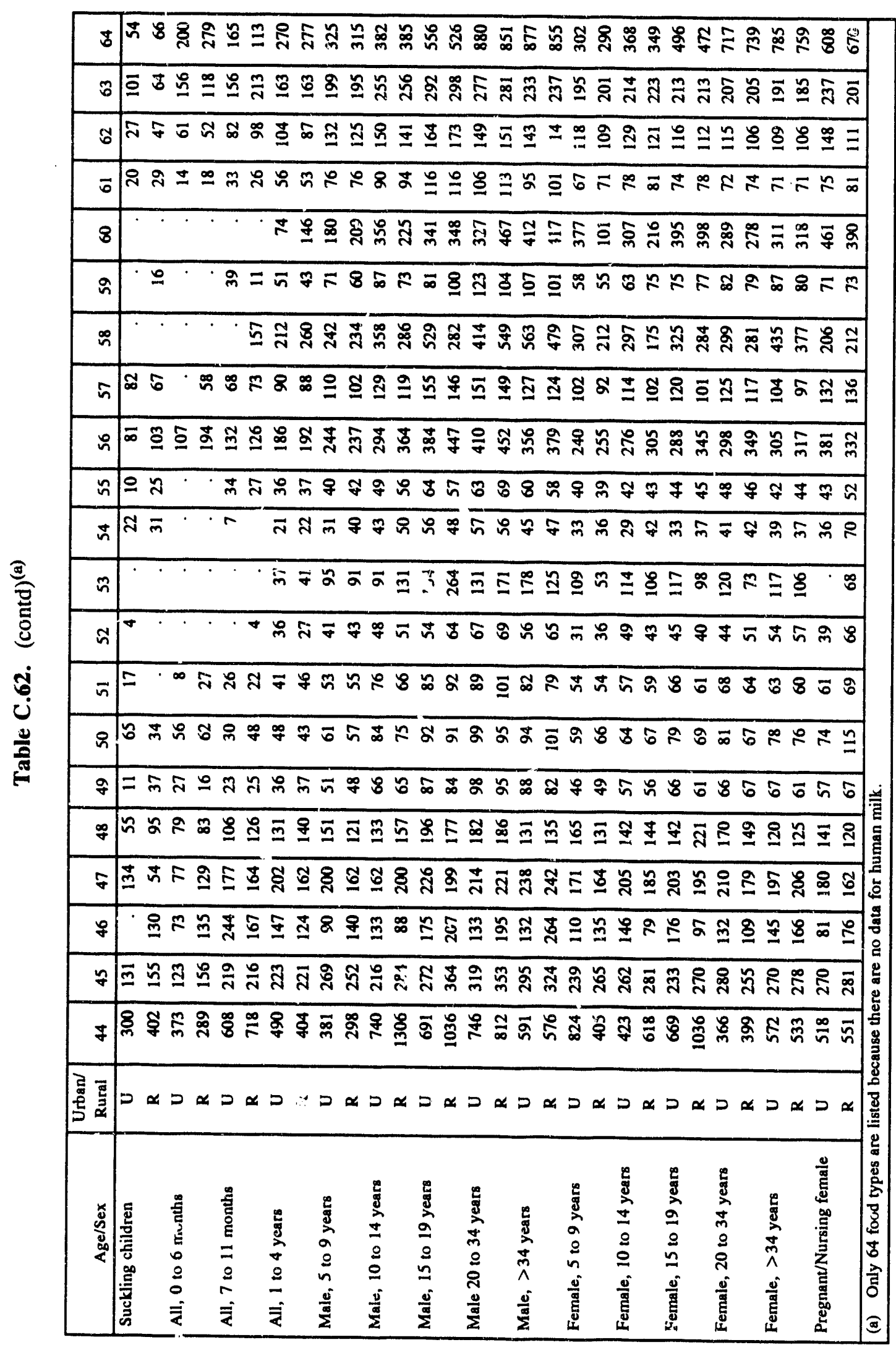




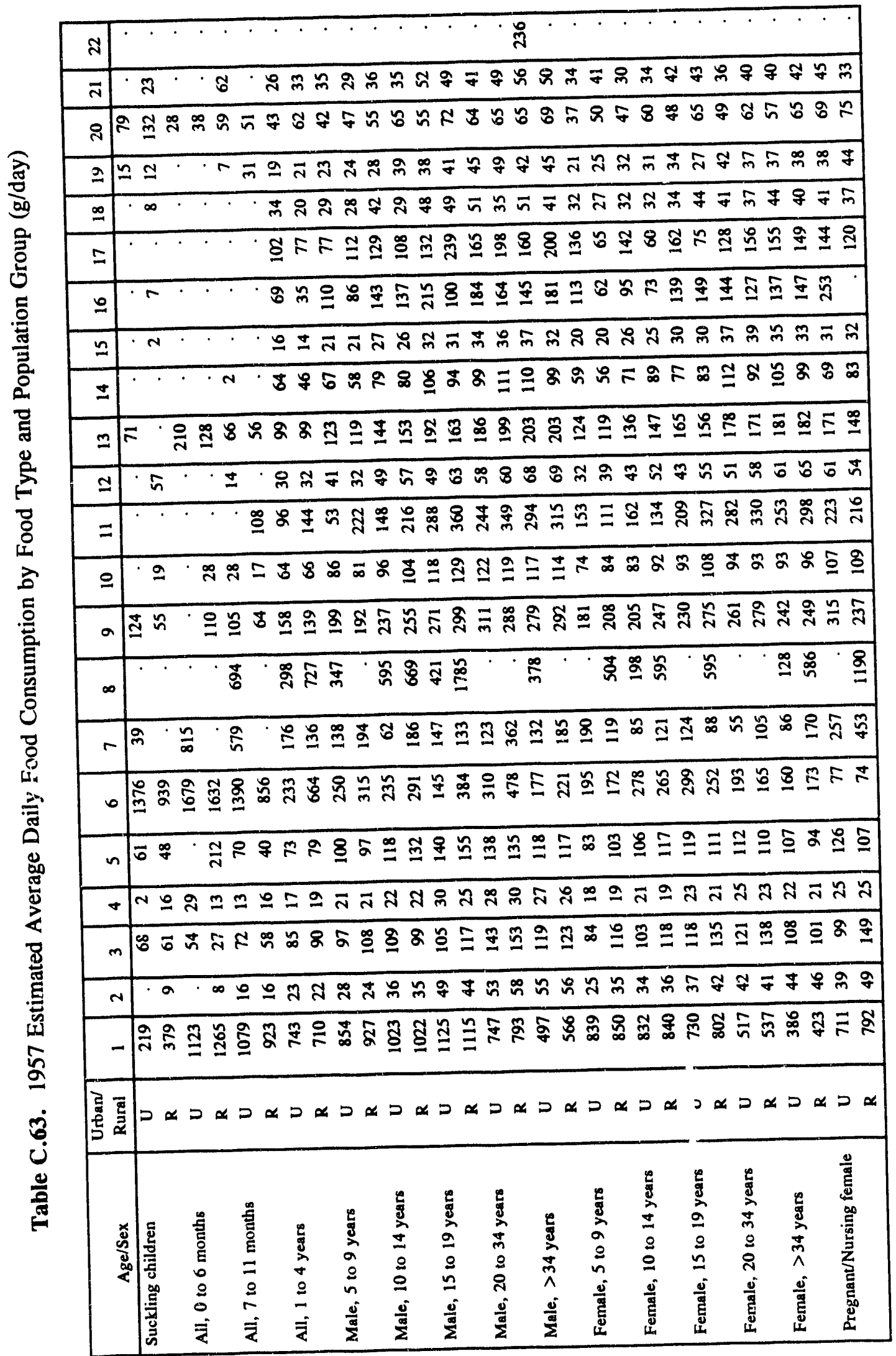




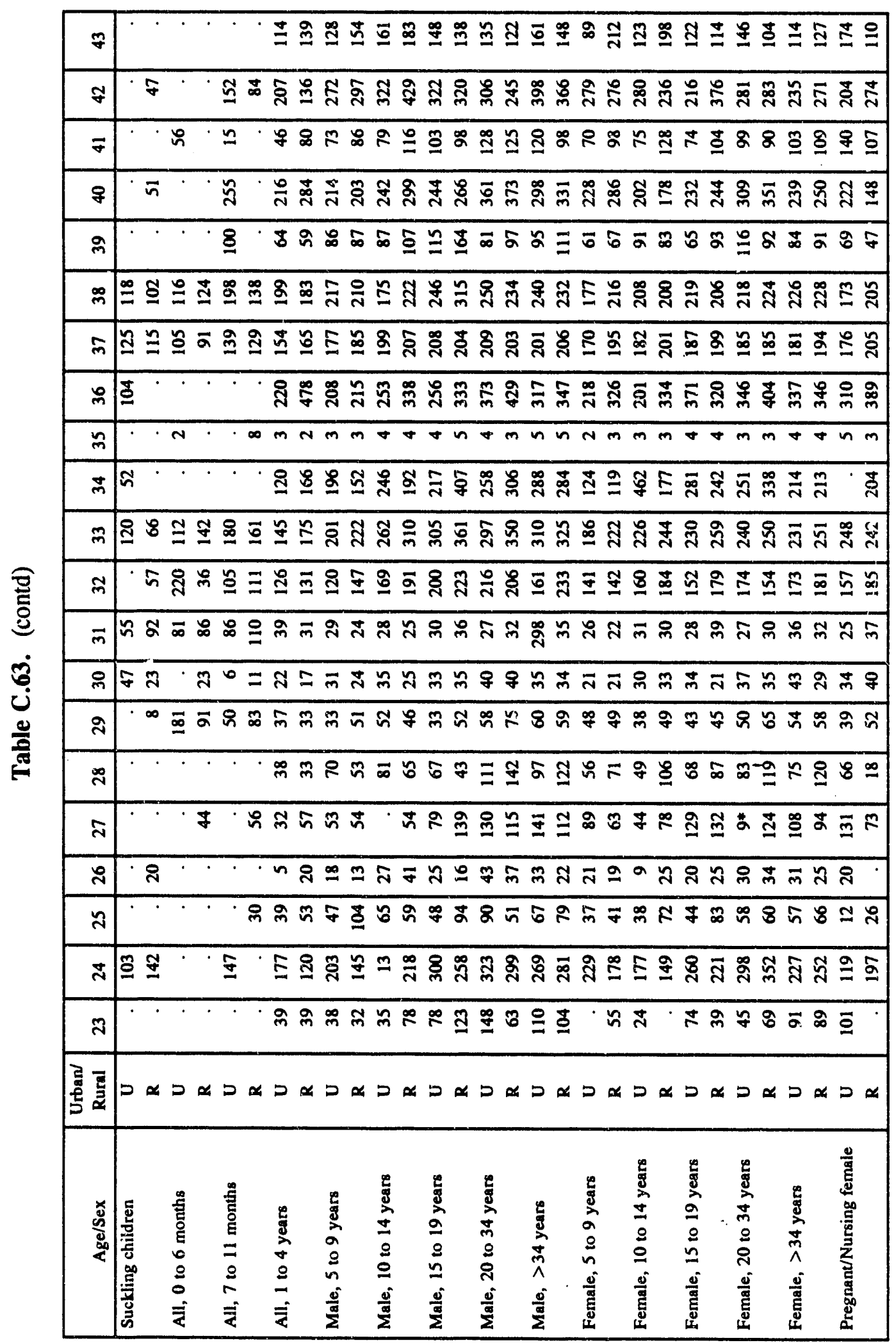




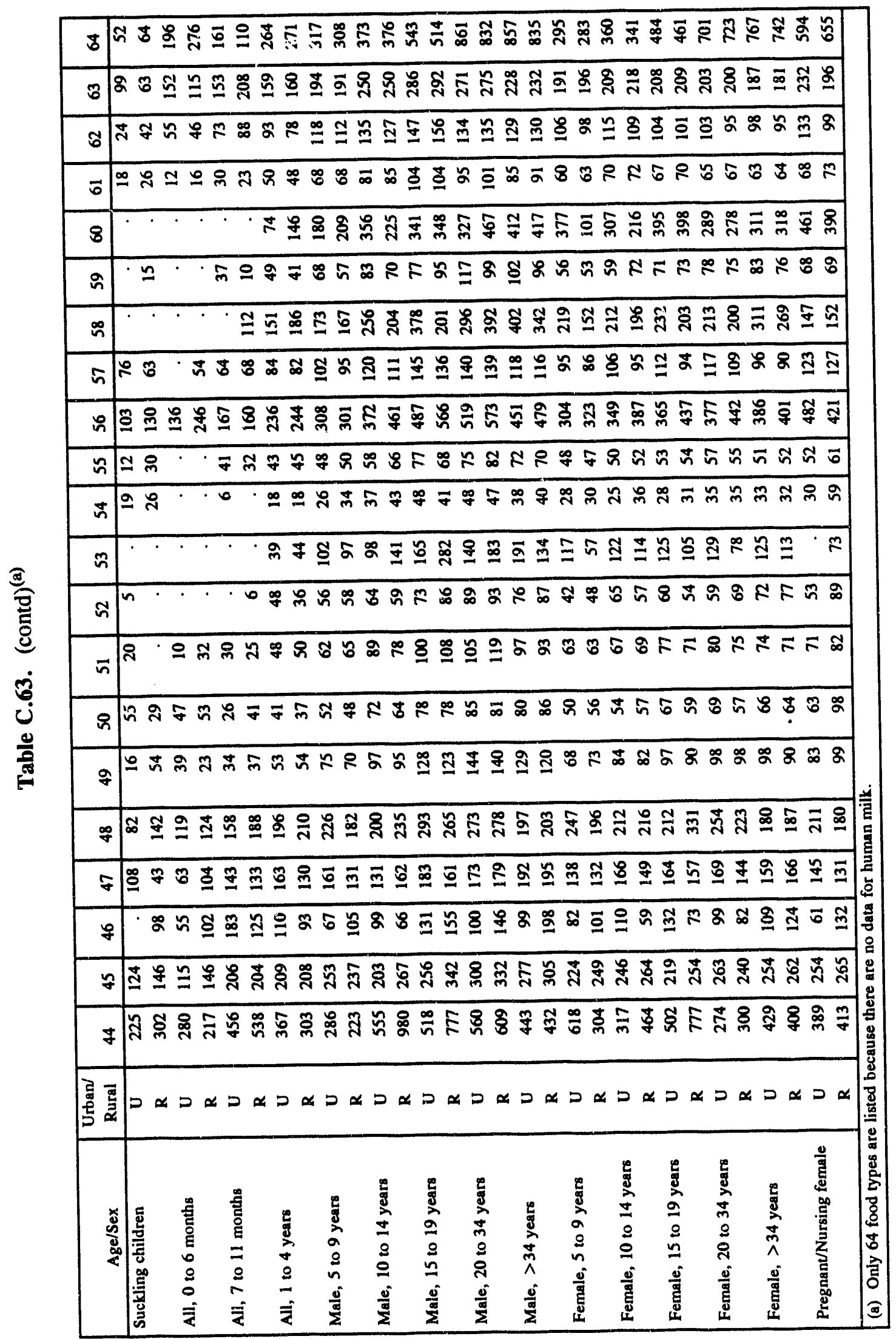

C. 70 


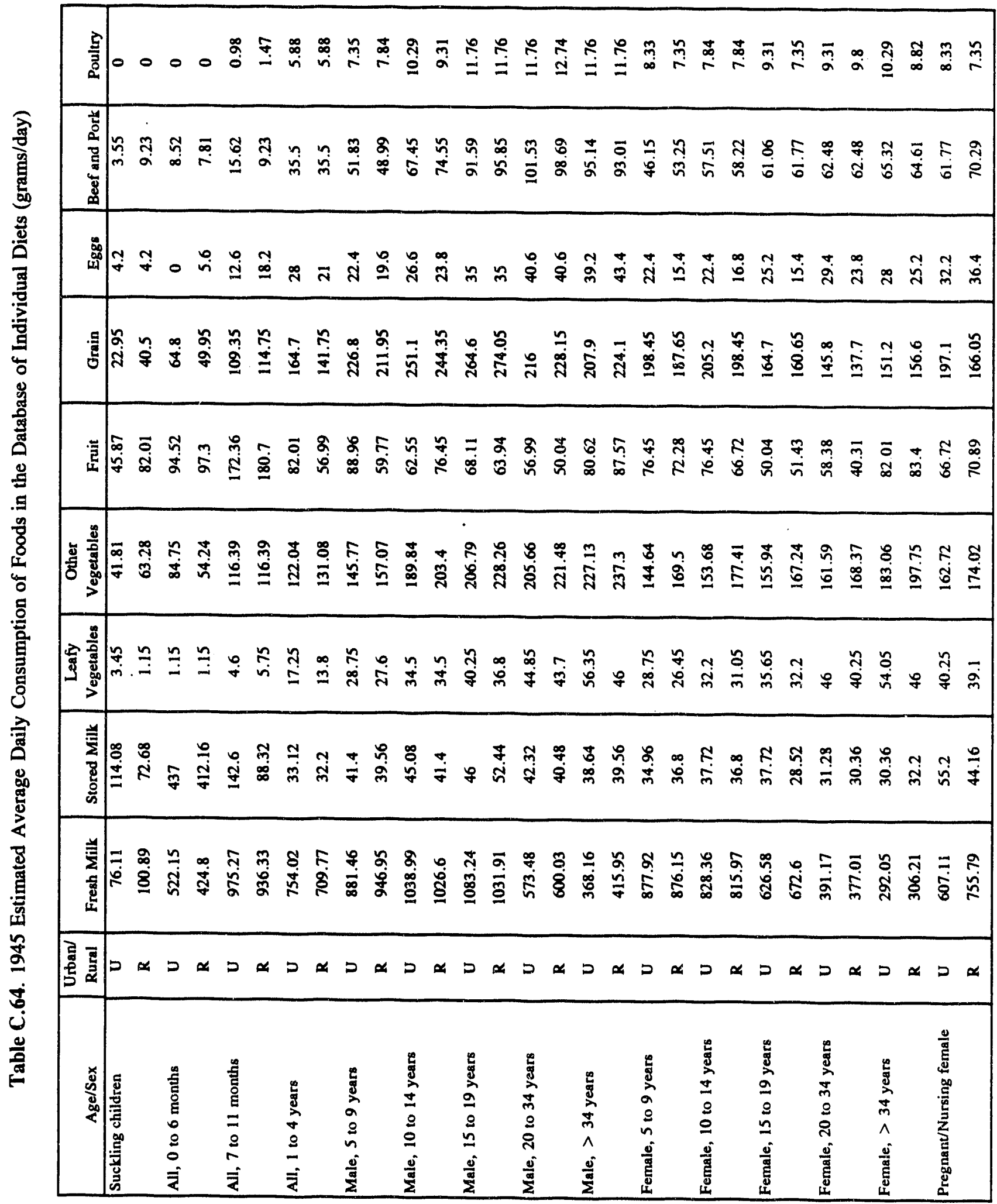

C. 71 


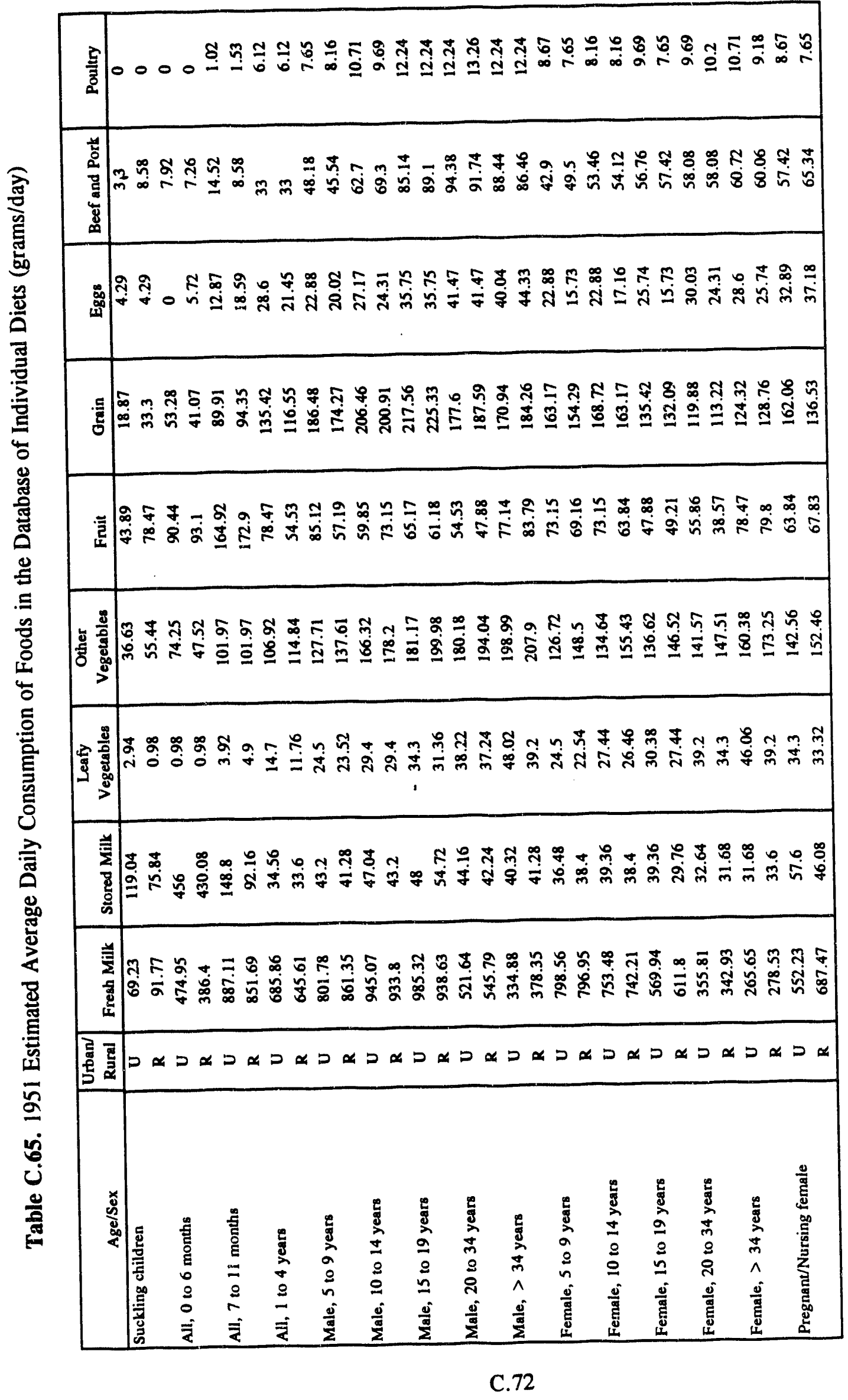




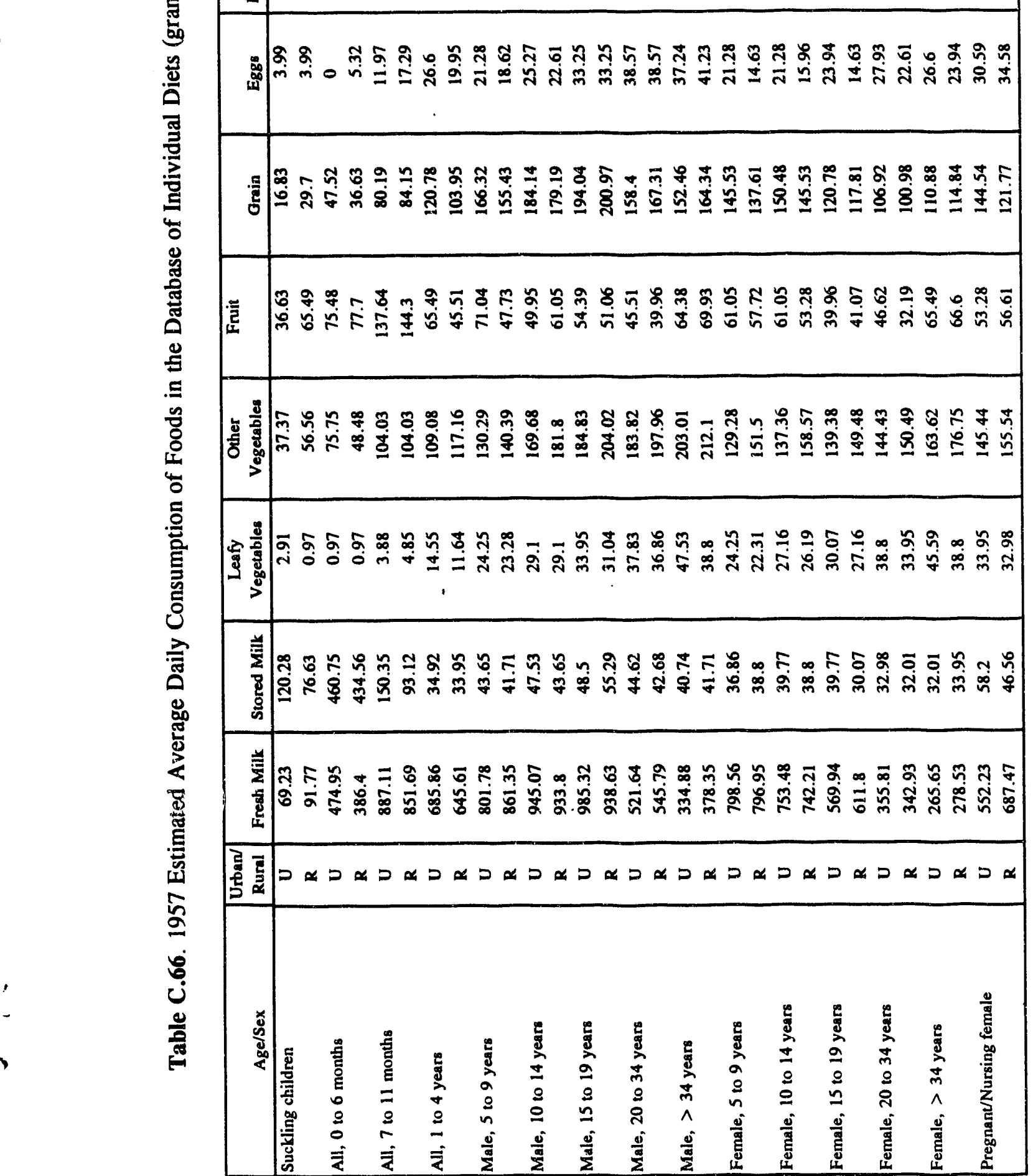

C. 73 


\section{DISTRIBUTION}

No. of

Copies

OFFSITE

Technical Steering Panel

D. S. Barth

University of Nevada

4505 Maryland Parkway

Las Vegas, NV 89154

W. A. Bishop

2503 Wedgewood Court S.E.

01 ympia, WA 98501

M. L. Blazek

Oregon Department of Energy

625 Marion Street N.E.

Salem, OR 97310

G. G. Caldwell

Tulsa City-County Health Dept.

4616 East 15th Street

Tulsa, OK 74112

S. N. Davis

Dept. of Hydrology and Water Resources

Building 11

University of Arizona

Tucson, AZ 85721

N. J. Germond

224 Iron Mountain Blvd.

Lake Oswego, OR 97034

P. C. K1 ingeman

Civil Engineering Dept.

Appejin Hall 202

Oregon State University

Corvallis, OR 97331-2302

K. J. Kopecky

Fred Hutchinson Cancer

Research Center

1124 Columbia Street

Seattle, WA 98104
No. of

Copies

P. D. McGavran

Dept. of Health and Welfare

450 W. State Street, 4th Floor Boise, ID 83720-5450

R. L. Morrill

Dept. of Geography, DP-10

University of Washington

Seattle, WA 98195

A. H. Murphy

3115 NW McKinley Drive

Corvallis, OR 97330

D. W. Price

Agricultural Economics

Hulbert Hall Room 211

Washington State University

Pullman, WA 99164-6210

M. A. Robkin

Radiological Sciences, SB-75

University of Washington

Seattle, WA 98195

G. S. Roessler

Route 1, Box $139 \mathrm{H}$

Elysian, MN 56028

B. Shleien

2421 Homestead Drive

Silver Springs, MD 20902

A. P. Slickpoo, Sr. 809 Nez Perce Lane

P.0. Box 331

Kamiah, ID 83536

J. E. Til1

Route 2 Box 122

Neeses, SC 29107 
No. of

Copies

D. E. Walker, Jr.

P.0. Box 4147

Boulder, CO 80306

Other

2 DOE Office of Scientific and Technical Information Technical Information Center P.0. Box 62

Oak Ridge, TN 37830

B. G. Brooks, EH-421

Room J-112

Department of Energy

Germantown, MD 20545

18 K. Charlee

Office of Nuclear Waste Mgmt.

Department of Ecology

719 Sleater Kinney Road, S.E.

Suite 2.00

Olympia, WA 98504

M. R. Donnelly

Public Health Service

Centers for Disease Control

2201 Sixth Avenue, RX-22

Seattle, WA 98121

R. S. Haber

1840 Willamette Street

Eugene, OR 97401

H. A. Haerer

Golden Associates, Inc.

4104 148th N.E.

Redmond, WA 98052

L. E. Sewell

Centers for Disease Control

4770 Buford Highway N.E., F-35

Atlanta, GA 30341-3724
No. of

Copies

J. P. Thomas

HEAL

1720 N. Ash

Spokane, WA 99205

\section{ONSITE}

6 DOE Richland Field Office

R. F. Brich, TSD

A5- 55

Public Reading Room (5)

A $1-65$

52 Battelle, Pacific Northwest Laboratories

D. M. Anderson (5)

K6-57

D. J. Bates

S. D. Cannon

K7 -34

J. M. Daer (5)

$\mathrm{K} 1-25$

D. H. Denham

$\mathrm{K} 1-25$

D. E. Deonigi

$\mathrm{K} 3-56$

W. T. Farris

$K 6-54$

M. D. Freshley

K3-54

R. 0. Gilbert

W. A. Glass (14)

$K 6-77$

K $7-34$

K4-13

R. H. Gray

S. P. Gydesen

$\mathrm{K} 1-33$

G. L. Harvey

P8-55

C. M. Heeb

$\mathrm{K} 1-77$

T. A. Ikenberry

K6 -42

K3-54

T. L. Marsh

K6-57

B. A. Napier

K3-54

S. J. Ouderkirk

K7 -34

J. V. Ramsdel1

K $6-03$

J. A. Shaw

$\mathrm{K} 1-25$

D. B. Shipler

$\mathrm{K} 1-25$

J. C. Simpson

K7-34

C. D. Taylor

B1 -40

W. L. Templeton

KI -30

M. E. Thiede

K6-13

W. H. Walters

K6-09

Publishing Coordination

Records Center (2)

$\mathrm{K} 1-06$

K3-70

Technical Library (2)

P8-55 

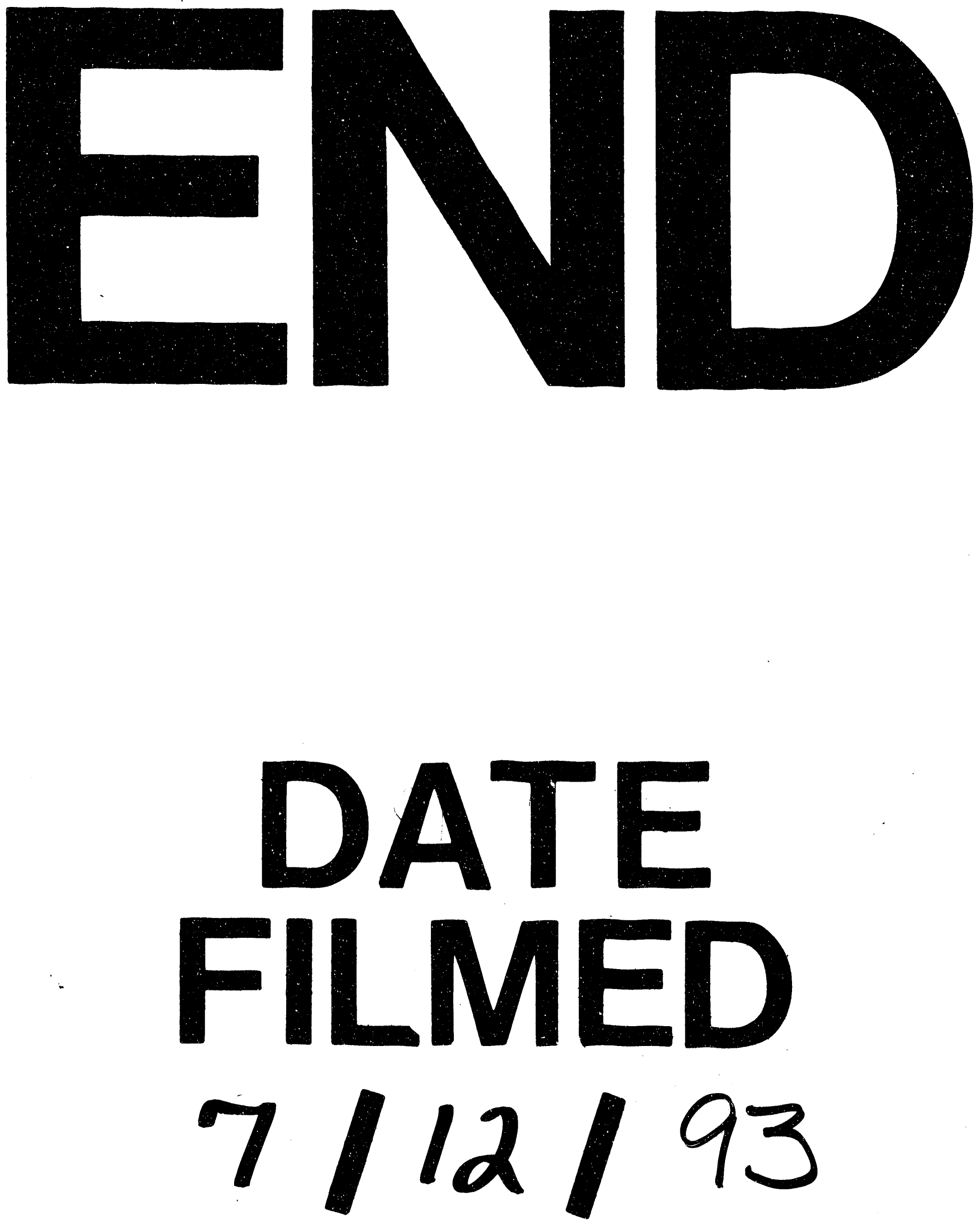

7 
